HU-P-D203

\title{
Observation of Central Exclusive Diphoton Production at the Tevatron
}

\author{
Jens Erik Brücken
}

Division of Elementary Particle Physics

Department of Physics

Faculty of Science

University of Helsinki

and

Helsinki Institute of Physics

Helsinki, Finland

\section{ACADEmic Dissertation}

To be presented for public criticism, with the permission of the Faculty of Science of the University of Helsinki, in the auditorium D101 of the Physicum building, Gustaf Hällströmin katu 2, on Friday, May $24^{\text {th }}, 2013$, at 14.15 o'clock.

Helsinki 2013 


\section{Supervisor:}

Prof. Risto Orava, Ph.D.

Department of Physics

University of Helsinki

Finland

\section{Reviewers:}

Dr. Andrew Pilkington

Department of Physics \& Astronomy

University College London

UK

Dr. Rainer Schicker

Physikalisches Institut

University of Heidelberg

Germany

\section{Opponent:}

Prof. Valery Khoze, Ph.D., D.Sci.

Department of Physics

Durham University

UK

Cover image:

Event display of an exclusive $\gamma \gamma$ candidate recorded by CDF II (event 14704042, run 243808). Shown is the calorimeter lego plot (top), the central outer tracker (bottom left) and the r-z plot of the CDF detector (bottom right).

Report Series in Physics HU-P-D203

ISSN 0356-0961

ISBN 978-952-10-8092-0 (printed version)

ISBN 978-952-10-8093-7 (electronic version)

http://ethesis.helsinki.fi

Unigrafia

Helsinki 2013 
To Margit Fluch,

my physics teacher

who inspired me to

explore the microcosmos. 



\begin{abstract}
We have observed exclusive $\gamma \gamma$ production in proton-antiproton collisions at the Tevatron at $\sqrt{s}=1.96 \mathrm{TeV}$. We use data corresponding to $1.11 \pm 0.07 \mathrm{fb}^{-1}$ integrated luminosity taken by the Run II Collider Detector at Fermilab, with a trigger requiring two electromagnetic showers, each with transverse energy $E_{T}>2 \mathrm{GeV}$, and vetoing on hits in the forward beam shower counters. We select events with two electromagnetic showers, each with transverse energy $E_{T}>2.5 \mathrm{GeV}$ and pseudorapidity $|\eta|<1.0$, with no other particles detected in $-7.4<\eta<+7.4$. The two showers have similar $E_{T}$ and an azimuthal angle separation $\Delta \phi \sim \pi$; we find 34 events with exactly two matching charged particle tracks, agreeing with expectations for the QED process $p \bar{p} \rightarrow p+e^{+} e^{-}+\bar{p}$ by two photon exchange; and we find 43 events with no tracks. The latter are candidates for the exclusive process $p \bar{p} \rightarrow p+\gamma \gamma+\bar{p}$ by double pomeron exchange. We use the strip and wire chambers at the longitudinal shower maximum position within the calorimeter to measure a possible exclusive background from $\mathbb{P}+\mathbb{P} \rightarrow \pi^{0} \pi^{0}$, and conclude that it is consistent with zero and is $<15$ events at $95 \%$ C.L. The measured cross section is $\sigma_{\gamma \gamma \text {,excl }}\left(|\eta|<1, E_{T}(\gamma)>2.5 \mathrm{GeV}\right)=$ $2.48_{-0.35}^{+0.40}$ (stat) ${ }_{-0.51}^{+0.40}$ (syst) pb and in agreement with the theoretical predictions. This process is closely related to exclusive Higgs boson production $p p \rightarrow p+H+p$ at the Large Hadron Collider. The observation of the exclusive production of diphotons shows that exclusive Higgs production can happen and could be observed with a proper experimental setup.
\end{abstract}




\section{Acknowledgements}

First of all, I would like to express my gratitude to my supervisor Prof. Risto Orava who has provided me with the opportunity to conduct my postgraduate studies in an highly interesting research field within an international collaboration. He always trusted me and supported me throughout my studies. I am very grateful to Dr. Michael Albrow who suggested to me the search for exclusive diphotons at the Tevatron and was my adviser in the course of this work.

I would like to thank Dr. Rainer Schicker and Dr. Andrew Pilkington for reviewing the thesis and giving constructive criticism to improve it.

Furthermore, I would like to thank Prof. Valery Khoze not only for being opponent for my thesis defense but also for discussions about theoretical details. I thank Dr. Lucian Hardland-Lang for providing the excellent software to simulate the exclusive diphotons and always offering support and Dr. Pavel Murat for helping me with the CDF reconstruction software.

I am thankful to Doc. Kenneth Österberg, Prof. Jerry Lämsä, Dr. Francisco Garcia, Dr. Tuula Mäki and Dipl. Phys. Alexander Winkler for reading parts of my thesis and giving helpful comments. Additionally I would like to mention that Kenneth's excellent lectures were extremely helpful for practical reasons.

I am very grateful to M.Sc. Stewart Makkonen-Craig for often helping me in language matters and to Dr. Martin Fluch for teaching me how to use computers in a smarter way.

I thank all my colleges from the CDF Collaboration and especially from the Helsinki Institute of Physics with whom I had great discussions whether it was physics or something else.

The financial support to my research from the Magnus Ehrnrooth foundation is greatly acknowledged.

Finally, most importantly and certainly not least, I would like to express my deepest gratitude to Tiina, for her love, support and encouragement. This work would have not been possible without her! 


\section{Author's Contribution}

This dissertation presents the work the author carried out in the field of experimental particle physics during the years 2008 till 2011. This monograph describes in all details the search for central exclusive production of diphotons at the Tevatron protonantiproton collider. The result was published as first observation:

- T. Aaltonen et al. (CDF Collaboration), Observation of Exclusive $\gamma \gamma$ Production in $p \bar{p}$ Collisions at $\sqrt{s}=1.96$ TeV, Phys. Rev. Lett. 108, 081801 (2012).

The author has carried out the entire analysis in all details and has written the monograph all by himself.

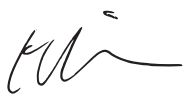

Erik Brücken 


\section{Contents}

Chapter 1. Introduction 1

Chapter 2. Theoretical Fundamentals 5

1. Standard Model Physics 5

1.1. Short Review of the Standard Model 5

1.2. Problems and Extensions 14

2. Strong Interactions and Diffraction at High Energies 16

2.1. What is Diffraction? 19

2.2. Soft Diffraction 24

2.3. The Pomeron and QCD 32

2.4. Central Exclusive Diffraction and the KMR Model 36

3. Photo Induced Central Exclusive Production 41

$\begin{array}{lll}\text { Chapter 3. Experimental Setup } & 43\end{array}$

1. Fermilab 43

1.1. Accelerator Complex 43

2. Collider Detector at Fermilab 46

2.1. Coordinate System 49

2.2. Tracking System 50

2.3. Calorimetry 53

2.4. Forward Detectors $\quad 59$

2.5. Additional Detection Devices 64

2.6. Trigger $\quad 65$

2.7. Data Quality and Reconstruction $\quad 67$

Chapter 4. Short Review of CEP Searches at Hadron Colliders 71

1. Early CEP Searches 71

2. CEP Searches at the Tevatron 73

2.1. Exclusive Dijet Production 74

2.2. Exclusive Electron-Positron Pair Production 74

2.3. Exclusive Charmonium and Muon Pair Production 74

2.4. Search for Exclusive $Z$ Production $\quad 75$

2.5. Early Exclusive Photon Pair Production 75 
Chapter 5. Analysis Methodologies

1. General Description of Analysis $\quad 77$

1.1. Cross Section Evaluation $\quad 77$

1.2. Statistical Methods 80

1.3. Data, Events and Variables 82

2. Physics Simulation 83

2.1. Events Generators 84

2.2. CDF Detector Simulation 86

3. Analysis Tools and Computing Framework 87

Chapter 6. Search for Exclusive Photon-Pair Production 89

1. Selection of Exclusive Electromagnetic Showers 89

1.1. The DIFF_DIPHOTON2 Trigger 89

1.2. Preselection of EM Events 93

1.3. Exclusive Filter 96

1.4. Post-selection of EM Exclusive Events 105

2. Exclusive Electron-Positron Pair Control Channel 110

2.1. Selection of Candidates 110

2.2. Radiation Losses 111

2.3. Final Exclusive Electron-Positron Pair Sample 112

$\begin{array}{ll}\text { 3. Exclusive Diphotons } & 117\end{array}$

3.1. Selection of Candidates 117

$\begin{array}{ll}\text { 3.2. Conversion Losses } & 117\end{array}$

3.3. Final Exclusive Diphoton Sample 118

4. Backgrounds 122

4.1. Neutral Pion Background 122

4.2. Dissociation Background 126

4.3. Other Background 130

5. Results and Discussion 131

5.1. Cross Section of the Exclusive Electron-Positron Pair Production 131

5.2. Cross Section of the Exclusive Photon Pair Production 132

$\begin{array}{lll}\text { Chapter 7. Conclusion and Outlook } & 137\end{array}$

$\begin{array}{ll}\text { Appendix A. Kinematics } & 139\end{array}$

$\begin{array}{lll}\text { Appendix B. Event Displays } & 147\end{array}$

$\begin{array}{ll}\text { Bibliography } & 151\end{array}$ 


\section{Abbreviations}

ABCDHW Ames - Bologna - CERN - Dortmund - Heidelberg - Warsaw collaboration

ADC Analog-to-digital converter

AFS Axial field spectrometer

ARCGM Aachen - Riverside - CERN - Genoa - Munich collaboration

ATLAS A Toroidal LHC Apparatus

BFKL Balitsky-Fadin-Kuraev-Lipatov equations

BSC Beam shower counter

CCHK CERN - Collège de France - Heidelberg - Karlsruhe collaboration

CCR Central crack chambers

CDF Collider Detector at Fermilab

CEM Central electromagnetic calorimeter

CEP Central exclusive production

CERN European organization for nuclear research

CES Central electromagnetic shower maximum detector

CHA Central hadronic calorimeter

CHOV CERN - Hamburg - Orsay - Vienna collaboration

CLC Cherenkov Luminosity Counter

CMP Central muon upgrade

CMS Compact Muon Solenoid

CMU Central muon detector

CMX Central muon extension

COT Central outer tracker

CPR Central pre-radiate chambers

$\mathrm{CPU} \quad$ Central processing unit

CSP Central muon scintillator

CSX Central scintillator extension

$\mathrm{D} \emptyset \quad$ D Zero experiment at Fermilab

DAQ Data acquisition system

DGLAP Dokshitzer-Gribov-Lipatov-Altarelli-Parisi equations

EM Electromagnetic

EPA Equivalent photon approximation

EWK Electroweak

IMU Intermediate muon detectors

IP Interaction point 


\begin{tabular}{|c|c|}
\hline ISL & Intermediate silicon layer \\
\hline ISR & Intersecting storage rings \\
\hline KMR & Khoze - Martin - Ryskin collaboration \\
\hline KRYSTHAL & Khoze - Ryskin - Stirling - Harland-Lang collaboration \\
\hline L00 & Layer 00 \\
\hline $\mathrm{LHC}$ & Large Hadron Collider \\
\hline LLA & Leading log approximation \\
\hline $\mathrm{LO}$ & Leading order \\
\hline $\mathrm{MC}$ & Monte Carlo simulation \\
\hline MET & Missing transverse energy \\
\hline MI & Main injector \\
\hline MIP & Minimum ionizing particle \\
\hline MRST & Martin - Roberts - Stirling - Thorne collaboration \\
\hline MSTW & Martin - Stirling - Thorne - Watt collaboration \\
\hline NLO & Next to leading order \\
\hline PDF & Parton density function \\
\hline PEM & Plug electromagnetic calorimeter \\
\hline PES & Plug electromagnetic shower maximum detector \\
\hline PHA & Plug hadronic calorimeter \\
\hline PMT & Photomultiplier tube \\
\hline PPR & Plug pre-radiate chambers \\
\hline pQCD & perturbative quantum chromodynamics \\
\hline QCD & Quantum chromodynamics \\
\hline QED & Quantum electrodynamics \\
\hline RPS & Roman Pot spectrometers \\
\hline SFM & Split field magnet spectrometer \\
\hline $\mathrm{SM}$ & Standard model of particle physics \\
\hline $\mathrm{Sp} \overline{\mathrm{p}} \mathrm{S}$ & CERN proton-antiproton collider \\
\hline SUSY & Supersymmetry \\
\hline SVX & Silicon vertex detector \\
\hline TOF & Time-of-flight detector \\
\hline TOTEM & $\begin{array}{l}\text { Total cross section, elastic scattering and diffraction dissociation at the } \\
\text { Large Hadron Collider }\end{array}$ \\
\hline UA1 & Experiment at the CERN super proton synchrotron \\
\hline UA2 & Experiment at the CERN super proton synchrotron \\
\hline UA8 & Experiment at the CERN super proton synchrotron \\
\hline WHA & End-Wall hadronic calorimeter \\
\hline WLS & Wavelength shifting fibers \\
\hline
\end{tabular}




\section{CHAPTER 1}

\section{Introduction}

The central exclusive process $p \bar{p} \rightarrow p+\gamma \gamma+\bar{p}$, where "+" means a large rapidity gap $(\Delta y \gtrsim 4)$, and "exclusive" means there are no other particles produced. In other words "exclusive" means that all energy lost by the colliding (anti)protons goes into the production of the central system, a diphoton system in our case. This is probably the cleanest strong interaction process apart from elastic scattering, which makes it very interesting from a quantum chromodynamics (QCD) point of view. Using an equivalent, somewhat historical notation, the process can be written as $\mathbb{P}+\mathbb{P} \rightarrow \gamma+\gamma$, where $\mathbb{P}$ stands for the "pomeron".

The dominant process theoretically $[\mathbf{1}-\mathbf{3}]$ is gluon fusion through quark loops, $g g \rightarrow q \bar{q} \rightarrow \gamma \gamma$. The two gluons have to be in a color singlet state. The process can be exclusive if (a) another gluon is exchanged to cancel the color (b) there are no other parton-parton interactions (c) there is no gluon radiation that can create hadrons ${ }^{1}$ and (d) neither (anti)proton underwent diffraction dissociation such as $p \rightarrow p \pi \pi, n \pi^{+}$. All these requirements reduce the cross section by a large factor, involving challenging QCD effects. Item (a) involves the two-gluon (unintegrated) distribution function $G\left(x_{1}, x_{2}\right)$ including correlations between low- $x$ gluons in the proton; (b) is called the "rapidity gap survival factor", $S^{2}$, and can be best estimated from other diffractive cross sections; (c) is called Sudakov suppression; and (d) is calculable from Regge theory but not from QCD, or it can be taken from diffractive data. The pomeron $\mathbb{P}$, the Regge $t$-channel exchange (see Ref. [4] for a review) with vacuum quantum numbers, is well known in Regge theory and should eventually be calculable from QCD, as the leading order pomeron is a pair of low- $x$ gluons. This is however challenging theoretically because $Q^{2}$ is small and so the strong coupling $\alpha_{s}$ becomes large, and perturbative calculations break down. Given all these theoretical uncertainties the (very few) predictions are typically uncertain by a factor $\underset{\div 3}{\times 3}$.

This process $p \bar{p} \rightarrow p+\gamma \gamma+\bar{p}$ is quite remarkable. The initial state has only strong interactions and the final state has only electromagnetic interactions. Therefore there must be an intermediate state that has both, and the only Standard Model possibility

\footnotetext{
${ }^{1} \mathrm{~A}$ single gluon cannot be radiated, there must be at least two.
} 
is a (sum of) light quark loop(s). Hence a precision measurement ${ }^{2}$ could be a window on other loops such as squarks $\tilde{q}$ and rigorously help constraining the theory inputs such as the unintegrated gluon PDFs, the Sudakov suppression, etc.

As discussed already, the dominant process for $p \bar{p} \rightarrow p+\gamma \gamma+\bar{p}$ is $g g \rightarrow \gamma \gamma$ through a quark loop, which is the same QCD diagram as for the CEP of the Higgs boson $p p \rightarrow p+H+p$, which has $g g \rightarrow H$ through a top quark loop. Thus the observation of exclusive $\gamma \gamma$ production at the Tevatron means that exclusive Higgs boson production will be possible, and while that cross section is much too small at the Tevatron [5], it should be detectable at the LHC with an appropriate experimental setup. Many, but not all, of the theoretical uncertainties (the factors (a)-(d) above) cancel in the ratio of exclusive $\gamma \gamma / H$. However $Q^{2}$ is much lower in the $\gamma \gamma$ case, with small masses $(M(\gamma \gamma) \gtrsim 5 \mathrm{GeV})$ and therefore non-perturbative. Within typical factors of $\stackrel{\times 3}{\div 3}$ uncertainty, the Durham group predicted [1] $\sigma_{\gamma \gamma}$ (Tevatron, $E_{T}(\gamma)>5 \mathrm{GeV}$, $|\eta(\gamma)|<1.0)=38 \mathrm{fb}\left(\right.$ MRST99), and $\sigma_{H}(\mathrm{LHC} 7+7 \mathrm{TeV}, M(H)=120 \mathrm{GeV})=3 \mathrm{fb}$.

Other possible exclusive two-photon production processes are $\gamma \gamma \rightarrow \gamma \gamma$ (light-onlight scattering) where the virtual (off-shell) photons are radiated coherently off the (anti)protons [1]. This is expected to be lower by a factor $\sim 400$ at $E_{T}>5 \mathrm{GeV}$, and by an even bigger factor for lower $E_{T}(\mathrm{~min})$. Quark-antiquark annihilation, $q \bar{q} \rightarrow \gamma \gamma$ is also expected to be only $\sim 10^{-2}$ of the gluon fusion cross section in central exclusive production when $E_{T}(\min )>5 \mathrm{GeV}$, and to fall faster with $E_{T}$ than the other terms. This is mainly because the cancellation of the color (triplet) exchange has to be made by another quark, so the $t$-channel exchange is $q \bar{q}$, i.e. a virtual (Regge) meson or "reggeon". Reggeon exchanges (as their intercept $\alpha_{R}(t=0) \sim 0.5$, less than 1.0) decrease fast as the rapidity gaps $\Delta y$ increase, which is not the case for the pomeron $\left(\alpha_{\mathbb{P}}(0)>1\right)$ or photon $\left(\mathrm{J}_{\gamma}=1\right)$.

A possible background is central exclusive $\pi^{0} \pi^{0}$ production (i.e. $\mathbb{P}+\mathbb{P} \rightarrow \pi^{0} \pi^{0}$ ). This was also theoretically poorly understood (at the time of the analysis), and there is no relevant data except for exclusive $\pi^{+} \pi^{-}$production in $p p$-collisions at the much lower $\sqrt{s}=63 \mathrm{GeV}$, at non-zero $t$, and extending only to $M(\pi \pi) \sim 3 \mathrm{GeV} / \mathrm{c}^{2}$. Any extrapolation of that data to the CDF region would have a too large uncertainty to be useful. The Durham group expect the ratio $\mathrm{R}\left(\pi^{0} \pi^{0} / \gamma \gamma\right)$ to be "small", basically because the pions are extended objects (with a form factor that falls fast with $Q^{2}$ ).

Fortunately we can distinguish $\gamma$ from $\pi^{0}$ using the strip/wire proportional chambers (CES) near shower maximum in the electromagnetic calorimeter. The 2 photons from $\pi^{0}$ decay have a minimum opening angle $\theta_{\gamma \gamma}(\min )=2 m(\pi) / E(\pi)$ and cannot merge in our energy region. A $\pi^{0}$ can only give a single cluster if one photon falls in a "crack"

\footnotetext{
${ }^{2}$ This is beyond the scope of this study, but perhaps possible at the Large Hadron Collider
} 
$(\sim 8 \%)$, or the shower ranges out or does not start before the CES $(\sim 2 \%)$. These will be discussed in detail. An additional constraint comes from exclusive $\gamma+\pi^{0}$ being forbidden (by C-parity and spin), so only $\gamma \gamma$ and $\pi^{0} \pi^{0}$ are possible ${ }^{3}$.

Historically the exclusive diphoton process was first proposed in 2001 in a Letter of Intent [5] to look for exclusive Higgs boson production in CDF. The idea to look for exclusive Higgs boson production at hadron colliders was already earlier discussed by the Durham group [7, 8]. Khoze, Martin and Ryskin from Durham then discussed in addition the central exclusive production of $\gamma \gamma[\mathbf{9}]$ and concluded that the cross section would be too small to see at the Tevatron, but they later revised this conclusion [1].

In December 2004 we initiated a trigger to look for it. The trigger required 2 forward gaps, an area without particle activity, using the Beam Shower Counters, and 2 electromagnetic (EM) showers of $4 \mathrm{GeV}$ in the calorimeter. We found three candidate events with two back-to-back electromagnetic showers with a transverse energy $E_{T}>5 \mathrm{GeV}$ and no charged particle tracks together with 16 events that had tracks and agreed with expectations for the quantum electrodynamical (QED) process $p+\bar{p} \rightarrow p+e^{+} e^{-}+\bar{p}$ through the intermediate process $\gamma \gamma \rightarrow e^{+} e^{-}[\mathbf{1 0}]$. The latter process is precisely calculated and provides a good control of the analysis. The theoretical expectation for the exclusive $\gamma \gamma$ study, published in [11], was 0.8 events with an uncertainty factor $\stackrel{\times 3}{\div 3}$ (at that time), and therefore consistent with three candidates. The statistics were too low, and the background from $\pi^{0} \pi^{0}$ too uncertain, to claim an observation.

More recent and improved calculations by the Durham group, with the SUPERCHIC Monte Carlo, predicted [2] a $\gamma \gamma$ cross section at the lower $E_{T}(\gamma)>2.5 \mathrm{GeV},|\eta|<1.0$ of $1.42_{\div 2}^{\times 2} \mathrm{pb}($ MSTW08LO) at the Tevatron. The spread in their predictions comes largely from different gluon distribution functions (MRST99, MSTW08LO) [3]. The factor of $\sim 15$ increase expected by lowering the $E_{T}(\gamma)$ threshold obviously has the potential of upgrading the three-candidate search into an observation, as long as the background is small and understood.

We subsequently reduced the trigger threshold on the two EM showers from $4 \mathrm{GeV}$ to $2 \mathrm{GeV}$, which we could do without any prescaling (thanks to the Beam Shower Counter veto) and took more data. As the cross section falls steeply with the invariant mass of the photon-pair $M(\gamma \gamma)[\mathbf{1}]$, we find many more candidates. We are able to make a data-driven estimate of backgrounds, in particular $\pi^{0} \pi^{0}$. The information in the strip/wire chambers at the shower maximum position at six radiation lengths $\left(6 X_{0}\right)$ is consistent with all the events being $\gamma \gamma$, and we find the fraction of events which

\footnotetext{
${ }^{3}$ In principle central exclusive production of neutral mesons pairs $\eta \eta, \eta \eta^{\prime}$ and $\eta^{\prime} \eta^{\prime}$ could contribute, but as it is stated in $[6]$ these contributions to the $4 \gamma$ state are expected to be small.
} 
are $\pi^{0} \pi^{0}$ to be $<34 \%$ at $95 \%$ C.L. We also find exclusive $e^{+} e^{-}$events, consistent with the QED process we previously observed, which is a good control of the analysis procedures.

This thesis describes the analysis work on the new data set that has been done for the successful observation of the exclusive diphoton production in every detail. First in Chapter 2 the theoretical fundamentals and context for this process are given. The standard model will be shortly introduced with emphasis on the strong interaction theory at high energies. This is followed by a more detailed introduction to the world of diffraction in high energy hadron-hadron collisions. The key part of this chapter will be the description of the theoretical understanding of the exclusive diphoton production. Chapter 3 presents the experimental apparatus used to record the data that was used in this work. The description will emphasize on actual used components but nonetheless the complete CDF II detector will be mentioned to give the reader the idea of its full potential. The next Chapter will briefly review central exclusive production in hadron-hadron collider experiments prior to this study. Chapter 5 defines this analysis and introduces the methods and techniques used. The actual analysis consisting of the event selection, the estimation of efficiencies and acceptances as well as the discussion of possible backgrounds is covered by Chapter 6 . The thesis concludes with Chapter 7 in which the results of this work will be summarized and discussed also in context of implications to theory and future experimental studies.

For pure convenience of notation the Planck quantum $\hbar$ and the speed of light $c$ will be set to 1 throughout this work, $\hbar=c=1$. 


\section{CHAPTER 2}

\section{Theoretical Fundamentals}

\section{Standard Model Physics}

The standard model of particle physics is a great step towards Heisenberg's dream of a world-formula. It has been rigorously tested in various experiments in great precision over the past decades. It very successfully provides a detailed and unified picture of the quantum nature of our world. In detail it describes the origin of matter and the interplay with the fundamental forces in our universe, the electromagnetic, the weak and the strong force. A major shortcoming of the standard model is its inability to also include and describe the fourth known force, the gravity. In this chapter the standard model will be shortly reviewed. After the introduction to its construction some of its successes will be mentioned as well as some of its shortcomings.

\subsection{Short Review of the Standard Model}

From an experimental particle physicist point of view the Standard Model (SM) consists of a zoo of matter particles in combination with particles mediating the electromagnetic, weak and strong forces ${ }^{1}$. Table 2.1 and 2.2 show all known fundamental particles of the SM consisting of spin $1 / 2$ fermions building up the matter (Table 2.1) and of spin 1 bosons, the force carriers (Table 2.2). Mathematically the SM can be derived from the non-abelian symmetry group, $S U(3)_{C} \otimes S U(2)_{L} \otimes U(1)_{Y}$ with $C$ denoting the color charge, $Y$ the weak hypercharge and $L$ the fact that this part of the symmetry group only acts on left-handed fermions. It is shown that the SM is a fully renormalizable and gauge invariant theory.

Three generations of leptons and three generation of quarks are found. Whereas leptons only interact electroweakly, quarks couple additionally to the strong force. Quarks and leptons exist as left-handed doublets with non-zero weak isospin and right-handed singlets with zero weak isospin. Those matter particles can interact with each other via an exchange of gauge bosons. Table 2.2 shows the gauge bosons relevant for the electroweak and strong forces. The massless photons $(\gamma)$ and the massive vector

\footnotetext{
${ }^{1}$ The reader who is interested in more details is referred to numerous textbooks about particle physics and quantum field theories such as $[\mathbf{1 2}, \mathbf{1 3}]$.
} 
TABLE 2.1. Standard Model fermions. Weak isodouplets are combined in brackets. The primed quarks are mixed states due to a general Cabibbo-rotation.

\begin{tabular}{ccccc}
\hline & \multicolumn{3}{c}{ Generations } & \multicolumn{3}{c}{$S U(3)_{C} \otimes S U(2)_{L} \otimes U(1)_{Y}$} \\
\hline Quarks & $\left.\begin{array}{c}u \\
d^{\prime}\end{array}\right)_{L}$ & $\left(\begin{array}{c}c \\
s^{\prime}\end{array}\right)_{L}$ & $\left(\begin{array}{c}t \\
b^{\prime}\end{array}\right)_{L}$ & $\left(3,2, \frac{1}{3}\right)$ \\
& $u_{R}$ & $c_{R}$ & $t_{R}$ & $\left(3,1, \frac{4}{3}\right)$ \\
& $d_{R}$ & $s_{R}$ & $b_{R}$ & $\left(3,1, \frac{-2}{3}\right)$ \\
\hline Leptons & $\left(\begin{array}{c}e \\
\nu_{e}\end{array}\right)_{L}$ & $\left(\begin{array}{c}\mu \\
\nu_{\mu}\end{array}\right)_{L}$ & $\left(\begin{array}{c}\tau \\
\nu_{\tau}\end{array}\right)_{L}$ & $(1,2,-1)$ \\
& $e_{R}$ & $\mu_{R}$ & $\tau_{R}$ & $(1,1,-2)$ \\
\hline
\end{tabular}

TABLE 2.2. SM gauge bosons and the relevant gauge couplings.

\begin{tabular}{cccc}
\hline Gauge group & Gauge bosons & $S U(3)_{C} \otimes S U(2)_{L} \otimes U(1)_{Y}$ & Coupling constant \\
\hline$U(1)_{Y}$ & $B_{\mu}$ & $(1,1,0)$ & $g^{\prime}$ \\
$S U(2)_{L}$ & $W_{\mu}^{i}, i=1 \ldots 3$ & $(1,3,0)$ & $g$ \\
$S U(3)_{c}$ & $G_{\mu}^{a}, a=1 \ldots 8$ & $(8,1,0)$ & $g_{s}$ \\
\hline
\end{tabular}

bsosons $\left(W^{ \pm}, Z\right)$ mediate the electroweak force and the color charge carrying massless gluons (8 representations) the strong force.

Historically the combination of the electromagnetic and the weak interactions in the 60ies of the last century led to the idea of a complete theory of the nature, the SM. By introducing the Higgs mechanism to the electroweak (EWK) theory and adding the theory of the strong interactions, the SM was born.

1.1.1. Quantum Electrodynamics - a gauge field theory example. The quantum electrodynamics (QED) is the quantized and relativistic version of the classical electromagnetic field theory describing the interaction of charged particles with the electromagnetic field. The QED is a locally invariant gauge field theory based on the local abelian symmetry group $U(1)$. Along with the QED some thoretical terms and constructs are introduced that are important for a better understanding of the SM. The QED can be derived from the abelian group $U(1)$, represented by unitary $1 \times 1$ matrices. In quantum field theories the Lagrangian formalism is usually used to introduce the dynamics of the system similar as in classical mechanics. The Lagrangian for the Dirac fermion fields can be written as

$$
\mathcal{L}=i \bar{\psi} \gamma^{\mu} \partial_{\mu} \psi-m \bar{\psi} \psi
$$


with the fermionic fields $\psi$, the Dirac matrices $\gamma^{\mu}$ and the mass term $m$. This Lagrangian is invariant under the global gauge transformation $\psi \rightarrow e^{i \phi} \psi$. If the phase is a function of space-time $\phi(x)$ one can see that the Lagrangian is not invariant under such a local gauge transformation. One is left with an extra term,

$$
\mathcal{L} \rightarrow \mathcal{L}-\left(\partial_{\mu} \phi\right) \bar{\psi} \gamma^{\mu} \psi
$$

that can be eliminated by adding a new so-called gauge field $A_{\mu}$ to the Lagrangian that transforms locally as $A_{\mu} \rightarrow A_{\mu}-\partial_{\mu} \phi / q$, where $q$ is the charge of the interacting fermion. Before the QED Lagrangian is complete one needs to add a term describing the introduced spin 1 vector field, $\mathcal{L}=F^{\mu \nu} F_{\mu \nu} / 4$, with $F^{\mu \nu} \equiv \partial^{\mu} A^{\nu}-\partial^{\nu} A^{\mu}$. To hold the local gauge invariance the vector field has to be massless. Which gives the total QED Lagrangian,

$$
\mathcal{L}=i \bar{\psi} \gamma^{\mu} \partial_{\mu} \psi-m \bar{\psi} \psi-q\left(\bar{\psi} \gamma^{\mu} \psi\right) A_{\mu}-\frac{1}{4} F^{\mu \nu} F_{\mu \nu}
$$

By introducing the covariant derivative $\mathcal{D}_{\mu}=\partial_{\mu}+i q A_{\mu}$, the Langrangian can be simplified,

$$
\mathcal{L}=i \bar{\psi} \gamma^{\mu} \mathcal{D}_{\mu} \psi-m \bar{\psi} \psi-\frac{1}{4} F^{\mu \nu} F_{\mu \nu}
$$

The local phase transformation is a representation of the unitary symmetry group $U(1)$. The free parameter of this group can be seen as the massless bosonic mediator of the electromagnetic field, the photon.

Far more complicated to describe is the theory of the weak interaction with its massive mediators the $W^{ \pm}$and $Z$ vector bosons.

1.1.2. Electroweak Symmetry Breaking and the Higgs Boson. Having seen the principle of a gauge field theory in case of the QED, one now starts from the full electroweak Lagrangian of the SM. Subsequently some details are explained how the SM is constructed and what parameters define it.

The full electroweak Lagrangian can be written as

$$
\begin{gathered}
\mathcal{L}=i \bar{f}_{L} \gamma^{\mu}\left(\partial_{\mu}+\frac{i g}{2} \boldsymbol{\tau} \cdot \boldsymbol{W}_{\mu}+\frac{i g^{\prime} Y}{2} B_{\mu}\right) f_{L}+i \bar{f}_{R} \gamma^{\mu}\left(\partial_{\mu}+\frac{i g^{\prime} Y}{2} B_{\mu}\right) f_{R} \\
\quad-\frac{1}{4} \boldsymbol{W}_{\mu \nu} \boldsymbol{W}^{\mu \nu}-\frac{1}{4} B_{\mu \nu} B^{\mu \nu} \\
+\left[\left(\partial_{\mu}+\frac{i g}{2} \boldsymbol{\tau} \cdot \boldsymbol{W}_{\mu}+\frac{i g^{\prime} Y}{2} B_{\mu}\right) \phi\right]^{\dagger}\left[\left(\partial_{\mu}+\frac{i g}{2} \boldsymbol{\tau} \cdot \boldsymbol{W}_{\mu}+\frac{i g^{\prime} Y}{2} B_{\mu}\right) \phi\right]-V(\phi) \\
\quad-g_{l}\left(\bar{l}_{L} \phi l_{R}+\bar{l}_{R} \phi^{\dagger} l_{L}\right)+g_{1} \bar{q}_{L} \phi_{c} u_{R}+g_{2} \bar{q}_{L} \phi d_{R}+g_{1}^{\dagger} \bar{u}_{R} \phi_{c}^{\dagger} q_{L}+g_{2}^{\dagger} \bar{d}_{R} \phi^{\dagger} q_{L} .
\end{gathered}
$$

The first line represents the kinetic terms of the left-handed lepton and quark doublets $\left(f_{L}\right)$ and right-handed lepton and quark singlets $\left(f_{R}\right)$ as well as its couplings with 
the electroweak gauge bosons. The second line shows the kinetic and self interaction terms of the electroweak gauge bosons. The third line shows the couplings of the gauge bosons to the Higgs field and the free kinetic terms of this scalar field. Also included are the mass terms of the electroweak gauge bosons and the Higgs boson. The fourth line shows the mass terms of the fermions including the coupling to the Higgs field and the quark mixing. Here $l_{L}$ denotes the left-handed lepton doublet, $l_{R}$ the right-handed lepton singlet, $g_{l}$ an arbitrary coupling. In case of the quarks one has the left-handed quark doublet $q_{L}$, the right handed singlet for the up-type quarks $u_{R}$ and the down-type quark $d_{R}$. Right-handed up-type quark singlets couple to a conjugated form of the Higgs doublet $\left(\phi_{c}\right)$.

For discussion the SM Lagrangian will be split up into parts to understand its meanings. As noted the first two lines represent the free kinetic terms of the spin $1 / 2$ fermions and the kinetic and self interaction terms of the electroweak gauge bosons and their couplings to the fermionic sector. Essentially this represents the Langrangian of the electroweak model based on the non-abelian $S U(2) \otimes U(1)$ symmetry group. The $S U(2)$ part of the symmetry group is represented by $2 \times 2$ matrices with determinant 1 . Analog to the $U(1)$ one has local $S U(2)$ transformations, $\psi \rightarrow e^{-i \boldsymbol{\alpha}(x) \cdot \boldsymbol{T}} \psi$. Here $\boldsymbol{T}$ is the $S U(2)$ group generator that can be expressed in terms of the $2 \times 2$ Pauli matrices $\boldsymbol{T}_{i}=\boldsymbol{\tau}_{i} / 2$. Under the $S U(2) \otimes U(1)$ gauge group the massless fermion fields transform as,

$$
\psi \rightarrow e^{-i\left(\frac{\alpha(x) \cdot \boldsymbol{\tau}}{2}+\beta(x) Y\right)} \psi .
$$

It has to be noted that this transformation only acts on the left-handed part of the fermions $f_{L}$. To have the Lagrangian invariant under this transformation rule one needs to add, as in the QED case, gauge fields that compensate the invariant terms. Convenient is to replace the partial derivatives by the covariant derivative which includes the $U(1)$ part of the electroweak model,

$$
\mathcal{D}_{\mu}=\partial_{\mu}+\frac{i g}{2} \boldsymbol{\tau} \cdot \boldsymbol{W}_{\mu}+\frac{i g^{\prime} Y}{2} \cdot B_{\mu}
$$

The term in the middle $(S U(2))$ only acts on left-handed fermions. One also needs to add the free kinetic term of the gauge fields $\boldsymbol{W}_{\mu \nu}$ and $B_{\mu \nu}$, with

$$
\boldsymbol{W}_{\mu \nu}=\partial_{\mu} \boldsymbol{W}_{\nu}-\partial_{\nu} \boldsymbol{W}_{\mu}-g \boldsymbol{W}_{\mu} \times \boldsymbol{W}_{\nu}
$$

and

$$
B_{\mu \nu}=\partial_{\mu} B_{\nu}-\partial_{\nu} B_{\mu}
$$

Which gives the full electroweak Lagrangian as shown in the first two lines of Equation (5). So far the local invariance of the Lagrangian is conserved because of the introduced massless gauge fields. In detail there are two charged gauge field Eigenstates 


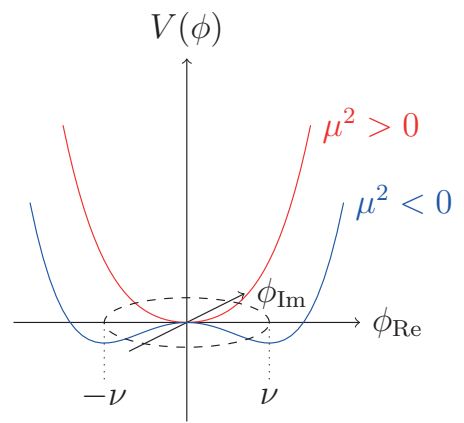

Figure 2.1. The potential of the scalar particle Lagrangian in Equation (13) (arbitrary scale).

$W_{\mu}^{1} W_{\mu}^{2}$ and two neutral ones $W_{\mu}^{3}$ and $B_{\mu}$. The physical electroweak gauge bosons $W_{ \pm}$ are a mixture of the the charged Eigenstates

$$
W_{\mu}^{ \pm}=\frac{1}{\sqrt{2}}\left(W_{\mu}^{1} \mp i W_{\mu}^{2}\right) .
$$

In case of the neutral physical gauge bosons $A_{\mu}$ and $Z_{\mu}$ there is a mixture of the neutral gauge field Eigenstates depending on the coupling of the electromagnetic and the weak forces in form of the Weinberg angle $\Theta_{W}$,

$$
\left(\begin{array}{l}
A_{\mu} \\
Z_{\mu}
\end{array}\right)=\left(\begin{array}{cc}
\cos \Theta_{W} & \sin \Theta_{W} \\
-\sin \Theta_{W} & \cos \Theta_{W}
\end{array}\right)\left(\begin{array}{c}
B_{\mu} \\
W_{\mu}^{3}
\end{array}\right)
$$

with

$$
\cos ^{2} \Theta_{W}=\frac{g^{2}}{g^{2}+g^{\prime 2}} \quad \text { and } \quad \sin ^{2} \Theta_{W}=\frac{g^{\prime 2}}{g^{2}+g^{\prime 2}} .
$$

Due to experimental results one knows that the charged physical vector bosons $W^{ \pm}$ and the neutral physical vector boson $Z$ cannot be massless as the photon. However, adding a mass term to the Lagrangian would violate its gauge invariance. This problem can be overcome by introducing the so-called Higgs mechanism to the electroweak model that leads to line three of the SM Lagrangian in Equation (5).

One starts with a complex scalar field with a Lagrangian,

$$
\mathcal{L}=\left(\partial_{\mu} \phi\right)^{\dagger}\left(\partial^{\mu} \phi\right)-\mu^{2} \phi^{\dagger} \phi-\lambda\left(\phi^{\dagger} \phi\right)^{2} .
$$

Here $\phi$ is a $S U(2)$ doublet of two complex scalar fields,

$$
\phi=\left(\begin{array}{c}
\phi^{+} \\
\phi^{0}
\end{array}\right)=\frac{1}{\sqrt{2}}\left(\begin{array}{c}
\phi_{1}+i \phi_{2} \\
\phi_{3}+i \phi_{4}
\end{array}\right)
$$

The potential of the complex scalar fields, $V(\phi)=\mu^{2} \phi^{\dagger} \phi+\lambda\left(\phi^{\dagger} \phi\right)^{2}$, as shown in Figure 2.1, shows the feature of a spontaneously broken symmetry if the arbitrary 
constants $\lambda>0$ and $\mu^{2}<0$. One speaks of a spontaneously broken symmetry if the Langrangian stays invariant under a group symmetry transformation but not the vacuum state. There is a degenerate global minimum energy state at $\nu=\sqrt{-\mu^{2} / \lambda}$. Choosing conveniently that only the neutral part of the complex scalar field acquires a non-zero vacuum expectation value whereas the other charged field vanishes one can write the minimum energy state of the neutral part as

$$
\phi_{0}=\frac{1}{\sqrt{2}}\left(\begin{array}{l}
0 \\
\nu
\end{array}\right)
$$

One can define a new field $\eta(x)$ fluctuating around the vacuum state ${ }^{2}$. As a result

$$
\phi(x)=\frac{1}{\sqrt{2}}\left(\begin{array}{c}
0 \\
\nu+\eta(x)
\end{array}\right) .
$$

Using the covariant derivative from the electroweak model Equation (7) in the Lagrangian Equation (13) and substitute Equation(16) one obtains the following Lagrangian,

$$
\begin{aligned}
\mathcal{L}= & \frac{1}{2} \partial_{\mu} \eta \partial^{\mu} \eta+\mu^{2} \eta^{2}-\frac{1}{4} \boldsymbol{W}_{\mu \nu} \boldsymbol{W}^{\mu \nu}-\frac{1}{4} B_{\mu \nu} B^{\mu \nu} \\
& +\frac{1}{8} \nu^{2} g^{2}\left(\left(W_{\mu}^{1}\right)^{2}+\left(W_{\mu}^{2}\right)^{2}\right)+\frac{1}{8} \nu^{2}\left(\left(g W_{\mu}^{3}-g^{\prime} B_{\mu}\right)^{2}\right),
\end{aligned}
$$

to which is also added the kinetic and self interacting terms of the electroweak gauge fields. One can immediately see from the second term that the scalar field obtained a mass $m_{\text {scalar }}=\sqrt{-2 \mu^{2}}=\sqrt{2 \nu^{2} \lambda}$. Remembering the mixing of the $S U(2)$ gauge bosons (Equation (10)) one can see that the $W_{\mu}^{ \pm}$vector bosons now have acquired a mass of $m_{W}=\nu g / 2$. The last term represents the neutral gauge boson sector that mixes according to Equation (11). It turns out that only the physical $Z_{\mu}$ field obtains a mass term of $m_{Z}=\nu \sqrt{g^{2}+g^{\prime 2}} / 2$ but not the $A_{\mu}$ field that is interpreted as the electromagnetic field with its massless photon. The masses between the $Z$ and $W^{ \pm}$vector bosons are directly related via the weak mixing angle, $m_{W}=m_{Z} \cos \Theta_{W}$. Very recently the CDF Collaboration published the latest and presently most precise mass measurement of the $W^{ \pm}$vector boson with a measured mass of $m_{W}=80.387 \pm 0.019 \mathrm{GeV}$ [14]

In a similar fashion, masses to fermions can also be introduced using the same Higgs doublet (see line four of Equation (5)). Additionally one should mention that the coupling constants for the leptons and quarks are arbitrary which means that their masses cannot be calculated from first principles. The actual mass of the SM Higgs boson is unkown as well, despite some theoretical boundaries. Recently the

\footnotetext{
${ }^{2}$ It has to be added that also an other field, $\xi(x)$, shows up expanding around the imaginary part of the potential with its vacuum state at zero. This will result in a field accompanied by a massless boson, known as the Goldstone boson. By choosing the right gauge, the Goldstone boson can be removed without changing the invariance of the Lagrangian.
} 
two major experiments at Large Hadron Collider (LHC), ATLAS and CMS published their results on the search for the Higgs boson. They observed a bosonic particle with a mass around $125 \mathrm{GeV}[\mathbf{1 5}, \mathbf{1 6}]$. Whether this is the SM Higgs boson or some boson described by beyond the SM physics more studies have do be done. Especially challenging at hadron colliders is the determination of its quantum numbers. To anticipate, observing this boson via central exclusive production $p p \rightarrow p+X+p$ could be of great help in detecting most of its quantum numbers. The quantum numbers of the system $X$ are strongly restricted to be $I^{G} J^{P C}=0^{+}(\text {even })^{++}[\mathbf{1 7}]$. More about the selection rules will be discussed later. In the following the part of the SM describing the strong interaction will be briefly introduced.

1.1.3. Quantum Chromodynamics. The strong interaction can be formulated as a gauge field theory, called quantum chromodynamics (QCD), derived from the $S U(3)_{C}$ group. The Lie group with its eight free parameters is able to describe the strong interactions with its six quark flavors and their color charge, at sufficiently high energies. Two phenomenological properties are closely connected to this theory, namely asymptotic freedom and confinement that will be discussed below. One can start from the free Lagrangian Equation (1), this time for quark fields and its color charge. Here the four component Dirac spinor $\psi$ is now replaced by a three component vector with each component being itself a Dirac spinor with a different color charge:

$$
\mathcal{L}=\bar{\psi}_{i}\left(i \gamma^{\mu} \partial_{\mu}-m\right)_{i j} \psi_{j}
$$

The Lagrangian is globally invariant under the transformation $\psi \rightarrow U \psi$ with $U$ being unitary $3 \times 3$ matrices. The transformation can be written in the form

$$
\psi \rightarrow e^{-\frac{i g_{s}}{2} \boldsymbol{\lambda} \cdot \phi} \psi
$$

where $\boldsymbol{\lambda}$ is a vector of the eight generators of a suitable fundamental $S U(3)$ representation, $\phi$ a vector of arbitrary parameters and $g_{s}$ the coupling constant. The generators are chosen in the form of the Gell-Mann matrices. The matrices fulfill the commutation relation $\left[\lambda_{a}, \lambda_{b}\right]=2 i f_{a b c} \lambda_{c}$ with $f_{a b c}$ being antisymmetric structure constants with respect to the indices. To have a locally invariant Lagrangian when $\phi \rightarrow \phi(x)$, we replace, as usually, the derivative by the covariant derivative $\partial_{\mu} \rightarrow \mathcal{D}_{\mu}$,

$$
\mathcal{D}_{\mu}=\partial_{\mu}+\frac{i g_{s}}{2} \boldsymbol{\lambda} \cdot \boldsymbol{G}_{\mu}
$$

As in the QED case one introduces gauge fields $\boldsymbol{G}$, eight to be precise. These spin 1 fields called gluons are massless. Additionally one needs to add the free kinetic terms of the new gauge fields to the Lagrangian that now becomes,

$$
\mathcal{L}=\bar{\psi}_{i}\left(i \gamma^{\mu} \mathcal{D}_{\mu}-m\right)_{i j} \psi_{j}-\frac{1}{4} G_{\mu \nu}^{a} G_{a}^{\mu \nu},
$$


with the gluon field tensor

$$
G_{\mu \nu}^{a}=\partial_{\mu} G_{\nu}^{a}-\partial_{\nu} G_{\mu}^{a}-g_{s} f_{a b c} G_{\mu}^{a} G_{\nu}^{a} .
$$

For simplicity the additional gauge-fixing terms and the Faddeev-Popov ghost terms are skipped here. Those are added to the Lagrangian for technical reason. It turns out that the gluons carry color charge and are, in contrast to the QED mediator, self-interacting. Gluons couple not only to quarks but also to themselves, meaning similar to a quark radiating a gluon, a gluon can emit or absorb another gluon. An important characteristic of the QCD, in contrast to the QED, is the effective coupling that is decreasing with increasing momentum transfer. The running coupling constant is based on the renormalization group theory. The problem of calculating higher order processes including loops are divergences one cannot get hold on without introducing some unphysical cut off. The rather technical procedure of renormalization helps out to get rid of those infinities by redefining gauge field quantities. In QED one can redefine the coupling by introducing the bare coupling. The coupling measured is the bare coupling shifted by vacuum polarization, the photon self-energy contribution at lowest order. Summing up all higher order photon self-energy contributions one ends up with Equation (23). It shows the QED running coupling $\alpha$ in leading order that is increasing over increasing momentum transfer $Q^{2}$ starting from the fine-structure constant $\alpha(0) \approx 1 / 137$ up to the Landau pole.

$$
\alpha\left(Q^{2}\right)=\frac{\alpha\left(\mu^{2}\right)}{1-\frac{\alpha\left(\mu^{2}\right)}{3 \pi} \ln \left(\frac{Q^{2}}{\mu^{2}}\right)}
$$

In case of the QCD one has a somewhat more complicated picture due to the self interaction capabilities of the gluons. There are not only quark anti-quark loops but also gluon loops contributing to the gluon self-energy. The gluon loops have an opposite effect to the color charge, one speaks about anti-screening. Summing up all those contributions leads to a formula for the running coupling similar to the QED one,

$$
\alpha_{s}\left(Q^{2}\right)=\frac{\alpha_{s}\left(\mu^{2}\right)}{1+\frac{\alpha_{s}\left(\mu^{2}\right)}{12 \pi}(11 n-2 f) \ln \left(\frac{Q^{2}}{\mu^{2}}\right)},
$$

with $n$ being the number of colors and $f$ the number of flavors. In the present SM one has three colors and and six quark flavors as already mentioned. This configuration $(11 n-2 f>0)$ and the fact that one deals with a non-abelian gauge field theory based on the $S U(3)$ group leads to the effect of asymptotic freedom and confinement. The denominator is increasing for increasing momentum transfer that results in an asymptotically vanishing coupling constant $\alpha_{s}$. Phenomenologically, one has so to say 


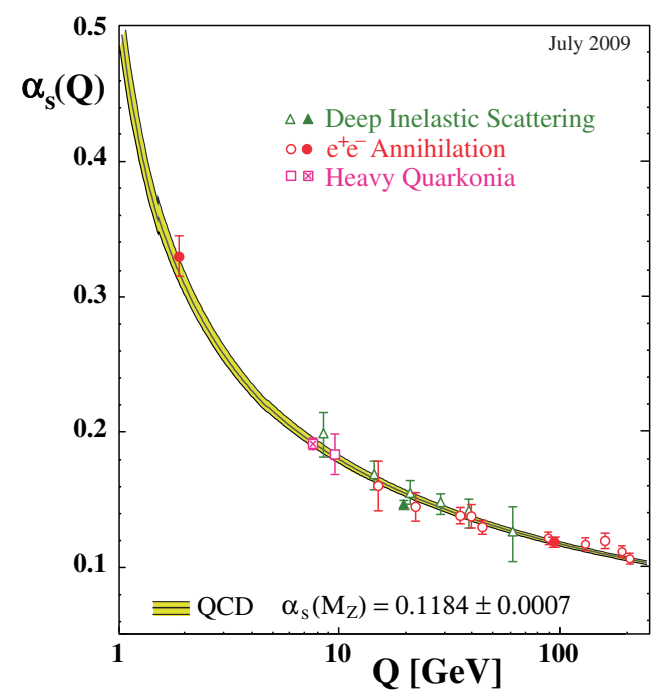

FiguRE 2.2. Measurements of the strong coupling constant $\alpha_{s}$ over the energy scale $\mathrm{Q}[\mathbf{1 8}]$. Note the large variation of $\alpha_{s}$ compared to the only minimal changing electromagnetic coupling $\alpha$ from $\alpha(0)=1 / 137$ to $\alpha\left(m_{Z}\right)=1 / 129$.

quasi free quarks at small distances or large momentum transfer. Measurements of the running coupling $\alpha_{s}$ over energy scale $Q$ agree well with the predicted behavior, shown in Figure 2.2. Towards smaller momentum transfer however one finds a steep rising of $\alpha_{s}$. This leads to a breakdown of the QCD perturbation theory when $\alpha_{s}$ approaches 1 . A new variable $\Lambda_{\mathrm{QCD}}$ is introduced showing approximately the range perturbative operations are applicable. Rewriting Equation (24) one gets,

$$
\alpha_{s}\left(Q^{2}\right)=\frac{12 \pi}{(11 n-2 f) \ln \left(\frac{Q^{2}}{\Lambda_{\mathrm{QCD}}}\right)} .
$$

Now there is only one free parameter that is defined by measurement and indicates the scale where perturbative expansions get out of control, typically the mass scale of hadrons $\sim 200 \mathrm{MeV}$. At those low energy scales quark and colors are hidden within hadron objects. Here one speaks about confinement or more precise color-confinement. Quarks cannot be isolated and are always bound in hadronic states that are colorless. Trying to separate quarks from a bound state using a sufficient amount of energy leads to breaking up by spontaneously generated quark anti-quark pairs. This process, also called hadronization, results in quarks or gluon jets instead of observable quarks or gluons in an experiment. 


\subsection{Problems and Extensions}

The SM has become a theoretical model of huge success. It was not only able to predict key features, for example the massive electroweak vector bosons $W^{ \pm}$and $Z$, or the heaviest and latest found quark, the top-quark, but also confirms a huge number of experimentally determined quantities such as particle decay channels, their guantum numbers, etc. However, not only that the SM includes a large number of free parameters only determined by experiment (see Table 2.3), there also exist serious flaws which strongly suggest that something beyond the SM must exist.

TABLE 2.3. Free SM parameters determined by experiments. Note: Neutrino oscillations are not considered here.

\begin{tabular}{lllllll}
\hline Parameter & \multicolumn{7}{c}{ Symbol } \\
\hline Quark masses & $m_{u}$ & $m_{d}$ & $m_{u}$ & $m_{d}$ & $m_{u}$ & $m_{d}$ \\
Lepton masses & $m_{e}$ & $m_{\mu}$ & $m_{\tau}$ & & & \\
CKM (angles + phase) & $\theta_{12}$ & $\theta_{23}$ & $\theta_{13}$ & $\delta$ & & \\
Couplings & $g^{\prime}$ & $g$ & $g_{s}$ & & & \\
Higgs & $m_{h}$ & $\nu$ & & & \\
QCD vacuum angle & $\theta_{\mathrm{QCD}}$ & & & \\
\hline
\end{tabular}

Among those flaws, the most prominent is that the SM is not including the graviton. The theory of gravitation, the general relativity theory has not been able to be formulated as a consistent gauge field theory. A unification of all four known forces remains so far to be only a dream.

Another flaw, the Higgs mechanism predicts a bosonic particle of unknown mass. Furthermore in perturbation theory the mass of this Higgs boson is affected by quadratic divergences. In order to get a proper electroweak symmetry breakdown for the SM, those divergences have to be physically cut at the TeV-scale, a hint for new physics around the TeV-scale. Without new physics between the EWK and the Planck scale one encounters the so-called hierarchy problem as the EWK scale is tiny compared to the Planck scale. One would need extraordinarily accurate parameters for evaluating the running mass from the Planck scale down to the EWK scale which cannot be done in a natural way in the SM. This is called the fine tuning problem.

Supersymmetry (SUSY) is a popular candidate theory to extend the SM and solve some of its most pronounced problems. Without going into details SUSY will shortly be explained following [19]. Other candidate theories solving those problems will not be mentioned. 
The theory formulated in the $1970 \mathrm{~s}^{3}$, connects fermions and bosons by a new introduced symmetry. Phenomenologically, every SM particle gets a supersymmetric partner different by a spin $1 / 2$. An operator $Q$ with the characteristics of an anticommuting spinor, allows transformations of fermions into bosons and vice versa.

$$
Q \mid \text { fermion }\rangle=\mid \text { boson }\rangle \quad \text { and } \quad Q \mid \text { boson }\rangle=\mid \text { fermion }\rangle
$$

SUSY connects masses and couplings of particles with different spin. This allows us to cancel the quadratic divergences of the radiative corrections of the Higgs mass by fermionic correction terms that introduce a negative factor due to fermion statistics. The fermionic character of the SUSY generator follows from the commutation rules for bosons and fermions. In a general form one can write a SUSY transformation as $\delta B=\epsilon \cdot f$, where $B$ stands for bosonic and $f$ for fermionic field. After using the commutation and anticommutation rules for the bosonic $[B, B]=0$ and fermionic fields $\{f, f\}=0$ one gets the infinitesimal transformation parameter $\{\epsilon, \epsilon\}=0$ which determines SUSY generators to be fermionic. The simplest representative is a two component Weyl spinor. The usual SUSY algebra can be written as

$$
\begin{gathered}
\left\{Q_{\alpha}, Q_{\beta}^{\dagger}\right\}=-2 \sigma_{\alpha \beta}^{\mu} P_{\mu} \\
\left\{Q_{\alpha}, Q_{\beta}\right\}=\left\{Q_{\alpha}^{\dagger}, Q_{\beta}^{\dagger}\right\}=0 \\
{\left[Q_{\alpha}, P^{\mu}\right]=\left[Q_{\alpha}^{\dagger}, P^{\mu}\right]=0}
\end{gathered}
$$

where one has the internal symmetry generators $Q_{\alpha}$ and the conjugated $Q_{\alpha}^{\dagger}$ as well as the 4-momentum operator $P^{\mu}$ and the spinoral indices $\alpha$ and $\beta$. One impressive result is that two SUSY transformations in succession lead to a translation in space-time as can be seen in the first of Equations (27). This opens a natural connection between SUSY and general relativity theory. SUSY includes the Poincare invariance as a gauge symmetry. For the particles and their superpartners the SUSY algebra has the following important corollaries. The supersymmetric particles should be degenerate in mass with their SM partners and have the same gauge quantum numbers. The particles are combined together with the superpartners in supermultiplets.

In contrast to the high energetic frontier of the SM one find serious design problems also in the low energetic region where the perturbative approach of the QCD breaks down. How can one explain low energetic phenomena of the strong interaction such as what is the proton, how is the quark-matter build and how one can describe it fundamentally? Those questions are not yet answerable. Attempts are made to

\footnotetext{
${ }^{3}$ For details about history and origin of SUSY refer to papers from Gol'fand and Likhtman, Akulov and Volkov and Wess and Zumino [20-22].
} 


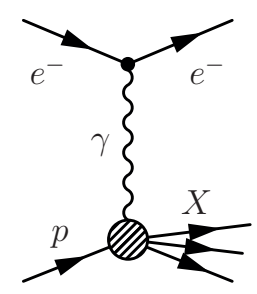

FiguRE 2.3. Feynman diagram for the lowest order inelastic scattering of electrons on protons for $-q^{2} \ll M_{Z}$. Note: the blob stands for the unknown proton vertex.

address these problems by non-perturbative approaches such as the Lattice QCD for example [23].

\section{Strong Interactions and Diffraction at High Energies}

The strong force and its dynamics at very high energies as introduced in the section above has been proven to be successfully described by the quantum chromodynamics theory (QCD). The coupling constant however is rising towards lower momentum transfer due to the non-abelian character of the $S U(3)_{c}$ based gauge field theory (see Figure 2.2). This leads to a breakdown of perturbative calculations of dynamical strong force processes towards lower momentum transfer. So far there is no real understanding in how to describe the proton, a hadronic object build out of quarks and gluons. Historically one knows that in analogy to the substructure of atoms found by inelastic scattering of charged particles, inelastically scattered protons gave strong hints that the proton is indeed not a fundamental point-like particle but has some substructure (see textbooks as [24] for deeper information about the structure of the proton). Experiments using high energetic electron beams on a proton target revealed a strong scaling violation. This opened the door to the rich substructure of the proton. In the highly inelastic collisions with an exchange of virtual photons, the proton will break up, $e p \rightarrow e X$, and complicate the measurements since the final state $X$ is not known (see Figure 2.3). The differential cross section for this inclusive process can be written by summing up all measurable states of $X$,

$$
\frac{d \sigma}{d E^{\prime} d \Omega}=\frac{\alpha^{2}}{q^{4}} \frac{E^{\prime}}{E} L^{\mu \nu} W_{\mu \nu}
$$

where $L^{\mu \nu}$ and $W_{\mu \nu}$ are second rank tensors describing the known electromagnetic subprocess $e^{-} \rightarrow e^{-}+\gamma$ and the complex and mostly unknown subprocess $p+\gamma \rightarrow X$ 
respectively. The latter can be written as,

$$
W^{\mu \nu}=\left(\frac{q^{\mu} q^{\nu}}{q^{2}}-g^{\mu \nu}\right) W_{1}+\frac{1}{M^{2}}\left(p^{\mu}-\frac{p \cdot q}{q^{2}} q^{\mu}\right)\left(p^{\nu}-\frac{p \cdot q}{q^{2}} q^{\nu}\right) W_{2}
$$

with $W_{1,2}\left(q^{2}, x\right)$ being so-called (inelastic) structure functions depending on two independent variables $q^{2}$ and $x=\frac{-q^{2}}{2 p \cdot q}$. $E$ and $E^{\prime}$ in Equation (28) are the energies of the incoming and outgoing electrons respectively. The inelastic structure functions show no strong dependence on $q^{2}$ at higher energies but on the dimensionless variable $x$, called Bjorken $x$.

$$
\begin{aligned}
M W_{1}\left(q^{2}, x\right) & \rightarrow F_{1}(x), \\
\frac{-g^{2}}{M x} W_{2}\left(q^{2}, x\right) & \rightarrow F_{2}(x)
\end{aligned}
$$

This phenomenon, known as Bjorken scaling [25], is a sign that the virtual photon in the scattering process is interacting with point-like particles without dependency on a mass scale $\left(q^{2}\right)$ as in elastic scattering on a non point-like particle. Introducing the proton consisting of point-like particles (partons), $W_{1,2}$ can be expressed in terms of the partons charge $Q$, momentum $p_{i}$ and the momentum of the virtual photon $q$. The parton momentum hereby is a fraction of the total proton momentum $p_{i}=\kappa_{i} \cdot p$. One finds the relations,

$$
\begin{aligned}
& F_{1}(x)=\frac{1}{2 x} F_{2}(x) \\
& F_{2}(x)=\sum_{i} Q_{i}^{2} x f_{i}
\end{aligned}
$$

with $f_{i}$ being the probability that the parton has a certain fraction of the momentum of the proton. Now, Equation (32) known as the Callan-Gross relation indicates that the partons are spin 1/2 particles and Equation (33) shows the scaling behavior at high $q^{2}$ and the importance of the parton probability density functions $f_{i}$ for further understanding of the inner structure of the proton. As it turns out, the proton does not consist only of charged partons (quarks) but also of uncharged partons, the gluons. A reasonable model describes the proton as a structure of three valence quarks ( $u-u-d$ quark configuration) all bound together by gluons plus an additional undefined number of fluctuating so-called sea-quark pairs. All constituents are described by their own structure function.

Especially for accelerator experiments with colliding hadrons, it is very important to know the probability of partons to be found within a hadron carrying a certain momentum fraction of the hadrons momentum. These are presented in the parton density functions or short PDF that cannot be obtained by perturbative QCD calculations and have to be determined via experiments using data mostly from deep 

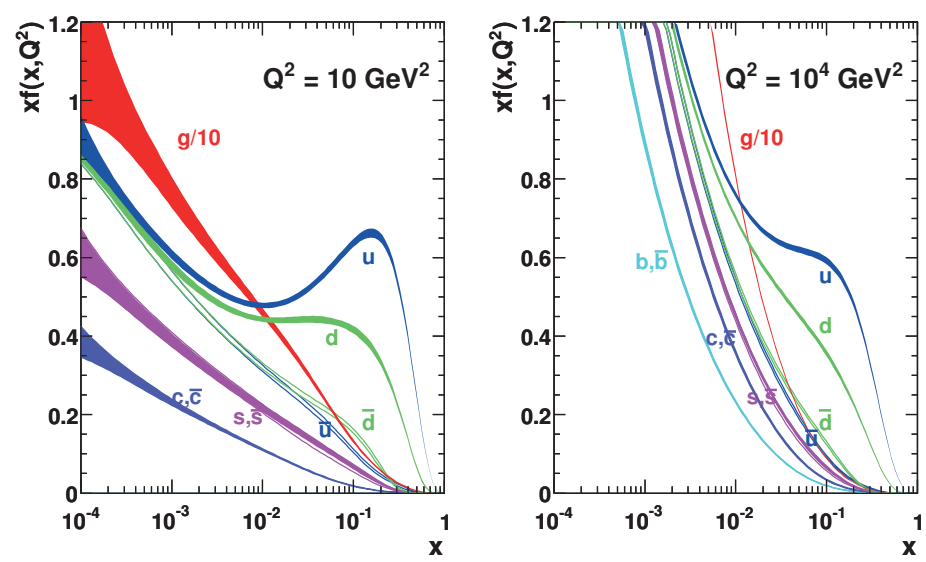

Figure 2.4. Parton distribution function (MSTW2008) in the next to leading order version for two different energy scales. The error bands represent the $68 \%$ C.L. Note the increasing uncertainties for lower $Q^{2}\left(=-q^{2}\right)$. Plot taken from the website of the authors of $[\mathbf{2 6}]$.

inelastic scattering down to low $Q^{2}\left(=-q^{2}\right)$ where perturbative calculations are still valid. The parametrization depending on $x$ is scaled up to higher energy scales using DGLAP evolution equations. Extensive fitting to present data is done to obtain the input parameters. An example PDF of the MSTW collaboration is shown in Figure 2.4 obtained in a next to leading order (NLO) global analysis. DGLAP equations (Dokshitzer-Gribov-Lipatov-Altarelli-Parisi [27-29]) are a set of integro-differential equations resuming effective parton densities that have a dependency on the scale $Q^{2}$. In fact, Bjorken scaling is slightly violated due to real gluon emission that adds a logarithmic scale factor to the structure functions. This is a complication towards a real model of the proton structure compared to the simple parton model mentioned above. Adding the gluon emission corrections to the photon-parton scattering one obtains a new version of the structure function Equation (33),

$$
F_{2}\left(x, q^{2}\right)=\sum_{q_{i}} Q_{i}^{2} x\left(q_{i}(x)+\frac{\alpha_{s}\left(q^{2}\right)}{2 \pi} \ln \left(\frac{-q^{2}}{\mu^{2}} \int_{x}^{1} \frac{d y}{y} q_{i}(y) P_{q_{i} q_{i}}\left(\frac{x}{y}\right)\right)\right)
$$

where

$$
P_{q_{i} q_{i}}(z)=\frac{4}{3}\left(\frac{1+z^{2}}{1-z}\right)
$$

is the quark $\left(q_{i}\right)$ splitting function with $z$ being the fraction of the momentum the quarks momentum is reduced due to gluon emission. Here $z=x / y$ where $y$ is the fraction of 
the proton momentum the parton holds. To get hold on the infrared divergence, a cutoff factor $\mu^{2}$ is introduced. The second term of Equation (34) containing the scale dependency can be written as a evolution equation,

$$
q^{2} \frac{d}{d q^{2}}\left(\begin{array}{c}
q_{i}\left(x, q^{2}\right) \\
g\left(x, q^{2}\right)
\end{array}\right)=\frac{\alpha_{s}\left(q^{2}\right)}{2 \pi} \int_{x}^{1} \frac{d y}{y}\left(\begin{array}{cc}
P_{q_{1} q_{1}}^{+}\left(\frac{x}{y}\right) & P_{q_{i} g}\left(\frac{x}{y}\right) \\
P_{g} q_{i}\left(\frac{x}{y}\right) & P_{g g}\left(\frac{x}{y}\right)
\end{array}\right)\left(\begin{array}{c}
q_{i}\left(y, q^{2}\right) \\
g\left(y, q^{2}\right)
\end{array}\right)
$$

where also other splitting functions are added that contribute to the quark densities such as gluon pair or quark anti-guark pair splitting. This last equation stands for what is known as the DGLAP equations. These equations enable us to predict how the distribution function changes logarithmically over the energy scale, and are the basis for producing the PDF's as shown in Figure 2.4.

Before approaching strong interaction physics and diffraction at small 4-momentum transfer or long distances one should emphasize the importance of the QCD factorization theorem that enables us to compute particle processes at least partly using perturbative techniques. The factorization theorem allows us to separate a short distance process such as the production of, for example, a dijet system in hadron-hadron collisions from the underlying long distance processes described by the universal parton densities. Such a cross section can be calculated as,

$$
\sigma=\sum_{i, j} \int d x_{1} d x_{2} f_{i}\left(x_{1}, \mu^{2}\right) f_{j}\left(x_{2}, \mu^{2}\right) \hat{\sigma}_{i, j}\left(x_{1} p_{1}, x_{2} p_{2}, \alpha_{s}\left(\mu^{2}\right)\right),
$$

where $f_{i}$ represent the universal parton densities that can be determined by global fits to experimental data as mentioned above, and $\hat{\sigma}_{i, i}$ denotes the hard perturbative calculable parton scattering production process of the dijet system. The factorization scale $\mu$ is usually set to the QCD renormalization scale. The factorization theorem will be of great importance predicting hard-diffractive processes such as central exclusive production of dijets or diphotons, the key-process of this thesis. What exactly diffraction is, especially in the context of high energy physics remains to be discussed in the next section.

\subsection{What is Diffraction?}

A large contingent of particle dynamics is represented by diffractive processes. Those are inelastic processes somewhat closer to elastic scattering. The total diffractive cross section is close to half of the total cross section. The name diffraction came historically from the field of optics. Light scattering at an object or passing through an slit with dimensions close to its wavelength shows some diffractive pattern. Such effects have been observed as well in nuclear and hadronic scattering processes. In particle physics however the analogy to the diffraction in optics is very limited. This 
opens up the question, how should one define diffraction in high energy particle physics that turns out to make up roughly $40 \%$ of the total cross section in proton (anti)proton collisions?

A more modern definition of diffraction defines diffractive processes as interactions with vacuum quantum number exchange or color-singlet exchange ${ }^{4}$. Or in terms of the s-channel unitarity, diffraction is scattering on a target that absorbs coherent states that build up the incoming hadrons. In the t-channel Pomeron picture, the vacuum quantum number or colorless exchange usually involves large rapidity gaps ${ }^{5}$ as an observable which is often used to define diffractive physics.

We divide diffraction into soft and hard. Soft diffraction includes physics with low 4-momentum transfer or larger distances where perturbative QCD is not applicable and only some phenomenological models are able to describe experimental results. Some success has been achieved with models from Good and Walker [35], Miettinen and Pumplin [36] and Regge [42]. Hard diffraction stands for all those diffractive processes that include higher 4-momentum transfer covering short distances, where perturbative QCD (pQCD) is at least to some extent applicable.

We can classify diffractive processes experimentally at hadron-hadron colliders. As sketched in Figure 2.5 diffractive processes can be divided into elastic scattering of hadrons, single diffraction, double diffraction and double pomeron exchange processes. A single diffractive process consists of a hadron that survives the collision intact and a dissociated system in the other hemisphere with a void of particles (rapidity gap) between. One speaks about double diffraction when both hadrons do not survive the collision, fall apart and fragment to whatever the phase-space allows. Between the dissociated systems one finds most likely a rapidity gap. A somewhat more complicated process, the double pomeron exchange (DIPE) ${ }^{6}$ has usually a signature of two outgoing and surviving ${ }^{7}$ hadrons with an additional centrally produced system $X$. A subset of DIPE is called central exclusive production (CEP) in case all the energy

\footnotetext{
${ }^{4}$ Color-singlet means a colorless state with zero color quantum numbers, invariant under $S U(3)$ color transformations and yield zero under color ladder operators. The color-singlet expressed out of the three color charges is written as $1 / \sqrt{3}(r \bar{r}+g \bar{g}+b \bar{b})$. Compare with two spin $1 / 2$ particles with $\left|S^{2}, S_{z}\right\rangle$. Here $|0,0\rangle=1 / \sqrt{2}(\uparrow \downarrow-\downarrow \uparrow)$ corresponds to the spin 0 singlet state.

${ }^{5}$ Under rapidity gap one understands experimentally a void of particles within a rapidity region. More about rapidity and rapidity gaps can be found in Appendix 2.

${ }^{6}$ To be more precise, DIPE is used mostly due to the fact that the underlying object that is exchanged is still not fully known. The name Pomeron was used before the advent of the QCD to describe such an object with vacuum quantum numbers, and still is, especially for soft diffractive processes. For hard scale diffraction the description of the exchanged object as QCD multi-gluon ladders has some success. Therefore, more modern is the usage of the unambiguous phrase CEP.

${ }^{7}$ It has to be mentioned that DIPE processes also include cases when one or two hadrons break up, which is not shown in Figure 2.5(e).
} 


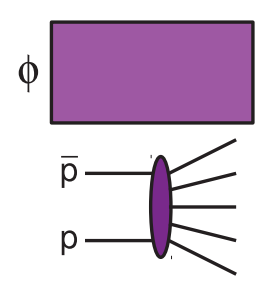

a) non-diffractive processs
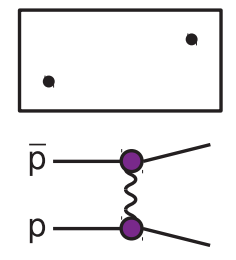

b) elastic scattering

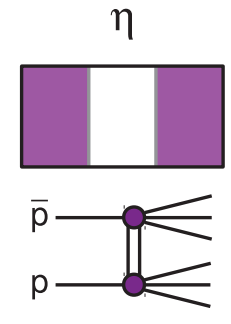

c) double diffraction
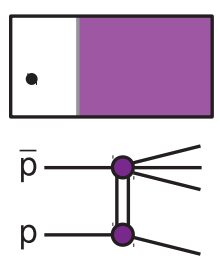

d) single diffraction
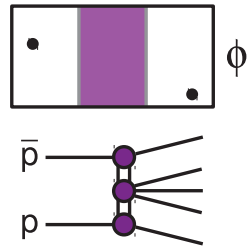

e) double pomeron exchange

Figure 2.5. Processes of hadron-hadron collision events. The black dots in the $\eta-\phi$ space denote outgoing surviving hadrons. The shaded (pink) area denotes activity in form of outgoing particles whereas rapidity gaps are shown as white areas.

lost by the protons is used to build up the system $X$ in a hard-scattering subprocess. One finds rapidity gaps on each side of the central system. CEP includes not only strongly interacting processes but also purely QED mediated processes or production via pomeron photon fusion. The latter is called photo-production.

To have more technical information about diffraction, one should first mention somewhat general and rather important concepts and theorems. Historically they have been part of the approach to understand the high energy behavior of hadronic interactions including total cross section and dynamics of scattering processes, elastic and diffractive.

Some analogy can be found in the Fraunhofer diffraction in the context of optics. The cross sections for non-relativistic scattering of a plane wave off a potential obtained using the eikonal approximation is written as,

$$
\begin{aligned}
\sigma_{\mathrm{el}} & =\int d^{2} \boldsymbol{b}|\Gamma(b)|^{2} \\
\sigma_{\text {inel }} & =\int d^{2} \boldsymbol{b}\left[2 \operatorname{Re} \Gamma(b)-|\Gamma(b)|^{2}\right] \\
\sigma_{\text {tot }} & =\int d^{2} \boldsymbol{b} 2 \operatorname{Re} \Gamma(b)
\end{aligned}
$$

Here $\Gamma(b)$ represents the profile function, the inverse Fourier transform of the scattering amplitude and $\boldsymbol{b}$ the impact parameter.

In the relativistic case one can use the $S$-matrix formalism, where $S$ is an operator transforming a state $|i\rangle$ at the time $-\infty$ into a state $|f\rangle$ at the time $+\infty,|f\rangle \rightarrow S|i\rangle$. One of the properties of $S$ is unitarity, $S^{\dagger} S=S S^{\dagger}=\mathbb{1}$, that is directly related to the conservation of the transition probability of an initial state $|i\rangle$ to any arbitrary 


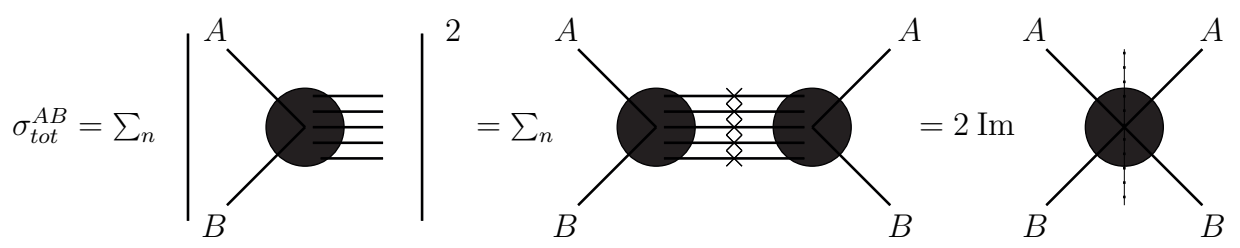

FiguRE 2.6. Graphical presentation of the optical theorem.

state $|k\rangle$. Integrating over all possible final states will give the probability of 1 .

$$
\sum_{k} P(i \rightarrow k)=\sum_{k}|\langle k|S| i\rangle|^{2}=\sum_{k}\left\langle i\left|S^{\dagger}\right| k\right\rangle\langle k|S| i\rangle=\left\langle i\left|S^{\dagger} S\right| i\right\rangle=1
$$

In terms of transition matrix $T, S=\mathbb{1}+i T$ one gets,

$$
\left(\mathbb{1}-i T^{\dagger}\right)(\mathbb{1}+i T)=\mathbb{1} \Rightarrow i\left(T^{\dagger}-T\right)=T^{\dagger} T
$$

Now using this matrix element for the transition of initial to final state and inserting a complete set of arbitrary states,

$$
i\left\langle f\left|T^{\dagger}-T\right| i\right\rangle=\sum_{n}\left\langle f\left|T^{\dagger}\right| n\right\rangle\langle n|T| i\rangle
$$

we get

$$
2 \operatorname{Im} T_{i f}=\sum_{n} T_{f n}^{*} T_{i n}
$$

If one takes initial and final state as identical $(|f\rangle=|i\rangle)$ as in forward elastic scattering we get the optical theorem,

$$
2 \operatorname{Im} T_{\mathrm{el}}(s, t=0)=\sum_{n}|T(i \rightarrow n)|^{2}=\sigma_{\mathrm{tot}}
$$

Figure 2.6 shows a graphical presentation of the optical theorem. The sum over all inelastic and elastic channels is connected to the imaginary part of the elastic scattering amplitude, meaning virtually $A+B$ goes to anything and anything goes to $A+B$. The crosses in the central diagram denote the on-mass shell summation and the dashed line in the last diagram denotes the discontinuity of the amplitude. The optical theorem is practically used to determine the total cross section at hadron colliders ${ }^{8}$ by measuring the forward elastic amplitude at $t=0$.

${ }^{8}$ The TOTEM experiment at the LHC uses a modified form of the optical theorem that links the rate of the elastic and inelastic events in the forward region to the total cross section [30] by,

$$
\sigma_{\text {tot }}=\frac{16 \pi}{\left(1+\rho^{2}\right)} \frac{\left(d N_{\text {elastic }} / d t\right)_{t=0}}{N_{\text {elastic }}+N_{\text {inelastic }}} \text { or } \sigma_{\text {tot }}^{2}=\left.\frac{16 \pi}{\left(1+\rho^{2}\right)} \frac{\mathrm{d} \sigma_{\text {elastic }}}{\mathrm{d} t}\right|_{t=0} .
$$

Note: The second formula is not luminosity independent; $\rho=\operatorname{Re}\left[f_{\mathrm{el}}(0)\right] / \operatorname{Im}\left[f_{\mathrm{el}}(0)\right]$ is the forward nuclear amplitude. 
A fundamental key-point to the phenomenological approach to low-x physics is the analyticity of scattering amplitudes. The amplitudes depend on kinematic variables, usually the Mandelstam variables $s$ and $t$ (see Appendix A). It can be shown that the amplitudes for different kinematical channels are given by the same function. The feature to continue the amplitudes analytically to other kinematical channels is known as crossing.

Utilizing the partial wave expansion of the amplitudes in form of the FroissartGribov formulation one can show that the total cross section does not grow faster than the squared logarithm of the collision energy,

$$
\sigma_{\text {tot }}(s) \underset{s \rightarrow \infty}{\leq} \text { const } \times \ln ^{2}\left(s / s_{0}\right)
$$

This known as the Froissart or Froissart-Martin bound where $s$ is the square of the center of mass energy in $\mathrm{GeV}^{2}, s_{0} \sim 1 \mathrm{GeV}^{2}$ and the const $=\pi / m_{\pi}^{2} \approx 60 \mathrm{mb}[\mathbf{3 1}, \mathbf{3 2}]$.

Pomeranchuck postulated and proved a theorem stating that at high enough energies the total cross section of proton-proton and proton-antiproton scattering becomes equal. This has been seen at collider experiments not only for proton-(anti)proton but also for example in proton-pion scattering $\left(p \pi^{+}\right.$versus $\left.p \pi^{-}\right)$. The total cross section for proton-(anti)proton scattering versus the center of mass collision energy is shown in Figure 2.7. Easily one can see the asymptotic behavior of the proton-proton and proton-antiproton total cross section towards higher energies as well as the overall growth being below the Froissart-(Martin) bound.

A description of diffractive scattering in the impact parameter $b$ space using the s-matrix formulation and partial wave expansion, can be formulated. One assumes that $b$ is "frozen" in high energy interactions. Using the optical theorem thanks to unitarity,

$$
2 \operatorname{Im} T_{\mathrm{el}}(s, b)=\left|T_{\mathrm{el}}(s, b)\right|^{2}+\sigma_{\text {inel }}(s, b)
$$

we find,

$$
\begin{aligned}
\sigma_{\mathrm{el}} & =\int d^{2} b\left|T_{\mathrm{el}}(s, b)\right|^{2}=\int d^{2} b\left(1-\exp \left(-\frac{\Omega}{2}\right)\right)^{2} \\
\sigma_{\text {inel }} & =\int d^{2} b\left[2 \operatorname{Im} T_{\mathrm{el}}(s, b)-\left|T_{\mathrm{el}}(s, b)\right|^{2}\right]=\int d^{2} b(1-\exp (-\Omega)) \\
\sigma_{\text {tot }} & =\int d^{2} b 2 \operatorname{Im} T_{\mathrm{el}}(s, b)=\int d^{2} b 2\left(1-\exp \left(-\frac{\Omega}{2}\right)\right) .
\end{aligned}
$$

At high energies the amplitude is expected to be purely imaginary. The right-hand side of the equations is written in the eikonal parametrization with $T_{\mathrm{el}}(s, b)=i(1-\exp (-\Omega / 2))$, where $\Omega$ is the opacity. From the equations one can read that at high energies with $\Omega \gg 1$ the inelastic cross section dominates. In case of 


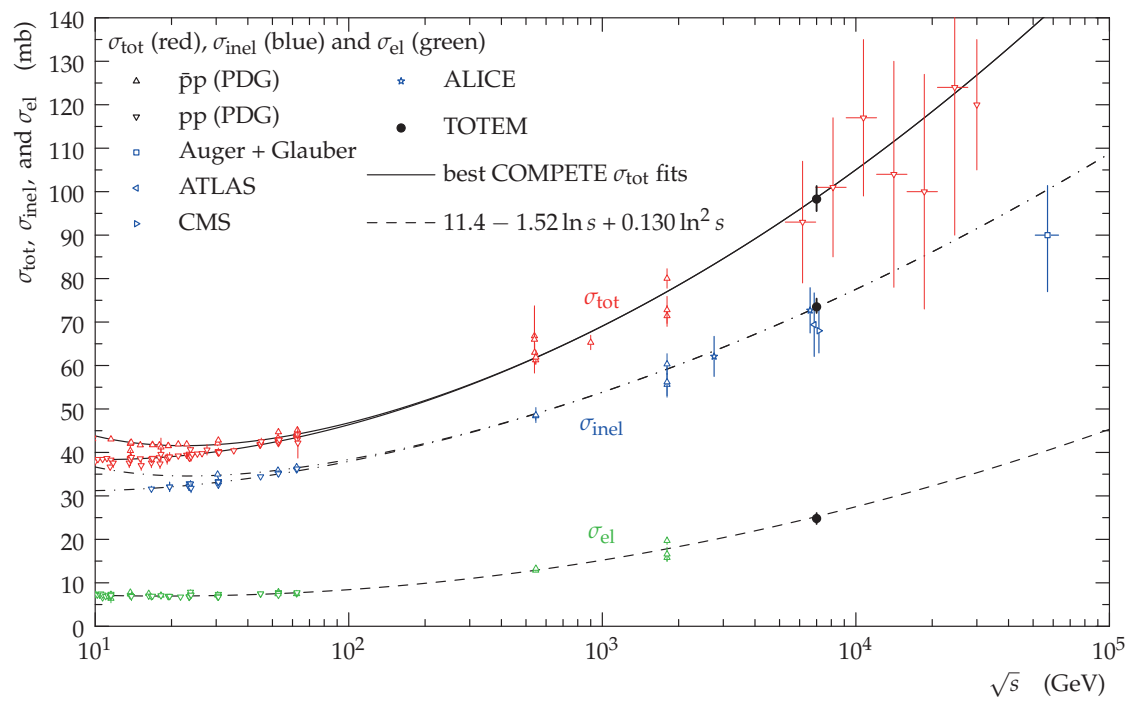

Figure 2.7. Total cross section versus $\sqrt{s}$ for proton-(anti)proton scattering measured at various collider experiments. The plot is taken from [30] including the latest measurements at the LHC energy of $7 \mathrm{TeV}$. Elastic and inelastic cross section are shown as well.

a black disc with radius $R, \Omega=\infty$ for $b<R$ otherwise 0 . In terms of cross section one finds $\sigma_{\text {inel }}=\pi R^{2}$ representing the total absorption, which is the area of the black disc. The elastic cross section is $\sigma_{\mathrm{el}}=\pi R^{2}$. The sum of the elastic and inelastic cross section gives a total cross section of $\sigma_{\text {tot }}=2 \pi R^{2}$. At the LHC towards small $b$ one might close up to the black disc limit. However, it is not clear whether it will be reached or it lays beyond the reach of the LHC. This topic is discussed e.g. in $[33,34]$ and references therein.

Up to now mostly topics around elastic diffraction have been discussed. As already said, inelastic processes dominate at high energies. What are inelastic diffractive processes and how can one describe those? One early approach to describe inelastic diffraction in the unitary s-channel picture was shown by Good and Walker.

\subsection{Soft Diffraction}

2.2.1. Good and Walker Approach. The Good-Walker formalism is the first attempt to describe soft diffraction from a quantum mechanical point of view. For Good and Walker the hadron is an composite object with internal structure of unknown coherent fluctuating constituents which are frozen at high energy collisions and 
separated by large distances [35]. In inelastic diffraction constituents of the hadron scatter and destroy the coherence, leading to excited states or production of new hadrons. Miettinen and Pumplin [36] took over that picture and introduce so-called wee-partons as diffractive eigenstates of the hadrons with a Poissonian character. The incident hadron is a linear combination of diffractive eigenstates,

$$
|B\rangle=\sum_{k} C_{k}\left|\psi_{k}\right\rangle
$$

Those diffractive states are eigenstates of the scattering operator,

$$
\operatorname{Im} T\left|\psi_{k}\right\rangle=t_{k}\left|\psi_{k}\right\rangle
$$

where $t_{k}$ are the eigenvalues depending on the impact parameter $b$. In an inelastic diffractive scattering process the incident state $|B\rangle$ will not survive. Its eigenstates are absorbed by the collision partner. However, the outgoing particles (depending on the phase space) as a whole will have the same quantum numbers as the incident hadron. With $\langle B \mid B\rangle$ normalized to 1, the imaginary part of the transition operator will give us the elastic and total cross sections (optical theorem),

$$
\langle B|\operatorname{Im} T| B\rangle=\sum_{k}\left|C_{k}\right|^{2} t_{k} \equiv\langle t\rangle
$$

Thus

$$
\begin{aligned}
\frac{d \sigma_{\mathrm{el}}}{d^{2} \boldsymbol{b}} & =\langle t\rangle^{2} \\
\frac{d \sigma_{\mathrm{tot}}}{d^{2} \boldsymbol{b}} & =2\langle t\rangle \\
\frac{d \sigma_{\text {inel }}}{d^{2} \boldsymbol{b}} & =\left\langle t^{2}\right\rangle-\langle t\rangle^{2}
\end{aligned}
$$

where the inelastic cross section can be obtained by removing the elastic part of the total cross section. Miettinen and Pumplin took the diffractive eigenstates as $N$ independent wee partons within the hadron as function of rapidity $y$ and the impact parameter vector $\boldsymbol{b}$,

$$
\left|\psi_{k}\right\rangle \equiv\left|\boldsymbol{b}_{1}, \ldots, \boldsymbol{b}_{N}, y_{1}, \ldots, y_{N}\right\rangle
$$

Inserting the new defined diffractive eigenstates into Equation (51) and assuming independent interaction between the wee partons following the Poisson statistics one can define the probability $\left|C_{N}\left(\boldsymbol{b}_{i}, y_{i}\right)\right|^{2}$ and thus gain the expressions for the cross 
sections as shown in [37].

$$
\begin{aligned}
\frac{d \sigma_{\text {tot }}}{d^{2} \boldsymbol{b}} & =2\left(1-\exp \left(-\frac{4}{9} G^{2} e^{-\frac{1}{3} \cdot \frac{b 2}{\beta}}\right)\right) \\
\frac{d \sigma_{\mathrm{el}}}{d^{2} \boldsymbol{b}} & =\left(1-\exp \left(-\frac{4}{9} G^{2} e^{-\frac{1}{3} \cdot \frac{b 2}{\beta}}\right)\right)^{2} \\
\frac{d \sigma_{\text {diff }}}{d^{2} \boldsymbol{b}} & =\exp \left(-\frac{8}{9} G^{2} e^{-\frac{1}{3} \cdot \frac{b^{2}}{\beta}}\right)\left(\exp \left(\frac{1}{9} G^{2} e^{-\frac{1}{2} \cdot \frac{b^{2}}{\beta}}\right)-1\right)
\end{aligned}
$$

The two parameters $G^{2}$ and $\beta$ that the model depends on, can be experimentally obtained from elastic and total cross section measurements. The authors of reference [37] could successfully describe the low-mass diffractive dissociation in hadron hadron collisions at Tevatron energies using this model.

To be more comprehensive, already Feynman and Gribov introduced a model to approach soft diffraction, a pre-QCD parton model [38-40]. The hadron constituents are called partons and have no quantum numbers. In a space-time evolution picture, before a hadron collision those point-like partons are in a coherent state, resulting from fluctuations at high energies with a long lifetime of the order of $E / \mu^{2}$. Partons can itself create new partons that lead to a cascade of $N$ soft partons before a collision with a target. In fact, those soft partons with small $x$, called wee-partons, dominate the interaction with the target at small 4-momentum transfer. The distribution of the partons inside the hadrons can be called homogeneously and interactions between wee-partons can be seen as universal, independent of the type of hadron.

However, high-mass diffraction is not well described by the above s-channel approaches $^{9}$. This issue will be addressed later. First the t-channel approach, a somewhat competing description of diffraction, will be explained.

2.2.2. Regge Theory and the Pomeron. Historically connected to the tchannel picture is the phenomenological Regge theory (see [42-44] and references therein). Regge, who had the idea to continue angular momenta to complex numbers in describing non-relativistic quantum scattering at a potential. Based on unitarity, analyticity and crossing he initiated a theory that could describe hadronic resonances. A simple relation between the hadronic masses and their angular momenta was found (see Figure 2.8). At the time it looked very promising to compete against the still premature quantum field theory.

\footnotetext{
${ }^{9}$ This is not totally true as e.g. studies by the Tel Aviv group show. They use a new approach to describe soft and hard diffraction combined within a $N=4$ SYM model. The diffractive processes within this theory are described by the Good Walker mechanism using the two-channel model [41]. Their model describes cross section measurements including the new TOTEM data sufficiently well over a large energy range which gives confidence to their approach.
} 


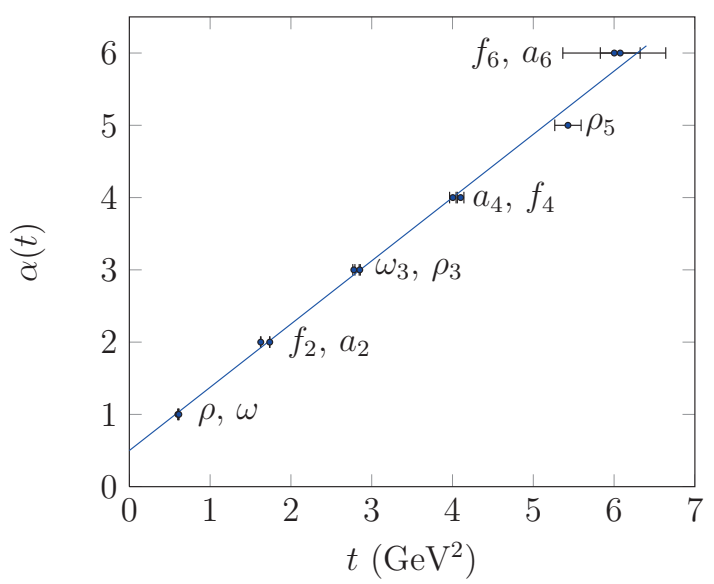

Figure 2.8. Mesonic Regge trajectories. Trajectories for various mesons with different spin are superimposed. Note that the $\mathrm{x}$-axis represents the masses of the mesons $M^{2}$ as we are in the s-channel.

To get a more detailed view of this approach to hadronic scattering one starts from the partial wave expansion of the scattering amplitude following loosely [45]. Due to analyticity and crossing the s-channel amplitude and the t-channel amplitude are connected and can be switched by exchanging the Mandelstam variables. Sommerfeld and Watson showed how to express the scattering amplitude as an integral over the angular momentum in the whole complex plane.

$$
A(s, t)=\frac{1}{2 i} \oint_{C} d l(2 l+1) \frac{a_{l}(t)}{\sin \pi l} P_{l}(\cos \theta)
$$

where $\cos \theta=1+2 s / t, P_{l}$ is derived from Legendre polynomials and $a_{l}$ are partial wave amplitudes. It turns out that one needs to correct the above function to overcome a divergence problem for $l \rightarrow \infty$ and keep the amplitudes continuous in the complex plane. Two alternating functions are added $a_{l}^{( \pm)}(t)$ for even and odd angular momentum that are analytic continuations of the partial wave amplitudes. One gets

$$
A(s, t)=\frac{1}{2 i} \oint_{C} d l(2 l+1) \frac{P_{l}(\cos \theta)}{\sin \pi l}\left(\frac{e^{-i \pi l}+1}{2} a_{l}^{(+)}(t)+\frac{e^{-i \pi l}-1}{2} a_{l}^{(-)}(t)\right)
$$

where the coefficients $(\exp (-i \pi l) \pm 1) / 2$ are called signature factors. Making use of a smart integration contour change and taking care of poles and cuts in the complex plane that are affected, one can show that for high energies with $s \gg|t|$ only the poles 


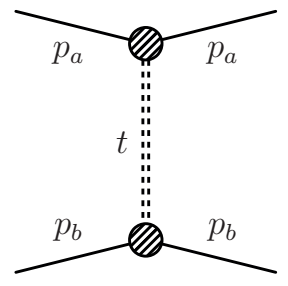

FiguRE 2.9. Diagram of elastic scattering of hadrons via t-channel "reggeon" exchange.

and cuts contribute to the scattering amplitude, that now reads

$$
A(s, t) \underset{\substack{s \\ s \gg|t|}}{\approx} \frac{1}{2}\left(e^{-\pi \alpha(t)} \pm 1\right) \tau(t) s^{\alpha(t)} .
$$

Here $\tau(t)$ is a function of the residue of the leading Regge pole $\alpha(t)$. Factorization allows us to see this scattering amplitude as a result of a reggeon exchange between two particles $p_{a}, p_{b}$ as illustrated in Figure 2.9 (note: inelastic case is not shown here but should be included, $\left.p_{a} p_{b} \rightarrow p_{c} p_{d}\right)$. Factorized the amplitude can be written as

$$
A(s, t) \underset{\substack{c \rightarrow \infty \\ s \gg|t|}}{\approx} \frac{\left(e^{-\pi \alpha(t)} \pm 1\right)}{2 \sin \pi \alpha(t)} \frac{\beta_{p_{a c}}(t) \beta_{p_{b d}}(t)}{\Gamma(\alpha(t))} s^{\alpha(t)},
$$

where $\beta_{p_{a c}}(t), \beta_{p_{b d}}(t)$, factorized from the Regge pole residue $\tau(t)$, describe the coupling of the hadron with the reggeon and $\Gamma(\alpha(t))$ removes unphysical poles at negative spin values. The reggeon itself is not a particle as the angular momenta $t$ are not discrete. However at integer or half-integer values the pole resonances can be seen as exchange of physical particles. Plotting the spin over the masses $\left(M^{2}\right)$ of meson particles will show a linear dependence, as shown Figure 2.8. Thus for small t one can write

$$
\alpha(t)=\alpha(0)+\alpha^{\prime} t
$$

where the intercept $\alpha(0) \approx 0.5$ and the slope $\alpha^{\prime} \approx 0.9 \mathrm{GeV}^{-2}$ obtained from a fit to the data of the mesonic plot (Figure 2.8). In this plot all leading Regge trajectories for the various mesons are superimposed. Remarkably is that the linear behavior of the Regge trajectories continues into the negative side of $t$. Here one looks at the scattering of $\pi^{-} p \rightarrow \pi^{0} n$ which has isospin $I=1 t$-channel exchange comparable to the parity even mesons with isospin $I=1$. The $\alpha(t)$ values obtained from data lay on the same interpolated linear curve over some mass interval till the point when $\alpha(t)$ goes through zero representing an unphysical pole. This could explain the dip in the cross section curve of this process at $t \sim-0.6 \mathrm{GeV}^{2}$.

In general it was found that the Regge-pomeron formalism shows sufficient success in describing elastic hadron-hadron collisions. From early measurements it was known 
that the total cross section is falling for moderate high energies until it rises slowly again with growing $s$ staying well below the Froissart-(Martin) bound. Contributions from reggeon exchange with mesonic quantum numbers always result in a decreasing cross section, having an intercept $\alpha(0)<1$. A rising cross section will favor a dominating exchange of an object with quantum numbers of the vacuum and an intercept slightly larger than 1. As well known (see e.g. [4]), the elastic amplitude for the reaction $p_{a} p_{b} \rightarrow p_{a} p_{b}$, that is shown in Figure 2.9 in form of a t-channel reggeon exchange, can be written

$$
\frac{A(s, t)}{s}=\beta_{p_{a}}(t) \beta_{p_{b}}(t) \omega(t)\left(\frac{s}{s_{0}}\right)^{\alpha_{\mathbf{P}}(t)-1},
$$

with $\alpha_{\mathbb{P}}(t)$ being now called pomeron trajectory for an object with vacuum quantum numbers. The functions $\beta_{p_{a}}(t), \beta_{p_{b}}(t)$ describe the pomeron hadron vertex, $\omega(t)$ is known as the signature factor in a modified form,

$$
\omega(t)=i-\cot \left(\frac{\pi \alpha_{\mathbb{P}}(t)}{2}\right),
$$

and $s_{0}$ is an arbitrary scaling constant. Using the optical theorem (see Equation (45)) that links the total cross section to the forward part of the elastic amplitude at $t=0$, $\operatorname{Im} A(s, 0)=s \sigma_{\text {tot }}(s)$, one gets

$$
\sigma_{\text {tot }}(s)=\beta_{p_{a}}(0) \beta_{p_{b}}(0)\left(\frac{s}{s_{0}}\right)^{\alpha_{\mathbf{P}}(0)-1} .
$$

As the total cross section is raising with energy as shown by experiments (see Figure 2.7), one finds a reasonable good description of the data if the pomeron trajectory has an intercept at $t=0, \alpha_{\mathbb{P}}>1$. In fact fits to data-analysis results yield an pomeron trajectory, parametrized as a first order polynomial, of

$$
\alpha_{\mathbb{P}}(t) \approx \alpha_{\mathbb{P}}(0)+\alpha_{\mathbb{P}}^{\prime} t \approx 1.08+\left(0.25 \mathrm{GeV}^{-2}\right) t
$$

For mesonic trajectories (as seen e.g. in Figure 2.8) one has an intercept of $\approx 0.5 \mathrm{GeV}$ which suggest a decreasing cross section. For higher energies those contributions can be neglected. Newer fits suggest an even higher intercept $\alpha_{\mathbb{P}}(0)$ and promote a contribution of a hard pomeron exchange to the overall result. The pomeron which was named after Pomeranchuck is in fact not a real particle but can be seen as an multi-gluon exchange or glueball exchange towards small $t$. A relative simple relation that is based entirely on the Regge model, provides a surprisingly good description of the total cross section up to Tevatron energies [46]. The equations for proton-(anti)proton scattering, 
(a)

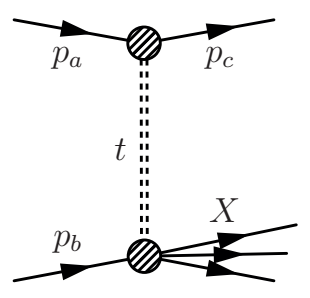

(b)

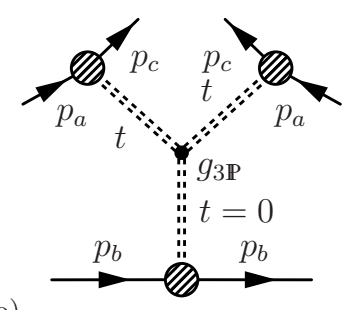

Figure 2.10. (a) Feynman diagram of single diffractive dissociation in hadron hadron collisions. (b) Triple pomeron version according to Al. Mueller.

depend just on a small number of parameters fitted to data,

$$
\begin{aligned}
\sigma_{\text {tot }}^{p \bar{p}} & =21.70 s^{0.0808}+98.39 s^{-0.4525} \\
\sigma_{\text {tot }}^{p p} & =21.70 s^{0.0808}+56.08 s^{-0.4525},
\end{aligned}
$$

where the first term represents the pomeron exchange, identical for $p p$ and $p \bar{p}$ collision, and the second term represents the exchange of the $\rho, \omega, f$, and $a$ mesons. The couplings of the mesons to protons and antiprotons are different resulting in the different slopes as shown in Figure 2.7.

The Regge formalism also provides a way to express single and double diffraction at small $t$ exchange to some extent. Single diffractive reaction $p_{a}+p_{b} \rightarrow p_{a}+X$, as shown in Figure 2.10 on the left, has one hadron dissociating into a system $X$. The other hadron survives the collision. Here one can make use of the Regge-Mueller formalism. Of central importance is the extension of the optical theorem by Mueller which links the inclusive cross section of the reaction $p_{a}+p_{b} \rightarrow p_{c}+X$ (see Figure 2.10(a)) to the forward elastic amplitude of a three body process $p_{a}+p_{b}+\bar{p}_{c} \rightarrow p_{a}+p_{b}+\bar{p}_{c}$ (see Figure 2.10(b)),

$$
s \frac{d^{2} \sigma^{\left(p_{a}+p_{b} \rightarrow p_{c}+X\right)}}{d M_{X}^{2} d t} \sim \frac{1}{s} \operatorname{Disc}_{M_{X}^{2}} A\left(p_{a}+p_{b}+\bar{p}_{c} \rightarrow p_{a}+p_{b}+\bar{p}_{c}\right),
$$

where $\operatorname{Disc}_{M_{X}^{2}}$ refers to the discontinuity in the $M_{X}^{2}$ cut of the elastic amplitude [47]. In combination with the Regge formalism the right-hand side of Equation (72) can be written for $s, M_{X}^{2} \rightarrow \infty$ and $s \gg M_{X}^{2}$ as,

$$
\begin{aligned}
s \frac{d^{2} \sigma^{\left(p_{a}+p_{b} \rightarrow p_{c}+X\right)}}{d M^{2} d t} \sim \frac{1}{s} \sum_{i j k} \beta_{p_{a} p_{c}}^{i}(t) \beta_{p_{a} p_{c}}^{j *}(t) \omega_{i}(t) \omega_{j}^{*}(t) & \left(\frac{s}{M_{X}^{2}}\right)^{\alpha_{i}(t)+\alpha_{j}(t)} \\
& \times \beta_{p_{b}}^{k}(0) g_{i j k}^{(3 \mathbb{P})}(t)\left(M_{X}^{2}\right)^{\alpha_{k}(0)},
\end{aligned}
$$


(a)

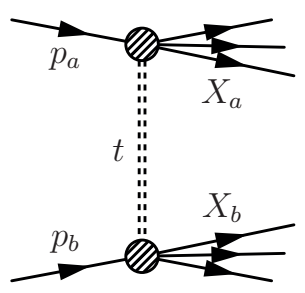

(b)

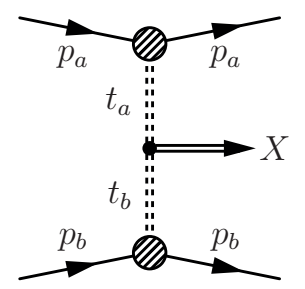

Figure 2.11. Feynman diagrams of (a) double diffraction and (b) of central exclusive production in hadron hadron collisions.

where the $\beta$ terms describe the hadron pomeron vertices, the $\omega$ terms are the signature factors and $g^{(3 \mathbb{P})}$ is the triple pomeron coupling $[\mathbf{4}, \mathbf{4 8}]$. There is zero momentum transfer between the hadron $p_{b}$ and the triple pomeron vertex. One can simplify and factorize the cross section formula for the single diffractive process as particle $p_{a}$ survives the collision and the triple pomeron coupling is approximately independent of $t$.

$$
M_{X}^{2} \frac{d \sigma}{d t d M_{X}^{2}}=\beta_{p_{a}}(t)^{2}|\omega(t)|^{2}\left(\frac{s}{M_{X}^{2}}\right)^{2 \alpha_{\mathrm{P}}(t)-2} \sigma_{p_{b} \mathbb{P}}\left(M_{X}^{2}, t\right),
$$

where $\sigma_{p_{b} \mathbb{P}}\left(M_{X}^{2}, t\right)$ can be seen as the total cross section of hadron $p_{b}$ and the pomeron. The differential cross section with $M_{X}^{2}=\xi s$ where $\xi$ is the energy fraction particle $p_{a}$ lost in the collision [4] is

$$
\frac{d \sigma}{d t d \xi}=\underbrace{\beta_{p_{a}}(t)^{2}|\omega(t)|^{2}\left(\frac{1}{\xi}\right)^{2 \alpha_{\mathbf{P}}(t)-1}}_{\text {Pomeron flux }} \sigma_{p_{b} \mathbb{P}}\left(M_{X}^{2}, t\right) .
$$

Similarly one can formulate differential cross section formulae for double diffraction and central exclusive production. In case of double diffraction, shown in Figure 2.11(a), the formula is driven by a pomeron loop including two triple pomeron vertices,

$$
\begin{aligned}
M_{X_{a}}^{2} M_{X_{b}}^{2} \frac{d \sigma}{d t d M_{X_{a}}^{2} d M_{X_{b}}^{2}}= & \beta_{p_{a}}(0) \beta_{p_{b}}(0) g_{(3 \mathbb{P})}^{2}(0)\left(\frac{s}{M_{X_{a}}^{2} M_{X_{b}}^{2}}\right)^{2 \alpha_{\mathbf{P}}(t)-2} \\
& \times\left(M_{X_{a}}^{2}\right)^{\alpha_{\mathrm{P}}(0)}\left(M_{X_{b}}^{2}\right)^{\alpha_{\mathrm{P}}(0)}
\end{aligned}
$$

Also central exclusive production $(\mathrm{CEP})$ or in other words, double pomeron exchange (DPE), shown in Figure 2.11(b), can be addressed by the Regge formalism $[44,49-52]$. Here one has a pomeron-pomeron fusion producing a system $\mathrm{X}$ and both hadron survive the collision quasi elastically, loosing a fraction of their 
energy $\xi_{a}, \xi_{b}$.

$$
\begin{aligned}
\frac{d \sigma}{d t_{a} d t_{b} d \xi_{a} d \xi_{b}}= & \beta_{p_{a}}\left(t_{a}\right)^{2}\left|\omega\left(t_{a}\right)\right|^{2}\left(\frac{1}{\xi_{a}}\right)^{2 \alpha_{\mathrm{P}}(t)-1} \beta_{p_{b}}\left(t_{b}\right)^{2}\left|\omega\left(t_{b}\right)\right|^{2}\left(\frac{1}{\xi_{b}}\right)^{2 \alpha_{\mathrm{P}}(t)-1} \\
& \times \sigma_{\mathbb{P P}}\left(M_{X}^{2}, t_{a}, t_{b}\right) .
\end{aligned}
$$

The cross section of the pomeron-pomeron fusion $\sigma_{\mathbb{P P}}$ will be discussed later in the context of a QCD approach to CEP as Regge theory is not able to predict it. The central mass system is constrained by $M_{X}^{2}=\xi_{a} \xi_{b} s$. In case the surviving hadrons can be tagged and their momentum losses measured the mass of the central system can be determined with good precision.

Returning shortly to the pomeron, the object with vacuum quantum numbers which has C-parity of +1 . Within the Regge model there is also the possibility of an object with C-parity of -1 and otherwise identical to the pomeron. This object is called odderon and yet no strong evidence for its existence has been found.

Despite some great success the Regge theory has, one should emphasize especially for inelastic processes involving a pomeron with an intercept slightly larger than 1, that one finds a unitarity violation for $s \rightarrow \infty$. More general, all models using the one Pomeron pole will have unitarity violations that leads to a violation of the Froissart(Martin) bound. Most likely one will exceed the black-disc limit already at LHC energies. Of course it might be possible to overcome such difficulties by tuning the model, especially by including a multi pomeron exchange as presented e.g. in [48]. But nonetheless Regge theory is based on a phenomenological approach and yet not fully derivable from first principles which is a necessary step towards the full understanding of diffractive processes.

\subsection{The Pomeron and QCD}

After the advent of QCD first attempts were made to express reggeon or pomeron exchange in terms of a colorless gluon exchange [53-55]. For a colorless exchange at least two gluons are needed for the pomeron as shown in Figure 2.12(a) in case of elastic scattering of hadrons. The odderon can be seen as an exchange of three gluons. This approach leads to some problems. Because of the massless two gluon exchange in a color-singlet configuration one expects a long range Coulomb type potential proportional to some powers of $1 / r$, inconsistent with experiments. For the same reason nature has chosen not to have a strong force theory based on the $U(3)$ group but on the $S U(3)$ group without the color singlet gluon. The breakthrough in describing the Pomeron in terms of perturbative QCD came from the people behind 


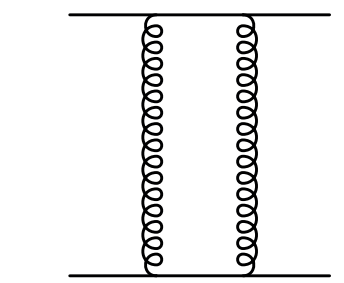

(a)

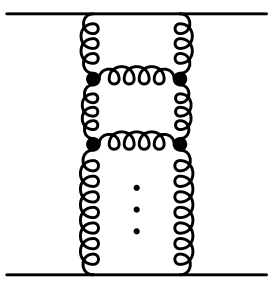

(b)

FiguRE 2.12. Elastic scattering of hadrons via (a) t-channel colorless two-gluon exchange and (b) colorless gluon ladder exchange. Here the dots on the vertices represent the effective Lipatov vertices. The vertical gluons are reggeized.

the BFKL approach (Balitsky-Fadin-Kuraev-Lipatov) $[56-59]^{10}$. All calculations are based on the leading log approximation (LLA) in the low momentum transfer region with $s \gg|t|$. At small momentum transfer $x$ gluons are the dominant partons. The gluons are reggeized with the bare gluon propagator being changed to

$$
D_{\mu \nu}\left(q^{2}\right)=-i \frac{g_{\mu \nu}}{q^{2}} \rightarrow D_{\mu \nu}^{\prime}\left(q^{2}\right)=-i \frac{g_{\mu \nu}}{q^{2}}\left(\frac{s}{s_{0}}\right)^{\varepsilon\left(q^{2}\right)}
$$

where the last factor has the form of a Regge term where the trajectory $\varepsilon$ is now calculable perturbatively. It has been shown that in fact a gluon ladder exchange configured as a color singlet can describe a perturbative Pomeron to some extent. The gluon ladder, as shown in Figure 2.12(b) is a rather complicated summation of the leading terms of infinite ladder diagrams with the vertical gluons being reggeized. Each gluon rung is connected via effective Lipatov vertices to the vertical gluons. This effective vertex includes non-local leading order contributions to the gluon emission at a ladder rung. The final lowest order BFKL equation, an integral equation using the

\footnotetext{
${ }^{10}$ The interested reader might want to look at $[\mathbf{4 5}, \mathbf{6 0}]$ for a detailed introduction to the perturbative pomeron.
} 
Sudakov parametrization for the four momentum vectors ${ }^{11}$ is written as

$$
\begin{aligned}
& \omega f\left(\omega, \boldsymbol{k}_{1}, \boldsymbol{k}_{2}, \boldsymbol{q}\right)=\delta^{2}\left(\boldsymbol{k}_{1}-\boldsymbol{k}_{\mathbf{2}}\right) \\
& +\frac{\bar{\alpha}_{s}}{2 \pi} \int d^{2} \boldsymbol{k}^{\prime}\left[\frac{-q^{2}}{\left(\boldsymbol{k}^{\prime}-\boldsymbol{q}\right)^{2} \boldsymbol{k}_{\mathbf{1}}^{\mathbf{2}}} f\left(\omega, \boldsymbol{k}^{\prime}, \boldsymbol{k}_{\mathbf{2}}, \boldsymbol{q}\right)\right. \\
& +\frac{1}{\left(\boldsymbol{k}^{\prime}-\boldsymbol{k}_{1}\right)^{2}}\left(f\left(\omega, \boldsymbol{k}_{1}, \boldsymbol{k}_{2}, \boldsymbol{q}\right)-\frac{\boldsymbol{k}_{1}^{2} f\left(\omega, \boldsymbol{k}^{\prime}, \boldsymbol{k}_{2}, \boldsymbol{q}\right)}{{\boldsymbol{\boldsymbol { k } ^ { \prime }}}^{\mathbf{2}}+\left(\boldsymbol{k}_{1}-\boldsymbol{k}^{\prime}\right)^{2}}\right) \\
& \left.+\frac{1}{\left(\boldsymbol{k}^{\prime}-\boldsymbol{k}_{\mathbf{1}}\right)^{2}}\left(\frac{\left(\boldsymbol{k}_{\mathbf{1}}-\boldsymbol{q}\right)^{2} \boldsymbol{k}^{\prime 2} f\left(\omega, \boldsymbol{k}^{\prime}, \boldsymbol{k}_{\mathbf{2}}, \boldsymbol{q}\right)}{\left(\boldsymbol{k}^{\prime}-\boldsymbol{q}\right)^{2} \boldsymbol{k}_{\mathbf{1}}^{2}}-\frac{\left(\boldsymbol{k}_{\mathbf{1}}-\boldsymbol{q}\right)^{2} f\left(\omega, \boldsymbol{k}_{\mathbf{1}}, \boldsymbol{k}_{\mathbf{2}}, \boldsymbol{q}\right)}{\left(\boldsymbol{k}^{\prime}-\boldsymbol{q}\right)^{2}+\left(\boldsymbol{k}_{\mathbf{1}}-\boldsymbol{k}^{\prime}\right)^{2}}\right)\right]
\end{aligned}
$$

where $f$ is a Green function, $\boldsymbol{k}_{\mathbf{1}}, \boldsymbol{k}_{\mathbf{2}}$ and $\boldsymbol{k}^{\prime}$ are transverse four momenta carried by the reggeized vertical gluons between the rungs and $q$ the transferred four momentum between the hadrons [45]. The BFKL equation is ultraviolet finite for $\boldsymbol{k}_{\mathbf{1}}, \boldsymbol{k}^{\prime} \rightarrow \infty$ and also shows non divergent behavior in the infrared region for $\boldsymbol{k}_{\mathbf{1}}=\boldsymbol{k}^{\prime}$. Solving the BFKL equation for $t=0$ and applying some simplifications one finds

$$
f\left(\omega, \boldsymbol{k}_{\mathbf{1}}, \boldsymbol{k}_{\mathbf{2}}, 0\right) \approx \frac{1}{2 \pi a k_{1} k_{2}}\left(\frac{k_{1} k_{2}}{\max \left(k_{1}^{2}, k_{2}^{2}\right)}\right)^{\sqrt{\omega-\omega_{0}} / a} \frac{1}{\sqrt{\omega-\omega_{0}}}
$$

where $\omega_{0}=4 \bar{\alpha}_{s} \ln 2$ and $a \approx 14 \bar{\alpha}_{s} \times 1.202$. The last term shows a branch point at $\omega=\omega_{0}$. We can obtain the elastic amplitude for a color singlet exchange in the $s$-dependence at $t=0$ that reads

$$
\frac{A(s, 0)}{s}=4 i \alpha_{s}^{2} \delta_{\lambda^{\prime}{ }_{1} \lambda_{1}} \delta_{\lambda^{\prime}{ }_{2} \lambda_{2}} G_{0} \int \frac{d^{2} \boldsymbol{k}_{\mathbf{1}}}{\boldsymbol{k}_{\mathbf{1}}^{2}} \frac{d^{2} \boldsymbol{k}_{\mathbf{2}}}{\boldsymbol{k}_{\mathbf{2}}^{2}} F\left(s, \boldsymbol{k}_{\mathbf{1}} \boldsymbol{k}_{\mathbf{2}}, 0\right),
$$

where

$$
F\left(s, \boldsymbol{k}_{\mathbf{1}} \boldsymbol{k}_{\mathbf{2}}, 0\right) \approx \frac{1}{\sqrt{\boldsymbol{k}_{1}^{2} \boldsymbol{k}_{\mathbf{2}}^{2}}}\left(\frac{s}{\boldsymbol{k}^{2}}\right)^{\omega_{0}} \frac{1}{\sqrt{\pi \ln \left(\frac{s}{\boldsymbol{k}^{2}}\right)}} \frac{1}{2 \pi a} e^{-\frac{\ln ^{2}\left(\boldsymbol{k}_{1}^{2} / \boldsymbol{k}_{2}^{2}\right)}{4 a^{2} \ln \left(s / \boldsymbol{k}^{2}\right)}}
$$

that one gets by applying the inverse Mellin transform on $f\left(\omega, \boldsymbol{k}_{1}, \boldsymbol{k}_{\mathbf{2}}, 0\right)$. From the amplitude one easily can see the leading log characteristics

$$
\frac{s^{\omega_{0}+1}}{\sqrt{\ln s}} \text {. }
$$

An approximate perturbative pomeron intercept can be extracted

$$
\alpha_{\mathbb{P}}(0)=\omega_{0}+1 \approx 1.5 .
$$

Comparing this pomeron intercept with the one obtained by pure Regge parametrization one can see a stringent difference between the "hard" and the "soft" pomeron. Are they two totally different pomerons or can one find an evolution link between

\footnotetext{
${ }^{11}$ A useful parametrization Sudakov introduced to write the four momentum vector $k^{\mu}$ in terms of the 4-momenta of the scattered hadrons $\left(p_{1}^{\mu}, p_{2}^{\mu}\right)$ and the transverse four momentum to the hadrons $\left(\boldsymbol{k}_{\perp}^{\mu}\right), \boldsymbol{k}^{\mu}=\rho p_{1}^{\mu}+\lambda p_{2}^{\mu}+\boldsymbol{k}_{\perp}^{\mu}$. Here, $\rho$ and $\lambda$ are called Sudakov parameters.
} 


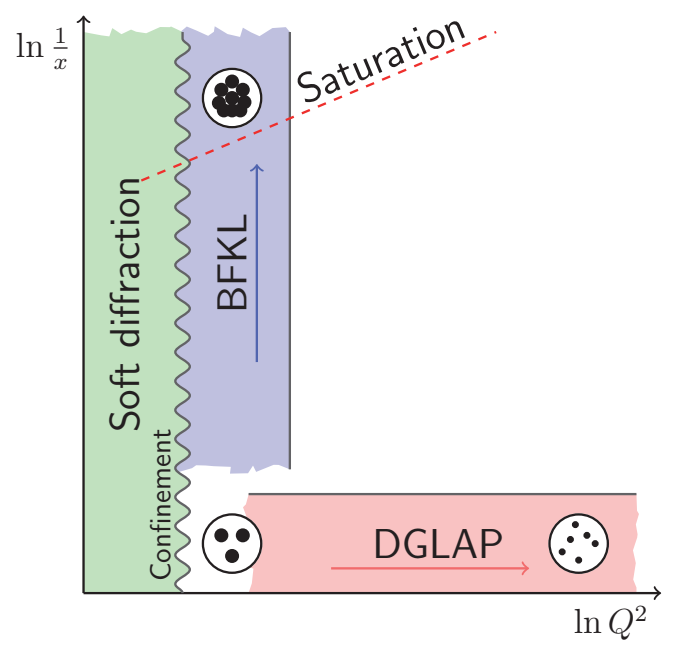

Figure 2.13. Schematic plot of regions in the $\ln Q^{2}-\ln \frac{1}{x}$ plane in which various models work.

them? The LLA will not be able to answer all our questions. Higher order calculations have not been taken into account in this calculation, the strong coupling constant $\alpha_{s}$ has been taken as a non-running constant and most important, the non-perturbative soft pomeron is not understood from first principle physics. The only link between "hard" and the "soft" pomeron so far is provided by experiments.

The BFKL equation can be compared to the DGLAP evolution equations (see Equation (36)). In contrast to DGLAP which is an evolution equation that sums over $\left(\alpha_{s} \ln \left(Q^{2}\right)\right)^{n}$ terms, the BFKL equation sums over $\left(\alpha_{s} \ln (1 / x)\right)^{n}$ terms. In Figure 2.13 the regions in the $\ln Q^{2}-\ln \frac{1}{x}$ plane are schematically marked in which BFLK and DGLAP models are valid. DGLAP works well above $Q^{2}$ of $2 \mathrm{GeV}$ for $x$ down to $10^{-4}$. For increasing $Q^{2}$ the resolution of the partons increases. Towards small $x$ that is described well by the BFKL approach the gluon density grows and we see absorptive effects of the partonic content which leads to complete saturation [61]. For small $x$ multi-pomeron corrections are becoming important for the BFKL evolution. Up to now the transition between those models is still unknown, especially the transition between physics that happens within the confinement radius including the "soft" pomeron and the outer region mainly described by perturbative QCD and its "hard" pomeron. 

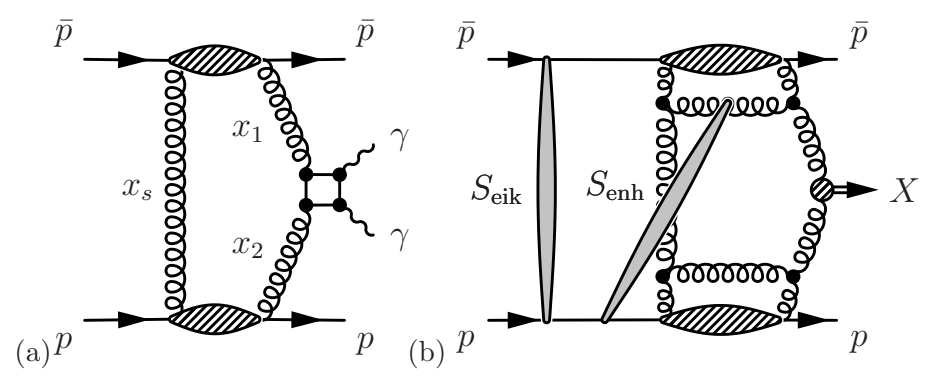

FiguRE 2.14. (a) Leading order diagram for central exclusive productions of $\gamma \gamma$ at hadron colliders. (b) Leading order diagrams for central exclusive productions at hadron colliders. Note the screening gluon to cancel the color flow in the QCD process.

\subsection{Central Exclusive Diffraction and the KMR Model}

Central Exclusive Production (CEP) as already mentioned earlier, is a process that produces some system $X$ in hadron-hadron collisions without the colliding hadrons breaking up, $p_{1} p_{2} \rightarrow p_{1}+X+p_{2}$. The plus signs stand for rapidity gaps between the central system and the outgoing hadrons. It was already briefly discussed how to describe CEP processes in the Regge framework as shown in Equation (77). However, now we focus on a more QCD driven method via t-channel gluon exchange. The method described here refers to the Durham KMR (Khoze-Martin-Ryskin) model [8, $\mathbf{9}, \mathbf{1 7}, \mathbf{6 2 - 6 4}$ ] which is presently the only model describing the key-process of this work, the CEP of a diphoton system at hadron colliders. The example calculation sketched here shows the diphoton production $[\mathbf{1}, \mathbf{2}]$. Very similar are the calculations for a CEP of a dijet system or a SM Higgs. The leading order perturbative calculation for the CEP of $\gamma \gamma$ is shown in Figure 2.14(a). Note the two-gluon t-channel exchange as the lowest order mechanism for a colorless exchange process. The photons are produced via gluon fusion into a light quark loop. The other gluon cancels the color flow and is called screening gluon. Usually the screening gluon carries less momentum than the fusing gluons, $x_{s} \ll x_{1,2} \ll 1[8]$. A more realistic leading order diagram for a CEP of a system $X$ is shown in Figure 2.14(b) that also shows schematically the eikonal and enhanced rescattering which is discussed later.

The amplitude for the CEP process (Figure 2.14(b)) can be written in the form

$$
T=\pi^{2} \int \frac{d^{2} \mathbf{Q}_{\perp} \mathcal{M} \exp \{b(t 1+t 2)\}}{\mathbf{Q}_{\perp}^{2}\left(\mathbf{Q}_{\perp}-\mathbf{p}_{1_{\perp}}\right)^{2}\left(\mathbf{Q}_{\perp}+\mathbf{p}_{2_{\perp}}\right)^{2}} f_{g}\left(x_{1}, x_{1}^{\prime}, Q_{1}^{2}, \mu^{2}\right) f_{g}\left(x_{2}, x_{2}^{\prime}, Q_{2}^{2}, \mu^{2}\right),
$$

where $Q_{\perp}$ is the transverse gluon momentum in the inner fusion process, $p_{1 \perp}, p_{2 \perp}$ the transverse momenta of the outgoing hadrons, $\mathcal{M}$ the matrix element of the inner fusion 


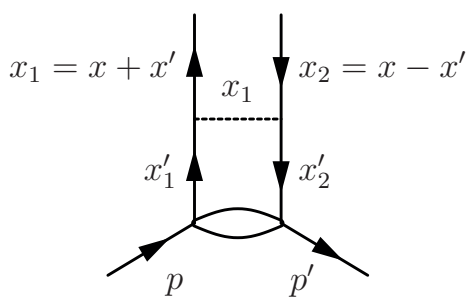

FiguRE 2.15. Diagram illustrating the off-diagonal parton distribution $f\left(x, x^{\prime}\right)[66]$

process and the $f_{g}\left(x, x^{\prime}, Q_{\perp}^{2}, \mu^{2}, t\right)$ terms inside the intgral are the generalized skewed unintegrated gluon densities of the hadrons. The term $\exp \{b(t 1+t 2)\}$ describes the proton vertex with $b$ being the slope of the t-distribution of the colliding hadrons. The authors of the present calculation use a slope $b=4 \mathrm{GeV}^{2}$.

The differential cross section is calculated by integrating over the transverse momenta of the outgoing hadrons.

$$
\frac{d \sigma}{d y_{X}}=\frac{1}{16^{2} \pi^{5}} \int d^{2} \mathbf{p}_{1 \perp} d^{2} \mathbf{p}_{2 \perp}\left|T\left(\mathbf{p}_{1 \perp}, \mathbf{p}_{2 \perp}\right)\right|^{2} S_{\mathrm{e} i k}^{2}\left(\mathbf{p}_{1 \perp}, \mathbf{p}_{2 \perp}\right)
$$

Here the rapidity gap survival factor $S_{\text {eik }}^{2}$ (eikonal screening) is introduced that accounts all soft rescattering occurring in the CEP process that is schematically shown in Figure 2.14(b) as the grey blob connecting the incoming hadrons.

2.4.1. Generalized Unintegrated Parton distributions. The generalized unintegrated parton densities can be calculated from conventional parton distributions $g\left(x, \mu^{2}\right)$ and $q\left(x, \mu^{2}\right)[65]$. Generalized or skewed means introducing a new variable $x^{\prime}$ to describe off-diagonal parton distributions important for diffractive processes. In Figure 2.15 one can see the meaning of $x^{\prime}$ acting on the momentum fraction $x$ carried by the parton. The meaning of unintegrated refers to the parton density distributions that are unintegrated over the parton transverse momentum $Q_{\perp}$ and enable a more exact kinematical calculation of the central hard scale subprocess. It is possible to obtain such parton distributions from conventional ones. The procedure described in detail in [67] starts from DGLAP evolution over the scale $\mu^{2}$. Real emissions of partons modifies the transverse momentum $k_{t}$ of a parton quite to the contrary of virtual parton emissions. Re-summing of the latter results in a survival factor called Sudakov suppression that works up to the scale $\mu^{2}$ of the hard scale subprocess. The result is

$$
f_{a}\left(x, x^{\prime} ; Q_{\perp}^{2}, \mu^{2}\right)=\sqrt{T_{1}^{a} T_{2}^{a}}\left[\frac{\alpha_{S}\left(Q_{\perp}^{2}\right)}{2 \pi} \sum_{a^{\prime}} \int_{x}^{1-\bar{\Delta}} \frac{d z}{z} P_{a a^{\prime}}\left(z, \frac{x^{\prime} z}{x}\right) F_{a^{\prime}}\left(\frac{x}{z}, x^{\prime}, Q_{\perp}^{2}\right)\right]
$$


where

$$
T_{i}^{a}=\exp \left(-\int_{Q_{\perp}^{2}}^{\mu^{2}} \frac{d Q_{\perp}^{\prime 2}}{Q_{\perp}^{\prime 2}} \frac{\alpha_{S}\left(Q_{\perp}^{\prime 2}\right)}{2 \pi} V_{i}^{a}\left(\Delta_{i}\right)\right)
$$

is the Sudakov factor. The index $a$ stands for a parton, either a gluon or a quark. $P_{a a^{\prime}}$ are the parton splitting functions that were introduced earlier already. The generalized skewed parton densities enter as $F_{a^{\prime}}\left(x / z, x^{\prime}, Q_{\perp}^{2}\right)$ inside the integral over $z$ up to a cut off $\bar{\Delta}$. The cut offs (in addition $\Delta_{1 / 2}$ ) are introduced to deal with singularities due to soft gluon emissions.

2.4.2. Rapidity Gap Survival Factors. The rapidity gap survival factor that we mentioned already is an essential part of the effective luminosity estimation for a CEP process. Without going to deep into the matter (a detailed discussion of the gap survival factor calculated in a generalized two-channel eikonal model can be found in $[68,69]$ and references therein) the eikonal gap survival factor $S_{\text {eik }}^{2}$ is now briefly introduced. Integrated over the impact parameter $b$ one has

$$
S_{\text {eik }}^{2}=\int \frac{\sum_{i}\left|\mathcal{M}_{i}\left(s, b_{t}^{2}\right)\right|^{2} \exp \left(-\Omega_{i}\left(s, b_{t}^{2}\right)\right)}{\sum_{i}\left|\mathcal{M}_{i}\left(s, b_{t}^{2}\right)\right|^{2}} d^{2} b_{t}
$$

where $\mathcal{M}_{i}$ refers to the amplitudes of the hard subprocess producing system $X$. The amplitudes vary for the different diffractive eigenstates. Despite the calculability in pQCD of the matrix element, its shape and couplings to the soft diffractive eigenstates is unknown and has to be estimated partly from experiments. The proton opacity $\Omega$ may be seen as the probability for no inelastic scattering at a certain impact parameter $b_{t}$. The gap survival factor grows with increasing $b_{t}$. At Tevatron $S_{\text {eik }}^{2}$ is of the order of 0.05 for a slope parameter $B=4 \mathrm{GeV}^{-2}$. The survival factor divided by the slope parameter is approximately constant. The eikonal gap survival factor does depend on the type of CEP process, the size of the rapidity gap and the impact parameter $b$.

The enhanced rescattering factor $S_{\text {enh }}$ also sketched in Figure 2.14 includes rescattering effects mainly by intermediate partons that are described by the unintegrated gluon distributions. This factor is especially non-negligible for low $x$ values. It has a rather small dependence on the impact parameter $b$. Detailed information can be found in reference [69] and references therein.

2.4.3. Subprocess. The matrix element $M$ of the subprocess inside Equation (85) can be written in more details:

$$
\mathcal{M} \equiv \frac{2}{M_{X}^{2}} \frac{1}{N_{C}^{2}-1} \sum_{a, b} \delta^{a b} q_{1_{\perp}}^{\mu} q_{2_{\perp}}^{\nu} V_{\mu \nu}^{a b},
$$

where $M_{X}$ is the mass of the produced central system, $V_{\mu \nu}$ the $g g \rightarrow \gamma \gamma$ vertex and $q_{1 \perp}, q_{2 \perp}$ are the transverse momenta of the fusing gluons. The matrix element is 


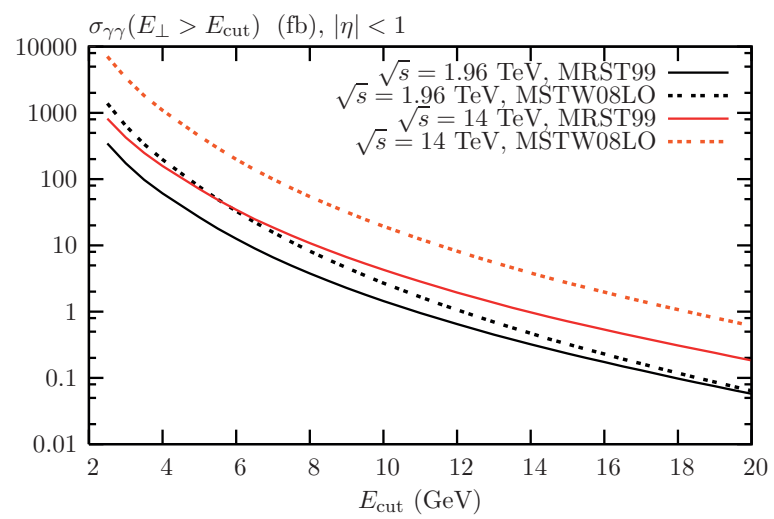

FiguRE 2.16. Cross section estimates for exclusive $\gamma \gamma$ production versus the $E_{\text {cut }}$ of the produced photons using the KMR model. Predictions for Tevatron and design LHC energies using two different PDF's (MRST99, MSTW08LO) are given. Plot is taken from [2].

summed over all color combinations with $a, b$ being the color indices. The integration is cut off for transverse momenta below $0.85 \mathrm{GeV}$ to ensure reliable perturbative results for the hard process calculation entering the cross section estimate. Due to the color singlet exchange in the hadron collision with little longitudinal momentum losses of the outgoing hadrons one can expect a certain spin and quantum state of the produced system. The transverse polarized fusing gluons will not build up a state with an angular momentum in z direction as the colliding hadrons tend to carry no transverse momenta (very small angle scattering). Here $z$ denotes the direction of the colliding hadron beams. The centrally produced system has to be therefore in a $J_{z}^{P C}=0^{++}$ state. However, the fact that the transverse momenta of the outgoing hadrons $p_{1 \perp}, p_{2 \perp}$ are not totally zero (but small) will give us a small contribution from the $J_{z}=2$ state at the $1 \%$ level $[\mathbf{2}]$.

2.4.4. Predictions for $\boldsymbol{C E P}$ Processes. Predictions had been made by the authors of the above sketched model for Tevatron and the LHC with certain configurations. In case of CEP of photon-pairs, that covers the experimental studies of this thesis, the results are shown in Figure 2.16. The cross section calculation is done for Tevatron and design LHC energies for central photons with a pseudorapidity between $-1<\eta<1$ as a function of an minimal energy cut $E_{\text {cut }}$ applied to the photons in the analysis. Easily one can spot the huge dependence upon the choice of the unintegrated generalized PDF's used for the predictions. 


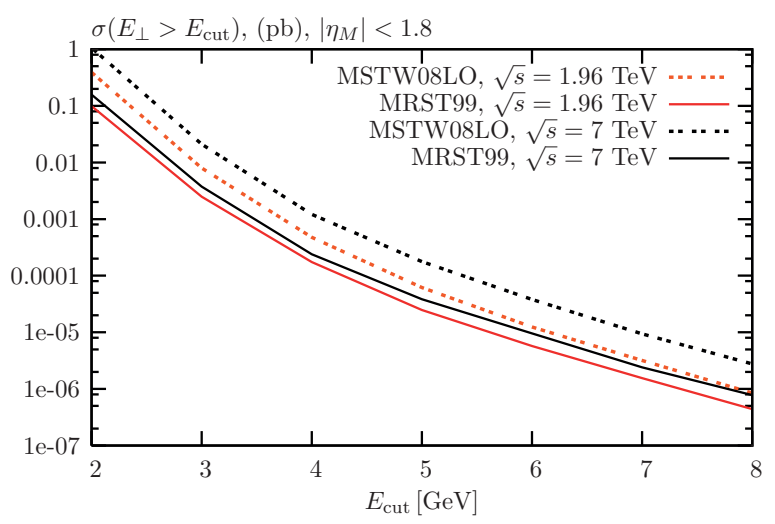

Figure 2.17. Cross section estimates for exclusive $\pi^{0} \pi^{0}$ production using the KMR model. The cross section is plotted as a function of $E_{\text {cut }}$ on the produced mesons. Predictions for Tevatron and design LHC energies using two different PDF's (MRST99, MSTW08LO) are given. Plot is taken from [70].

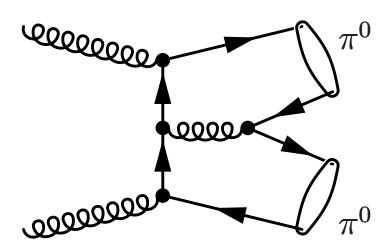

FiguRE 2.18. A leading order diagram for the $g g \rightarrow \pi^{0} \pi^{0}$ process.

As it turns out (to be discussed later in detail), the exclusive production of neutral pion pairs is of essential importance in this study as a possible background to the exclusive photon-pair production. Predictions of the CEP of neutral pion-pairs are shown in Figure 2.17. One can immediately see that the production rate is some magnitudes of order smaller than for the diphoton production. Theoretically at first glance it seems that the CEP of $\pi^{0} \pi^{0}$ would dominate over the $\gamma \gamma$ process. Looking at a typical leading order diagram as shown in Figure 2.18 one can see four strong coupling vertices each proportional to $\alpha_{s}$. In case of the diphoton production one has two strong coupling vertices and two QED coupling vertices proportional to $\alpha$ that is much smaller. However, the overall production cross section for exclusive neutral pion pairs in reality is highly suppressed compared to the diphoton channel (compare Figure 2.17 and Figure 2.16) due to the special configuration and selection rules of the CEP processes. The incoming gluons are in a helicity state that favor creating $J_{z}=0$ states but in case of the production of $\pi^{0} \pi^{0}$ final states only the $J_{z}=2$ 


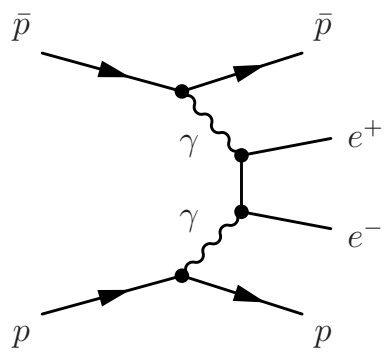

FiguRE 2.19. Leading order diagram for central exclusive production of $\gamma^{*} \gamma^{*} \rightarrow e^{+} e^{-}$at a proton-antiproton collider.

amplitudes contribute that leads to the strong suppression. An additional suppression for the production of mesons with high $k_{\perp}$ comes from the small factor $\left(f_{M} / k_{\perp}\right)^{4}$, where $f_{M}$ is the mesons decay constant or form factor. Details can be found in [70].

Last but not least a view words to the CEP of a SM Higgs boson [71-73]. Replacing the inner light quark loop in the gluon fusion hard process of the diphoton production with a heavy quark triangle one can estimate the cross section at hadron colliders. Even though not feasible at the Tevatron one might well be able to detect such a boson at the LHC taken as granted that forward proton-taggers at $420 \mathrm{~m}$ from the IP and forward veto counters are installed $[\mathbf{7 5}, 76]$. A $120 \mathrm{GeV}$ SM Higgs boson has a predicted cross section of $3 \mathrm{fb}$ with a factor $\stackrel{\times 3}{\div 3}$ uncertainty at $\sqrt{s}=14 \mathrm{TeV}[\mathbf{7 1}]^{12}$. A feasibility study of detecting a central exclusive produced SM Higgs boson decaying to $b \bar{b}$ quarks at the LHC can be found in [78].

Due to its similar production mechanism the CEP of the SM Higgs boson can be tested at Tevatron by searching for the CEP of diphotons which is therefore called a standard candle CEP.

\section{Photo Induced Central Exclusive Production}

CEP can also be photo induced at hadron colliders. In hadron-hadron collisions virtual photons can be emitted from both hadrons that produce a central massive system $X, p+p \rightarrow p+\gamma^{*} \gamma^{*}+p \rightarrow p+X+p$. Here the + -sign denotes rapidity gaps between the outgoing surviving hadrons and the produced system. An example diagram is shown in Figure 2.19 sketching the photon induced production of electronpositron pairs at a proton-antiproton collider. The differential cross section for such a

\footnotetext{
${ }^{12}$ The latest update on the CEP cross section expectations for a light SM Higgs boson can be found in $[77]$
} 
QED mediated process can be calculated by

$$
\frac{d \sigma}{d \Omega}=\int \frac{d \sigma_{\gamma^{*} \gamma^{*} \rightarrow X}(W)}{d \Omega} \frac{d L^{\gamma^{*} \gamma^{*}}}{d W} d W
$$

where $W$ is the center of mass energy of the virtual photons and $L^{\gamma^{*} \gamma^{*}}$ the effective luminosity of the fusing virtual photons [75]. The cross section of the pure QED subprocess $\sigma_{\gamma^{*} \gamma^{*} \rightarrow X}$ is precisely calculable. The virtual photon luminosity can be obtained by using a method known as the equivalent photon approximation (EPA) that is described in detail in [79]. The photon spectrum for proton-antiproton collisions is given by

$$
d n=\frac{\alpha}{\pi} \frac{d E_{\gamma^{*}}}{E_{\gamma^{*}}} \frac{d\left(-q^{2}\right)}{\left|q^{2}\right|}\left[\left(1-\frac{E_{\gamma^{*}}}{E}\right)\left(1-\frac{\left|q_{\min }^{2}\right|}{\left|q^{2}\right|}\right) \frac{4 m_{p}^{2} G_{E}^{2}-q^{2} G_{M}^{2}}{4 m_{p}^{2}-q^{2}}+\frac{E_{\gamma^{*}}^{2}}{2 E^{2}} G_{M}^{2}\right] .
$$

where $\alpha$ is the electromagnetic coupling constant, $E$ is the (anti)proton energy, $E_{\gamma^{*}}$ the energy of the virtual photon, $-q^{2}$ the photon virtuality and $m_{p}$ the mass of the (anti)proton. $G_{E}$ and $G_{M}$ are the electric and magnetic form factors of the (anti)proton in the dipole approximation.

For the cross section estimation of photon induced processes also soft QCD rescattering effects have to be taken into account. Even though the QED part of the process is well known, the colliding particles are highly complex objects with a not very precisely known inner structure. However, due to the larger impact parameter $b$ at which those processes occur one expects a much larger gap survival probability compared to pure QCD mediated CEP processes. At Tevatron energies for pure exclusive QED production of $e^{+} e^{-}$with $p_{T} \gg m_{e}$ the gap survival probability is in fact maximal as rescattering effects can be neglected $[\mathbf{8 0}, \mathbf{8 1}]$; it would be different for massive central states as a low mass Higgs boson at $1.96 \mathrm{TeV}$. Here the survival probability would be around 0.75 . In case of non pure CEP production with one or the two colliding hadrons being excited, the survival factor has to be taken into account [3].

A third possibility of CEP processes at hadron colliders are photon-pomeron fusion processes (called photoproduction) such as for example $p+p \rightarrow p+\gamma^{*} \mathbb{P}+p \rightarrow p+J / \Psi+p$ that has been observed successfully at Tevatron by the CDF collaboration [82]. This was also observed very recently at the LHC by the LHCb collaboration [83]. Details about photoproduction can be found in reference [84] and references therein. 


\section{CHAPTER 3 \\ Experimental Setup}

\section{Fermilab}

Fermilab, a US Department of Energy national laboratory near Chicago, is a key site for experimental high energy physics. The Tevatron collider at Fermilab provided the highest energy hadron-hadron collisions for the physics community until September 2008 when CERN's Large Hadron Collider started. At Tevatron two multipurpose detectors, $\mathrm{D} \emptyset$ and CDF had been installed at two intersection points.

\subsection{Accelerator Complex}

The accelerator apparatus consists of a production site, an accumulator for antiprotons, the Tevatron and several beamlines for various purposes. A schematic drawing of the whole complex is presented in Figure 3.1. Before protons and antiprotons can spin in the Tevatron, they have to undergo a production chain as explained below.

1.1.1. Proton and Antiproton Production. Protons are produced from hydrogen gas. Negatively charged hydrogen ions are pre-accelerated to $750 \mathrm{KeV}$ by a Cockcroft Walton accelerator. A subsequent linear accelerator (Linac) accelerates the ions up to an energy of $400 \mathrm{MeV}$ before they are injected into the booster, a synchrotron type of accelerator with a radius of $75 \mathrm{~m}$. This machine strips the electrons off the hydrogen ions and accelerates the remnant protons up to $8 \mathrm{GeV}$. From the booster the protons are injected via the MI-8 line into the main injector (MI), a circular synchrotron with a circumference greater than $3.3 \mathrm{~km}$. The MI can be run under different operational modes: (a) it provides beam for external neutrino experiments (among others) and (b) it serves the Tevatron by injecting protons that are accelerated up to $150 \mathrm{GeV}$ (injection mode), and (c) by sending $120 \mathrm{GeV}$ protons to the antiproton source (stacking mode). The antiproton production can be divided into several steps. First, protons from the MI hit a nickel alloy target, then from the resulting showers of secondary particles $8 \mathrm{GeV}$ antiprotons are collected and send to the debuncher, a triangular shaped synchroton (see Figure 3.1). At the same position one can find the accumulator, which stores the $8 \mathrm{GeV}$ antiprotons from the debuncher and additionally cools them down. Next the antiprotons are sent shotwise to the recycler. The recycler, 


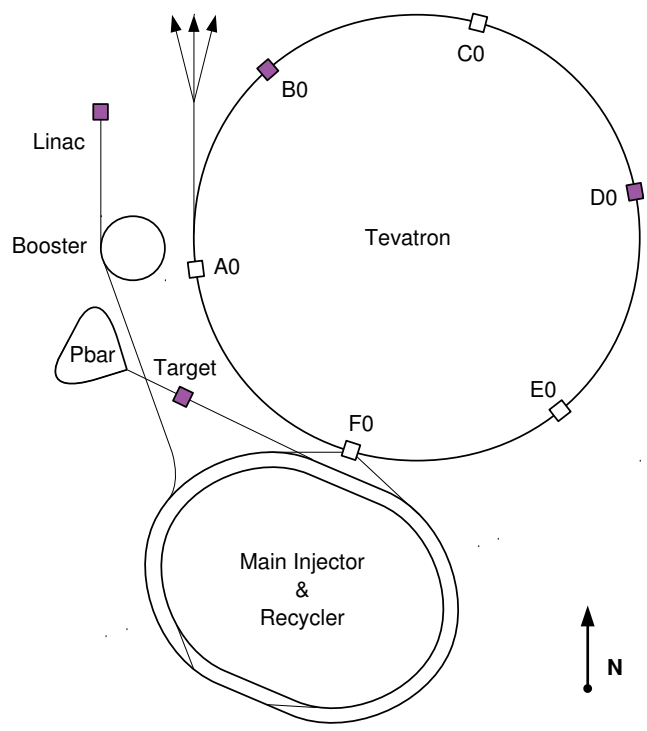

FiguRE 3.1. Overview of the Fermilab accelerator complex including the machinery for proton and antiproton production as well as the intermediate storage ring and the Tevatron. The CDF experiment is located at B0.

an antiproton storage ring, is built in the same tunnel as the MI and accumulates the $8 \mathrm{GeV}$ antiprotons until a certain stash size is reached. It uses stochastic and active electron cooling. The name recycler comes from the ability of recycling the remaining antiprotons after a Tevatron-store has finished. In shot setup mode the antiprotons are extracted to the MI, pre-accelerated and injected into the Tevatron. Even though the antiproton production has seen large improvements over the years, it remains the bottle-neck in producing high luminosities at the Tevatron.

1.1.2. Tevatron. The Tevatron itself is a synchrotron type of accelerator and storage ring with a circumference of approximately $6.28 \mathrm{~km}$ length. Using radio frequency oscillators protons and antiprotons are accelerated up to $980 \mathrm{GeV}$ from the injection energy of $150 \mathrm{GeV}$ in opposite directions. Superconducting magnets are used to keep the (anti)protons on track. Injected from the MI in three bunch trains of 12 bunches one has in total 36 bunches of (anti)protons spinning in the Tevatron. The time between bunch crossings is $396 \mathrm{~ns}$. A bunch is approximately filled by the order of $10^{13}$ protons and of $10^{12}$ antiprotons. The instantaneous luminosity can be obtained 


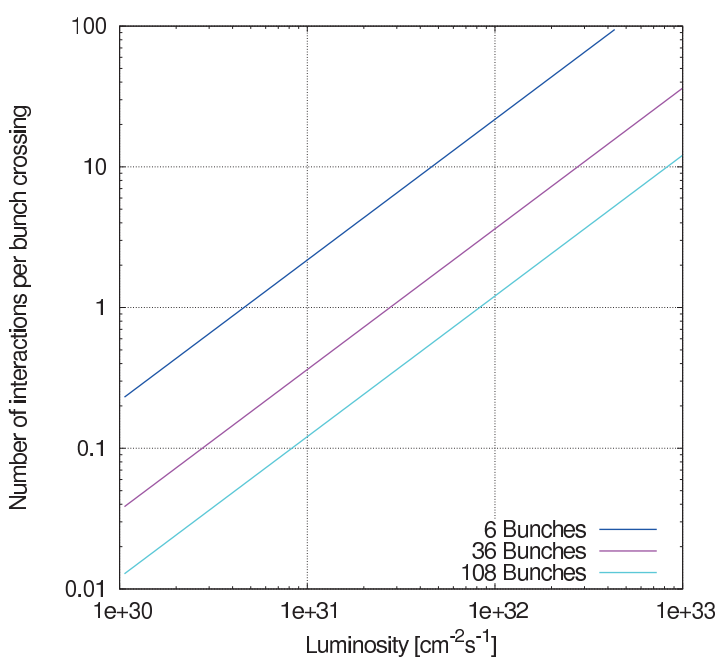

FiguRE 3.2. Average number of interactions per single bunch crossing for different numbers of filled bunches in the Tevatron.

by,

$$
\mathcal{L}_{\text {inst }}=\frac{f N_{b} N_{p} N_{\bar{p}}}{4 \pi \sigma_{x} \sigma_{y}}
$$

where $f$ is the beam oscillation frequency, $N_{b}$ the number of bunches, $N_{p}$ and $N_{\bar{p}}$ the number of protons and antiprotons per bunch respectively and $\sigma_{x}$ and $\sigma_{y}$ the Gaussian profile of the transverse beam size in $x$ and $y$. The maximum instantaneous luminosity reached during Run II was $414 \times 10^{30} \mathrm{~cm}^{-2} \mathrm{~s}^{-1}$. The Tevatron has two interaction points (IP) where bunches are brought to collision serving the two multipurpose particle detectors, namely D $\emptyset$ at IP D0 and CDF at IP B0 as shown in Figure 3.1. The average number of $p \bar{p}$ interactions per bunch crossing is shown for different number of filled bunches in Figure 3.2. For the calculation of the average number of proton-antiproton interactions the Tevatron beam oscillation frequency $f=46500 \mathrm{~Hz}$ and the total inelastic cross section of CDF $\sigma_{\text {inel }}=61 \mathrm{mb}$ was used. At instantaneous luminosities around $300 \times 10^{30} \mathrm{~cm}^{-2} \mathrm{~s}^{-1}$ for example, pileup ${ }^{1}$ occurs with an average number of 10 interactions per bunch crossing in the case of 36 filled bunches in the Tevatron.

\footnotetext{
${ }^{1}$ It is called pileup if more than one $p \bar{p}$ interaction occurs in one single bunch crossing.
} 


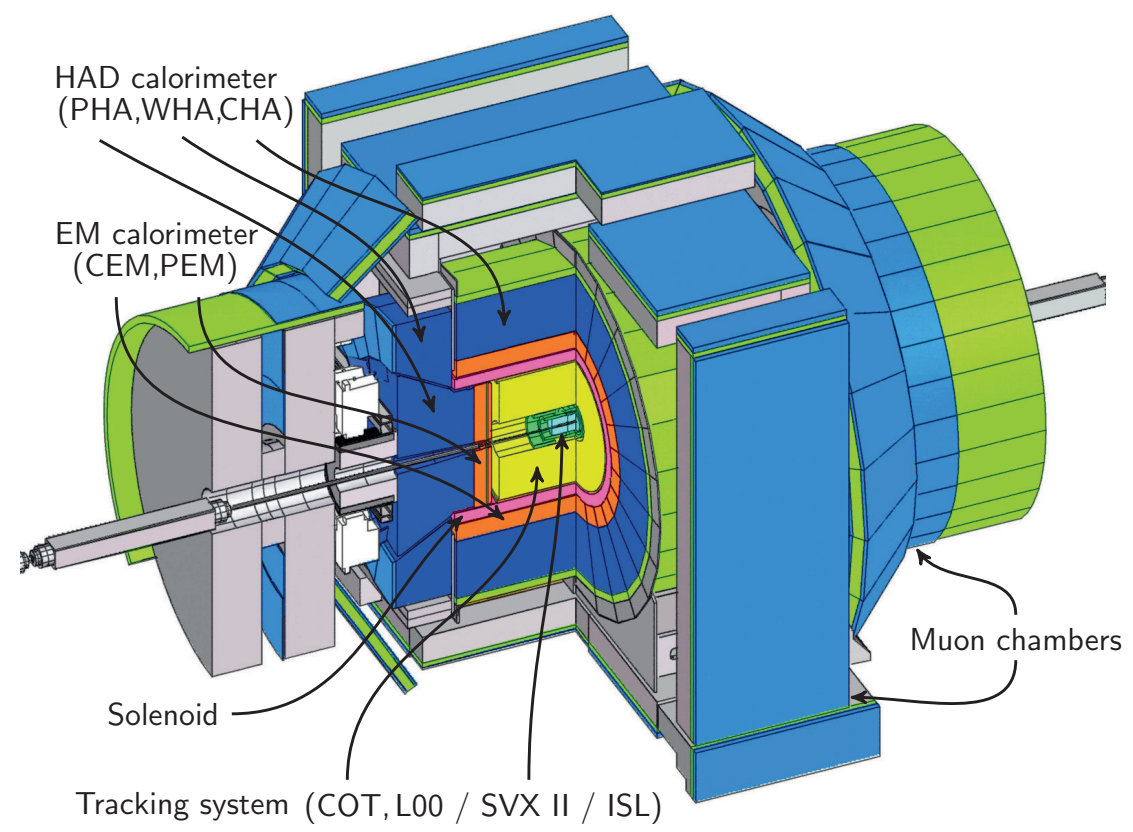

Figure 3.3. Isometric cutaway view of the CDF Run II detector at Tevatron/Fermilab. (Taken and modified from the TDR [85]).

\section{Collider Detector at Fermilab}

The Run II Collider Detector at Fermilab ${ }^{2}$ (CDF) $[\mathbf{8 6 - 8 9}]$ is the successor of the Run I detector that collected data leading to the top quark discovery in the year 1995 . CDF Run II started operation in the year 2001 and has collected an impressive amount of collision data ${ }^{3}$ until Tevatron was shutdown in the end of September 2011.

The CDF II detector consisting of various subdetectors (see Figure 3.3 for an isometric view or Figure 3.4 for an elevation view of the CDF II) is a general multipurpose detector with azimuthal and forward backward symmetry. The detector is build using the standard principle of particle detection that is shown schematically in Figure 3.5. Starting from the inside, one finds a tracking system surrounding the beampipe, consisting of a silicon microstrip detector and a cylindrical drift chamber all within a liquid helium cooled superconducting solenoid with a magnetic flux density

\footnotetext{
${ }^{2} \mathrm{~A}$ detailed description of the whole CDF II detector including physics goals, can be found in the technical design report [85].

${ }^{3}$ The total recorded data using a complete detector configuration corresponds to $10 \mathrm{fb}^{-1}$ of integrated luminosity.
} 


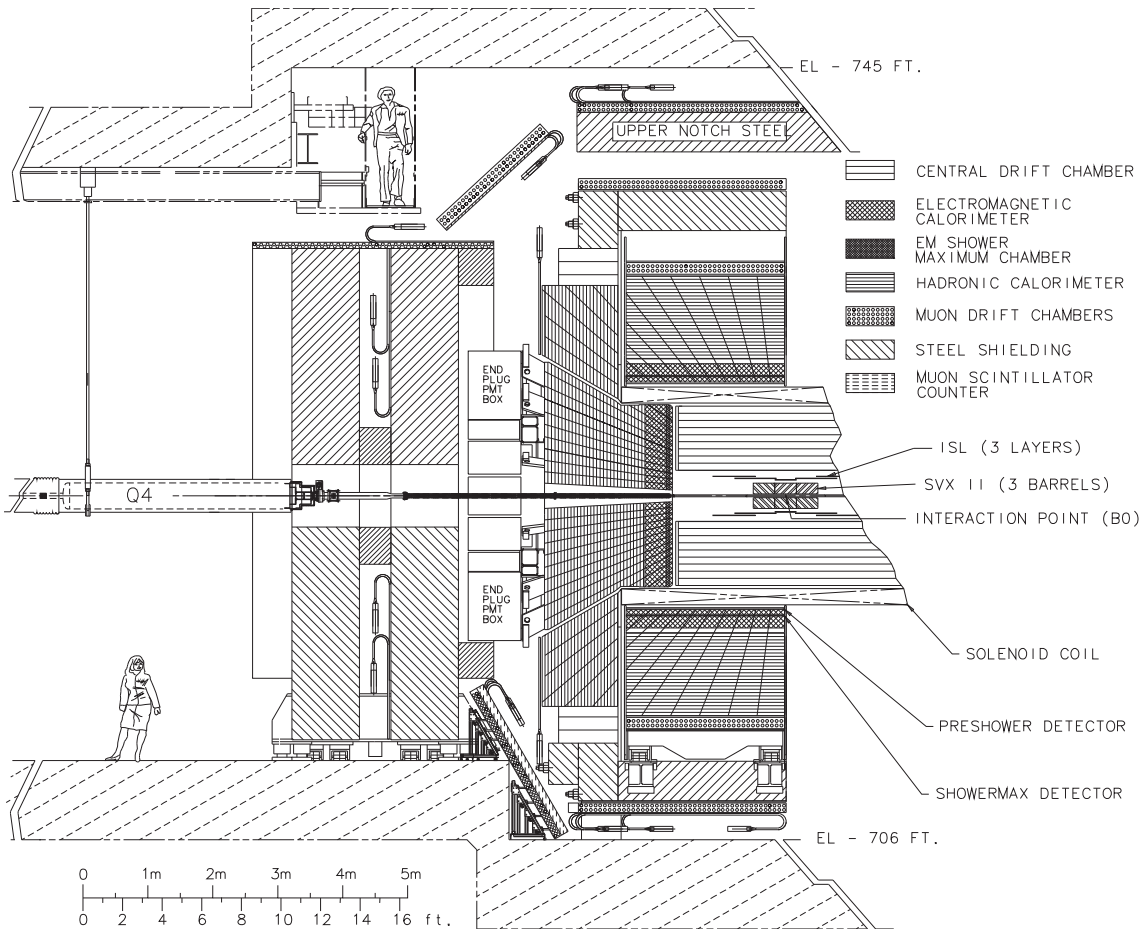

Figure 3.4. Elevation view of the CDF Run II detector at Tevatron/Fermilab. (Taken from the TDR [85]).

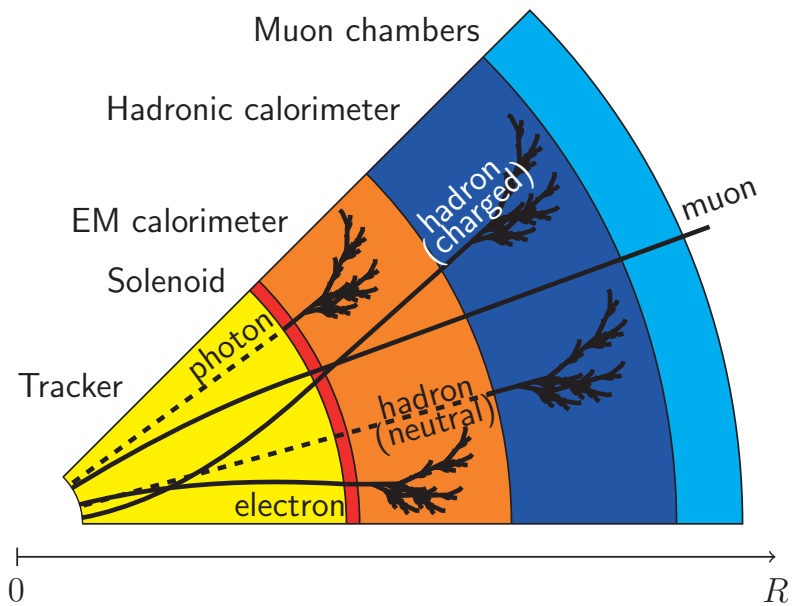

FIGURE 3.5. Principles of particle detection. 


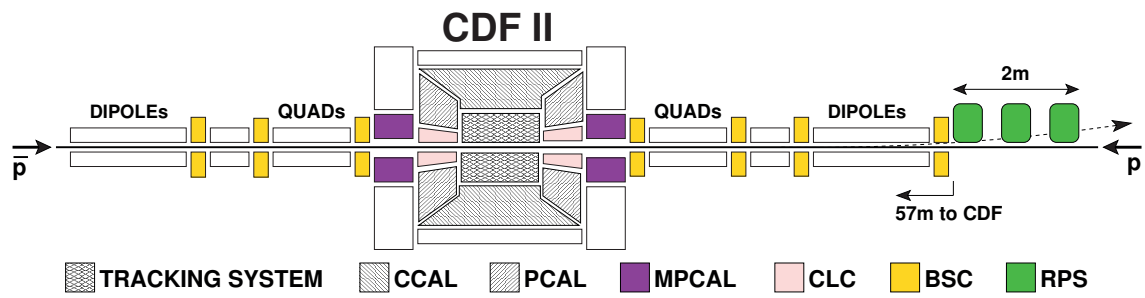

Figure 3.6. Schematic unscaled view of the forward detectors of CDF Run II. On the east side only three beam shower counter stations are installed and no Roman Pots due to space limitations. (Drawing taken from $[90])$.

of 1.4 Tesla. The tracking device measures the momentum and direction of charged particles, whereas neutral particles are not detected. Outside the solenoid a electromagnetic and hadronic calorimetry is installed that is extended into the forward, plug region $(1.1<|\eta|<3.6)$. Particles such as electrons will deposit their energy mostly in the electromagnetic (EM) calorimeter and hadronic particles such as charged pions or protons mostly in the hadronic calorimeter. Additionally a muon chamber system is build outside the calorimetry. Muons are not absorbed by the heavy material of the calorimeter and are practically the only type of particles that leave some track in the outermost detection layer. Using all the information from the tracker, the EM and hadronic calorimeter, the muon chamber, etc., stable or long lived particles can mostly be identified and categorized at early stages. An exception are neutrinos that pass the entire detector with very low interaction probability with the material. Those are accounted by reconstructing the missing transverse energy MET.

The CDF II detector has additionally a forward extension consisting of Cherenkov luminosity counters (CLC), a forward calorimeter (Miniplug or MPCAL), several beam shower counter stations (BSCs) and Roman $\operatorname{pots}^{4}$ (RPS) (see Figure 3.6). Those detectors are crucial for diffractive physics as they cover a large region of rapidity. The CLC is mainly used to measure the delivered instantaneous luminosity. The Miniplug measures the forward energy flow of neutral and charged particles and the BSCs are mostly used as veto counters. The Roman pots are tracking detectors that operate inside the beampipe at large distance to the interaction point to detect small-angle scattered antiprotons.

\footnotetext{
${ }^{4}$ Due to the Tevatron machine layout the Roman pots had only be installed on the antiproton side.
} 


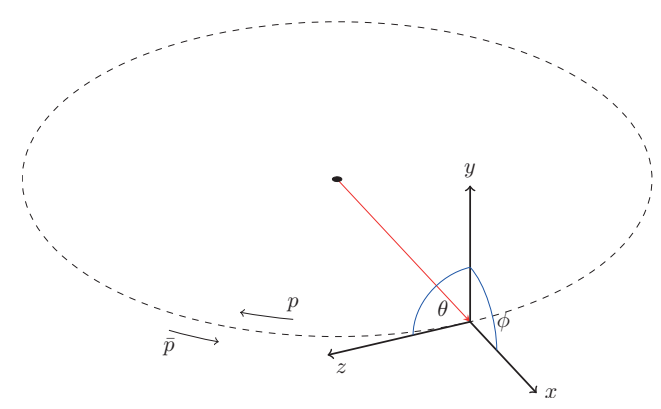

Figure 3.7. The CDF coordinate system.

\subsection{Coordinate System}

At CDF the coordinate system used is a right handed Cartesian with the $z$-axis pointing into the proton beam direction (east) with $z=0$ at the IP, the $x$-axis point outside the Tevatron ring to the north and the $y$-axis vertically upwards (see Figure 3.7). More suitable for barrel shaped detectors is a cylindrical coordinate system. In addition to the $z$-coordinate the radial distance from the beamline $r=\sqrt{x^{2}+y^{2}}$, the azimuthal angle $\phi$ starting from $\phi=0$ along the $x$-axis and the polar angle $\theta$ with $\theta=0$ along the positive $z$-axis are used. Instead of the polar angle $\theta$ often the pseudorapidity $\eta$ is used. The pseudorapidity $\eta$ is defined as,

$$
\eta=-\ln \tan \frac{\theta}{2}
$$

where $\theta$ is the polar angle. Equation (94) is an approximation of the rapidity ${ }^{5} y$,

$$
y=\frac{1}{2} \ln \frac{E+p_{z}}{E-p_{z}}
$$

in cases with the momentum of particles being much greater than their masses, $p \gg m$. Here $E$ is the energy of particles and $p_{z}$ their longitudinal momentum in $z$-direction. The pseudorapidity $\eta$ is zero perpendicular to the beamline and goes towards $\pm \infty$ parallel to the beamline. In terms of pseudorapidity $\eta$ the central detector covers $|\eta|<1.1$ and the plug region ranges from $1.1<|\eta|<3.6$. Often used are the transverse energy $E_{T}=E \sin \theta$ and transverse momentum $p_{T}=p \sin \theta$, the azimuthal momentum-vector component. For measuring distances in the $\eta$ and $\phi$ space the quantity $\Delta R$ is used, which is defined as

$$
\Delta R=\sqrt{(\Delta \eta)^{2}+(\Delta \phi)^{2}} .
$$

\footnotetext{
${ }^{5}$ Rapidity differences are invariant under boosts along the $z$-axis. As a consequence the number of particles per unit of rapidity $d n / d \eta$ is invariant under such boosts.
} 


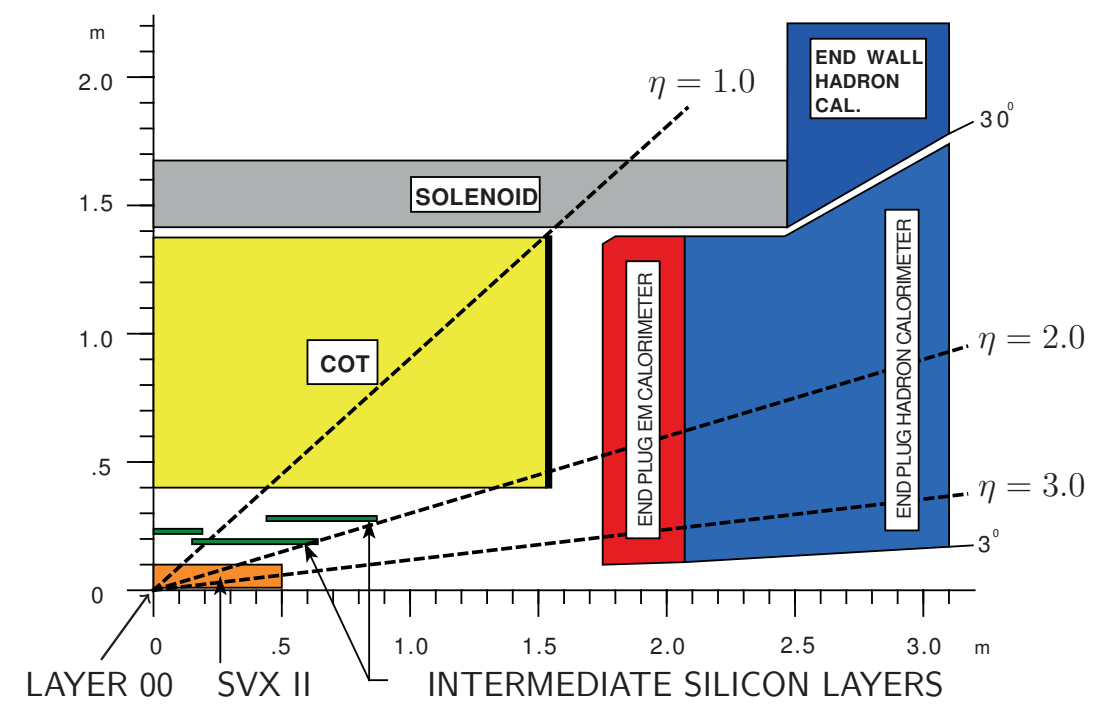

FiguRE 3.8. Sketch of the CDF Run II tracking system (cut in the $z-y$ plane). Starting from the innermost component, the tracker consists of the Layer 00 silicon microstrip detector (L00), the silicon vertex detector (SVX II) and the cylindrical drift chamber (COT). (Taken and modified from the TDR $[85]$ ).

In the following all subdetector systems will be introduced in more detail, starting with the tracking system.

\subsection{Tracking System}

The CDF II tracking system consists of a silicon tracker in the most inner part and a cylindrical drift chamber (called Central Outer Tracker, COT) surrounded by a solenoid of 1.4 Tesla to ensure good momentum measurements of charged particles from the IP. In Figure 3.8, a sketch of the CDF II tracking system is shown.

2.2.1. Silicon Tracker. The silicon tracker with its main purpose of high precision tracking and detection of secondary vertices, is split into three different cylindrical subdetectors, the Layer 00, the SVX II and the ISL, as shown in Figure 3.9(a). Common to all are the solid state type p-n silicon modules that record the path of ionizing particles that pass through. 


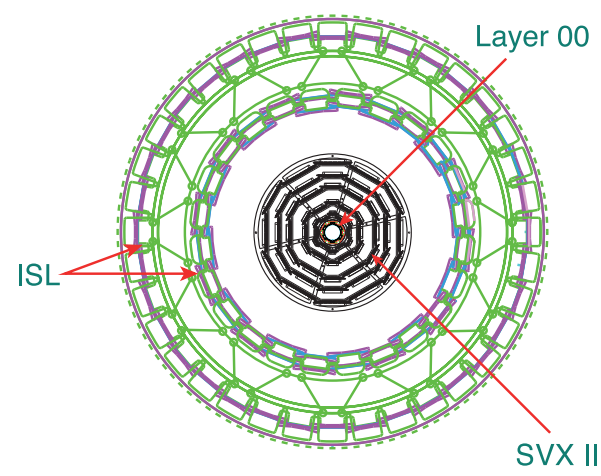

$64 \mathrm{~cm}$

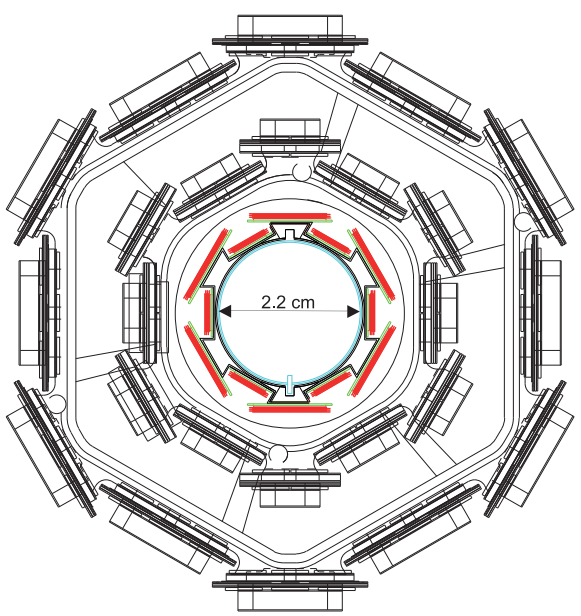

(b)

(a)

Figure 3.9. (a) End view of the complete silicon microstrip detector in $r-\phi$ plane. The outer colored structures represent the intermediate silicon layers (ISL). (b) Magnification of the inner structures, showing the Layer 00 silicon microstrip detector (green/red), built directly onto the beampipe. In addition, the first two layers of the silicon vertex detector SVX II are shown. (Drawings courtsey of the CDF collaboration).

The Layer 00. At the innermost position, directly attached to the beampipe at a radius from 1.35 to $1.62 \mathrm{~cm}$, one finds a single-sided silicon microstrip detector, called Layer 00. Not being part of the original Run II upgrade plan it was later added to improve tracking precision and tagging efficiency. The layout is shown in Figure 3.9(b) in red and green. There are overlapping wide and narrow modules consisting of end-to-end bonded silicon sensors. The wide modules have 512 strips and the narrow ones 256 strips with only half of the strips of each module being read out $^{6}$. In total Layer 00 holds 72 modules divided into three barrels with two bulkheads in the $z$ direction each built of 12 module-wedges in azimuthal angle. The Layer 00 covers a pseudorapidity region of $|\eta| \leq 4$.

The SVX II. Surrounding Layer 00 one finds the silicon vertex detector SVX II at a radius of $2.1 \mathrm{~cm}$ to $17.3 \mathrm{~cm}$ from the beamline. The detector consists of in total 360 double sided silicon microstrip sensor ladders. Those are divided into 3 barrels with

\footnotetext{
${ }^{6}$ The reason is (a) to reduce cross-talk between adjacent channels which improves the hit resolution; (b) the readout system is not capable of reading out more strips, i.e. as it was planned to add the L00 information into the trigger [91].
} 
a bulkhead on each side. Radially 5 layers ( 0 to 4 ) of 12 ladders are installed. The ladders have increasing widths towards larger radii and are overlapping with their neighbors. The two sides of a ladder hold different strip orientations (called stereo). In layer 0,1 and 3 one finds a $90^{\circ}$ stereo whereas in layer 2 and 4 a $1.2^{\circ}$ stereo. The innermost ladders hold 256 strips and the outermost 896, in the case of the axial ladder side. In Figure 3.9(a) the end view of whole SVX II detector is shown (black) and in Figure 3.9(b) magnified the first two layers (black). The SVX II covers a pseudorapidity region of $|\eta| \leq 2$.

The ISL. To improve tracking capabilities of the CDF II detector a intermediate silicon layer ISL has been installed. It helps linking of tracks from the SVX II and the COT and provides silicon only tracking in the plug region where the COT coverage is limited. The ISL consists of one central layer at a radius of $22 \mathrm{~cm}$ for $|\eta|<1$ and two layers in the plug-region at radii of $20 \mathrm{~cm}$ and $28 \mathrm{~cm}$ between $1<|\eta|<2$ on each side. In total one has 56 ladders in the central barrel split in two bulkheads each containing 28 azimuthal wedges. In the plug barrels one finds a total of 240 ladders split in the two outer barrels each with two bulkheads, each containing two layers of 12 azimuthal wedges. The inner layer holds two ladders per wedge whereas the outer layer three. As in the SVX II, doublesided modules are used with 1024 strips on the axial side and 768 on the stereo side. In Figure 3.9(a) showing the total silicon tracker cut in the $r-\phi$ plane one can see the ISL as the outer ring structure.

2.2.2. Central Outer Tracker. A cylindrical drift chamber (COT) is built outside of the silicon tracker at a radius from 40 to $137 \mathrm{~cm}$ [92]. The $310 \mathrm{~cm}$ long COT consists of 8 superlayers of driftcells starting from number 1 . A drawing of a $1 / 6$ section of the COT is shown in Figure 3.10. If one requires all superlayers for tracking it covers the pseudorapidity region of $|\eta| \leq 1$. As shown in Figure 3.11, a supercell consists of potential and signal wires including two shaper wires at both ends. Supercells are separated by a gold coated Mylar plane. The functional principle of the detector is rather simple. Both the potential and the signal wires are put under high voltage with respect to the supercell walls which generates a strong electric field. Traversing ionizing particles generate an ion avalanche in the gas-mixture the detector is filled with. The generated charge of the avalanche is collected by the sense wires resulting in a measurable signal. Each supercell is installed at an angle of $35^{\circ}$ (Lorentz-angle). The number of supercells per superlayer rise from 168 in the first up to 480 in the outermost layer. Even numbered layers hold axial wires whereas odd numbered hold wires with an stereo angle of $2^{\circ}$, which enables the COT also to measure the $z$-coordinate of hits. The COT is filled with a gas mixture of argon, 


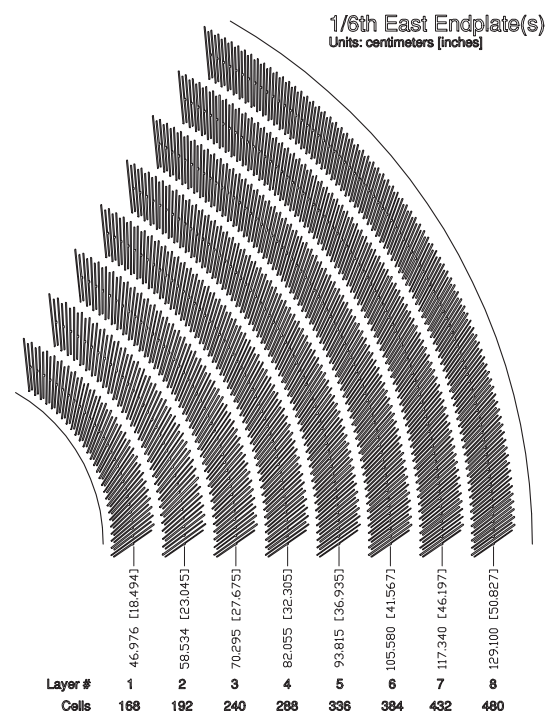

Figure 3.10. Detailed drawing of a $1 / 6$ section of the cylindrical drift chamber (COT) end plate. Detailed information is given such as the number of supercells per superlayer and the average radius. (Taken from the TDR $[85])$.

ethane and $\mathrm{CF}_{4}$ to ensure fast ion drift-times thus a faster signal response. In addition this gas mixture limits the aging of the detector.

\subsection{Calorimetry}

Apart from the tracking information of charged particles one is interested in measuring their kinematical energy. This is done by the calorimetry. So far only the momenta of charged particles from track-curvature measurements by the tracker are known. In general one wants to measure the energy of the whole event including also non-charged particles ${ }^{7}$. Opposite to the tracker, calorimeters are built out of solid heavy material with the purpose to stop high energetic particles such that they deposit all their energy. Together with the tracking information one can reconstruct the particles 4-momentum and consequently reconstruct whole events.

The CDF II calorimetry [93-95], shown in Figures 3.3 and 3.4, surrounds the tracker and the solenoid. It consists of an electromagnetic (EM) and a hadronic calorimeter, both in the central area, called the central EM calorimeter (CEM) and

\footnotetext{
${ }^{7}$ Particles such as neutrinos will most likely not deposit any energy withing the CDF detector range. These are accounted for by the missing transverse energy, MET.
} 


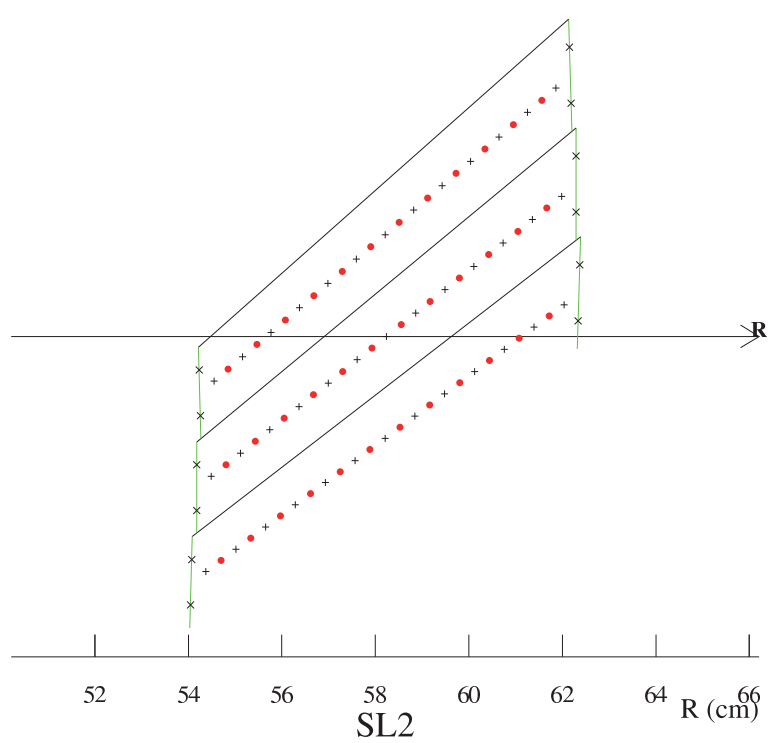

Figure 3.11. Layout of a supercell consisting of potential wires $(+)$, sense wires (red dots) and shaper wires (x) enveloped by pure Mylar sheets (green lines) and gold coated Mylar sheets as field panel (black solid lines). (Taken from the TDR $[85]$ ).

the central hadronic calorimeter (CHA), and in the plug region, called the plug EM calorimeter (PEM) and the plug hadronic calorimeter (PHA). A hadronic calorimeter called End-Wall-Hadron calorimeter (WHA) fills the gap between the central and plug hadronic calorimeters. Within the first layers of the EM calorimeter one finds strip/wire chamber detectors in the central area, called central EM shower maximum chambers (CES), central pre-radiate chambers (CPR) and central crack chambers (CCR), and in the plug region, called plug EM shower maximum chambers (PES) and plug pre-radiate chambers (PPR). Those are used for refined position measurements of EM showers. To a large extend the central calorimeter components (CEM, CHA, WHA, CES, CPR, CCR) were reused from Run I. An exception is the electronics that had been upgraded to handle higher luminosities and radiation doses.

The whole calorimetry is build out of towers with projective power spanned in $\eta$ and $\phi$. A detailed segmentation of the CDF calorimetry can be found in Table 3.1, which lists also information on the type of material and the budget used (in terms of radiation or interaction lengths) and on the energy resolution. 
TABLE 3.1. Subsystem details of the CDF II calorimetry. The symbol $\oplus$ represents sum in quadrature, $X_{0}$ the radiation length and $\lambda_{0}$ the interaction length.

\begin{tabular}{cccccc}
\hline Subsystem & Material & Energy res. & $|\eta|$ coverage & $\Delta \eta$ & $\Delta \phi$ \\
\hline $\mathrm{CEM}$ & $\mathrm{Pb} / 18 X_{0}$ & $13.5 \% / \sqrt{E_{T}} \oplus 1.5 \%$ & $0-1.1$ & 0.11 & $15^{\circ}$ \\
$\mathrm{CHA}$ & $\mathrm{Steel} / 4.7 \lambda_{0}$ & $75 \% / \sqrt{E_{T}} \oplus 3 \%$ & $0-0.9$ & 0.11 & $15^{\circ}$ \\
$\mathrm{WHA}$ & $\mathrm{Steel} / 4.5 \lambda_{0}$ & $80 \% / \sqrt{E_{T}}$ & $0.7-1.3$ & 0.11 & $15^{\circ}$ \\
$\mathrm{PEM}$ & $\mathrm{Pb} / 20.1 X_{0}$ & $16 \% / \sqrt{E} \oplus 1 \%$ & $1.1-2.1$ & $0.1-0.2$ & $7.5^{\circ}$ \\
& & & $2.1-3.6$ & $0.2-0.6$ & $15^{\circ}$ \\
$\mathrm{PHA}$ & \multirow{2}{*}{ Steel $/ 7.1 \lambda_{0}$} & $80 \% / \sqrt{E} \oplus 5 \%$ & $1.2-2.1$ & $0.1-0.2$ & $7.5^{\circ}$ \\
& & & $2.1-3.6$ & $0.2-0.6$ & $15^{\circ}$ \\
\hline
\end{tabular}

\subsubsection{The Central Calorimeter.}

The Central Electromagnetic Calorimeter. The energies of electromagnetic showers in the central detector are measured by the central electromagnetic calorimeter, which covers a pseudorapidity region of $|\eta|<1.1$. The CEM is built of alternating layers of lead and polystyrene scintillator material in a stacking mode, 18 radiation lengths ${ }^{8} X_{0}$ thick. The EM showers produced in the lead generate photons in the scintillator that are sent via wavelength shifting fibres (WLS) to photomultiplier tubes (PMT). The smallest unit of the CEM is a single projective tower spanning 0.11 units in $\eta$ and $15^{\circ}$ in azimuthal $\phi$. The towers are organized into azimuthal wedges of $15^{\circ}$ as shown in Figure 3.12. Each wedge contains 10 towers. In total there are 478 towers divided into 24 wedges per detector hemisphere. Two towers were removed to make space for cryogenic devices of the solenoid. The towers are equipped with two readout PMTs. The average energy resolution of $\sigma(E) / E=13.5 \% / \sqrt{E \sin \theta} \oplus 1.5 \%$ was measured using a $50 \mathrm{GeV} / c$ electron test beam. The CEM is equipped with a timing system for EM objects with an energy deposit $>4 \mathrm{GeV}$ that enables us to measure arrival times from the bunch crossing. For better position resolution the CEM is equipped with two proportional chambers, the shower maximum detector (CES) and the pre-radiator device (CPR).

The Central Strip and Wire Chambers. The CES is a proportional wire and strip chamber with an argon- $\mathrm{CO}_{2}$ gas mixture at shower maximum position within the CEM at 6 radiation lengths ${ }^{9}$. Its main purpose is to identify and distinguish electrons and photons and to give a good spatial resolution of approximately $2 \mathrm{~mm}$. At lower

\footnotetext{
${ }^{8}$ Radiation length $X_{0}$ is defined as the distance an EM object travels with an energy loss of $1-1 / e$ due to Bremsstrahlung.

${ }^{9}$ The $6 X_{0}$ include the tracker system of $\sim 0.11$ and the solenoid of $\sim 1 X_{0}$.
} 


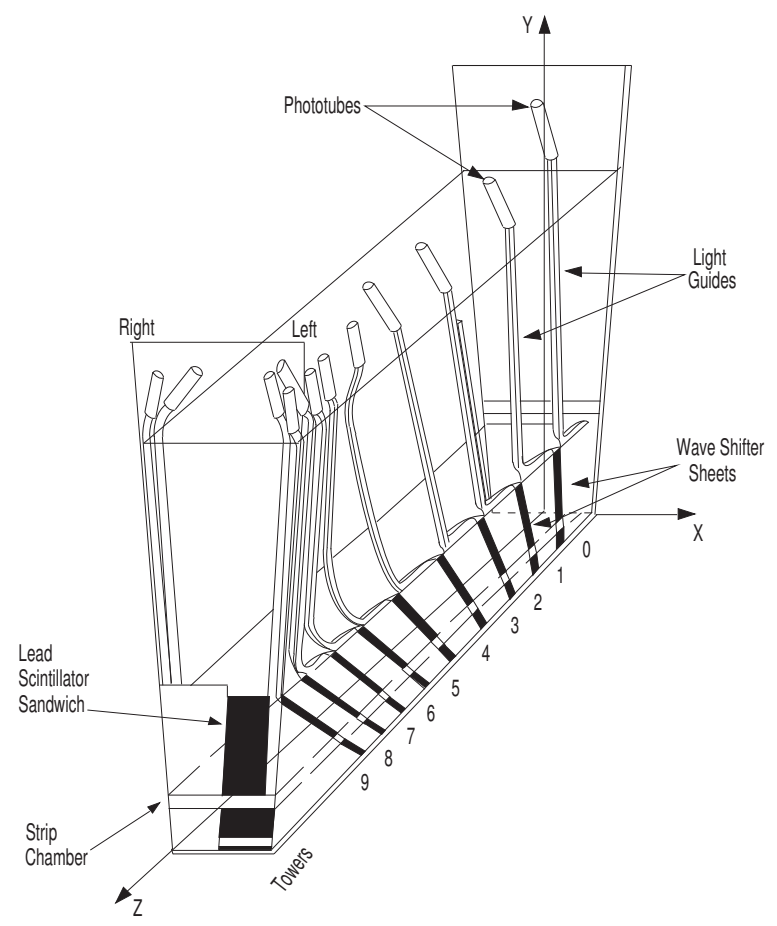

Figure 3.12. Drawing of a wedge of the central electromagnetic calorimeter. (Taken from $[\mathbf{9 3}]$ ).

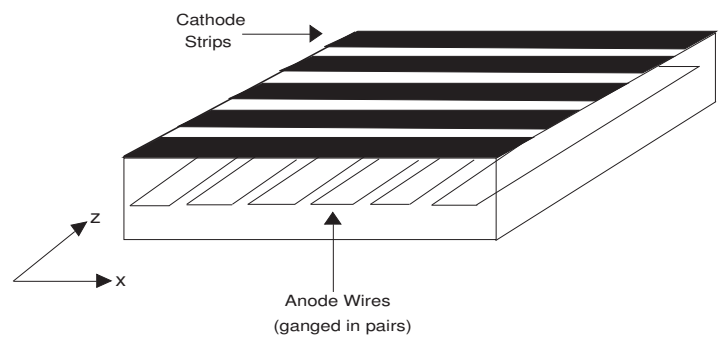

FiguRE 3.13. Schematic drawing of a wire and strip chamber detector segment embedded in the central electromagnetic calorimeter. (Drawing courtsey of the CDF collaboration).

energies the CES is also capable of distinguishing photons from $\pi^{0}$ 's. A drawing of a CES detector segment is shown in Figure 3.13. In total 48 modules are installed, one per wedge, 24 east and 24 west. Each module holds 64 anode-wires (parallel to the 
beampipe, split at $|z|=121.2 \mathrm{~cm})$ for $x$-coordinate and orthogonal 128 cathode-strips for $z$-coordinate measurements. At lower $z$ the wires span from $0.2<|z|<121.2 \mathrm{~cm}$ and at higher $z$ between $121.2<|z|<239.6 \mathrm{~cm}$. The anode-wire pitch is $1.5 \mathrm{~cm}$ and the cathode-strip pitch varies with $\eta$ from 1.7 to $2.0 \mathrm{~cm}$.

The CPR, a pre-shower detector within the first layer of the CEM, consists of multi-wire proportional chambers. By measuring the shower development at the entry of the calorimeter the photon and electron identification is greatly enhanced and it especially reduces the systematic uncertainty for direct photon tagging by a factor of three. A single CPR chamber in a wedge has in total 32 wires running along beam directions, 16 between $7.9<|z|<119.7 \mathrm{~cm}$ and 16 between $123.5<|z|<235.3 \mathrm{~cm}$. Due to its design coverage gaps between the wedges exist. Those are filled by the central crack chambers CCR), a scintillator tile device mounted on tungsten bars.

The Central Hadronic and the Wall Hadronic Calorimeters. Behind the CEM one finds the central hadronic calorimeter (CHA). The CHA has the same segmentation as the CEM with 10 towers per wedge of 0.11 units in $\eta$ and $15^{\circ}$ in azimuthal $\phi$. At higher pseudorapidity $(|\eta|>0.66)$ however the coverage is not complete due to the rectangular design of the central part of the CDF detector (see Figure 3.4). To compensate for the missing interaction length the wall hadronic calorimeter (WHA) was constructed in the gap between the central and plug calorimeter. Keeping the projective design with the combination of CHA and WHA one finds a coverage in $\eta$ of $0<|\eta|<1$. The CHA consists of 32 alternating layers of steel and scintillator. Each steal absorber layer is $2.5 \mathrm{~cm}$ thick followed by a $1 \mathrm{~cm}$ thick layer of scintillator. In the case of the WHA we find 15 layers of $5.0 \mathrm{~cm}$ thick steal with $1.0 \mathrm{~cm}$ thick scintillator layers in between. The CHA and WHA interaction length ${ }^{10}$ is around 4.5 to $4.7 \lambda_{0}$. As for the CEM two readout PMTs are connected to the scintillators via wavelength shifting fibers (WLS). The energy resolution is approximately $75 \% / \sqrt{E_{T}} \oplus 3 \%$ for the CHA and $80 \% / \sqrt{E_{T}}$ for the WHA, measured using a $50 \mathrm{GeV} / c$ pion test beam.

2.3.2. The Plug Calorimeter. The plug calorimeter, shown in Figures 3.3 and 3.4, literally looks like a plug. It seamlessly extends the calorimetry into the forward region consisting of similar functional components as the central calorimeter. Closer to the IP is the plug electromagnetic calorimeter (PEM) followed by the plug hadronic calorimeter (PHA). Within the PEM one finds a pre-radiator detector (PPR) and a shower maximum detector (PES). Similar to the central calorimeter the plug calorimeter has a projective design. One finds 48 azimuthal wedges with 8 tower

\footnotetext{
${ }^{10}$ Interaction length is defined as the mean free path length of a particles before loosing all but an energy amount of $1 / e$ due to an inelastic interaction.
} 


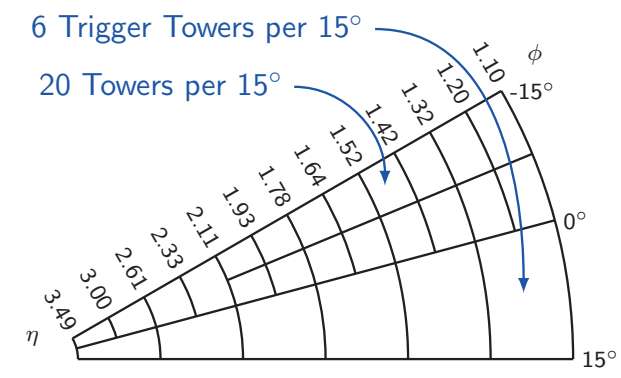

FigURE 3.14. Illustration of the azimuthal segmentation of the plug calorimeter. The top segment shows the physical towers. At trigger level towers are combined to form larger trigger towers shown in the bottom segment.

groups each covering $7.5^{\circ}$ for intermediate pseudorapidities between $1.1<|\eta|<2.1$, and 24 wedges with 4 tower groups each covering $30^{\circ}$ at more forward pseudorapidities between $2.1<|\eta|<3$.6. The segmentation is illustrated in detail in Figure 3.14. This is identical for both the electromagnetic and hadronic parts.

The Plug Electromagnetic Calorimeter. The PEM covers a pseudorapidity region of $1.1<|\eta|<3.6$. Built out of 23 alternating layers of $4.5 \mathrm{~mm}$ thick lead absorber plates and $4 \mathrm{~mm}$ thick scintillators it is equivalent to a total radiation lenght of $20.1 X_{0}$. The readout works via WLS fibers into PMTs. The PES is equipped with a timing system for EM objects $>4 \mathrm{GeV}$ up to $|\eta|=2.11$ to measure arrival times from bunch crossings. The energy resolution obtained using a $57 \mathrm{GeV} / c$ positron test beam is consistent with the design energy resolution of $16 \% / \sqrt{E} \oplus 1 \%$.

The Plug Strip and Wire Chambers. The PPR, a scintillator tile detector within the first scintillator layer of the PEM at a depth of $1.5 X_{0}$, enhances the capability of separating photons from neutral pions as well as distinguishing electrons from charged pions. The PES is a position sensitive shower maximum detector at approximately $6 X_{0}$ that consists of scintillator strips readout by WLS fibers. A schematic drawing of one sector is shown in Figure 3.15. The PES is divided into 8 sectors with two layers of 200 strips each, the $u$-layer and $v$-layer. The scintillator strip pitch is $5 \mathrm{~mm}$ and the strip orientations of the two layers are $+22.5^{\circ}$ and $-22.5^{\circ}$ with respect to the radial center, giving a crossing angle of $45^{\circ}$. This enables good position measurement resolution. A pseudorapidity region of $1.13<|\eta|<3.50$ is covered. The strips are separated into two parts at $\eta=2.6$ to reduce occupancy.

The Plug Hadronic Calorimeter. The PHA covers a pseudorapidity region of $1.2<|\eta|<3.6$. Due to limited coverage at lower pseudorapidities the WHA 


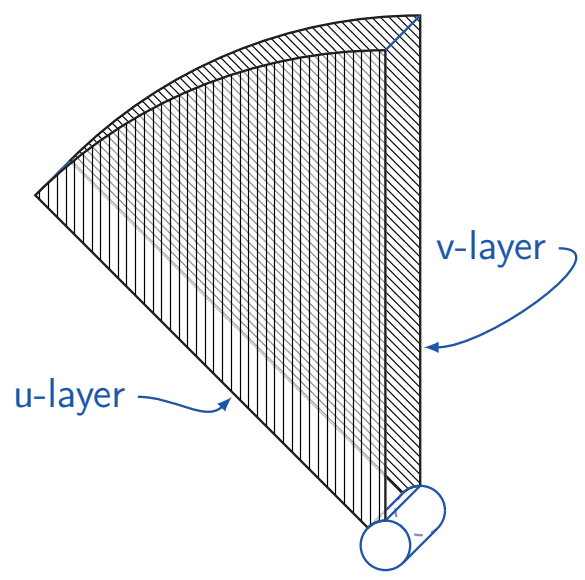

Figure 3.15. Schematic drawing of a sector of the plug shower maximum detector.

contributes to the hadronic towers in the outermost tower group. In total there are 432 PHA towers in groups of 11. Apart from that the segmentation follows the PEM. The PHA is built out of 23 alternating layers of $5.1 \mathrm{~mm}$ thick steal absorber plates and $6 \mathrm{~mm}$ thick scintillators. The total interaction length of the hadronic section is $7.1 \lambda_{0}$. The scintillators are read out with WLS fibers by PMTs. The energy resolution obtained with a pion test beam is consistent with the design energy resolution of $80 \% / \sqrt{E} \oplus 5 \%$.

\subsection{Forward Detectors}

2.4.1. The Miniplug Detector. The Miniplug calorimeter is a detector to measure both the energy and the lateral position of particles in the forward region extending the Plug calorimeters on both sides of the IP [96]. In Figure 3.6 the position of the Miniplug is shown schematically at $5.8 \mathrm{~m}$ from the IP. The detector covers the pseudorapidity region $3.6<|\eta|<5.2$ and the full azimuthal angle. The device with its towerless pixel-type geometry is a liquid scintillator type of detector that is read out by WLS fibers. Perpendicular to the 1512 fibers, which are parallel to the beampipe, are 36 lead plates installed, each $6.8 \mathrm{~mm}$ thick with holes for the fibers. The liquid scintillator is $517 \mathrm{l}$ of Bicron (mineral oil) with Pseudocumene as the active ingredient. A schematic drawing is shown in Figure 3.16. The readout structure is of hexagonal form as shown in Figure 3.17. Each lead plate consists of 252 hexagons each having 6 holes for the fibers. The six bundled fibers are read out by one channel of a 16-channel multi-anode photomultiplier tube. Three channels are combined to form one of 84 calorimeter towers, shown as shaded structures in Figure 3.17. 


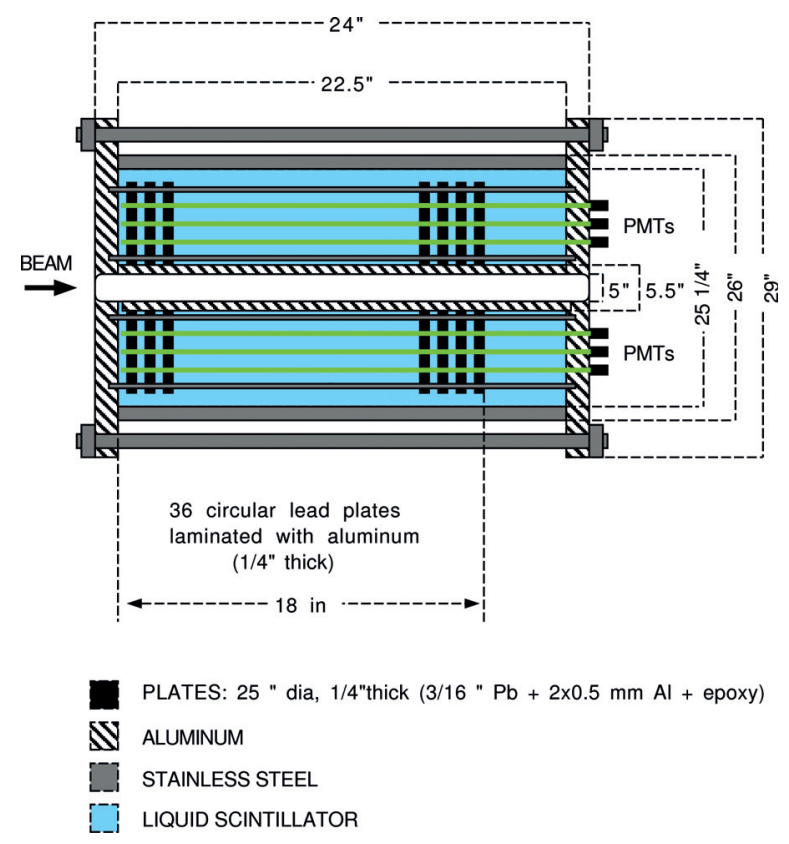

Figure 3.16. Schematic drawing of the Miniplug detector in side view. (Taken from $[\mathbf{9 6}])$.

2.4.2. Beam Shower Counters. The Beam Shower Counter (BSC) is a simple forward scintillator detector with the purpose to detect particles from the IP scattered at very small angles relative to the beampipe. There are three BSC stations installed on the proton side and four on the antiproton side, covering a pseudorapidity region of $5.4<|\eta|<7.4$ (without BSC-4). In Figure 3.6 their positions along the beampipe are shown. Details about position and coverage in pseudorapidity can be found in Table 3.2 .

The BSC devices are simple scintillator counters arranged around the beampipe. BSC- 1 consists of 4 circular counters on each station, whereas BSC-2,-3 and -4 consist only of two counters per station enclosing the beampipe, that are rectangular on the outside. In Figure 3.18 on the left the schematic drawing of half of the BSC-1 is shown including two scintillator volumes with its PMTs. In front of BSC-1 a $0.95 \mathrm{~cm}$ thick lead plate is installed to convert photons. In Figure 3.18 on the right, the schematic drawing of half of BSC-2, -3 and -4 is shown, consisting of one counter. Each scintillator has its own PMT, thus 10 signal channels from the west and 8 signal channels from the east, in total 18 channels. 


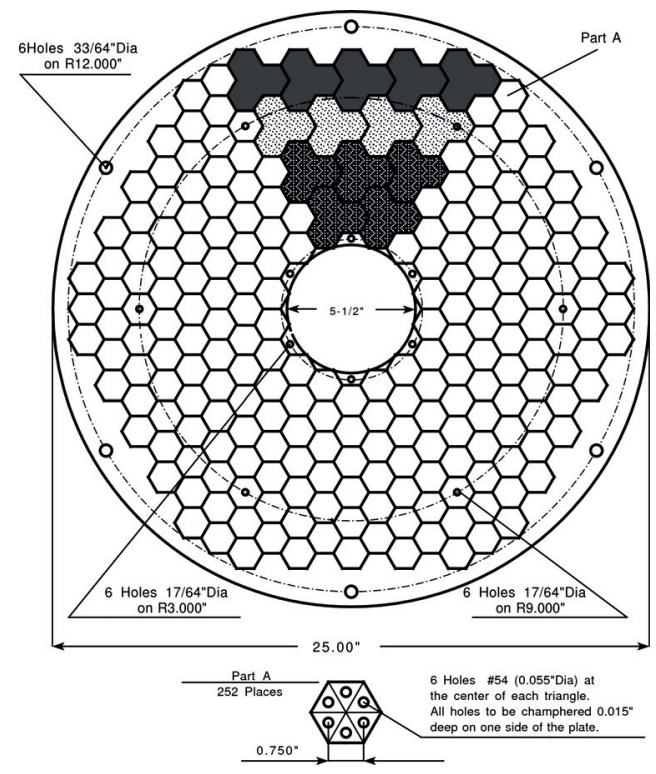

Figure 3.17. Drawing of a Miniplug lead plate with a hexagonal structure for the readout. Each hexagon has six holes for the scintillator fibres. Three hexagons are grouped to form a readout tower. (Taken from $[96])$.

TABLE 3.2. Details of the beam shower counters installed in CDF II. Note that the sensitive volume of BSC-2, -3 and -4 are squared on the outer side which increases the coverage in $\eta$ slightly. The numbers given only show the minimal outer $\eta$ bound.

\begin{tabular}{lccc}
\hline Station & Segments & $z$ position $(\mathrm{m})$ & $|\eta|$ coverage \\
\hline BSC-1 west & 4 & 6.6 & $5.4<|\eta|<5.9$ \\
BSC-1 east & 4 & -6.6 & $5.4<|\eta|<5.9$ \\
BSC-2 west & 2 & 23.2 & $6.4<|\eta|<7.1$ \\
BSC-2 east & 2 & -23.2 & $6.4<|\eta|<7.1$ \\
BSC-3 west & 2 & 31.6 & $6.7<|\eta|<7.4$ \\
BSC-3 east & 2 & -31.6 & $6.7<|\eta|<7.4$ \\
BSC-4 west & 2 & 56.4 & $7.3<|\eta|<8.0$ \\
\hline
\end{tabular}

The BSCs are sensitive both to prompt particles and secondary particles. The main purpose of those devices is to study diffractive physics acting as veto counters. BSC-1 in addition was also used for studying beam losses of the Tevatron. 


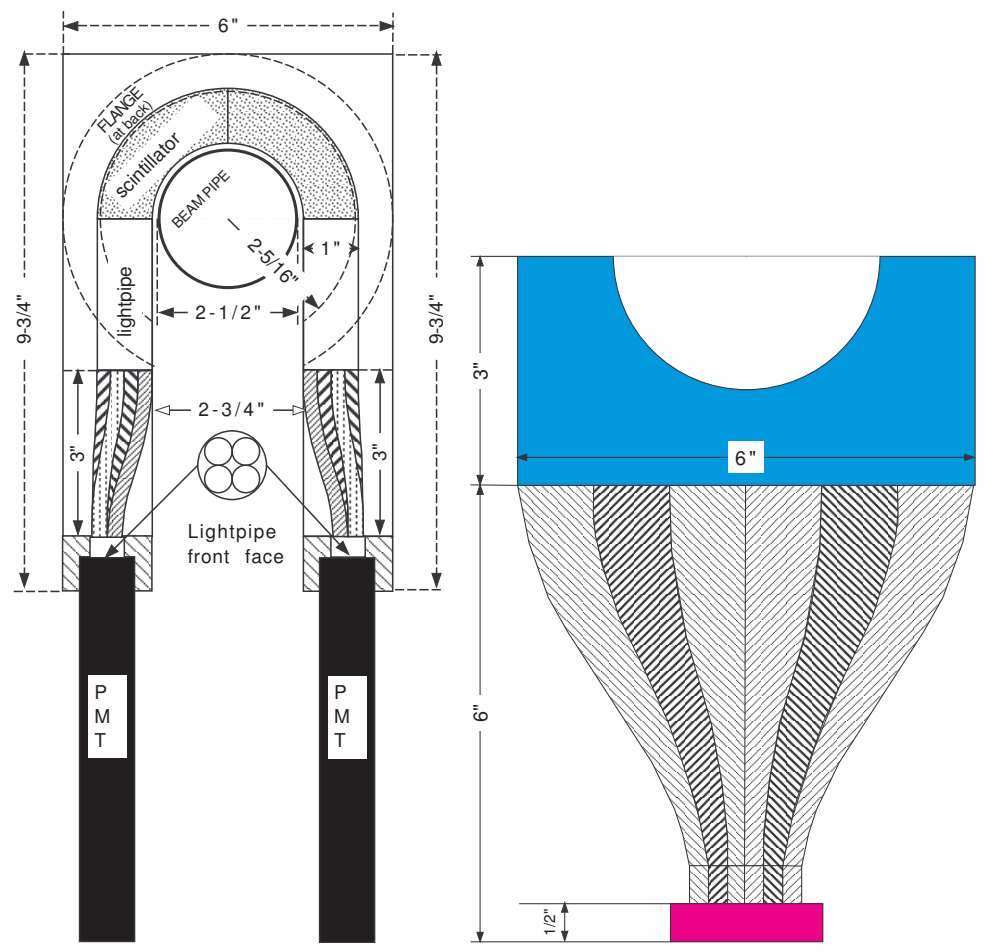

Figure 3.18. Drawing of the beam shower counters; (left) design of BSC-1 and (right) design of BSC-2, -3 and -4 . (Drawings courtsey of the CDF collaboration).

2.4.3. Cherenkov Luminosity Chambers. With the purpose of accurate online luminosity measurements, a Cherenkov Luminosity Counter (CLC) was introduced in Run II $[\mathbf{9 7}, \mathbf{9 8}]$. The Detector is a gaseous Cherenkov light detector built around the beampipe in the gap between the Plug calorimeter and the beampipe itself. The detector that was also used to monitor Tevatron's performance covers an pseudorapidity region of $3.7<|\eta|<4.7$. One module is built out of three circular layers each containing 16 counters, in total 48 counters each pointing towards the IP (see Figure 3.19). The counters have a length of $180 \mathrm{~cm}$ installed in the two outer layers and a length of $110 \mathrm{~cm}$ in the inner layer. The counters themselves consist of cylindrical tubes with diameters between 2 and $6 \mathrm{~cm}$, filled with isobutane gas at atmospheric pressure. The gas offers a large refractive index. The tubes are made out of reflective aluminized Mylar rolled into a conical shape. At the end of the tubes (pointing away from the IP) a conical mirror is installed that concentrates the collected Cherenkov 

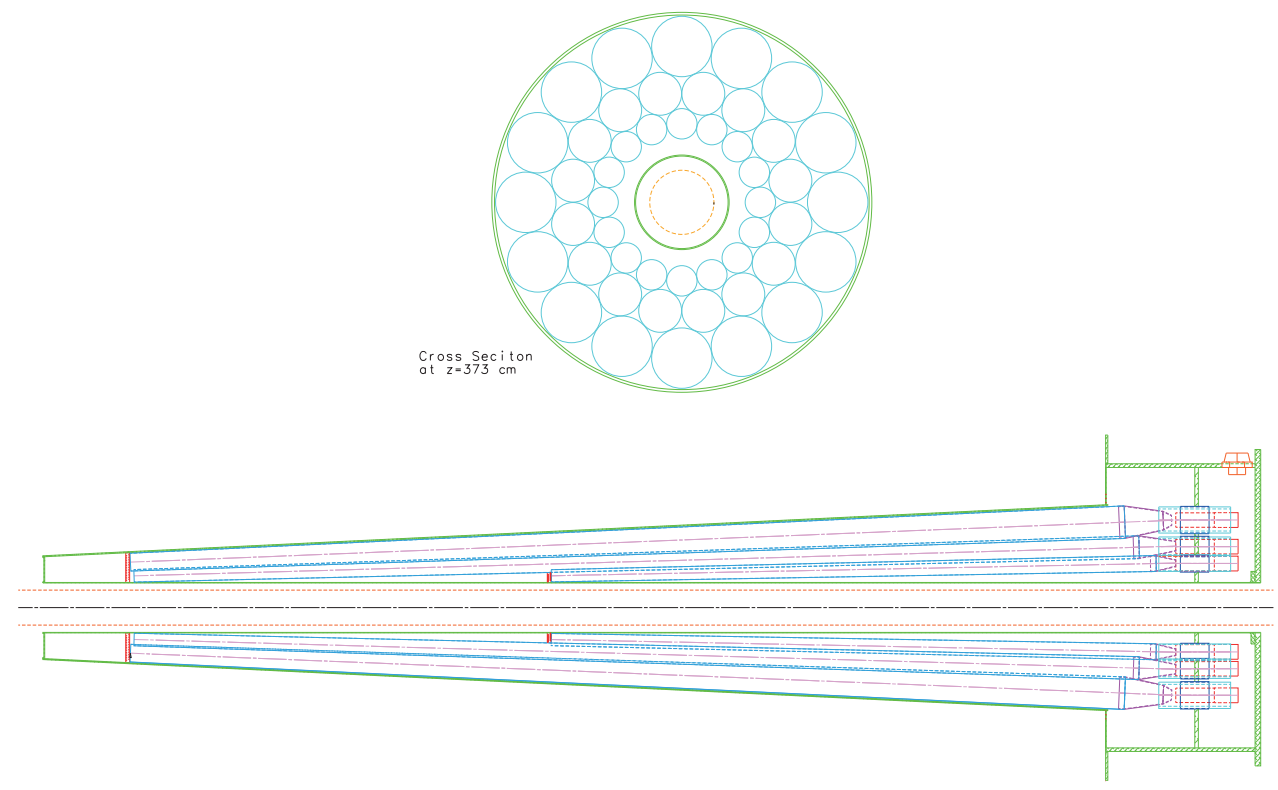

Figure 3.19. Drawing of the Cherenkov Luminosity Counter (CLC) device. (Taken from $[\mathbf{9 7}]$ ).

light towards the photomultiplier tubes (PMT). Fast PMTs allow a timing resolution of $<100$ ps.

Thanks to its projective design, the CLC has efficient background rejection capability. Primary particles produced by $p \bar{p}$ collisions are more likely to traverse a large volume of the CLC tubes than secondary and lower energetic particles produced close to the CLC. Secondary particles traverse the detector more likely with larger angles (thus cross smaller volumes) resulting in a smaller signals. Secondary background particles are mostly rejected by requiring a minimal light yield threshold.

The total light yield per module is used to estimate the average number of particles that pass the detector. This enables us to estimate the luminosity of the Tevatron beam at CDF interaction point using the following formula,

$$
\mathcal{L}=\frac{f}{\sigma_{\text {inel }} \cdot \varepsilon_{\alpha}} \cdot \frac{\left\langle N_{H}\right\rangle_{\alpha}}{\left\langle N_{H}^{1}\right\rangle_{\alpha}},
$$

where $f$ is the bunch crossing frequency, $\sigma_{\text {inel }}$ the inelastic cross section for $p \bar{p}$ at $\sqrt{s}=1.96 \mathrm{TeV}, \varepsilon_{\alpha}$ the acceptance for a single $p \bar{p}$ interaction, $\left\langle N_{H}\right\rangle_{\alpha}$ the average number of hits in the CLC per bunch crossing and $\left\langle N_{H}^{1}\right\rangle_{\alpha}$ the average number of hits in the CLC for a single $p \bar{p}$ interaction. The luminosity measurement method using 
the CLC yields a total uncertainty of $\sim 6 \%$. This is composed of an CLC acceptance uncertainty of $<4.2 \%$ and an uncertainty on the inelastic cross section of $\sim 4 \%$.

2.4.4. The Roman Pot Spectrometers. The Roman $\operatorname{Pot}^{11}$ spectrometers (RPS) are scintillator fibre type detectors installed along the beampipe on the antiproton side around $57 \mathrm{~m}$ downstream from the IP. The purpose of those devices is to measure small angle scattered antiprotons. The detectors themselves are positioned inside the beampipe, hosted in so-called Roman Pots. In total three station are installed to enable precise antiproton tagging. Each station supplies 40 readout channels (fibers) in the $x$-direction and 40 in the $y$-direction. The RPSs are during setup of the beam in their parking positions. Only after the beam is set up and beamlosses are under control the RPSs can be moved to their final position approximately $10 \mathrm{~mm}$ from the beam.

\subsection{Additional Detection Devices}

2.5.1. Time-of-Flight Detector. The time-of-flight detector (TOF) consists of 216 scintillator bars each $3 \mathrm{~m}$ long, outside the COT, built with a barrel shape design. Using read out via lightguides by fine-mesh PMT's, the TOF enhances charged kaon identification, B meson flavor determination and helps rejecting cosmic background among others.

2.5.2. Muon Chambers. The outermost layer of CDF II consists of muon chambers. Muons are minimum ionizing particles (MIP) that leave tracks in the tracking system but do not deposit all energy in the calorimeters. In fact muons will pass through the whole detector and therefore muon chambers are placed as the last instance outside the calorimetry volume. The chambers are shielded by steel plates to prevent charged pions escaping the hadronic calorimeter and falsely being detected as muons. Muon chambers at CDF are drift tubes and to some extent scintillation counters. The whole muon system is divided into several subsystems depending on the location and device type. The whole muon system coverage in $\eta$ and $\phi$ can be seen in Figure 3.20.

In the central area one finds the original central muon detector (CMU), a sense wire drift chamber filled with a gas-alcohol mixture. In total 144 modules of 4 cells are installed covering $|\eta| \lesssim 6$.

This region is also covered by the central muon/scintillator upgrade (CMP/CSP), a 4 layer drift tube devive with a single rectangular scintillator layer on the top.

\footnotetext{
${ }^{11}$ The devices are named after the CERN-Rome collaboration who build for the first time such detectors inside the vacuum chamber of the beampipe of the ISR experiment.
} 


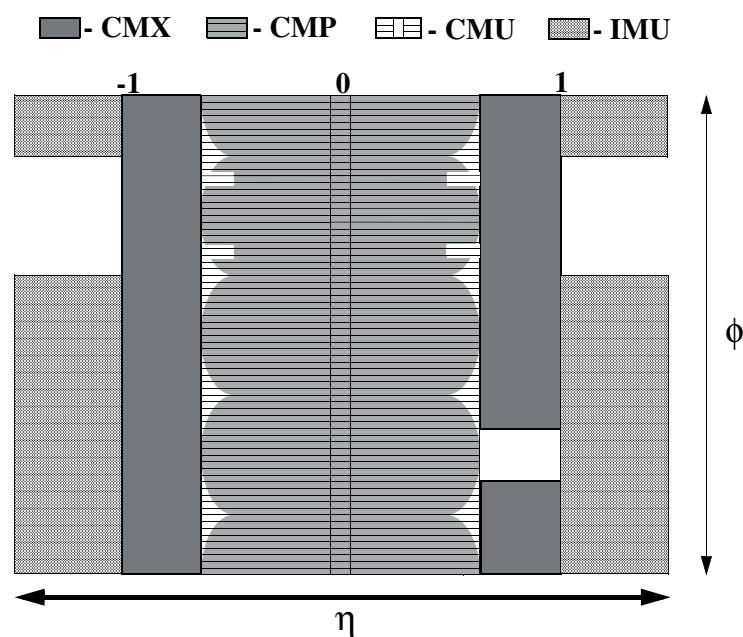

Figure 3.20. Coverage of the CDF Run 2 muon systems in $\eta$ and $\phi$. (Taken from the TDR [85]).

The coverage in $|\eta|$ is extended up to 1 by the central muon/scintillator extension (CMX/CSX), consisting of several layers of drift tubes with canonical geometry and a scintillator device on the outside.

Furthermore, several other devices such as the intermediate muon detectors (IMU) extend the muon coverage beyond a $|\eta|$ of 1 . Some devices, mostly of a scintillator type can be used by the online trigger system, otherwise for offline reconstruction.

\subsection{Trigger}

In the search for rare interesting physics at collider experiments, a fast and efficient online event selection system (trigger) is needed. Collisions at Tevatron occur with a rate of $7.6 \mathrm{MHz}$, simply too many to be recorded by the data acquisition system (DAQ). Besides that, not all collisions deliver interesting physics events. In fact mostly one is searching for very rare physics events in a huge amount of minimum-bias events ${ }^{12}$, in other words one is looking for a needle in a haystack. This is why an efficient filter is needed, a system that enables us to extract only the more likely interesting events for later offline studies. W-boson production rate, as an example, is around 6 orders of magnitude smaller than the rate of generating minimum-bias events. The filter installed at CDF Run II is a 3-level deadtimeless trigger system that reduces the event rate to approximately $100 \mathrm{~Hz}$, an acceptable level for the DAQ. The first

\footnotetext{
${ }^{12}$ The name minimum-bias is commonly used for events of soft (low- $p_{T}$ ) inelastic interactions.
} 

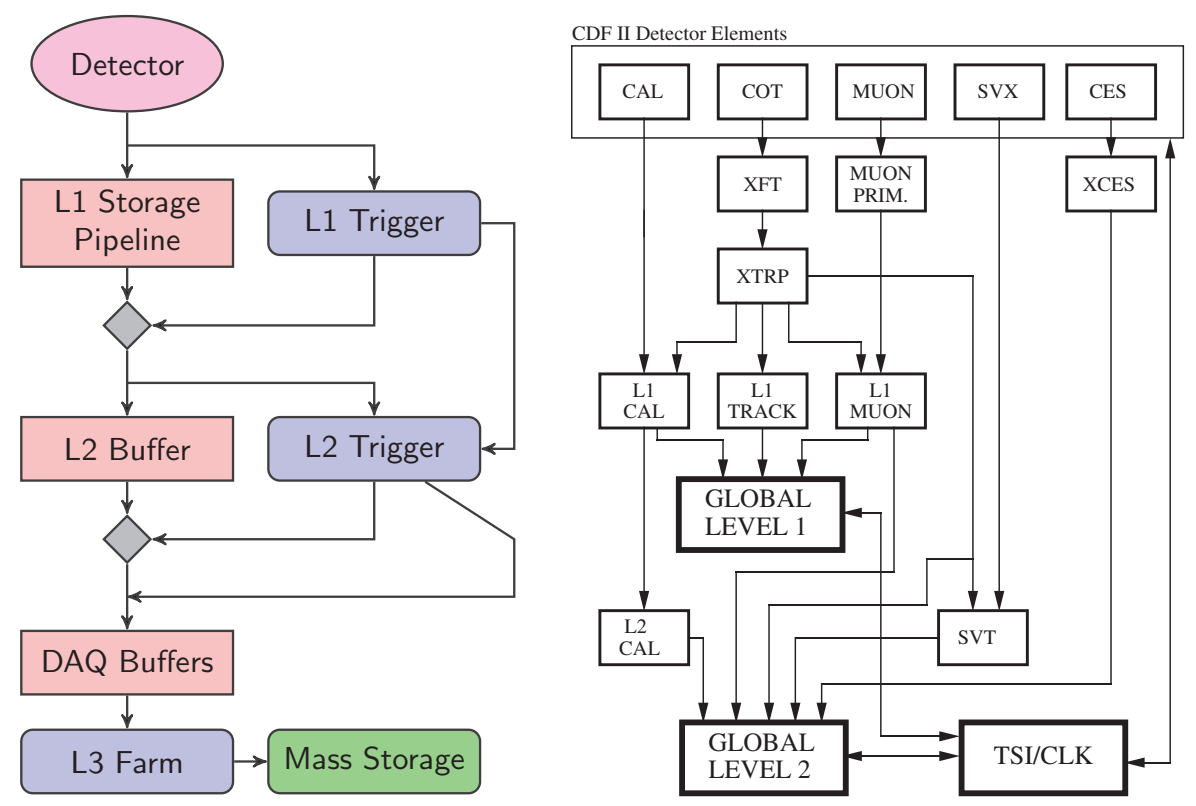

Figure 3.21. Data flow diagram of the CDF Run II trigger system (left) and block diagram of the Level 1 and Level 2 trigger system (right). (Diagram on the right taken from the TDR [85]).

and second trigger level are custom hardware based offering fast decisions using basic detector information and rough and simple pattern recognition. The third level is based on CPU farms with full online reconstruction capabilities. A schematic flow chart diagram of the CDF Run II trigger system is shown in Figure 3.21 on the left.

2.6.1. Level 1. The first trigger level is a hardware designed and programmed system. It uses low level information such as energy deposited in calorimeter towers, hits in the muon chambers and, new for Run II, COT-tracks. Those three Level 1 streams can be logically combined (AND, OR) to generate up to 64 Level 1 triggers. A detailed block diagram including Level 1 information is shown in Figure 3.21 on the right. Missing from the block diagram are BSC, CLC and TOF components that substitute information to the Level 1 trigger. The trigger system is synchronized with the Tevatron master clock with a period of 132 ns. Event data from a bunch crossing each $396 \mathrm{~ns}$ is sent to the Level 1 storage pipeline which is 42 clock cycles deep. That gives the Level 1 system maximally $5.544 \mu$ s decision time (L1 latency) before the event is lost, which minimizes the overall deadtime. The average overall Level 1 trigger acceptance rate was $<20 \mathrm{kHz}$. 
2.6.2. Level 2. The second trigger level as well uses a hardware programmed asynchronous system to reduce the event rate by a factor of $\sim 100$ down to about 100 to $300 \mathrm{~Hz}$, the overall Level 2 acceptance rate. Four event buffers accept events that passed Level 1 requirements. The events are kept in the buffer until a Level 2 decision is made. This differs from the buffer of the Level 1 system. It causes deadtime if all buffers are occupied and new events from Level 1 are accepted. However, with a designed Level 2 latency of $20 \mu$ s deadtime remains low. As shown in the block diagram in Figure 3.21 on the right, Level 2 can process information from the shower maximum detectors and, for the first time at a hadron collider experiment, from the silicon vertex detector. Starting from the already available COT tracks, the tracking resolution using hits in the silicon detector increases massively. This enables triggering on secondary vertices ${ }^{13}$. Also, calorimeter clusters are reconstructed with a improved resolution that enables jet reconstruction at trigger level.

2.6.3. Level 3. Two main components characterize the Level 3 trigger system, the event builder and the Level 3 Farm. All information from detector readout subsystems after Level 2 acceptance are assembled to a complete event by the event builder. From there the event is sent to the Level 3 processing farm for further higher level reconstruction. The processing or filter farm consists of 18 subfarms with 20 nodes each. Each node contains a dual CPU for the reconstruction of a full single event. In the CPU farms event details such as full 3D track reconstruction can be processed online in real-time. Approximately every fourth event will pass Level 3 trigger criteria. This makes an event rate of maximally $75 \mathrm{~Hz}$ being written onto storage tapes. Actually the maximal event rate is limited by bandwidth of the storage system of $15 \mathrm{Mb} / \mathrm{s}$ at that time. An average event has the size of about $200 \mathrm{~kb}$. Events on tape are then available for full offline reconstruction and analysis.

\subsection{Data Quality and Reconstruction}

The file format for the event data is provided by ROOT, an object oriented data analysis framework [109], in the form of a binary format optimized for size and fast data access. Data does not flow continuously as the luminosity of the colliding protonantiproton bunches in the Tevatron decrease exponentially. Periodically the remaining hadrons in the storage ring are dumped and replaced by a new filling (store). The data therefore is naturally split into different runs that last maximally the lifetime of a Tevatron store ( $\sim 18$ hours without incident). Before a physics data-taking run starts all subdetectors undergo a calibration procedure. During a run data is carefully

\footnotetext{
${ }^{13} \mathrm{~A}$ secondary vertex is a vertex shifted from the primary vertex.
} 
monitored online by shift personnel. Good Run bits are set for all subdetectors and the data acquisition system (DAQ) as part of the quality monitoring system at CDF. At the end of a run a detailed run summary is given and relevant information is added to the run database.

The raw data is now reconditioned for later physics analyses. This means that the data is processed offline in several steps in the times after data taking, starting with the offline data calibration that is followed by the reconstruction of high level physics objects and the ntupling into a handy format for the usage in the final physics analyses framework. The data handling is performed by the SAM-grid system (Sequential data Access via Meta-data [110]). Next, some more details are discussed about the key procedures in the data production.

Good Run Bits. Data quality monitoring is essential for obtaining reliable data used in the search for new physics. At CDF the online shift crew with the help of subsystem experts are responsible for setting the Good Run bits for each subsystem (subdetectors, DAQ) for a physics run. Table 3.3 shows relevant Good Run bits required for the analysis discussed in this work. In the case where core components

TABLE 3.3. Good Run bits set to true as required for this analysis.

\begin{tabular}{|c|c|c|}
\hline online core bits & $\begin{array}{l}\text { Good Run bits } \\
\text { additional online bits }\end{array}$ & additional offline bits \\
\hline $\begin{array}{c}\text { RUNCONTROL_STATUS } \\
\text { SHIFTCREW_STATUS } \\
\text { CLC_STATUS } \\
\text { L1T_STATUS } \\
\text { L2T_STATUS } \\
\text { L3T_STATUS } \\
\text { COT_STATUS } \\
\text { CCAL_STATUS } \\
\text { PCAL_STATUS } \\
\text { CSMX_STATUS }\end{array}$ & $\begin{array}{c}\text { PSMX_STATUS } \\
\text { MNP_STATUS } \\
\text { BSC_STATUS }\end{array}$ & $\begin{array}{c}\text { CCAL_STATUS } \\
\text { PCAL_STATUS } \\
\text { COT_STATUS }\end{array}$ \\
\hline
\end{tabular}

are marked as good the overall Good Run status will be set to true. More details can be found in reference $[\mathbf{1 1 1}]$.

Calibrations. Essential for the reconstruction of the physical quantities of events (see list above) a good calibration system is needed. A large number of parameters need to be set to correct the data such as the beam position, the detector alignment, $d E / d x$, timing, etc. Special calibration runs are performed for each subdetector right 
before a physical data-taking run starts and saved as separate datastreams. One divides between online and offline calibrations. Online calibrations such as the timing and $d E / d x$ correction to the COT are applied at trigger level [112]. Calibrations performed at the reconstruction level, i.e. after the events passed the trigger system, are called offline. The offline energy calibration of the CDF calorimeter for example uses two calibration factors $\mathrm{LER}_{\text {offline }}$ and $\mathrm{SCL}_{\text {offline }}$,

$$
E_{\text {final }}=E_{\text {online }} \times \mathrm{LER}_{\text {offline }} \times \mathrm{SCL}_{\text {offline }}
$$

Here, $\mathrm{LER}_{\text {offline }}$ represents the tower-by-tower correction factor determined by measuring the $E / p$ of electrons. For some calorimeter subdetectors, the time dependent energy scale factor $\mathrm{SCL}_{\text {offline }}$ is obtained by, among others, minimum bias data or muons from $J / \psi$ decays [113]. More detailed information about calibrations for the various CDF subsystems can be found in the CDF internal notes and the technical design report $[85]$.

Reconstruction. The event reconstruction is software based and executed on a large PC computing farm. Event reconstruction means finding high level physics objects such as charged particle tracks, type of particles, jets, missing transverse energy (MET), Phoenix electrons, etc., within the raw data-streams from the detector. The executable program ProductionExe is part of the CDF software framework AC++ and coordinates the reconstruction of all physics objects based on special offline reconstruction software classes. The software version used in this work was 6.1.4. After the reconstruction only higher level objects are kept in the events and raw detector information is removed. This reduces the file size substantially. In case some information is missing one has to go back to the raw event format level (data summary tier, DST) and reprocess the data to add extra objects. 



\section{CHAPTER 4}

\section{Short Review of CEP Searches at Hadron Colliders}

This chapter should give the reader a non complete overview of major results on the search of high energy CEP processes at hadron colliders prior to the successful search for exclusive diphoton production at the Tevatron. First a short look at the Intersecting Storage Rings (ISR) and the CERN proton-antiproton collider (Sp $\bar{p} S$ ) is given followed by more recent results from the CDF experiment at the Tevatron.

\section{Early CEP Searches}

The ISR, the worlds first proton-proton collider, that started in the year 1971 provided proton-proton collisions at various energies from $\sqrt{s}=31$ up to $63 \mathrm{GeV}$. The first collaboration to publish evidence for DIPE processes was ARCGM [99]. Their detector had full angular coverage using scintillator counters and was able to measure forward outgoing protons. Events with leading protons on each side in coincidence with two charged particles in the central detector with a maximal rapidity of $|y|=1.5$ had been measured for different energies. The measured cross section of $\sigma_{\mathrm{DPE}} \approx 20 \mu \mathrm{b}$ is consistent with phenomenological Regge calculations. Other collaborations such as CCHK and CHOV also studied DIPE mediated processes, mostly $p+p \rightarrow p+\pi^{+} \pi^{-}+p[4]$. In addition using different detector designs particle momenta could be measured including those of the surviving protons. The CCHK collaboration for the first time required 2 rapidity gaps $\Delta y>3$ on each side of the central system. The early ISR experiments overall had rather poor particle detection capabilities which led to low statistics. This improved substantially with the advent of the Axial Field Spectrometer (AFS).

The AFS collaboration studied central exclusive production of mesons, mainly charged pions but also kaons and proton-antiproton pairs [100]. The reference also includes a short description of the detector. In Figure 4.1 the invariant mass spectrum of the central charged pion pair system is shown recorded at an energy of $\sqrt{s}=63 \mathrm{GeV}$. The full mass distribution opened a window to understand earlier obtained ISR results as well as to new physics that is still after more than 30 years not fully understood. 


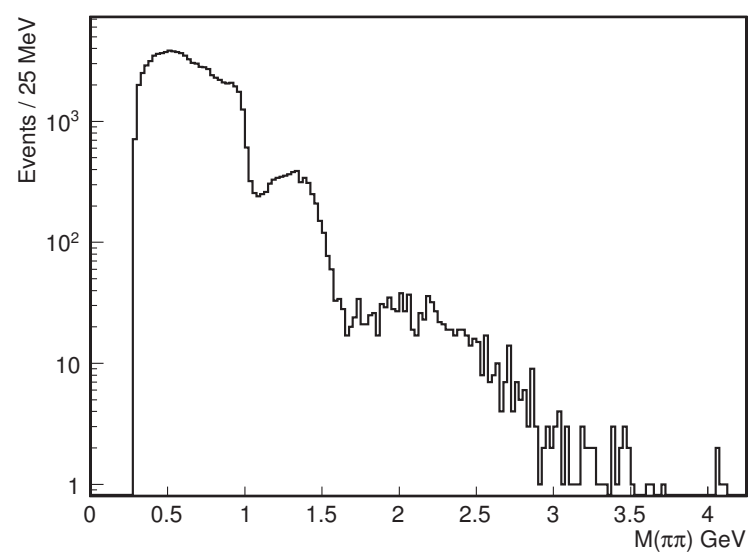

FigURE 4.1. Invariant $\pi^{+} \pi^{-}$mass distribution measured by the AFS collaboration at ISR. Figure reproduced from [100].

The spectrum raises first steeply towards a plateau around $500 \mathrm{MeV}$, then has a drastic drop around $1 \mathrm{GeV}$ followed by another drastic drop around $1.5 \mathrm{GeV}$. Some structure is also seen above $2 \mathrm{GeV}$. By partial wave analysis some secrets of this spectrum have been answered. The absence of a $\rho^{0}$ peak around $770 \mathrm{MeV}$ shows the strict spin selection rules of DIPE processes which forbids such $J^{P C}=1^{--}$states. Exclusive photo-production allows the production of such states but not in the kinematical region of the selected forward protons at the AFS. One possible interpretation of the above spectrum is a very broad $f_{0}(600)$ peak that could represent a scalar glueball in a $0^{++}$ state. The production of the $f_{0}(980)$ and $f_{0}(1500)$, both $0^{++}$states, are seen in form of the rigorous drops due to destructive interference effects [101]. Future experiments are needed to explain the true nature of this low mass scan of the ISR collisions. It is worthwhile to mention that also ABCDHW collaboration published interesting results from data collected by the Split Field Magnet spectrometer (SFM) $[\mathbf{1 0 2}, \mathbf{1 0 3}]$. They selected $p p \rightarrow p p \pi^{+} \pi^{-}$events at a center of mass energy of $\sqrt{s}=62 \mathrm{GeV}$ with two leading protons at large Feynman-x $x_{F}>0.9$. The results obtained are comparable to the results from the AFS experiment [100]. Due to the different allowed 4-momentum region for the protons, the mass distributions show a different shape. Clearly evidence for a possible $f_{0}(1270)$ peak was seen and a rapid drop at $1 \mathrm{GeV}$ [102]. Later a refined analysis that used less restricted cuts on $x_{F}>0.7$ for the forward protons gained more statistics and a partial wave analysis was done. Evidence for the S-wave scalar mesons $f_{0}(975)$ and $f_{0}(1400)$ and the D-wave meson $f_{2}(1270)$ was shown. As the 
production mechanism of the latter could be two-gluon exchange a glueball structure can be imagined $[\mathbf{1 0 3}]$.

However, one big obstacle is that the data was collected at low $\sqrt{s}$ which has the consequence that the two rapidity gaps on each side could not be larger than $\Delta y=3$. This caused large non DIPE background with other quantum numbers, e.g. the $\rho^{0}$ with $I^{G} J^{P C}=1^{+} 1^{--}$.

After ISR some limited results have been obtained at the $\mathrm{Sp} \overline{\mathbf{p}} \mathrm{S}$. At the IP of the UA2 experiment, Roman pot detectors had been installed on both sides (UA8 experiment [104]). Around 100 events with tracks in both Roman pots $\left(x_{F}>0.95\right)$ and rapidity gaps on both sides of a central system $(\Delta \eta \gtrsim 3)$ could be extracted with central masses $M_{X}<10 \mathrm{GeV}$. Momentum measurements of the particles in the central apparatus was not possible due to the absence of a magnetic field. Mass reconstruction based on the calorimetry alone made it impossible to determine the type of the central systems and to give further information about resonant structures. However, the cross section for DIPE processes, $\sigma_{\mathbb{P P}}$, was measured over the mass range of the central system showing some disagreement with simple Regge based models as given by Equation (77).

UA1 also studied DIPE physics. Not having Roman-pot devices UA1 used their calorimetry devices with a coverage up to $|\eta|=6$ to look for events with large rapidity gaps on both sides of a central cluster. The allowed mass range is somewhat larger compared to the UA8 experiment with $10<M_{X}<70 \mathrm{GeV}$. The focus was mainly on multiplicity studies comparing the extracted diffractive events to minimum bias data $[\mathbf{1 0 5}]$.

\section{CEP Searches at the Tevatron}

At the Run II Collider Detector at Fermilab (CDF II) central exclusive production (CEP) has been studied extensively with great success, even though a proposal to look for an exclusively produced Higgs boson using forward tracking devices was not accepted [5]. Instead the large rapidity coverage of the CDF calorimetry including the scintillator devices along the beampipe on both sides have been used to extract exclusive events. In particular the QED production of $e^{+} e^{-}$and $\mu^{+} \mu^{-}$was observed and a search for the Photoproduction of the $Z$ boson and the $J / \Psi$ meson was performed that concluded in an observation of the latter. DIPE processes such as the production of exclusive dijets, charmonium and $\chi_{c}$ were observed. In addition and prior to the analysis discussed in this thesis, evidence for the CEP of diphotons in hadron-hadron collisions had been found. 


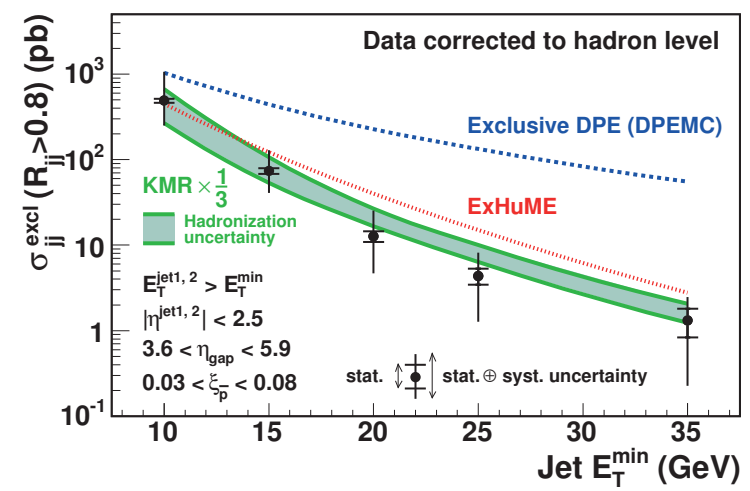

Figure 4.2. Exclusive dijet cross sections compared to Monte Carlo and LO analytical predictions.

\subsection{Exclusive Dijet Production}

Exclusive dijets had been observed in CDF II data [106]. They had been extracted from data of $310 \mathrm{pb}^{-1}$ integrated luminosity using the ratio of the dijet mass to the total mass $R_{j j}=M_{j j} / M_{x}$ of the final state. At high $R_{j j}>0.8$ exclusive dijets can be separated from inclusive diffractive dijets. Antiprotons tagged by the RPS ensure a good separation of diffractive and non-diffractive events. The measured cross sections (see Figure 4.2) clearly favor the KMR-based predictions (ExHUME or the KMR analytical calculation) $[8,107]$.

\subsection{Exclusive Electron-Positron Pair Production}

By introducing a special trigger requiring two EM showers in the calorimeter and a void of particles in the forward using BSC-1, the QED production of exclusive $e^{+} e^{-}$via virtual radiated photons was observed for the first time in data of $532 \mathrm{pb}^{-1}$ integrated luminosity in $p \bar{p}$ collisions [10]. A special method to ensure exclusiveness was used. The calorimetry including the forward detectors are divided into sections. For each section the noise level was defined. After subtracting the signal each event was filtered for additional activity above noise within $|\eta|<7.4$. In total 16 exclusive $e^{+} e^{-}$candidates with $E_{T}>5 \mathrm{GeV}$ and $|\eta|<2$ were found. The measured cross section of $\sigma=1.6_{-0.3}^{+0.5}$ (stat) \pm 0.3 (syst) $\mathrm{pb}$ is in good agreement with theoretical predictions.

\subsection{Exclusive Charmonium and Muon Pair Production}

In parallel the CDF collaboration had started to look at exclusive dimuon states [82]. With a special trigger for central muons and forward gaps, 402 back to back $\mu^{+} \mu^{-}$ 


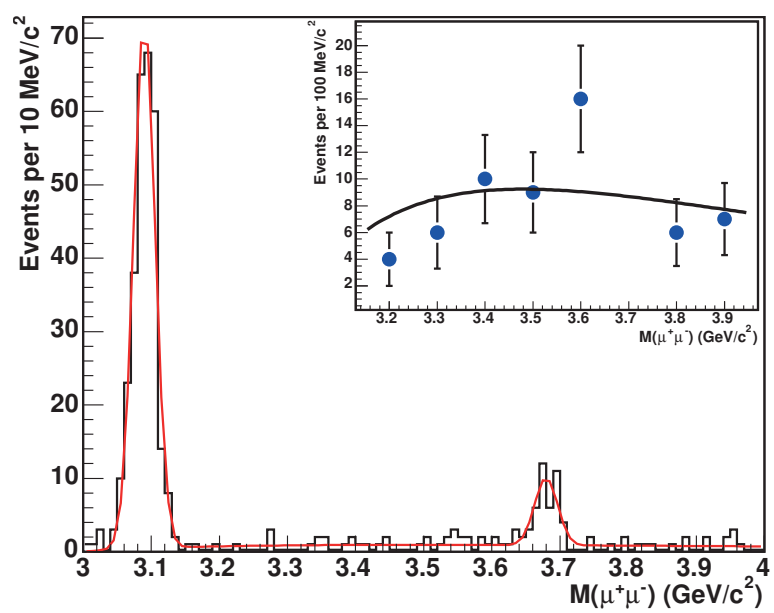

Figure 4.3. Invariant exclusive dimuon mass distribution with two Gaussian fits of the $J / \Psi$ and $\Psi(2 s)$ peak. The inlay graphic shows the exclusive dimuon QED continuum with the $\Psi(2 s)$ peak excluded.

candidates with $M_{\mu \mu}$ between 3 and $4 \mathrm{GeV}$ and $|\eta|<0.6$ were extracted from $p \bar{p}$ collisions of $1.48 \mathrm{fb}^{-1}$ integrated luminosity. The invariant mass distribution is shown in Figure 4.3. Clearly seen are the $J / \Psi$ and $\Psi(2 s)$ peaks and the QED continuum in the inlay graphic. The first observations of the exclusive photoproduction of charmonium and the exclusive QED production of $\mu^{+} \mu^{-}$in hadron-hadron collisions are also in good agreement with theoretical predictions. Allowing an additional photon in the final state, an increase of 66 events in the $J / \Psi$ peak were found compared to only one event in the $\Psi(2 s)$ peak. This is clear evidence for exclusive $\chi_{c}$ production via DIPE, decaying to a $J / \Psi$ plus a photon.

\subsection{Search for Exclusive $Z$ Production}

The collaboration also searched for exclusive photoproduction of the $Z$ boson [108]. Due to its small cross section this process was not expected to be found at the Tevatron. Any sign would have been an indication for beyond the standard model physics. An upper limit on the production cross section was set. As a side product exclusive high mass dilepton production $\left(40<M_{l l}<75 \mathrm{GeV} / \mathrm{c}^{2}\right)$ was measured that is in good agreement with theoretical predictions.

\subsection{Early Exclusive Photon Pair Production}

An earlier search of exclusive $\gamma \gamma$ production in hadron-hadron collisions already found evidence $[\mathbf{1 1}]$, but with only three candidate events. These candidates with 
$E_{T}(\gamma)>5 \mathrm{GeV}$ and $|\eta|<1.0$ have been extracted from data of $532 \mathrm{pb}^{-1}$ integrated luminosity. Two of the three events had a single narrow electromagnetic shower on each side as expected for $\gamma \gamma$ events. The theoretical prediction was $0.8_{-0.5}^{+1.6}$ events [1]. An observation could not be claimed. 


\section{CHAPTER 5}

\section{Analysis Methodologies}

\section{General Description of Analysis}

Particle physics at high energy colliders can be studied in many different ways using various methods and tools. The results are usually statistically formatted to be compared and discussed with theoretical predictions. This chapter will briefly define the type of measurements used here and list all the tools needed to obtain the final results.

\subsection{Cross Section Evaluation}

Our main interest has been the observation of the CEP of diphotons and the measurement of the cross section under certain conditions. An observation is given in case a certain number of signal events are observed and it is proven that those are not due to background fluctuations. The statistical significance (p-value) for an observation has to be greater than $5 \sigma$ (standard deviations) compared to a normal Gaussian distribution centered at 0 . Details will be discussed later. To obtain the cross section the measurement can be stripped down to a simple counting experiment. Most importantly one needs to count the visible signal candidate events and estimate the possible background events. Together with the integrated luminosity $\mathcal{L}_{\text {int }}$ and measurements of the efficiencies of the counting procedures one can calculate the cross section with the simple formula,

$$
\sigma=\frac{\left.N(\text { candidates })-\sum_{i} N_{i} \text { (background }\right)}{\mathcal{L}_{\mathrm{int}} \prod_{j} \varepsilon_{j}},
$$

where $\varepsilon_{j}$ stands for efficiency/acceptance corrections for each involved selection procedure, e.g. trigger efficiency, reconstruction efficiency, photon identification efficiency, the efficiency of being able to select exclusive events, etc. For $\sigma$ read $\sigma\left(E_{T}(\min ),|\eta(\max )|\right)$. The signal candidates are obtained via a selection chain starting from the online trigger selection. Subsequently events are selected from the recorded datasets that pass certain selection cuts. The efficiency for every applied operation needs to be estimated. Finally, possible background processes contaminating the signal events have to be estimated. In the following the outline of the whole measurement of 
the CEP of $\gamma \gamma$ is explained. In parallel to the diphoton study, the CEP of electron positron pairs is also measured. This process has a well known cross section via QED mediated photon fusion, $\gamma \gamma \rightarrow e^{+} e^{-}$, and it was already earlier successfully measured at CDF consistent with theoretical predictions. Both the $e^{+} e^{-}$and the $\gamma \gamma$ measurement can use the same analysis procedures, as photons and electrons have similar signatures in the detector except that electrons leave tracks in the tracking devices. By re-measuring successfully the CEP of $e^{+} e^{-}$, sufficient confidence is gained to also measure the CEP of $\gamma \gamma$. Therefore simultaneously both class of events are selected. The separation of events into those with two charged particle tracks and those with no tracks is left until the final stage, with any events that are not distinctly in either class studied as possible backgrounds. These can be exclusive $\gamma \gamma$ events with a photon conversion, or exclusive $e^{+} e^{-}$events with a Bremsstrahlung or $\delta$-ray.

1.1.1. Trigger and Event Selection. In the CDF experiment no proton or antiproton taggers have been installed that could select CEP events with great signal to background ratio. Thus, the surviving protons and antiprotons are unseen. Therefore one has to rely entirely on methods selecting events with large rapidity gaps on both sides of the centrally produced system. The first step for the CEP event selection is already included in the trigger, which will be discussed in detail in Chapter 6 Section 1.1. The complex hardware and higher level trigger system of the CDF experiment was already introduced in detail in Section 2.6 of Chapter 3. The trigger is based (at level 1) on one EM shower in the calorimetry with an $E_{T}>2 \mathrm{GeV}$ plus a veto on hits (void of particles) in the BSC-1 devices placed in the forward region. This limited rapidity gap requirement from $5.4<|\eta|<5.9$ already reduces the majority of inelastic background events. At higher trigger level two EM showers with $E_{T}>2 \mathrm{GeV}$ are required. Note that the trigger accepts exclusive $e^{+} e^{-}$events as well as exclusive $\gamma \gamma$ events.

The next step in the selection chain is the offline selection of events that include the main signature of exactly two EM object in the calorimeter, which will be discussed in detail in Chapter 6 Section 1.2. The third step selects all events that show exclusive signatures. This exclusive filter requires no particles in all the various detector components except the two EM showers, that constitute the signal. The outgoing protons and antiprotons will be far too forward to be detected as already mentioned above; they escape down the beam pipes. However if they dissociate, e.g. $p \rightarrow p \pi^{+} \pi^{-}$, one or more of the dissociation products is likely to give rise to hits in the BSC stations, and the event will be rejected. More details will be discussed in Chapter 6 Section 1.3. 
The final steps are to apply quality cuts to select the cleanest candidates and to separate those from backgrounds, which will be discussed in detail in Chapter 6 Section 1.4. To minimize backgrounds it was decided to restrict the minimum transverse energy of the EM objects to $E_{T}>2.5 \mathrm{GeV}$ and the fiducial region to $|\eta|<1.0$. Although this removes about half of the signal candidates, low background is favored over better statistics.

1.1.2. Efficiency Studies. Selection methods in high energy physics are never $100 \%$ efficient. Using traditional cutting techniques good signal candidates could be missed if cuts are too conservatively tight. Unwanted background events could be selected by using too relaxed cuts. It is essential to measure the efficiency of each applied operation in the selection process.

The trigger efficiency is measured using data that was recorded by a trigger not correlated with the signal trigger. This will not bias our results. Details can be found in Chapter 6 Section 1.1.

Next, the efficiency of recording and reconstructing the signal events needs to be estimated (see Chapter 6 Section 1.2). Due to non-optimal geometrical and kinematical coverage of the CDF Run II detector, this is not only an offline reconstruction question. Moreover, the efficiency of the physical detector and of the offline reconstruction is convoluted. Offline reconstruction efficiency means: How well are the objects of interest detected and determined correctly from raw low-level detector data? The detector and reconstruction efficiency used here rely on detailed Monte Carlo simulations.

The efficiency for detecting an exclusive events is measured by randomly recorded data that is selected by bunch crossing times and not biased by any trigger selection (see Chapter 6 Section 1.3). The CDF standard procedure is applied here that was used successfully by several earlier analyses (see the previous Chapter 4).

Additional photon identification efficiencies are estimated as well, which will be discussed in detail in Chapter 6 Section 1.4.

1.1.3. Backgrounds. The discussion of possible background processes faking a signal event is of great importance. One possible background is exclusive $\mathbb{P}+\mathbb{P} \rightarrow \pi^{0} \pi^{0}$. With the help of the CES strip/wire chambers (see Chapter 3 Section 2.3.1) it is shown that this background is very small (consistent with zero). A photon leaves a clean signal, in the ideal case with a single cluster in the proportional chambers at the shower maximum position as shown in Figure 5.1. In case of a $\pi^{0}$ that decays to two photons with a small opening angle, one expects more than one cluster in those devices and can exclude such events. CES modules are embedded in all central calorimeter 


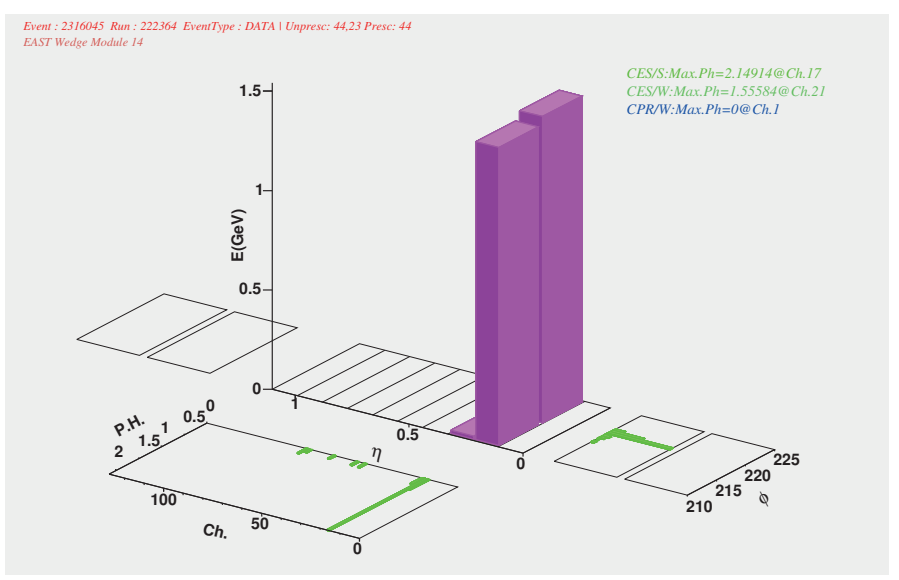

FiguRE 5.1. The signal of a photon in the CES strip/wire chamber embedded in a central calorimeter tower.

towers. Cases in which the two photons are spread over different towers are canceled by the strict exclusive filter mentioned above.

Other possible backgrounds to be discussed are CEP events with dissociating outgoing (anti)protons, cosmic rays and non-exclusive background. Details of the background estimation are discussed in Chapter 6 Section 4 .

\subsection{Statistical Methods}

It is common in experimental high energy physics to express the significance of a result in terms of $p$-values, i.e. how well is an observed signal distinguished from background processes. Often the $p$-value is the outcome of a hypothesis testing procedure from a frequentist point of view. In case one has two hypotheses, $H_{0}$ a tested and trusted and $H_{1}$ a new and alternative hypothesis, one would like to obtain the probability of a measurement of $\xi$ being correctly described by one of the hypotheses. Here $\xi$ is called the test statistic. The parameter space of $\xi$ is divided into areas where one of the hypothesis is more likely to be true. One can calculate the significance level in case $H_{0}$ is true and $\xi$ is outside the favorite region:

$$
\alpha=\int_{\xi_{\text {cut }}}^{\infty} g_{0}(\xi) d \xi,
$$

where $g_{0}(\xi)$ is the probability density function for $\xi$ in case of $H_{0}$. Suppose hypothesis $H_{0}$ is true and rejected, one talks about an error of Type 1 . A Type 2 error would be 
the incorrect rejection of the true hypothesis $H_{1}$ with a probability of,

$$
\beta=\int_{-\infty}^{\xi_{\mathrm{cut}}} g_{1}(\xi) d \xi
$$

One calls the probability $1-\beta$ the power of the statistical test.

In the case of a counting experiment as presented in this work one is interested in the significance of the result describing new physics. Given the number of observed candidate events $n_{s}$ one can test if they are consistent with just being the result of a random background fluctuation which is the null hypothesis $H_{0}$. The $p$-value for such a test can be interpreted as the probability of observing at least as many candidates as in the test, given that the null hypothesis $H_{0}$ is true. If the expected background rate $n_{b}$ is estimated and both signal and background are expected to be randomly Poisson distributed, the $p$-value can be computed by,

$$
p=\sum_{n \geq n_{s}} \frac{e^{-n_{b}} n_{b}{ }^{n}}{n !} .
$$

Often such a $p$-value is interpreted in terms of Gaussian statistics, i.e. the probability that a process is observed a certain number of standard deviations $(\sigma)$ off the mean of a standard normal centered around zero.

In reality this simple procedure is often not applicable as there are statistical and systematic uncertainties in the background rates. In this case one needs to look for alternative ways to estimate the significance of the result. The method used here is the prior predictive method [128]. It employs a Bayesian prediction of the probability density of the data and the background. The distribution is expressed as,

$$
p(x \mid A)=\int p(x \mid \theta) p(\theta \mid A) d \theta,
$$

where $p(\theta \mid A)$ is the parameter distribution $\theta$ under some assumption $A$ before the measurement. As a result, one has the distribution of the observable data observed under correct assumptions. For Poisson distributed data with the assumption of Gaussian errors the prior predictive $p$-value can be calculated by [129],

$$
p_{\text {prior }}\left(n_{0}\right)=\int_{0}^{\infty} \frac{\exp \left\{-\frac{1}{2}\left(\frac{\nu-x_{0}}{\Delta \nu}\right)^{2}\right\}}{\sqrt{2 \pi} \Delta \nu \frac{1}{2}\left[1+\operatorname{erf}\left(\frac{x_{0}}{\sqrt{2} \Delta \nu}\right)\right]}\left(\sum_{n=n_{0}}^{\infty} \frac{e^{-\nu} \nu^{n}}{n !}\right) d \nu,
$$

where $n_{0}$ is the number of observed events, $\nu$ the number of expected background events (null hypothesis), $x_{0}$ the mean (background) of the truncated Gaussian prior and $\Delta \nu$ the uncertainty on the mean (background). 


\subsection{Data, Events and Variables}

An event is an entity defined as the recordings of physical occurrences in a collision of two bunches at the interaction point inside the detector. All possible information obtained by the various subdetectors is collected and saved inside the event (see Chapter 3 Section 2.6.3). In addition, reconstructed high level objects such as tracks, identified particle objects (electrons, muons, jets, etc) are added to the event offline.

Some frequently used variables that define objects in the events are:

- $E_{T}$ : The transverse energy is the projection of the energy $E$ into the azimuthal plane ( $x$-y-plane, see Figure 3.7). Using the polar angle $\theta$ one gets $E_{T}=E \sin \theta$. The energy $E$ is the sum of the electromagnetic and hadronic cluster energies deposited in the calorimetry. Corrections are applied to the raw energy deposits.

- $p_{T}$ : The transverse momentum is defined as the momentum projection into the azimuthal plane. The momentum $p$ associated with particles leaving tracks in the tracking devices is obtained by fitting the curvature of the track.

- Azimuthal angle $\phi$ and pseudorapidity $\eta$ : The azimuthal angle $\phi$ is an angle measurement in the $x$ - $y$-plane with being zero along the $x$-axis (see Figure 3.7). The pseudorapidity $\eta$ is directly related to the polar angle $\theta$ for approximately massless particles at high energy collider experiments (see Appendix 2, Equation (126)).

- Separation angle $|\pi-\Delta \phi|$ : This variable shows the azimuthal opening angle, a useful measure for the back-to-backness of the products of a two body process. Here, the subtraction of the angle from $\pi$ is found to be useful when dealing with binned data.

- 3D opening angle (rad): This variable is the opening angle between two off-flying particles in the 3-dimensional Euclidean space. A useful quantity to filter out cosmic ray background with an 3D opening angle close to $\pi$.

- $E_{\mathrm{HAD}} / E_{\mathrm{EM}}$ : The ratio between the EM and hadronic energy deposits of a cluster is an important variable to select electron and photon candidates. For electrons and photons the ratio is expected to be small. An energy depending correction factor is added to scale the ratio cut with energy.

- Invariant mass $M_{\text {inv }}$ : The invariant mass can be defined for a system of certain energy and momentum invariant of the reference frame. In the center of mass frame the invariant mass is simply the mass itself. For a massless two-body system the invariant mass is calculated by

$$
M_{\mathrm{inv}}^{2}=\left(p_{1}+p_{2}\right)^{2}=2\left|\boldsymbol{p}_{1}\right|\left|\boldsymbol{p}_{2}\right|(1-\cos \Delta \phi) .
$$




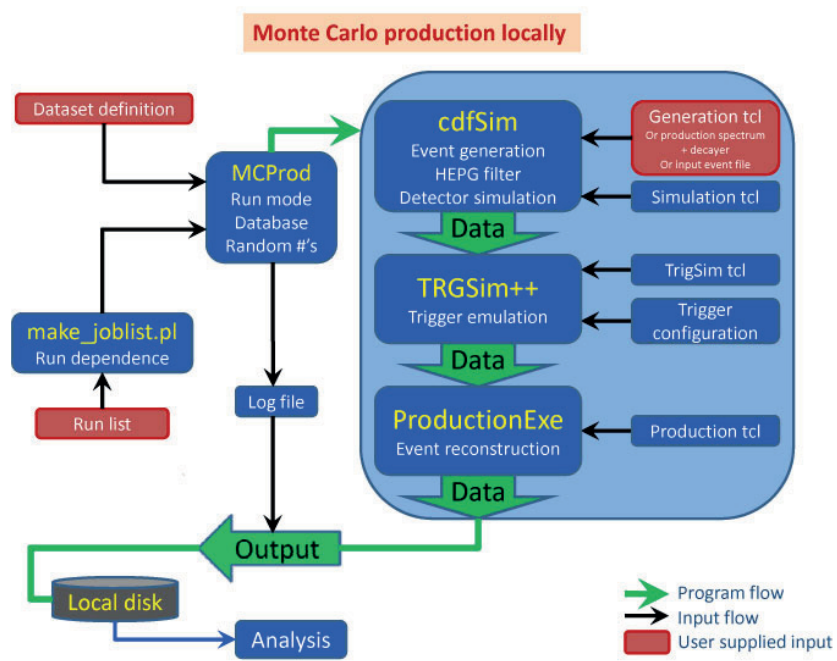

Figure 5.2. Chart of the CDF Monte Carlo production chain. Plot taken from the CDF collaboration.

- $p / E$ : A useful measure for the quality of electron tracks. Electrons loose energy via Bremstrahlung passing through material in an EM field that results in a lower $p_{T}$. An ideal tracking detector without material yields $p / E=1$.

\section{Physics Simulation}

In this analysis simulated data is used to some extent. Despite the wish to have an entirely data-driven analysis, using simulated data is very often the only way to obtain reliable results. In this analysis simulated data is used to estimate some of the measurement efficiencies and acceptances and to compare the kinematics of the final results. The data is simulated by Monte Carlo methods using all present knowledge about the physics involved at the time of the experiment. The experimental apparatus is modeled with details close to reality. A vast number of adjustable parameters allow the tuning of the simulated data to represent presently known physics as best as possible on the way of searching for new unkown physics. A factor that has to be taken into account is the computation time that is a real constraint in terms of simulated details. A typical simulation chain starts with a physics Monte Carlo generator that generates particle collisions. This is followed by the Monte Carlo physics simulation of the experimental apparatus that uses the generated physics process as input and simulates the particles traversing the detector. The next step is the digitization to simulate the detector response similar to the output of the data-acquisition system 
that processes the information of real recorded collisions. The reconstruction and analysis tools can be commonly used both on real and simulated data. In Figure 5.2 a chart of the complete Monte Carlo production complex used at CDF is shown. Next, the tools are described in more detail that are used in this analysis.

\subsection{Events Generators}

Event generators are software tools that simulate high energy particle collisions using computer generated random numbers. Usually, an event generator calculates cross sections of physics processes as well as a full kinematical description of the outgoing products. One separates between general purpose event generators such as PYTHIA [114] or SHERPA [115] and special purpose generators as for example SUPERCHIC. Before switching to the specialized ones, some common features are listed that most general purpose generators include:

- Hard scattering process simulation at leading order (next to leading order or possible higher orders in some generators) including decay channels.

- Initial and final state radiations.

- Utilizing of factorization as much as possible.

- Simulation of underlying events.

- Fragmentation and hadronization.

- Energy and momentum conservation.

- Mostly standardized particle ID codes and data output format.

All event generators simulating hadron-hadron collisions use heavily parton distribution functions as the colliding particles have complicated composite structures. At CDF all Monte Carlo generators use by default CTQE5L parton distribution functions provided by the CTEQ collaboration [116].

2.1.1. SuperCHIC, ExHuME. Both the SuperCHIC $[\mathbf{1 1 7}, \mathbf{1 1 8}]$ and the ExHUME [107] programs simulate only CEP processes for various collision energies based on the KMR model that is described to some detail in Chapter 2 Section 2.4. Those are presently the only generators that simulate the CEP of diphotons. ExHUME was primarily written for the CEP of the SM Higgs boson and dijets that are not provided so far by the SUPERCHIC program. However the latter is clearly advanced in the light meson sector covering presently the CEP of $J / \Psi, \Upsilon, \chi_{c}, \chi_{b}, \eta_{c}, \eta_{b}$ as well as meson pairs $\pi \pi, \eta \eta, \eta^{\prime} \eta, \eta^{\prime} \eta^{\prime}, \rho \rho$. Some spin configurations and decay channels of the mesons are included. ExHuME that was developed prior to SUPERCHIC has some limitations regarding the implementation of the KMR model especially in the low mass sector. It is supposed to be used only for higher mass systems, which is the main reason why 
SUPERCHIC was chosen to simulate exclusive diphoton events for this analysis. The authors of ExHuME chose to use a constant value for the survival factor $S^{2}$ per collision energy. SUPERCHIC on the other hand has properly implemented the survival factor depending on the type of CEP process, the impact parameter and rapidity. In addition the enhanced survival factor is taken into account. The latest version also includes full kinematics of the outgoing hadrons opening the possibility to interface a third party application such as PYTHIA or the Minimum-Bias Rockefeller Monte Carlo MBR for the fragmentation of possibly excited hadrons. SUPERCHIC users can choose between the MRST99 [119] or the MSTW08LO [26] parton distribution functions in the version 1.3 used in this analysis. However, it is not difficult to add others into the code.

2.1.2. LPair. The LPAIR Monte Carlo generator $[\mathbf{1 2 0}]$ is specialized in producing CEP via two-photon interactions as outlined in Chapter 2 Section 3. Details to the exact theoretical model implemented can be found in reference [121]. Due to limitations of the EPA [79] approach at low t-channel momentum transfer it features a full matrix element calculation. The generator delivers full kinematical information of all product particles including the outgoing (anti)protons. However, for the inelastic cases with excited (anti)protons, fragmentation models are not implemented. Instead third party applications such as PYTHIA or the MBR generator have to be interfaced to dissociate the outgoing hadrons. One has to be careful in interpreting the results of this generator as soft rescattering effects of the colliding hadrons are not included. This generator is used to simulate the CEP of $e^{+} e^{-}$pairs acting as a control channel for the search for exclusive $\gamma \gamma$ production.

2.1.3. MBR. This generator was originally written for the usage at the CDF experiment. The simulation covers diffractive physics, i.e. single- and double-diffraction as well CEP. Additionally it predicts the total, elastic and inelastic cross section over the accessible energy scale tuned and normalized to the CDF measurements. The simulation model is a parametrization based entirely on the Regge formalism that we discussed in detail in Chapter 2 Section 2.2.2. Presently the MBR has been included into the latest PYTHIA 8 Monte Carlo generator [122]. In this study a subroutine of the MBR was used that fragments excited hadron states. It was interfaced with the LPAIR program to estimate the amount of unseen inelastic CEP processes as background to the elastic ones. 


\subsection{CDF Detector Simulation}

The complexity of CDF detector simulation framework [123] (version 6.1.4) is best seen in Figure 5.2. The three main parts consist of the CDFsIM package simulating the detector physics and response, the TRGSIM ++ package offering a trigger simulation and the PRODUCTIONExE package performing the event reconstruction.

The CDFSIM processes the output of the event generators. Major event generators can be run from within the Monte Carlo Production framework. Some specialized external generators, e.g. SUPERCHIC, can feed standalone produced event-files via the standardized STDHEP format $[\mathbf{1 2 4}]$ into the framework. The CDF detector is modeled within and simulated with the GEANT3 package [125]. The sensitive material as well as large part of the inactive passive material is added in great detail. The physical interaction with the material is mostly done by GEANT3. However some parts are using different techniques to save computing time as a full physics simulation can be quite time consuming especially when, for example, simulating a large amount of high $p_{T}$ events in the order of millions.

To optimize the time consumption, the calorimeter simulation, for example, uses GFLASH [126] a fast simulation toolkit, based on parametrized longitudinal and lateral profile models of EM and hadronic shower development inside materials. GFLASH uses information obtained from GEANT3 that simulates tracking and inelastic collisions. The energy distribution and deposition in the sensitive volumes that would consume a large amount of cpu-time is done entirely by GFLASH. The simulation is well tuned by electron and pion testbeam data for the EM and hadronic calorimeters, respectively, over a large energy range from $<1 \mathrm{GeV}$ to $>100 \mathrm{GeV}$.

The GARFIELD [127] package provides the drift models used to simulate the COT, the gaseous wire drift chamber tracking detector. Accompanying tools were used to simulate energy losses of moving particles in gases and gas transportation properties. The developed parametrization model agrees well with test data in terms of tracking properties and particle separation powers.

Also the silicon vertex detector uses a parametrization that takes into account the mean free path length of the ionizing particles to estimate the charge deposition on each strip with the help of Landau fluctuations. The silicon wafer sectors holding the strip structure are chosen by track extrapolation. Possible track deviations due to multiple scattering are considered as well.

Some for this study relevant detectors, the forward BSCs and the forward Miniplug, are not simulated within the CDF simulation framework. However, fortunately none of the results within this work depend on the simulation of those detectors. 
The event generation, the detector and trigger simulation are comparable to taking real data. The following production or event reconstruction concerns both simulated and real data and is identical for both.

\section{Analysis Tools and Computing Framework}

The reconstructed data, real and simulated, is not yet user friendly. An additional layer of data structure for the end-user analysis is needed. The process is called Ntupling and will run over the fully reconstructed data to extract and format information according to the interest of the data analyzing groups. The ntupled data is stored in a ROOT-based binary format. The data is optimized for size (large reduction compared to reconstructed data) and usability and to some extent includes corrections to the reconstructed data. Commonly used ntuple types are the STNtuple, BSTNtuple and TopNtuple. For this work the STNtuple data format is used and described. The structure of such a ntuple is a ROOT tree with branches holding the different objects. Leaves inside the branches contain variables of those objects. An object, as for example the TStnPhoton, contains variables such as the energy $E$, the position information $\eta$ and $\phi$, the ratio of hadronic to electromagnetic energy, etc. Ntuples can be fully analyzed with ROOT-based scripts.

A full object oriented framework for the STNtuple formatted data has been built. Each reconstructed object has its own class with methods to access relevant variables. A basic analysis code consists of two classes. The TStnAna class forms an object that loops over all events within the STNtuple. The class TStnModule forms the object that is linked to the TStnAna and executed within the event loop. A module for example could be a filter for events that fulfill a certain trigger path.

For this work customized modules have been written on top of the STNtuple framework to retrieve, select and characterize the data used here. 



\section{CHAPTER 6 \\ Search for Exclusive Photon-Pair Production}

\section{Selection of Exclusive Electromagnetic Showers}

For this analysis CDF Run II data has been used. The data was taken from June 2006 to August 2007 (Periods 8 to 13, Runs 219887 to 246229). We used an official Good Run list for electron based analyses from the Data Quality Monitoring group, but removed data taking runs that had been marked bad for the Miniplug, the CES or PES shower max detectors as well as for the BSC stations. We did not require good data from the silicon tracking detectors. We found that the Miniplug detector behaved differently in its response for high and low instantaneous luminosities in some runs in Period 12 compared to other periods, and removed those runs as well.

The data is available as production level ntuples (gdif0i/j) and in STntuple format processed via gen6 production framework (gdifai/j).

\subsection{The DIFF_DIPHOTON2 Trigger}

The DIFF_DIPHOTON2 trigger, a L3 trigger within PHYSICS_4_* table especially developed for central exclusive physics involving low $E_{T}$ photons and electrons, was used. It followed the DIFF_DIPHOTON trigger that was designed and installed in 2004. Key points of this trigger are the $2 \mathrm{GeV}$ threshold for electromagnetic (EM) objects

TABLE 6.1. Key details of the DIFF_DIPHOTON2 trigger from physics table PHYSICS_4_*.

DIFF_DIPHOTON2 trigger information

Level 1: Veto on East and West BSC1 counters, One $2 \mathrm{GeV} \mathrm{E}_{\mathrm{T}} \mathrm{EM}$ object in the central or plug calorimeter, $\mathrm{HAD} / \mathrm{EM}$ ratio of 0.125 in central and 0.0625 in plug region.

Level 2: 2 EM objects in central or plug calorimeters with $|\eta|<2.61, E_{T, \min }$ of $2 \mathrm{GeV}$ and $\mathrm{HAD} / \mathrm{EM}$ ratio of $<0.125$.

Level 3: 2 EM objects with $E_{T, \min }$ of $2 \mathrm{GeV}$, central CES $\chi^{2}$ cut of 20.0, ISO $<4 \mathrm{GeV}$ and ISO-ratio $<0.10$ for central and plug regions. 


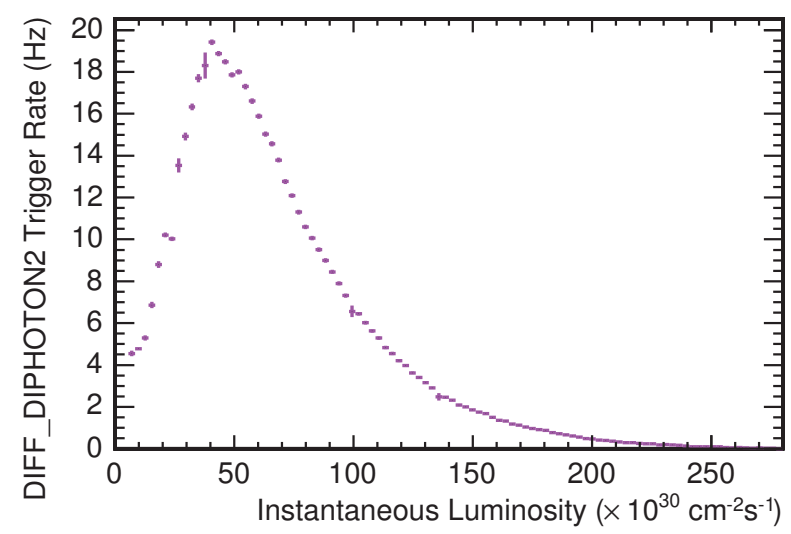

FiguRE 6.1. DIFF_DIPHOTON2 trigger rate versus instantaneous luminosity. Run selection requirements: PHYSICS_4_\% trigger table, minimal luminosity of $10 \mathrm{nb}^{-1}$ and run type PHYSICS between run 219887 and 246231.

in the central and plug calorimeters, and a veto on particles in the BSC- 1 stations on both sides of the interaction point. Previously in version 1 of the trigger the $E_{T}$ threshold for EM objects was at $4 \mathrm{GeV}$. The rapidity gap requirement in the trigger using the BSC-1 stations makes it possible to lower the $E_{T}$ threshold down to such a low energy as $2 \mathrm{GeV}$ by rejecting a large fraction of inelastic collisions as well as most events with more than one interaction (pileup). More details on the trigger can be found in Table 6.1. Using this trigger and the final Good Run list, we have data from $1.114 \mathrm{fb}^{-1}$ integrated luminosity. The offline correction factor of 1.019 is included to compensate for the difference between online and true luminosities.

The DIFF_DIPHOTON2 trigger rate as shown in Figure 6.1, peaks at an instant luminosity of around $40 \mu \mathrm{b}^{-1}$ per second $\left(40 \times 10^{30} \mathrm{~cm}^{-2} \mathrm{~s}^{-1}\right.$ for shorthand) with a rate close to $20 \mathrm{~Hz}$. The decrease above this value is due to multiple interactions per single bunch crossing. In such circumstances rapidity gaps are more likely to be destroyed, hence the trigger rejects such events. In version 1 of the trigger we had a trigger rate of $0.11 \mathrm{~Hz}$ at about $20 \mu \mathrm{b}^{-1} \mathrm{~s}^{-1}\left(20 \times 10^{30} \mathrm{~cm}^{-2} \mathrm{~s}^{-1}\right)$. Due to the strong and efficient rapidity gap requirement both triggers could run without pre-scaling.

For all available data that included the DIFF_DIPHOTON2 trigger we obtained about 200 million triggered events.

Trigger Efficiency. To obtain the efficiency for triggering on exclusive events of interest we used photons from the Minimum-bias dataset that passed all photon 
TABlE 6.2. Photon ID cuts for trigger efficiency study.

\begin{tabular}{ll}
\hline & Photon ID cuts \\
\hline$E_{T}$ & $>1 \mathrm{GeV}$ (for trigger eff. study) \\
CES $\chi^{2}$ & $<20.0$ (central), 10.0 (plug) \\
HAD/EM ratio & $<0.056$ (central) 0.005 (plug) \\
CES geometry & $|x|<21.0 \mathrm{~cm} \& 9.0<|z|<230 \mathrm{~cm}$. \\
\hline
\end{tabular}

identification cuts. Using those photons we were not biased by any particular trigger, as the Minimum-bias data is triggered via coincident hits in both the east and west CLC. We selected events with exactly one photon that passed the photon identification cuts recommended by the CDF photon group. The cuts used are shown in Table 6.2. The CES $\chi^{2}$ cut is based on the $\chi^{2}$ value of the lateral shower shape compared to the expected one for photons. The CES geometry cut is based on the fiducial area of the CES strip/wire chambers. These probe photons were put through a trigger filter to find out if they would be accepted by the DIFF_DIPHOTON2 trigger. The efficiency is,

$$
\varepsilon_{\text {trig }}=\frac{\# \text { Photons }_{\text {probe }}^{\text {trig }}}{\# \text { Photons }_{\text {probe }}}
$$

with the probe photons that pass the trigger filter in the numerator. To find out if a photon would be accepted we used the recorded offline trigger information for Level 2 and 3. Every photon that passes the Level 2 trigger has all the requirements to pass Level 1. Therefore it is sufficient just to study Level 2 and Level 3. All applied Level 2 and Level 3 cuts can be found in Table 6.3. Trigger and offline photons were matched via their calorimeter seed tower. The results of the trigger efficiency study can be

TABLE 6.3. Level 2 and Level 3 trigger simulation cuts for the efficiency study.

\begin{tabular}{llll}
\hline \multicolumn{4}{c}{ Trigger simulation cuts } \\
Level 2 & & \multicolumn{2}{c}{ Level 3} \\
\hline L2_Et & $\geq 2.0 \mathrm{GeV}$ & L3_Et & $\geq 2.0 \mathrm{GeV}$ \\
L2_HadEm & $\leq 0.125$ & L3_HadEm & $\leq 0.125$ \\
L2_ABS_ETA_MAX & $\geq 2.61$ & L3_CesAvgChiSq & $\leq 20.0 \mathrm{GeV}$ \\
& & L3_TotIso4 & $\leq 2.0$ \\
& & L3_RatioTotIso4 & $\leq 2.0$ \\
\hline
\end{tabular}

seen in Figure 6.2 and 6.3. In Figure 6.2 the efficiency is shown as a function of the photons transverse energy $E_{T}$, the pseudorapidity $\eta$ and the azimuthal angle $\phi$. The $E_{T}$ cut of $2.5 \mathrm{GeV}$ used for the final trigger efficiency numbers is shown as red line in the efficiency plot as a function of $E_{T}$. In Figure 6.3 on the left the $E_{T}$ distribution 

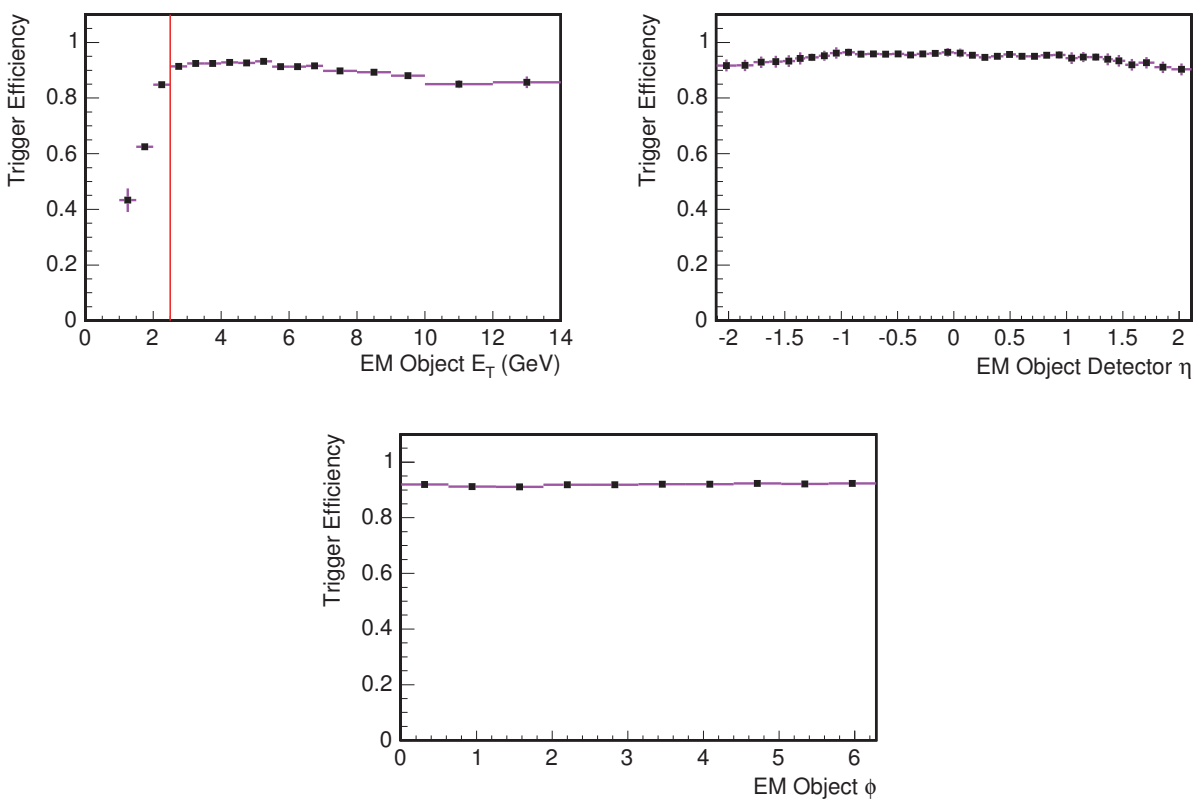

Figure 6.2. Trigger efficiency plots as a function of $E_{T}, \eta$ and $\phi$ using the final $E_{T}$ cut of $2.5 \mathrm{GeV}$.
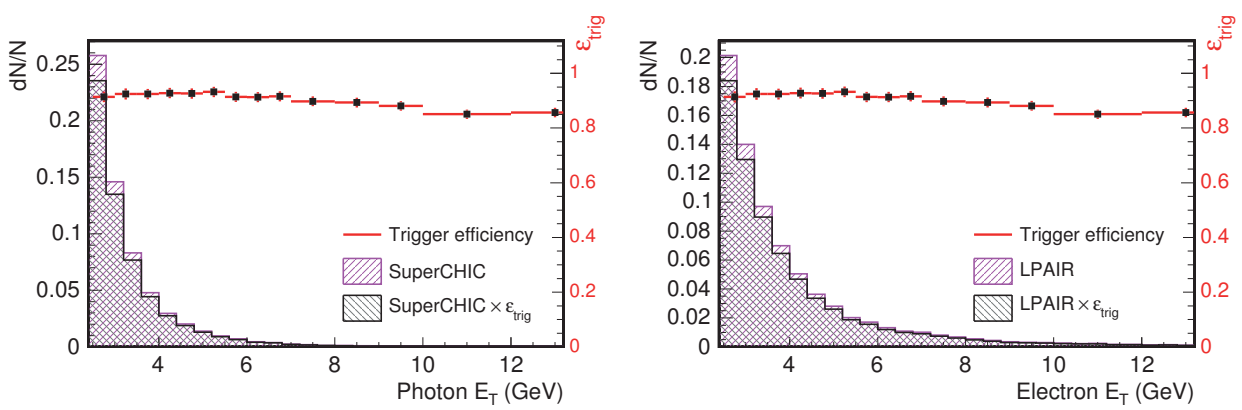

Figure 6.3. Trigger efficiency plot as a function of $E_{T}$. The black histogram represents the convolution of the signal photon $E_{T}$ distribution with the trigger efficiency. The total trigger efficiency was obtained by dividing the black histogram with the pink histogram. The final $E_{T}$ cut of $2.5 \mathrm{GeV}$ was used. 
of the exclusive Monte Carlo photon sample is shown as well as its convolution with the trigger efficiency over $E_{T}$. The total trigger efficiency is obtained by dividing the convoluted histogram (black) with the ideal histogram (pink). Weighting is necessary as the minimum bias photons show a different slope in the $E_{T}$ distribution. It is sufficient to convolute only the $E_{T}$ distribution as the distributions over $\phi$ and $\eta$ do not show any difference between minimum-bias data and our signal. Due to different $E_{T}$ distribution of the exclusive electrons we needed to estimate the trigger efficiency for our "control channel" separately. The measured trigger efficiencies are listed in Table 6.4. The systematic error was estimated by varying the efficiency bin-wise

TABLE 6.4. DIFF_DIPHOTON2 trigger efficiency. The trigger efficiency estimate is averaged over the kinematic range of $\left(E_{T}>2.5 \mathrm{GeV},|\eta|<1.1\right)$.

\begin{tabular}{lccc}
\hline & $\varepsilon_{\text {trig }}$ & Stat Err & Syst Err \\
\hline Photons & 0.920 & \pm 0.005 & \pm 0.018 \\
Electrons & 0.918 & \pm 0.009 & \pm 0.018 \\
\hline
\end{tabular}

by $\pm 2 \%$ in the weighting process using the $E_{T}$ distribution of the Monte Carlo sample, which was motivated by the energy-scale uncertainty measured by CDF [130]. The rapidity gap requirement in form of the BSC-1 veto within the Level 1 trigger was taken to be $100 \%$ efficient. In the case of any inefficiency our overall trigger efficiency would have been overestimated. However, the trigger requirement on the BSC-1 counters had been $<1000$ ADC counts and our offline requirement $<400$ ADC counts. The trigger threshold was clearly above the noise level and the offline selection criteria. Therefore we expected close to $100 \%$ of our offline events to pass the trigger. This was already earlier studied, e.g. for the exclusive Charmonium study [82] with similar cuts (trigger and on-line). During data taking the BSC-1 devices had not been very noisy nor inefficient. Data taking runs with malfunctioning BSCs that could have affected the trigger efficiency had been removed.

\subsection{Preselection of EM Events}

After applying the trigger filter and the Good Run selection we preselected events that had exactly two EM objects in the central and plug calorimeters. The central and mid-plug calorimeters include all towers from 0 to 17 with an $|\eta|$ coverage of $0.0<|\eta|<2.11$ (see Table 6.7 for detailed information about the calorimeter segmentation). These EM objects could be anything that deposits mostly electromagnetic energy and are reconstructed as a general STntuple photons. This reconstruction 
object class contains also all electron type objects. At this stage we were left with approximately 94 million events with exactly two EM objects.

Reconstruction Efficiency. The reconstruction efficiency for electromagnetic objects can be obtained by using photon pair events generated by the SUPERCHIC Monte Carlo computer program. The Monte Carlo events were processed by the CDF simulation and reconstruction software. This addresses the efficiency of the CDF detector as well as the efficiency to reconstruct basic electromagnetic objects at the calorimeter level. Knowing the number of generated EM objects we counted the number of events that survived the detector simulation and were successfully reconstructed. The efficiency was obtained by dividing the number of filtered events by the total number of generated events:

$$
\varepsilon_{\mathrm{rec}}=\frac{\# \text { Events }_{\mathrm{rec}}}{\# \text { Events }_{\mathrm{gen}}}
$$

The level of reconstruction is the loosest available quality level of photons that are categorized as TStnPhotons. At this level also electrons are within this class of reconstructed objects. Therefore we used the same method to obtain reconstruction efficiency for the electron-pair study that acts as a control channel. However, due to the different slope of the exclusive electron-pair cross section compared to the exclusive photon-pair production we used an exclusive electron-positron pair Monte Carlo sample produced by the LPAIR Monte Carlo program for the electron study. Due to possible correlations between the two outgoing objects, the efficiency refers to the whole event and not a single EM object. As we were only interested in the central EM objects we cut for $|\eta|<1$. In Figures 6.4 we can see the detector and reconstruction efficiency product versus the transverse energy $E_{\mathrm{T}}$, the azimuthal angle $\phi$, and the pseudorapidity $\eta$ of the EM objects. These plots represent the result for the final used $E_{\mathrm{T}}$ cut of $2.5 \mathrm{GeV}$ shown as red line in the above Figure. The systematic error was estimated by varying the input energy scale by $\pm 2 \%$. The difference in the efficiency result was taken as a systematic error. The value of $2 \%$ is justified by the energy scale studies at CDF [130]. They estimated a systematic uncertainty of $1 \%$ on the electromagnetic energy scale using the difference between data and Monte Carlo simulation down to an electron momentum of $2 \mathrm{GeV}$. Being conservative we doubled that uncertainty and use it for both the electron and photon reconstruction efficiency estimate. In Table 6.5 we can see the results for different $E_{T}$ cuts and $|\eta|<1.1$ of photon-pairs at reconstruction level. The reconstruction efficiencies for central electron pairs for different $E_{T}$ cuts can be found in Table 6.6. 

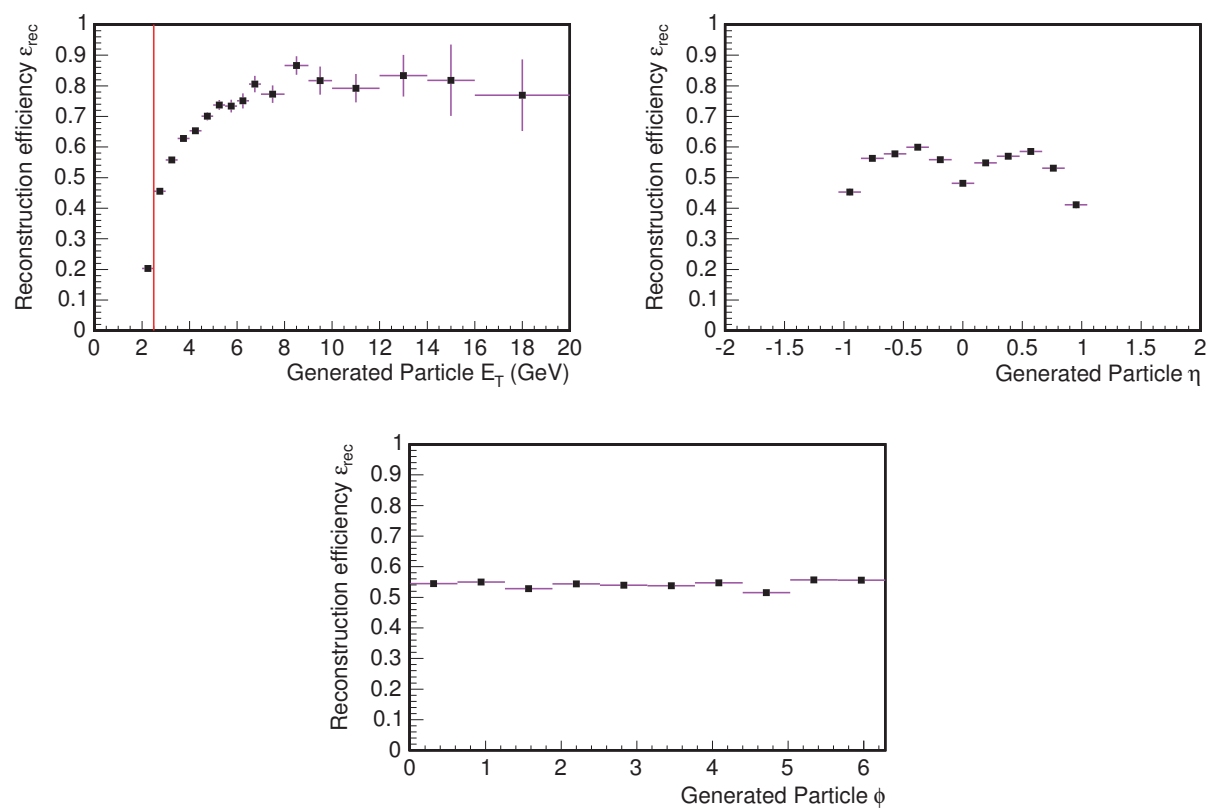

Figure 6.4. Reconstruction efficiency as a function of $E_{T}, \eta$ and $\phi$. The red line represents the final used $E_{T}$ cut of $2.5 \mathrm{GeV}$.

TABLE 6.5. TStnPhoton reconstruction efficiency averaged for different $E_{T}$ cuts and $|\eta|<1.1$. The full CDF simulation and reconstruction software was used.

\begin{tabular}{lccc}
\hline$E_{\mathrm{T}}$ cut $(\mathrm{GeV})$ & 2.0 & 2.5 & 5.0 \\
\hline$\varepsilon_{\text {rec }}^{\gamma \gamma}$ & 0.358 & 0.553 & 0.740 \\
Stat Err & \pm 0.003 & \pm 0.005 & \pm 0.018 \\
Syst Err & \pm 0.007 & \pm 0.029 & \pm 0.063 \\
\hline
\end{tabular}

TABLE 6.6. TStnElectron reconstruction efficiency averaged for different $E_{\mathrm{T}}$ cuts and $|\eta|<1.1$. The full CDF simulation and reconstruction software was used.

\begin{tabular}{lccc}
\hline$E_{\mathrm{T}}$ cut $(\mathrm{GeV})$ & 2.0 & 2.5 & 5.0 \\
\hline$\varepsilon_{\text {rec }}^{e^{+} e^{-}}$ & 0.330 & 0.508 & 0.802 \\
Stat Err & \pm 0.006 & \pm 0.007 & \pm 0.017 \\
Syst Err & \pm 0.009 & \pm 0.016 & \pm 0.037 \\
\hline
\end{tabular}




\subsection{Exclusive Filter}

The exclusive filter selected only those events that include rapidity gaps by requiring a void of particles in all subdetectors up to a rapidity $\eta$ of 7.4 . We selected only events that have no other activity in the whole detector than our two EM shower signals.

Exclusivity Cuts. The various sub-detectors consist of the central and plug calorimeters, the Miniplug detector as well as the BSC counters and the CLC. We divided the Central and Plug calorimetry into sections as shown in Table 6.7.

TABLE 6.7. Definition of detector regions, with calorimeter tower numbers.

\begin{tabular}{lcccc}
\hline \multicolumn{5}{c}{ Definition of calorimeter sections } \\
Section & Towers & iEta east & iEta west & $|\eta|$ \\
\hline Central EM/HAD Cal.: & $0-5$ & $20-25$ & $26-31$ & $0-0.66$ \\
End Wall EM/HAD Cal.: & $6-11$ & $14-19$ & $32-37$ & $0.66-1.32$ \\
Mid Plug Cal.: & $12-17$ & $8-13$ & $38-43$ & $1.32-2.11$ \\
Forward Plug Cal.: & $18-21$ & $4-7$ & $44-47$ & $2.11-3.64$ \\
Miniplug Cal.: & $22-25$ & $0-3$ & $48-51$ & $3.6-5.2$ \\
\hline CLC & & & & $3.7-4.7$ \\
BSC1 - BSC3 & & & $5.4-7.4$ \\
\hline
\end{tabular}

For all sub-detectors or calorimeter sections, cuts were determined that separate noise from the signal. Whenever there was a hit with a larger $E_{T}$ or higher ADC counts compared to the cut values in any PMT of the above sub-detectors, we declared the event as non exclusive and removed it from the candidate list. The cut values can be found in Table 6.8. This was done in the same way as in the previous study of exclusive $e^{+} e^{-}$production by the CDF collaboration [10].

Zero-bias data ${ }^{1}$ was used to obtain the cut values. The data was split into an interaction and a non-interaction sample. Event candidates for the non-interaction sample had to pass all the following cuts.

- No tracks (CDF-track with $p_{T}>200 \mathrm{MeV}$ )

- No CLC hit (<150 ADC counts)

- No Muon stub (track in the Muon detectors)

The interaction sample is composed of all events that did not pass those cuts, and includes nearly all events that had more than one inelastic collision. In Figure 6.5 to Figure 6.10 the maximum $E_{T}$ distributions for the interaction and non-interaction samples of the zero-bias data are shown for the calorimetry sections up to the Miniplug

\footnotetext{
${ }^{1}$ This data consists of random events only triggered on a bunch crossing.
} 
TABLE 6.8. Exclusive cut values for different sub-detectors. If any detector component (e.g. a PMT of the EM calorimeter) had a signal above the cut value the event was rejected.

\begin{tabular}{ll}
\hline \multicolumn{2}{c}{ Exclusive filter cuts } \\
\hline Central EM Calorimeter $\left(E_{T}\right):$ & $80 \mathrm{MeV}$ \\
Central HAD Calorimeter $\left(E_{T}\right):$ & $200 \mathrm{MeV}$ \\
End Wall EM Calorimeter $\left(E_{T}\right):$ & $80 \mathrm{MeV}$ \\
End Wall HAD Calorimeter $\left(E_{T}\right):$ & $200 \mathrm{MeV}$ \\
Mid Plug Calorimeter $\left(E_{T}\right):$ & $80 \mathrm{MeV}$ \\
Forward Plug Calorimeter $\left(E_{T}\right):$ & $30 \mathrm{MeV}$ \\
Miniplug Calorimeter $\left(E_{T}\right):$ & $5 \mathrm{MeV}$ \\
BSC1 (ADC): & $400 \mathrm{counts}$ \\
BSC2 (ADC): & 300 counts \\
BSC3 (ADC): & 400 counts \\
CLC (Sum of west and east) (ADC): & 6300 \\
\hline
\end{tabular}

detector. Some interaction events below the cut were expected, as inelastic events can have rapidity gaps (for example diffractive physics events). In Figure 6.11 to Figure 6.13 the maximum ADC count distributions for both samples are shown for the BSC counters.

The calorimeter towers in the central and plug regions that had been hit by the signal EM showers were excluded from the filter. Further we used a Spike Killer algorithm in order to ignore isolated energy $P M T$ spikes $^{2}$ in the calorimetry. To be more precise, we used this code for energy deposits $<0.5 \mathrm{GeV}$ down to the noise level as the CDF calorimeter reconstruction software uses it by default for $>0.5 \mathrm{GeV}$. In Table 6.9 the numbers of events that passed each sequential exclusivity cut are listed, starting from the sample of triggered events. All listed steps are additive.

Exclusive Efficiency. The efficiency of this exclusive filter is the probability that an exclusive event will pass our exclusive cuts, and depends on the instantaneous luminosity of each bunch crossing. Any additional activity due to pileup will destroy the clean environment of an exclusive state. Such events will therefore not pass the exclusivity filter. The efficiency factor was applied to the delivered luminosity to account for the requirement of no pileup. To determine the efficiency we used the method described in the earlier CDF study on the exclusive electron-positron

\footnotetext{
${ }^{2} \mathrm{~A}$ calorimeter tower is equipped with two separate PMT's. In case one is malfunctioning and returning a non existing signal (spike) one can correct for that using the other PMT. The software algorithm removing those fake signals is called Spike Killer. Developed for earlier CDF analyses it proved to be very efficient $[\mathbf{1 3 1}]$.
} 

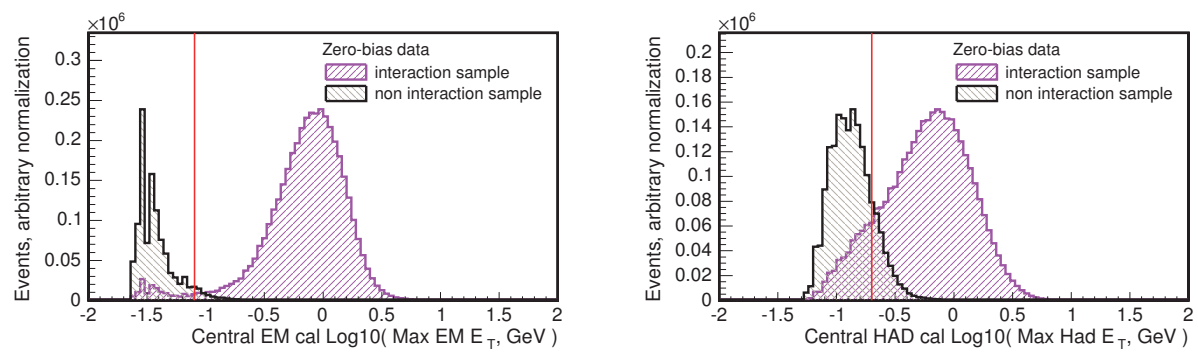

FIgURE 6.5. Activity in the central EM calorimeter for the interaction and non-interaction sample (left). Activity in the central HAD calorimeter for the interaction and non-interaction sample (right). The vertical lines show the exclusivity cuts.
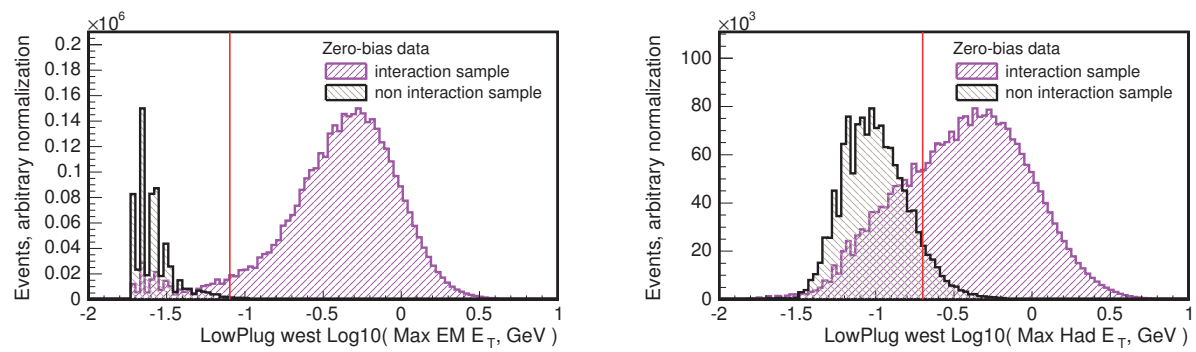

FiguRE 6.6. Activity in the west end wall EM calorimeter for the interaction and non-interaction sample (left). Activity in the west end wall HAD calorimeter for the interaction and non-interaction sample (right). The vertical lines show the exclusivity cuts.
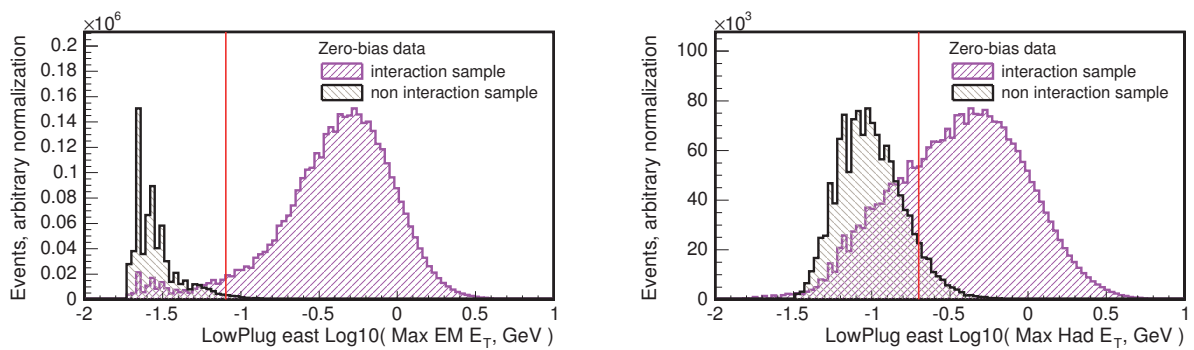

Figure 6.7. Activity in the east end wall EM calorimeter for the interaction and non-interaction sample (left). Activity in the east end wall HAD calorimeter for the interaction and non-interaction sample (right). The vertical lines show the exclusivity cuts. 

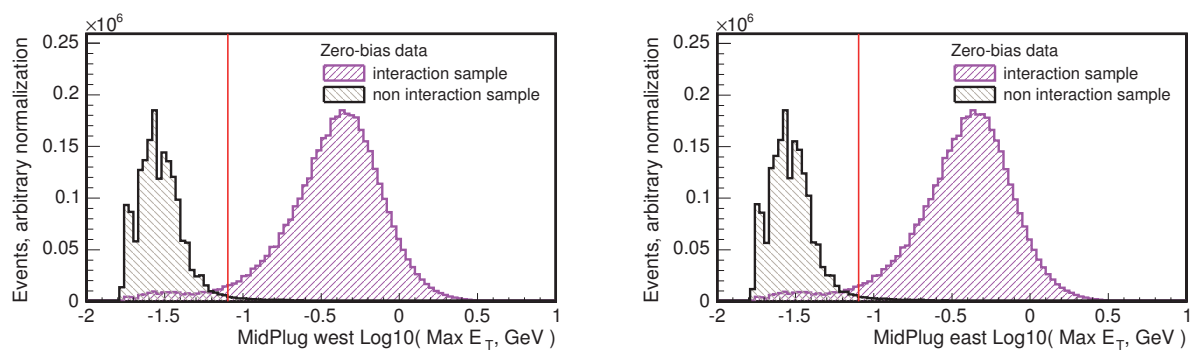

FIgURE 6.8. Activity in the west mid plug calorimeter for the interaction and non-interaction sample (left). Activity in the east mid plug calorimeter for the interaction and non-interaction sample (right). The vertical lines show the exclusivity cuts.
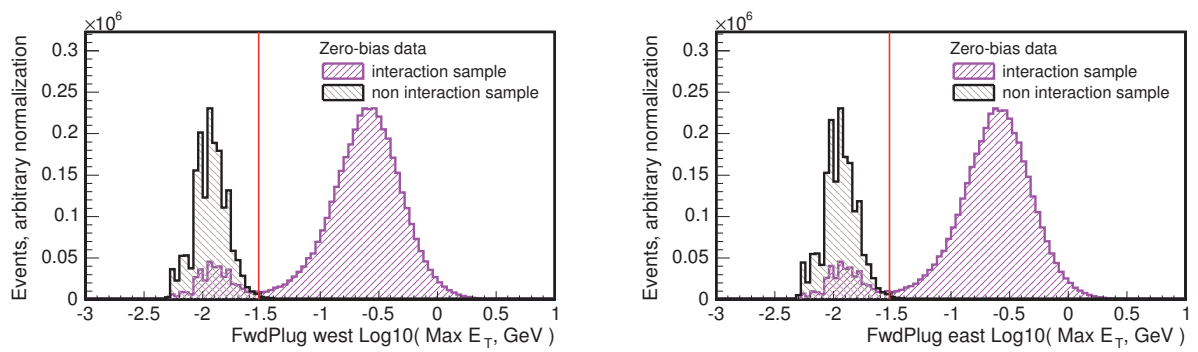

Figure 6.9. Activity in the west forward plug calorimeter for the interaction and non-interaction sample (left). Activity in the east forward plug calorimeter for the interaction and non-interaction sample (right). The vertical lines show the exclusivity cuts.
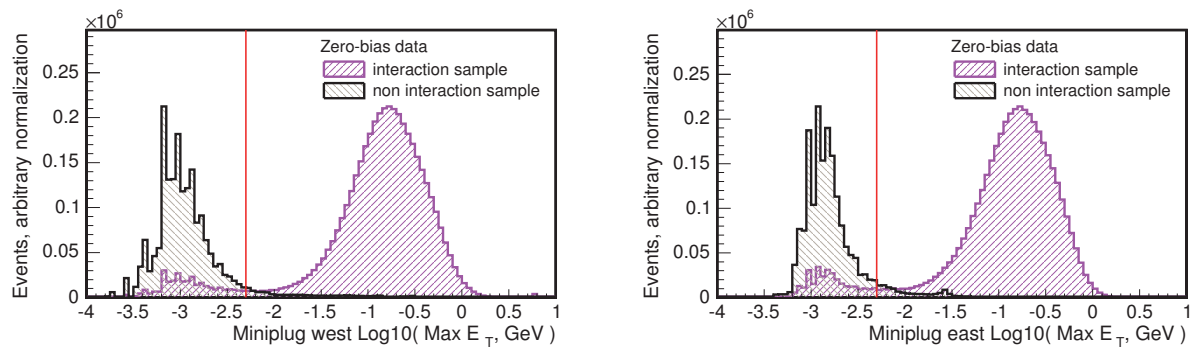

Figure 6.10. Activity in the west Miniplug calorimeter for the interaction and non-interaction sample (left). Activity in the east Miniplug calorimeter for the interaction and non-interaction sample (right). The vertical lines show the exclusivity cuts. 

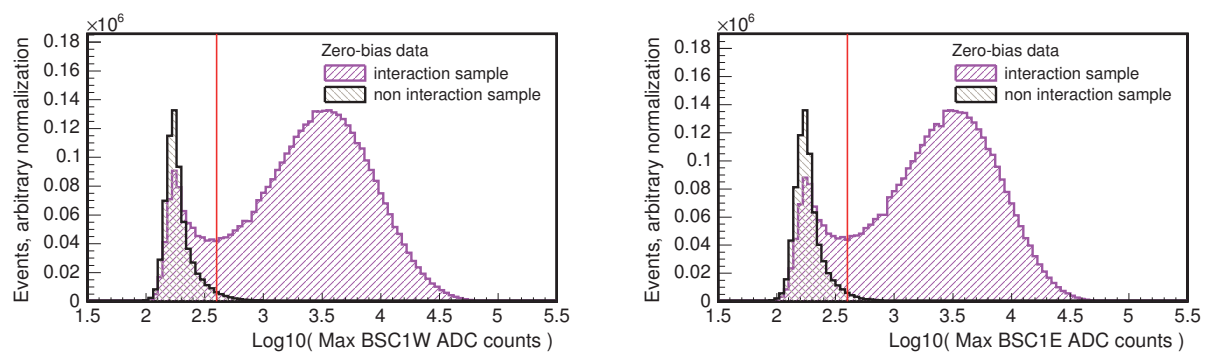

Figure 6.11. Activity in the west BSC1 for the interaction and noninteraction sample (left). Activity in the east BSC1 for the interaction and non-interaction sample (right). The vertical lines show the exclusivity cuts.
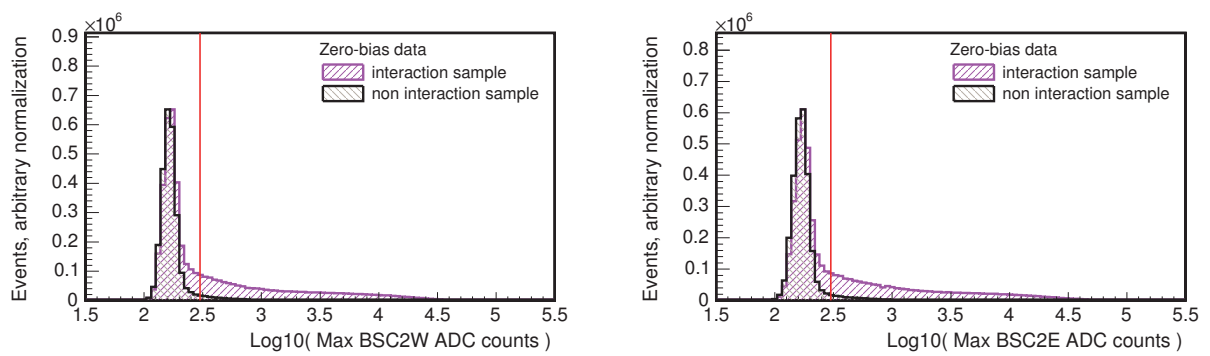

Figure 6.12. Activity in the west BSC2 for the interaction and noninteraction sample (left). Activity in the east BSC2 for the interaction and non-interaction sample (right). The vertical lines show the exclusivity cuts.
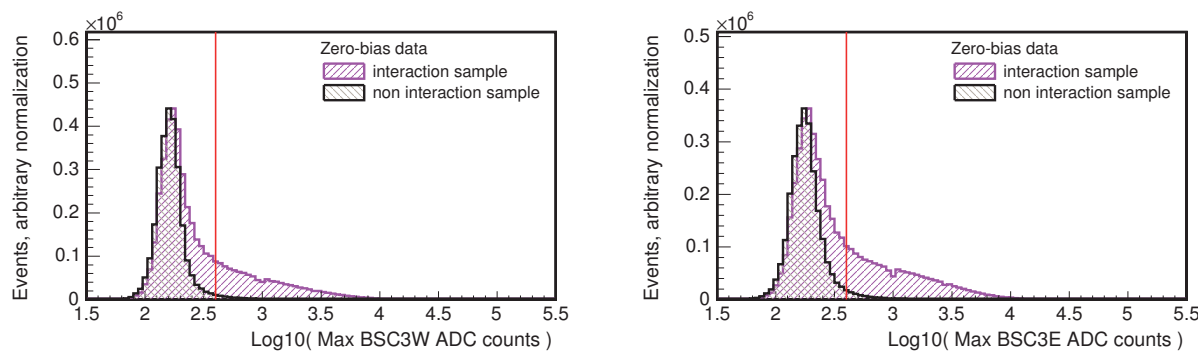

Figure 6.13. Activity in the west BSC3 for the interaction and noninteraction sample (left). Activity in the east BSC3 for the interaction and non-interaction sample (right). The vertical lines show the exclusivity cuts. 
TABLE 6.9. Number of events that passed each exclusive cut of the different sub-detectors.

\begin{tabular}{lrr}
\hline \multicolumn{3}{c}{ Number of events after exclusive cuts } \\
\hline Cut & $|\eta|$ coverage & Number of events \\
\hline Trigger: & & $200,143,239$ \\
Preselection (2EM central+plug): & $5.4-7.4$ & $93,976,483$ \\
Empty BSC counters (all): & $3.6-5.2$ & $39,099,062$ \\
Empty Miniplug and CLC: & $2.11-3.64$ & 136,914 \\
Empty Forward Plug Calorimeter: & 13,974 \\
Empty Mid Plug Calorimeter: & $1.32-2.11$ & 5,254 \\
Empty Low Plug Calorimeter: & $0.66-1.32$ & 1,359 \\
Empty Central Calorimeter: & $0.0-0.66$ & 421 \\
\hline
\end{tabular}

pair production at CDF [10]. As the exclusive efficiency is a function of the bunch luminosity we needed first to retrieve the bunch weight from the database for each run. The bunch weight was taken to be constant during a run, motivated by the former CDF study. They looked at bunch luminosities at the beginning and at the end of a prolonged data taking run and found that the bunch weight stays constant within $5 \%$. The instantanous bunch luminosity is,

$$
\mathcal{L}_{\text {bunch, inst }}=\frac{\mathcal{L}_{\text {inst }}}{\# \text { Bunches }} \times \text { Bunch weight. }
$$

The Tevatron was run with 36 bunches of protons and of antiprotons. For each bunch crossing the exclusive efficiency is defined as the probability that an exclusive event is not killed by another inelastic collision. If $\sigma_{\text {inel, det }}$ is the inelastic cross section detected by the detector (det) and $L_{\mathrm{b}}$ the individual bunch luminosity (integrated), the probability of having no additional interaction to fill the rapidity gap is

$$
P(0)=a e^{-\sigma_{\text {inel, det }} L_{\mathrm{b}}} .
$$

If the cuts are set correctly above the noise we should have $a=1.0$. If the detector has complete coverage for inelastic collisions, and is "clean", e.g. not having signals related to a previous bunch crossing, the distribution will be a good exponential and $\sigma_{\text {inel, det }}=\sigma_{\text {inel }}$. These are good checks of the procedure. Intuitively the exclusive efficiency $\varepsilon_{\text {exc }}$ is the number of observed exclusive events that passed our exclusive cuts divided by the total number of real exclusive events,

$$
\varepsilon_{\mathrm{exc}}=\frac{\# \text { Events }_{\mathrm{exc}}^{\mathrm{obs}}}{\# \text { Events }_{\mathrm{exc}}^{\mathrm{real}}} .
$$


The effective luminosity can be obtained via

$$
\mathcal{L}_{\text {eff }}=\int \varepsilon_{\text {exc }} \mathcal{L}_{\text {bunch }} \mathrm{dt} .
$$

It was shown in $[\mathbf{1 0}]$ that the exclusive efficiency can be determined using zero-bias data,

$$
\varepsilon_{\text {exc }}=\frac{\# \text { Event }_{\text {exc }}^{\text {obs }}}{\# \text { Events }_{\text {exc }}^{\text {real }}}=\frac{\# \text { Events }_{\text {zerobias }}^{\text {pass }}}{\# \text { Events }_{\text {zerobias }}^{\text {all }}} .
$$

In Figures 6.14 to 6.16 the exclusive efficiencies for each data taking period are shown as a function of the bunch luminosity. The zero-bias data used covers the same data periods that we used for the photon-pair search. Also the same Good Run lists were applied that we used for our event selection due to the direct dependence of the exclusive efficiency on the bunch luminosity.

The slopes and the intercepts at zero bunch luminosity of the exponential fits to the exclusive efficiencies for each data taking period are shown in Figure 6.17.

Initially we found a non-exponential behavior in the exclusive efficiency for low bunch luminosities for data taking period 12 as shown in Figure 6.18 on the left. This caused a different slope and intercept for run period 12 which can be seen in Figure 6.19. We saw that period 12, in the case where we used the official Good Run list ${ }^{3}$, did not show the expected behavior. We found out that this was caused by the Miniplug detector. When we ignored this device and used instead the CLC detector, which covers almost the same angular region as the Miniplug, we saw a good exponential behavior of the exclusive efficiency as shown in Figure 6.18. In order to find out what caused these problems in the Miniplug detector, we reviewed the data taking in period 12 run by run. Using non-interaction zero bias data in the same manner as for finding good exclusivity cuts we requested for such runs that had no activity in any detector except the Miniplug, as we suspected some hot towers or readout problems. We found several runs within Period 12 that had large signals in the Miniplug when none were expected. Figure 6.20 shows as an example the result of zero bias non-interaction data in the Miniplug for two problematic runs. We carefully removed all data taking runs from the official Good Run list that showed a misbehaving Miniplug detector, and obtained the results shown above (see Figure 6.16).

The method of how we measured the overall exclusive efficiency is illustrated in Figure 6.21. As an example we only show the figure for data taking period 9; it is similar to all the other periods. The violet histogram represents the bunch luminosity distribution for all zero-bias events selected in our data taking period. The black histogram shows the bunch luminosity weighted by the exclusive efficiency, i.e. the

\footnotetext{
${ }^{3}$ This Good Run list is corrected for all officially known problems of the used subdetectors.
} 

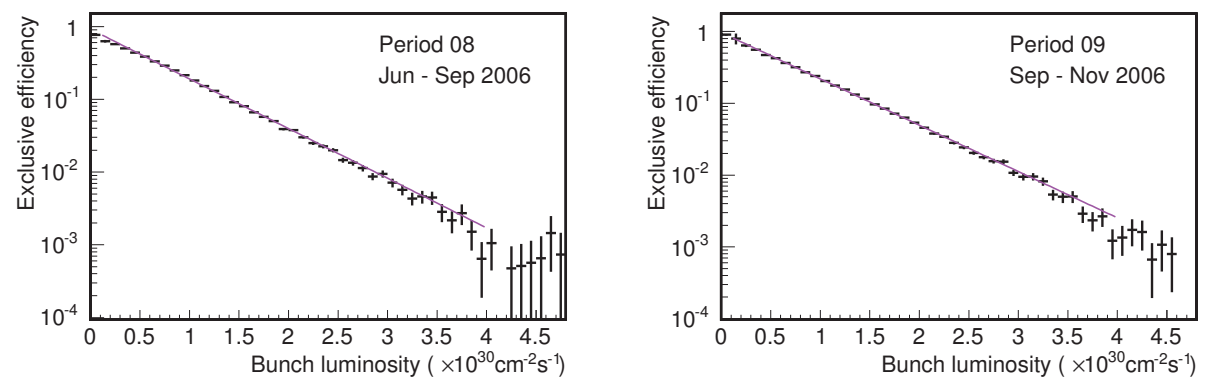

FIgURE 6.14. Exclusive efficiency as a function of the bunch luminosity for data taking period 8 (left) and period 9 (right).
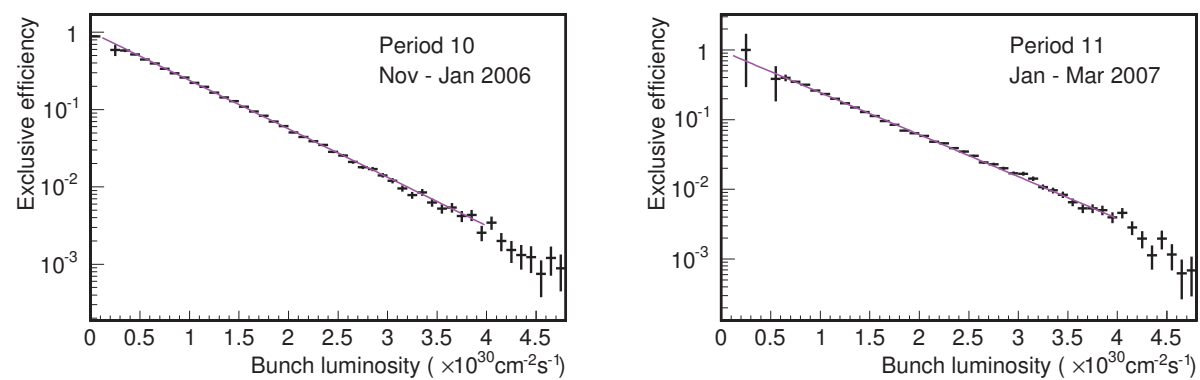

FiguRE 6.15. Exclusive efficiency as a function of the bunch luminosity for data taking period 10 (left) and period 11 (right).
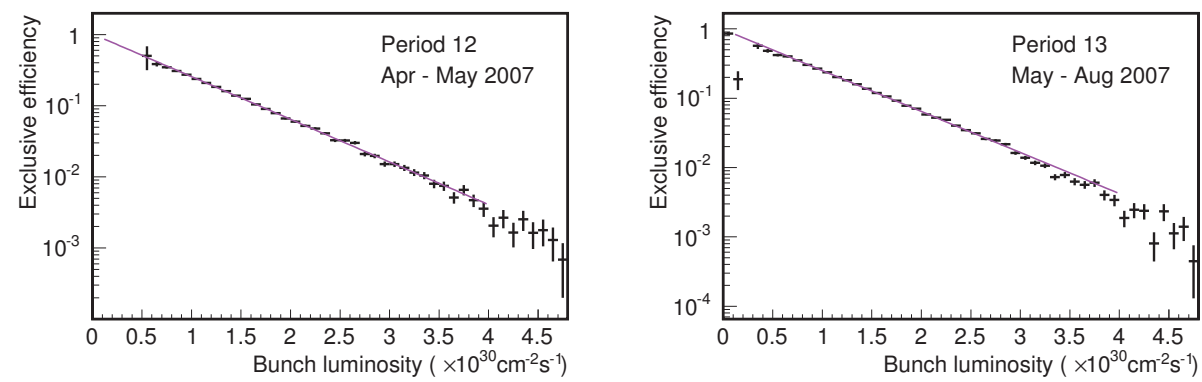

FiguRE 6.16. Exclusive efficiency as a function of the bunch luminosity for data taking period 12 (left) and period 13 (right). 

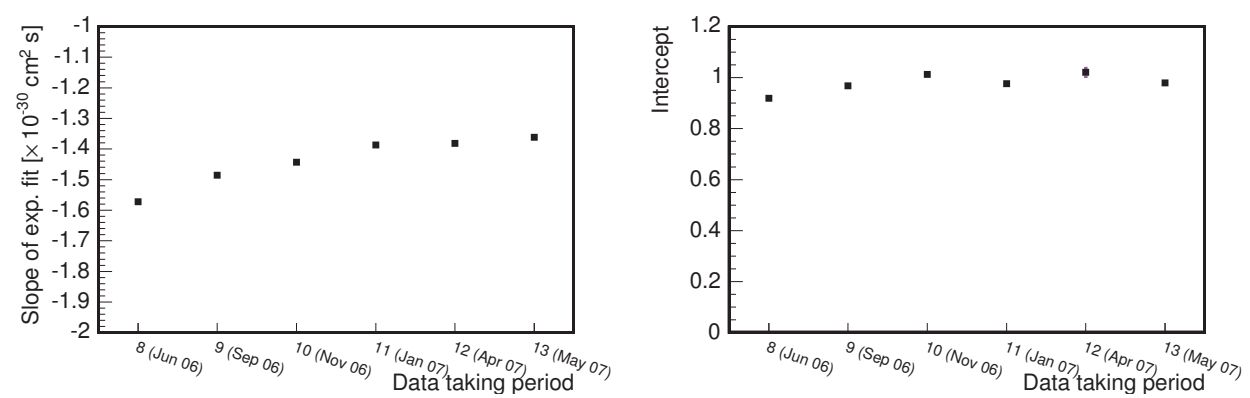

Figure 6.17. Slope of the exponential fit of the exclusive efficiencies for data taking period 8 to 13 (left). The intercept of the fit function at zero luminosity for period 8 to 13 (right). Note, the magnitude of the statistical errors are of the order of the marker size.
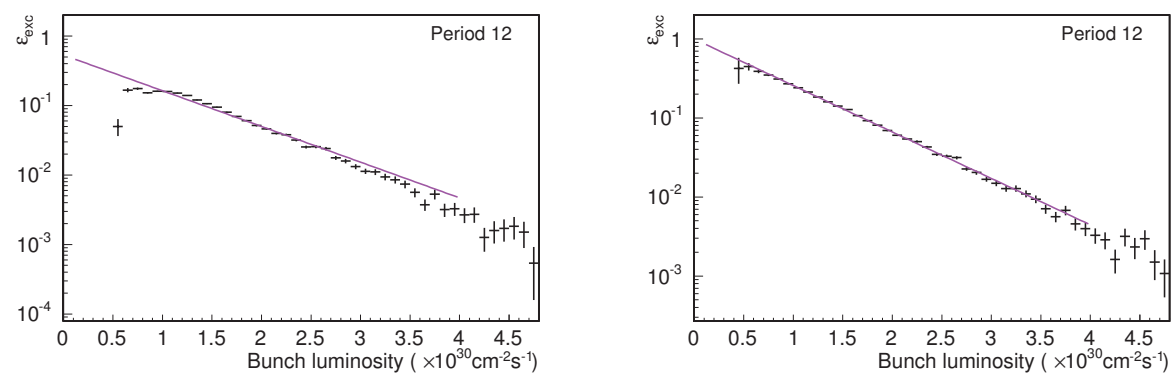

Figure 6.18. Exclusive efficiency as a function of the bunch luminosity for data taking period 12 with (left) and without (right) using the Miniplug detector.

subset of events that passed our exclusive criteria. Dividing the black histogram by the violet histogram we obtained the overall exclusive efficiency. Due to large statistics the statistical error is negligible. In Table 6.10 all values of interest for the exclusive efficiency estimate are listed.

The uncertainty of the effective luminosity is dominated by the standard uncertainty of the luminosity of about $6 \%$ according to [10]. To estimate the systematic uncertainty we calculated the cross section for each data-taking period from the slope of the fitted exponential to the exclusive efficiency curve. The obtained results specified for the different run periods are summarized in Table 6.10. We measured an average "total 

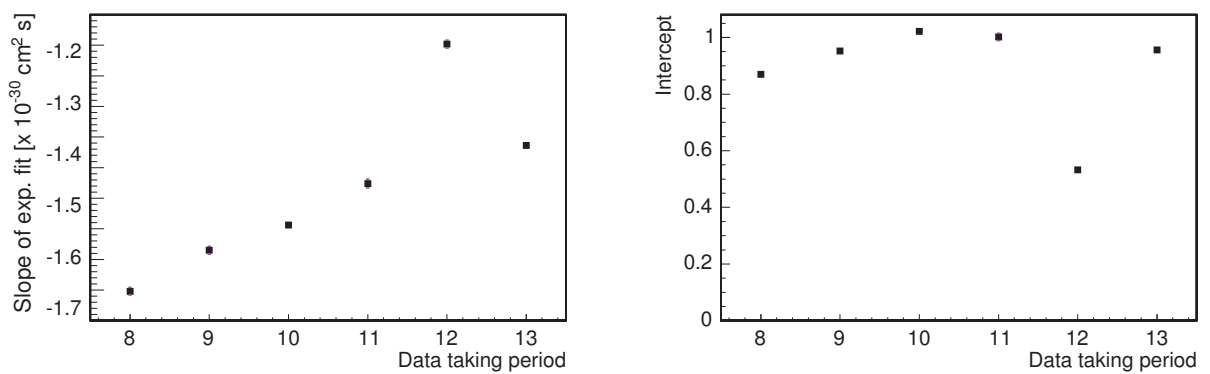

Figure 6.19. Slope of the exponential fit of the exclusive efficiencies for data taking period 8 to 13 including the Miniplug (left). The intercept of the fit function at zero luminosity for period 8 to 13 using the Miniplug (right). Note the magnitude of the statistical errors are of the order of the marker size.
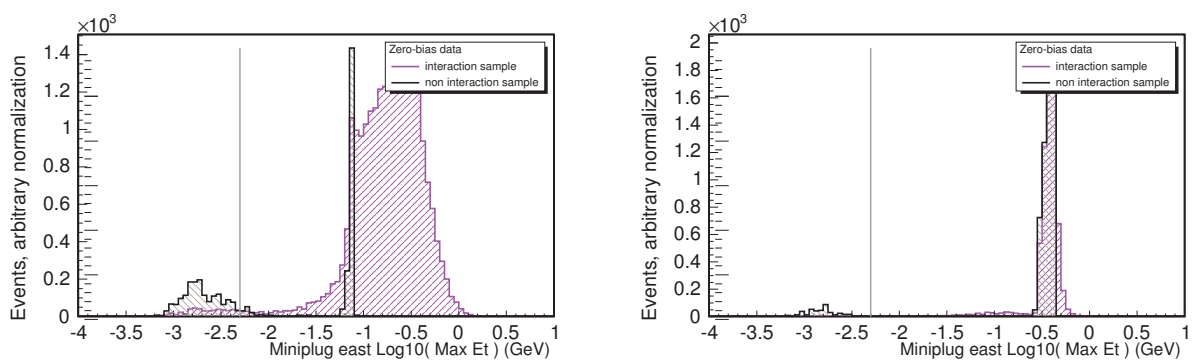

Figure 6.20. Activity in the East Miniplug calorimeter for the interaction and non-interaction sample for run 239230 (left) and for run 241616 (right). Note the large signal in case of the non-interaction sample. This is a sign for hot towers or readout problems.

inelastic" cross section of $67 \pm 4 \mathrm{mb}^{4}$. The $5.6 \%$ error on the cross section was taken as a systematic error for our exclusive efficiency estimate, that is $\varepsilon_{\text {exc. }}=0.068 \pm 0.004$. The effective luminosity was $\mathcal{L}_{\text {eff }}=\varepsilon_{\text {exc }} \times \mathcal{L}_{\text {int }}=0.068 \times 1.11 \mathrm{fb}^{-1}=(74 \pm 4) \mathrm{pb}^{-1}$.

\subsection{Post-selection of EM Exclusive Events}

For a further selection of exclusive $\gamma \gamma$ candidates we required exactly 2 EM objects in a pseudorapidity region of $|\eta|<1.0$. We first used 1.8 but found many ambiguous events that showed signs of being electron events without having reconstructed tracks. We then

\footnotetext{
"We call it here "total inelastic" cross section even though we do not have a complete detection coverage for inelastic collisions.
} 


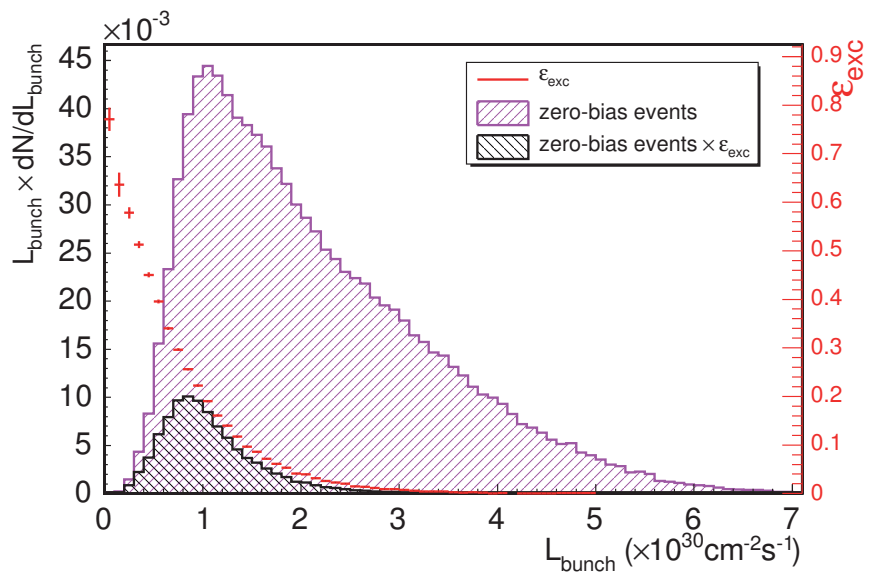

FiguRE 6.21. Histograms of the bunch luminosity of zero-bias data (violet) as well as the bunch luminosity of zero-bias events that passed the exclusive cuts (black). The red curve shows the obtained exclusive efficiency as a function of the bunch luminosity (scale on the right side). The overall exclusive efficiency is obtained by dividing the black histogram by the violet one.

TABLE 6.10. List of values for the integrated luminosity, the slope and the intercept of the fit to the exclusive efficiency curve, the inelastic cross section, the exclusive efficiency and the effective luminosity $\left(\mathrm{pb}^{-1}\right)$ split between the different data taking periods.

\begin{tabular}{ccccccc}
\hline Period & $\mathcal{L}_{\text {int }}\left[\mathrm{pb}^{-1}\right]$ & Slope $\left[10^{30} \mathrm{~cm}^{2} \mathrm{~s}\right]$ & Intercept & $\sigma[\mathrm{mb}]$ & $\varepsilon_{\text {excl }}$ & $\mathcal{L}_{\text {eff }}\left[\mathrm{pb}^{-1}\right]$ \\
\hline 8 & 131 & $-1.57 \pm 0.01$ & $0.92 \pm 0.01$ & 73 & 0.0875 & 11.4 \\
9 & 147 & $-1.49 \pm 0.01$ & $0.97 \pm 0.01$ & 69 & 0.0787 & 11.6 \\
10 & 238 & $-1.44 \pm 0.01$ & $1.01 \pm 0.01$ & 67 & 0.0680 & 16.2 \\
11 & 212 & $-1.39 \pm 0.01$ & $0.98 \pm 0.01$ & 64 & 0.0540 & 11.4 \\
12 & 121 & $-1.38 \pm 0.01$ & $1.02 \pm 0.02$ & 64 & 0.0521 & 6.28 \\
13 & 266 & $-1.36 \pm 0.01$ & $0.98 \pm 0.01$ & 63 & 0.0655 & 17.4 \\
\hline Tot: & 1114 & & & $66.9 \pm 4$ & $0.0680 \pm 0.004$ & 74.3 \\
\hline
\end{tabular}

restricted the pseudo-rapidity region to $|\eta|<1.0$. The $2 \mathrm{EM}$ objects with a minimum $E_{T}$ of $2 \mathrm{GeV}$ were additionally filtered by a cut on the hadronic/electromagnetic energy ratio (HadEM) of $0.055+0.00045 * E_{\text {Corr }}$ for the central region and 0.05 for the plug region. $E_{\text {corr }}$ is the offline corrected energy. For the final sample we chose an $E_{T}$ cut of $2.5 \mathrm{GeV}$, but we also list partly information for $E_{T}$ cuts of 2.0 and $5.0 \mathrm{GeV}$. 
TABLE 6.11. Cuts for EM object selection.

\begin{tabular}{ll}
\hline \multicolumn{2}{c}{ Loose EM object cuts } \\
\hline Pseudorapidity & $|\eta|<1.0$ \\
Transverse energy & $E_{\mathrm{T}}>2.0 \mathrm{GeV}$ \\
HAD/EM ratio (central) & $0.055+0.00045 * E_{\text {Corr }}$ \\
Separation angle & $|\pi-\Delta \phi|<0.6$ \\
EoverP $^{5}$ & $E / p<2.0$ \\
\hline
\end{tabular}

Additionally we cut on the azimuthal separation angle in form of $|\pi-\Delta \phi|$, to ensure back-to-back events. The usual isolation cut to ensure single photons or electrons without other activity in the nearby surroundings was not used as it is completely covered by the exclusive filter. Also the $\chi^{2}$ cut on the shape of the CES showers was not used. Usually it is applied to distinguish photons from neutral pions and removing the latter; $\pi^{0}$ in our low energy range do not give broad showers as the two photon showers cannot merge. In the case of higher energetic objects it is different and such a cut is very useful. Possible $\pi^{0}$ background will be studied in detail later in the background discussion. After the cuts, common to both electron and photons, had been applied we were left with 180 events for further studies. Additionally a cut of $E / p$ was applied to the electron-positron pair candidate sample (separated later) to ensure a good match of the measured track $p_{T}$ to the calorimeter energy deposit $E$. The complete list of cuts can be found in Table 6.11. Before we separate the electron-positron pair and photon pair events from the exclusive sample we discuss the efficiencies for post-selecting electrons and photons.

Post-identification Efficiencies. The efficiencies for selecting photons or electrons that passed the post-selection filter were obtained by using unbiased and unfiltered low $p_{T}$ electrons (probe electrons). By unbiased we mean that the electrons should not be influenced by any trigger cuts or other selection criteria. We divided the number of probe electrons that pass the post-selection cuts with the total amount of probe electrons, which gives us the efficiency,

$$
\varepsilon_{\text {post-id }}=\frac{\# \text { EM objects }_{\text {post }- \text { id }}}{\# \text { EM objects }}
$$

By using electrons from $J / \Psi$ meson decays we obtained high quality probe electrons with very low background. We retrieved a clean sample of events containing $J / \Psi$ events using the SUSY_DILEPTON dataset (edil0i/j) ${ }^{6}$. A single electron trigger with a $4 \mathrm{GeV}$

\footnotetext{
${ }^{5}$ This cut is only applied to the electron sample.

${ }^{6}$ The SUSY_DILEPTON dataset is mainly used for beyond the SM physics searches. It contains an enhanced selection of dilepton final states.
} 
threshold was used to select events including $J / \Psi$. Only events with two or three good electrons were taken. To remove this trigger bias we asked for one electron to have a Level 3 EM Object match and pass all trigger cuts. We now searched for an unbiased probe electron with opposite charge to the trigger electron. An invariant mass of the electron pair laying within the tight $J / \Psi$ mass window between 2.9 and $3.3 \mathrm{GeV}$ was required to ensure a clean and low background event sample. Furthermore, the probe electron had to be separated from the trigger electron by $\Delta R=\sqrt{\Delta \eta^{2}+\Delta \phi^{2}} \geq 0.4$.

Now we used the probe electrons to test our quality cuts and obtain the efficiency. Using $J / \Psi$ we could only determine our identification efficiency down to $E_{T}$ of $4 \mathrm{GeV}$ due to low statistics. To extend the range down to $2 \mathrm{GeV}$ we used simulated $e^{+} e^{-}$ pair events produced by the LPAIR Monte Carlo program. The events were processed through the full CDF detector simulation and reconstruction software. For the LPAIR sample no strict selection criteria were required. It is enough to ask for at least one reconstructed low level TStnElectron (reconstruction level electron).

In particular we were interested in the HadEm cut efficiency. For electrons we were additionally interested in the $E / p$ cut. The $|\pi-\Delta \phi|$ cut to ensure back-to-backness of the candidates, does not apply to single photons or electrons but to the whole event. However, as we will see later when comparing data with Monte Carlo simulated events, this cut could be taken in fact as $100 \%$ efficient.

In Figure 6.22 we see the HadEm cut efficiency over $E_{T}$, pseudorapidity $\eta$ and azimuthal angle $\phi$ of the probe electron. One can see that the HadEm cut efficiency versus electron $E_{T}$ of the $J / \Psi$ sample agrees with the Monte Carlo sample result in the overlapping interval. The efficiency versus $\eta$ and $\phi$ is flat for both samples. In both, the $\eta$ and $\phi$ plot, the $J / \Psi$ sample seems to be less affected by the cut. The $E_{T}$ range of the Monte Carlo sample goes down to $2 \mathrm{GeV}$ (compared to $4 \mathrm{GeV}$ for the $J / \Psi$ sample) where we have a slight drop down of the efficiency for low $E_{T}$. The efficiency integrated over $E_{T}$ is therefore visibly lower in those plots. It was not intended to be a detailed comparison of Monte Carlo and data, but to show that it was justified within our limits to use the Monte Carlo sample for estimating the efficiency down to a transverse energy of $2 \mathrm{GeV}$.

The efficiency of the $E / p$ selection used to ensure good electron-positron pair candidates was obtained similarly to the HadEm cut efficiency. Using here only the simulated electron-pair sample we counted the number of probe electrons that passed the filter cut. Dividing this number by the total amount of probe electrons gave us the efficiency. The resulting EoverP selection efficiency as a function of $E_{T}$ is shown in Figure 6.23. The identification efficiency results can be found in Table 6.12, where the electron identification efficiency is a combination of the HadEm and EoverP cut 

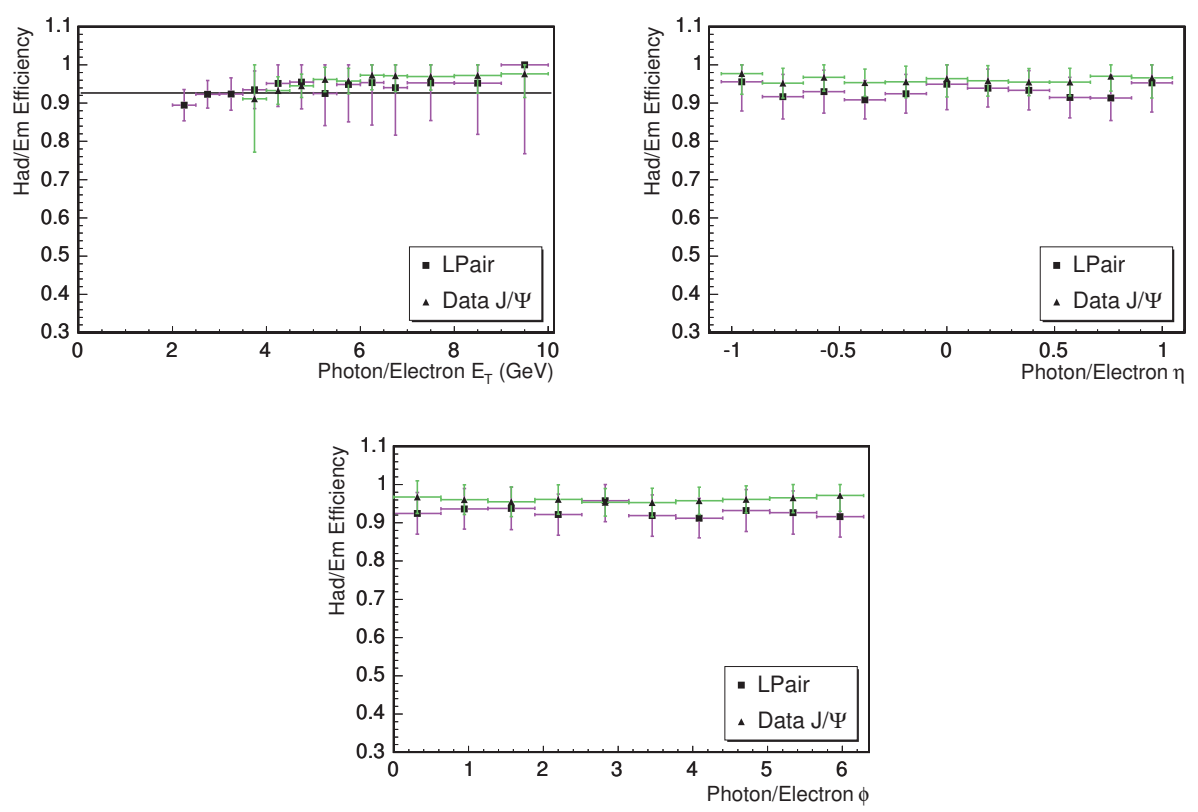

Figure 6.22. HadEm cut efficiency versus $E_{T}, \eta$ and $\phi$ for electrons/photons. Unbiased electrons from data $J / \Psi$ decays have been used as well as a exclusive $e^{+} e^{-}$LPAIR Monte Carlo sample to extend the study to lower $E_{T}$. For the Monte Carlo sample full CDF simulation and reconstruction software has been used.

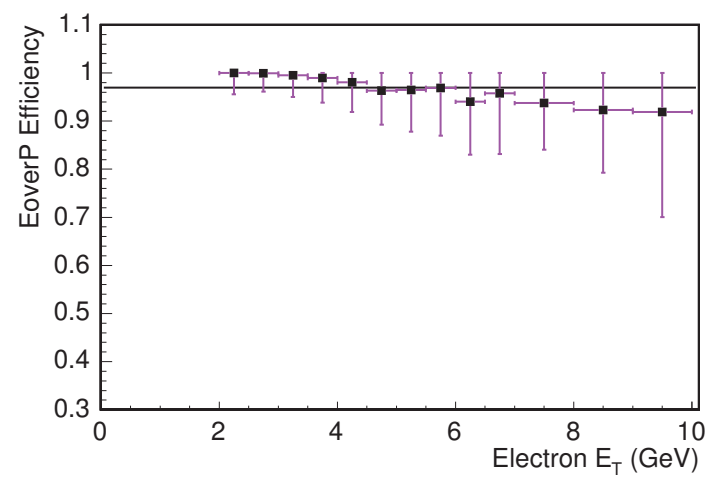

Figure 6.23. Efficiency of the $E / p$ cut for electrons. An exclusive Monte Carlo $e^{+} e^{-}$sample was used, produced by the LPAIR program in connection with a full CDF detector simulation and reconstruction. 
TABle 6.12. Post-identification efficiency for photons and electrons averaged over the kinematic range of $\left(E_{T}>2.5 \mathrm{GeV},|\eta|<1.1\right)$.

\begin{tabular}{lc}
\hline \multicolumn{2}{c}{ Photon/Electron ID efficiency } \\
\hline$\varepsilon_{\text {id, photon }}$ & $0.927 \pm 0.017$ (stat) \pm 0.013 (syst) \\
$\varepsilon_{\text {id, electron }}$ & $0.912 \pm 0.017$ (stat) \pm 0.013 (syst) \\
\hline
\end{tabular}

TABLE 6.13. Number of exclusive $e^{+} e^{-}$pairs split into different data taking periods.

\begin{tabular}{c|ccccc}
\hline \multirow{2}{*}{ Period } & \multicolumn{2}{|c}{$E_{\mathrm{T}}>2 \mathrm{GeV}$} & \multicolumn{2}{c}{$E_{\mathrm{T}}>2.5 \mathrm{GeV}$} & \multicolumn{2}{c}{$E_{\mathrm{T}}>5 \mathrm{GeV}$} \\
\cline { 2 - 6 } & $e^{+} e^{-}$ & $\div \mathcal{L}_{\text {eff }}[\mathrm{pb}]$ & $e^{+} e^{-}$ & $\div \mathcal{L}_{\text {eff }}[\mathrm{pb}]$ & $e^{+} e^{-}$ \\
\hline 8 & 6 & 0.52 & 4 & 0.35 & 1 \\
9 & 6 & 0.52 & 5 & 0.43 & 3 \\
10 & 7 & 0.43 & 7 & 0.43 & 4 \\
11 & 9 & 0.79 & 8 & 0.70 & 2 \\
12 & 2 & 0.32 & 2 & 0.32 & 0 \\
13 & 9 & 0.52 & 8 & 0.46 & 0 \\
\hline Tot: & 39 & 0.52 & 34 & 0.46 & 10 \\
\hline
\end{tabular}

efficiency. The systematic errors were estimated by varying the values of the HadEm and EoverP cuts conservatively by $10 \%$.

\section{Exclusive Electron-Positron Pair Control Channel}

\subsection{Selection of Candidates}

The final selection of exclusive $e^{+} e^{-}$events from the sample of exclusive events was done by requiring exactly one single charged particle track per EM object. The track had to have a transverse momentum $p_{T}>1 \mathrm{GeV}$. Additionally we filtered for a good match of the track $p_{T}$ with the reconstructed calorimeter energy of the EM object, $E / p<2.0$, as already introduced above. This gave us our final samples of clean electron-positron pair candidates. None of the pairs have the same sign of charge. Details can be found in Table 6.13 for different $E_{T}$ cuts.

2.1.1. Tracking Efficiency. In the central region the CDF detector is very efficient in tracking with its silicon vertex detector and the central outer tracker. Using a clean $Z \rightarrow e^{+} e^{-}$sample we could probe the tracking efficiency for electrons. The events had been selected by a trigger with no track requirement. Additionally the 


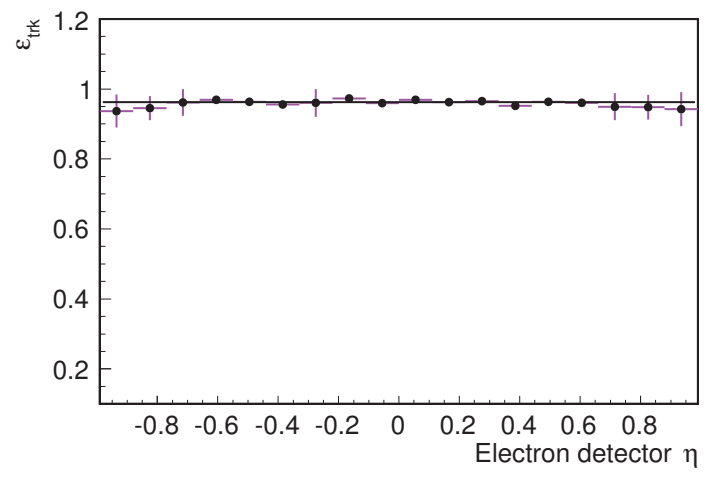

FiguRE 6.24. Tracking efficiency for central electrons versus pseudorapidity $\eta$.

events had to have exactly two electron candidates within a invariant mass range from 80 to $100 \mathrm{GeV}$. If one electron had a track then the other electron was used as a probe electron. The results can be seen in Figure 6.24 with the tracking efficiency plotted versus the detector pseudorapidity $\eta$. In the central region the distribution is flat. Therefore we fitted a constant to the curve to obtain the tracking efficiency for $|\eta|<1.0$, resulting in $\varepsilon_{\text {trk }}=96.3 \pm 0.3 \%$.

\subsection{Radiation Losses}

Electrons in our energy range of interest will lose energy mainly through Bremsstrahlung. A small fraction of the radiated photons could convert into a electron positron pair, $\gamma \rightarrow e^{+} e^{-}$. However, the radiated photon's energy is usually deposited in the calorimeter towers surrounding our signal tower. Due to the exclusive filter those electron pair events would be rejected if the deposited energy is above the noise level in those neighboring towers. The exclusive filter efficiency does not include those losses. We could measure the fraction of events that passed our exclusive cuts by using exclusive $e^{+} e^{-}$events from a Monte Carlo simulation using the LPAIR program, tracked through the full CDF detector simulation and reconstruction software. Knowing the number of exclusive Monte Carlo events that were reconstructed as electron pair events we can count the number of those that passed our exclusive filter.

$$
\varepsilon_{\text {no radiation }}=\frac{\# \text { Events }_{\text {exc e }^{+} \mathrm{e}^{-}}^{\text {pass }}}{\# \text { Events }_{\text {exc e }^{+} \mathrm{e}^{-}}}
$$


We call it the probability of no radiation or the acceptances for true electrons pairs passing the exclusive cuts. Values for different cuts on $\mathrm{E}_{\mathrm{T}}$ can be found in Table 6.14. The systematic uncertainty was estimated by varying the exclusive cuts by $\pm 10 \%$.

TABLE 6.14. Probabilities of no radiation for electron-positron pairs taking into account radiative losses via Bremsstrahlung. The energy fraction may be deposited into neighboring calorimeter towers and thus destroying the exclusiveness of the events. The full CDF simulation and reconstruction software was used in connection with the LPAIR Monte Carlo event generator for creating the exclusive electron pairs.

\begin{tabular}{lccc}
\hline$E_{\mathrm{T}}$ cut $(\mathrm{GeV})$ & 2.0 & 2.5 & 5.0 \\
\hline$\varepsilon_{\text {no radiation }}$ & 0.42 & 0.42 & 0.37 \\
Stat Err & \pm 0.001 & \pm 0.001 & \pm 0.002 \\
Syst Err & \pm 0.077 & \pm 0.078 & \pm 0.078 \\
\hline
\end{tabular}

\subsection{Final Exclusive Electron-Positron Pair Sample}

We were now left with our selected exclusive candidate sample of $e^{+} e^{-}$events (control study), extracted from the data of the run periods 8 to 13 where the specialized DIFF_DIPHOTON2 trigger was installed. The statistics of the final $e^{+} e^{-}$samples are shown in Table 6.13, separated by data taking period. We list numbers for three different $E_{T}$ cuts, namely 2.0, 2.5 and $5.0 \mathrm{GeV}$. For the final result only the candidate sample for the $E_{T}$ cut of $2.5 \mathrm{GeV}$ was used. All the following plots were made for the same candidate sample with $E_{T}>2.5 \mathrm{GeV}$. In Figure 6.25 the instantaneous bunch luminosities for the electron-positron pair candidates are shown. As expected, all our candidates were found between instant bunch luminosities ${ }^{7}$ of 0.4 and $2.5 \times 10^{30} \mathrm{~cm}^{-2} \mathrm{~s}^{-1}$, a region of no or very little pileup. Figure 6.26 shows the number of events over the effective luminosity per data taking period. The rate of exclusive $e^{+} e^{-}$events was constant for the different run periods.

Next we compare the kinematical variables of our exclusive event sample with an exclusive Monte Carlo sample generated with LPAIR program. The Monte Carlo sample was processed through the full CDF simulation and reconstruction software. The Monte Carlo sample had to pass all the cuts that had been also applied to the candidate data sample. Further, the Monte Carlo sample was normalized to the expected cross section taking into account all determined efficiencies as well as the available integrated luminosity. No background was subtracted in that comparison.

\footnotetext{
${ }^{7}$ Note, that the corresponding all-bunch instantaneous luminosity is 36 times higher, i.e. between 14 and $90 \times 10^{30} \mathrm{~cm}^{-2} \mathrm{~s}^{-1}$.
} 


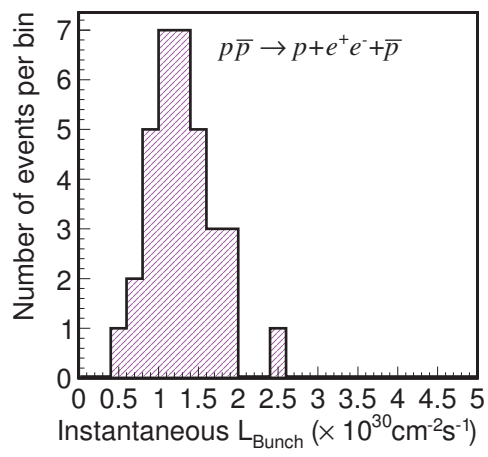

Figure 6.25. Instantaneous bunch luminosity of the exclusive $e^{+} e^{-}$ candidate events.

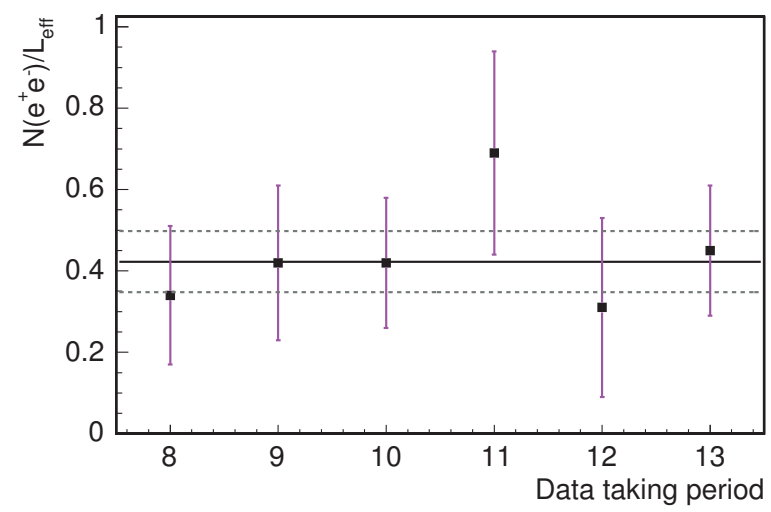

Figure 6.26. Number of $e^{+} e^{-}$events per effective luminosity. An $E_{\mathrm{T}}$ cut of $2.5 \mathrm{GeV}$ has been applied.

Figure 6.27 shows the single electron ${ }^{8} E_{T}$ on the left and the electron $E_{T}$ of the leading versus the second on the right. Figure 6.28 shows the pseudorapidity $\eta$ on the left and the azimuthal angle $\phi$ on the right for all electrons. The kinematical variables of the centrally produced system such as the longitudinal and the transverse momenta, the separation in $|\pi-\Delta \phi|$ and the 3D opening angle distribution can be seen in Figures 6.29 and 6.30. The quality of the tracks in form of the momentum $p$ over the calorimeter energy, $p / E$ for all electrons, is shown in Figure 6.31 on the left. On the right in Figure $6.31 \mathrm{p} / \mathrm{E}$ for the leading electron versus the second electron is

\footnotetext{
${ }^{8}$ When the electron and positron are indistinguishable we use the term electron only.
} 

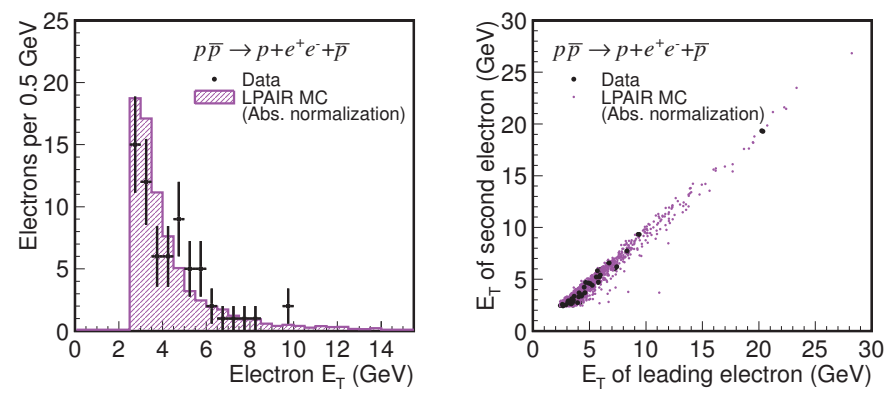

Figure 6.27. Kinematical comparison of $e^{+} e^{-}$candidates with LPAIR Monte Carlo events. $E_{T}$ distribution of all electrons (left). $E_{T}$ distribution of the leading electron versus the second electron (right).
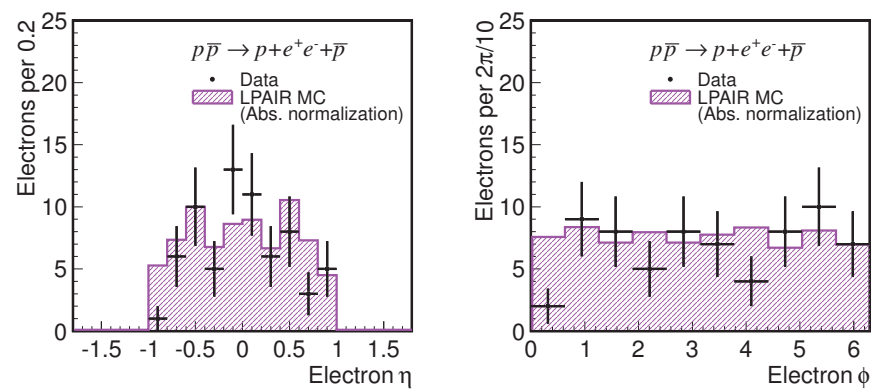

Figure 6.28. Kinematical comparison of $e^{+} e^{-}$candidates with LPAIR Monte Carlo events. $\eta$ distribution of all electrons (left) and the $\phi$ distribution (right).
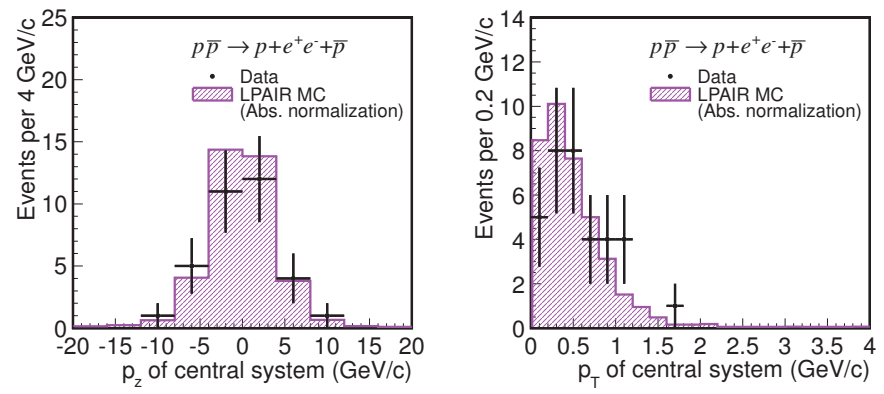

FiguRE 6.29. Kinematical comparison of $e^{+} e^{-}$candidates with LPAIR Monte Carlo events. The longitudinal momentum $p_{z}$ distribution of all electrons (left) and the transverse momentum $p_{t}$ distribution of the electron tracks (right). 
shown. Finally, the invariant mass of the centrally produced electron-positron pair system is shown in Figure 6.32 ${ }^{9}$. The comparison shows good agreement between data and Monte Carlo simulated events in all kinematical variables both in shape and magnitude. Applied Kolmogorov-Smirnov tests of the Monte Carlo sample versus data show no signs of disagreement. All the candidate show back-to-back electron-positron pairs with well balanced transverse energies $E_{T}$, i.e. the pairs lay close to a diagonal for the leading versus the second particle $E_{T}$ (see Figure 6.27 on the right). The momenta measured from the tracks is consistent with the energies of the calorimeter showers (see Figure 6.31). The 3D-opening angle plot of the electron-positron pair candidates shows no sign of any cosmic background. All in all these results establish confidence for the search for exclusive $\gamma \gamma$ production, as the selection criteria are very similar except for the tracking issue. As we see later when we present the calculation of the experimental cross sections of our observed processes, the $e^{+} e^{-}$cross section is in excellent agreement with the earlier CDF result [10].

\footnotetext{
${ }^{9}$ Note that there is no significant $Y \rightarrow e^{+} e^{-}$signal in the mass spectrum. The expectation for the $\Upsilon(1 S, 9460)$ is $\sim 1.7$ events.
} 

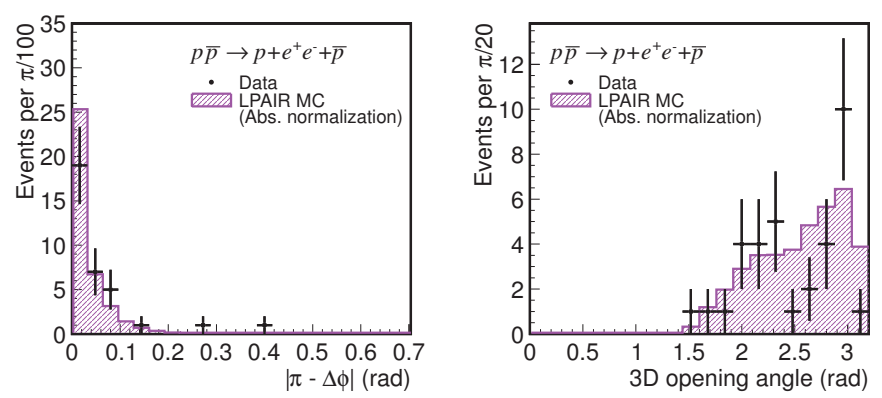

FiguRE 6.30. Kinematical comparison of $e^{+} e^{-}$candidates with LPAIR Monte Carlo events. The $|\pi-\Delta \phi|$ distribution of all $e^{+} e^{-}$pairs (left) and the $3 \mathrm{~d}$-opening-angle distribution (right).
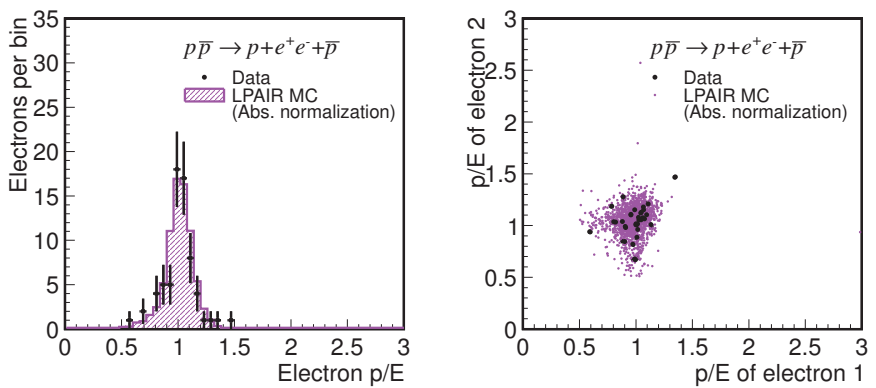

Figure 6.31. Kinematical comparison of $e^{+} e^{-}$candidates with LPAIR Monte Carlo events. The momentum over energy $p_{\text {track }} / E_{\text {cal }}$ distribution of all electrons (left) and the $p_{\text {track }} / E_{\text {cal }}$ distribution of the leading electron versus the second electron (right).
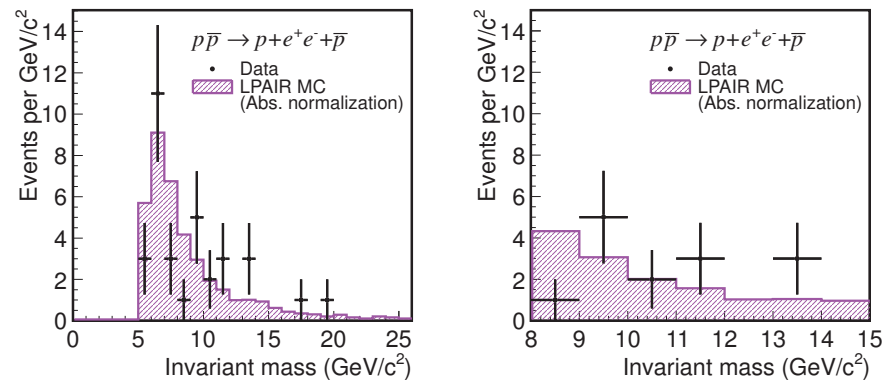

FigurE 6.32. Kinematical comparison of $e^{+} e^{-}$candidates with LPAIR Monte Carlo events. The reconstructed invariant mass of the $e^{+} e^{-}$pairs (left) and a magnification (right). 
TABLE 6.15. Number of exclusive $\gamma \gamma$ pairs split into different data taking periods.

\begin{tabular}{c|ccccc}
\hline \multirow{2}{*}{ Period } & \multicolumn{2}{|c}{$E_{\mathrm{T}}>2 \mathrm{GeV}$} & \multicolumn{2}{c}{$E_{\mathrm{T}}>2.5 \mathrm{GeV}$} & $E_{\mathrm{T}}>5 \mathrm{GeV}$ \\
\cline { 2 - 6 } & $\gamma \gamma$ & $\div \mathcal{L}_{\text {eff }}[\mathrm{pb}]$ & $\gamma \gamma$ & $\div \mathcal{L}_{\text {eff }}[\mathrm{pb}]$ & $\gamma \gamma$ \\
\hline 8 & 18 & 1.57 & 7 & 0.61 & 0 \\
9 & 15 & 1.29 & 9 & 0.78 & 0 \\
10 & 21 & 1.30 & 11 & 0.68 & 0 \\
11 & 12 & 1.05 & 5 & 0.44 & 0 \\
12 & 7 & 1.11 & 3 & 0.48 & 0 \\
13 & 18 & 1.04 & 8 & 0.46 & 2 \\
\hline Tot: & 91 & 1.22 & 43 & 0.58 & 2 \\
\hline
\end{tabular}

\section{Exclusive Diphotons}

\subsection{Selection of Candidates}

The final candidates for the exclusive photon pair study were selected from the sample of exclusive events as described in Section 1 of this Chapter. The only significant difference between photons and electrons are charged particle tracks in the tracking detectors. Therefore we required strictly no tracks in the events, in particular no tracks associated with the two reconstructed electromagnetic objects. This gave us a sample of good exclusive photon-pair candidates, possibly contaminated with a currently unknown number of exclusive $\pi^{0} \pi^{0}$ events or other background. The statistics of the final candidate sample are shown in Table 6.15, listed also for the single data-taking periods. We list events numbers for three different $E_{T}$ cuts, namely 2.0, 2.5 and $5.0 \mathrm{GeV}$, similar to the electron-pair "control study", but used the sample selected by the $2.5 \mathrm{GeV}$ cut for our final result. However, even in $\gamma \gamma$ events, tracks can occur due to conversion or Compton scattering which have to be discussed.

\subsection{Conversion Losses}

We need to take into account conversion and Compton processes in order to estimate the true amount of photon-pairs. A $\gamma \gamma$ event that includes such a process would be either rejected by the exclusive filter if the converted electron-positron pair deposits energy outside the signal towers, or due to the conversion tracks would not pass the "no track" selection rules. These losses we could measure in a similar way as the losses of electron-pairs due to Bremsstrahlung. We used our signal Monte Carlo simulated events produced by SUPERCHIC program in connection with a full CDF detector simulation and reconstruction. From the starting point of the number of 
TABle 6.16. Probability of no conversion for photon-pairs taking into account conversion and Compton scattering effects that may deposit energy into neighboring calorimeter towers, thus destroying the exclusiveness of the events, and not passing the "no track" cut. The full CDF simulation and reconstruction software was used in connection with the SUPERCHIC Monte Carlo event generator for creating the exclusive photon pairs.

\begin{tabular}{lccc}
\hline$E_{\mathrm{T}}$ cut $(\mathrm{GeV})$ & 2.0 & 2.5 & 5.0 \\
\hline$\varepsilon_{\text {no conversion }}$ & 0.583 & 0.568 & 0.482 \\
Stat Err & negligible & \pm 0.001 & \pm 0.003 \\
Syst Err & \pm 0.066 & \pm 0.063 & \pm 0.072 \\
\hline
\end{tabular}

reconstructed photon-pair events within $|\eta|<1.0$ that also passed our $E_{T}$ cut, we counted how many events pass our exclusive filter and the "no track" cut. Dividing this number by the total amount of probe events we received (and called it) the probability of no conversion, or the acceptance of $\gamma \gamma$ taking into account losses by those processes.

$$
\varepsilon_{\text {no conversion }}=\frac{\# \text { Events }_{\operatorname{exc} \gamma \gamma}^{\text {pass }}}{\# \text { Events }_{\operatorname{exc} \gamma \gamma}}
$$

The results are shown in Table 6.16 for the the different $E_{T}$ cuts used. However, as stated earlier, for the final result only the $E_{T}$ cut of $2.5 \mathrm{GeV}$ was used. The systematic uncertainty was estimated by varying the exclusive cuts $\pm 10 \%$, which are listed in Table 6.8.

\subsection{Final Exclusive Diphoton Sample}

We were now left with our selected exclusive candidate sample of $\gamma \gamma$ events, extracted from the data of run periods 8 to 13 where the specialized DIFF_DIPHOTON2 trigger was installed. All the following kinematical comparison plots were made using this candidate sample.

In Figure 6.33 the instantaneous single bunch crossing luminosities for the exclusive photon-pair candidates are shown. All our candidates occur between 0.4 and $2.4 \times 10^{30} \mathrm{~cm}^{-2} \mathrm{~s}^{-1}$ which corresponds to instantaneous all bunch luminosities between 14 and $86 \times 10^{30} \mathrm{~cm}^{-2} \mathrm{~s}^{-1}$. The number of exclusive photon-pair events divided by the effective luminosity for the different data taking periods can be seen in Figure 6.34. The exclusive photon-pair production rate is approximately constant throughout the data-taking periods 8 to 13 .

Next we compare some kinematical variables of our exclusive event sample with the exclusive Monte Carlo simulated signal sample generated with the SUPERCHIC 


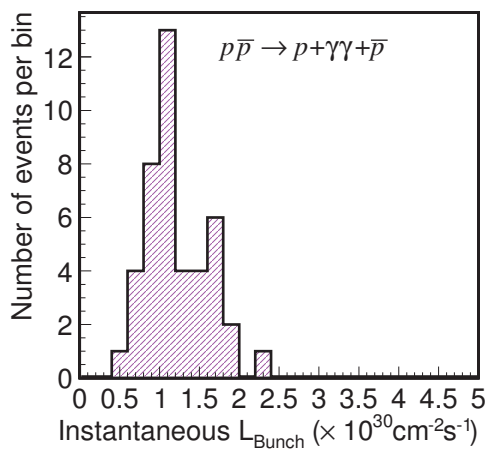

FiguRE 6.33. Instantaneous bunch luminosity of exclusive $\gamma \gamma$ candidate events.

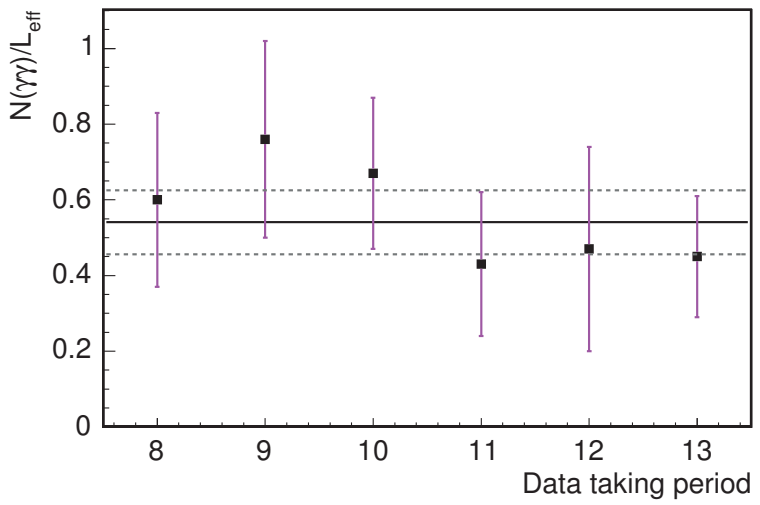

FiguRE 6.34. Number of photon-pair events per effective luminosity. An $E_{\mathrm{T}}$ cut of $2.5 \mathrm{GeV}$ was applied.

event generator program. The Monte Carlo sample had been further processed by the full CDF detector simulation and reconstruction software. The Monte Carlo sample had to pass all the cuts that had been also applied to the data candidate sample. For comparison we normalized the Monte Carlo simulated sample to the real data. A normalization to the expected cross section makes not much sense as the theoretical predictions have large uncertainties. In this case it is more valuable to compare the shapes of the kinematical distribution.

The transverse energy $E_{T}$ of all photons is shown in Figure 6.35 on the left and the comparison of the leading photon $E_{T}$ versus the second photon in the right. Figure 6.36 

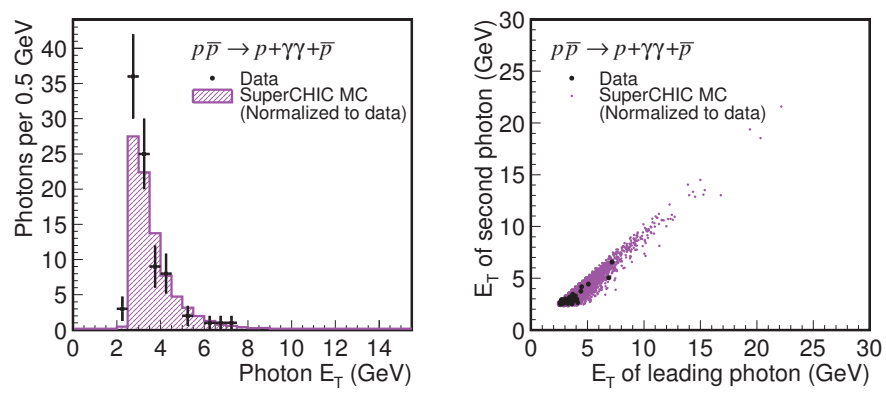

FIGURE 6.35. Kinematical comparison of $\gamma \gamma$ candidates with SUPERCHIC Monte Carlo events. The $E_{T}$ distribution of the photons (left) and the $E_{T}$ of the leading photon versus the $E_{T}$ of the other photon (right).
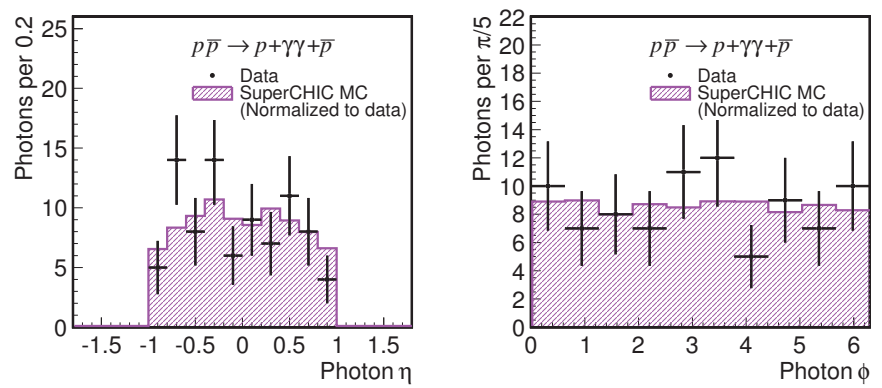

FiguRE 6.36. Kinematical comparison of $\gamma \gamma$ candidates with SUPERCHIC Monte Carlo events. The $\eta$ distribution of the photons (left) and the azimuthal $\phi$ distribution of the photons (right).
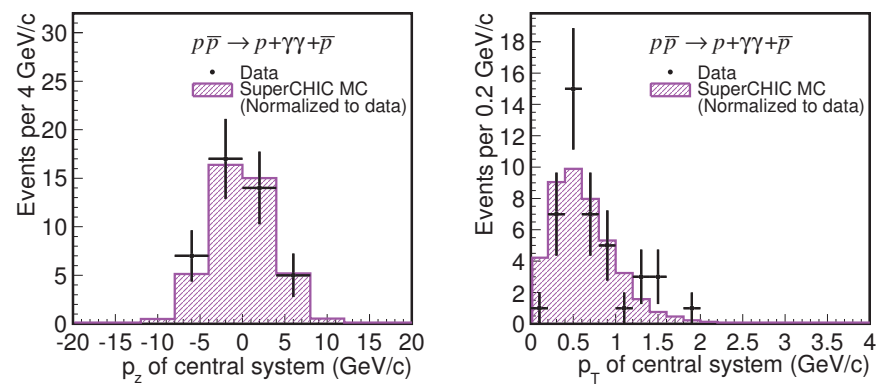

FiguRE 6.37. Kinematical comparison of $\gamma \gamma$ candidates with SUPERCHIC Monte Carlo events. The $p_{z}$ distribution of the photon pairs (left) and the $p_{T}$ distribution of the photon pairs (right). 

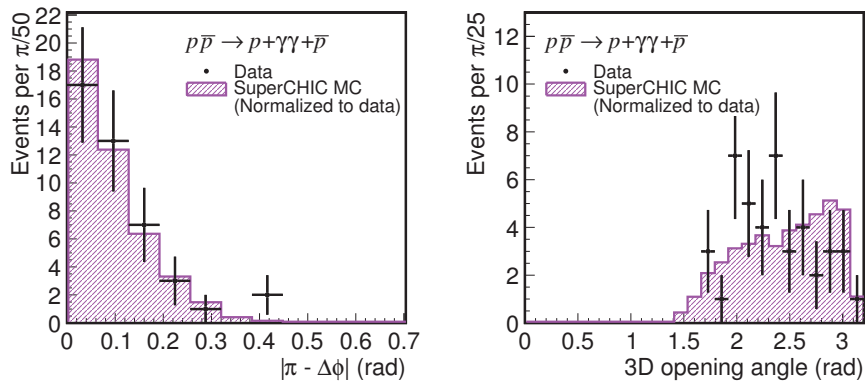

FIGURE 6.38. Kinematical comparison of $\gamma \gamma$ candidates with SUPERCHIC Monte Carlo events. The $|\pi-\Delta \phi|$ distribution of the photon pairs (left) and the 3D opening angle distribution of the photon pairs (right).
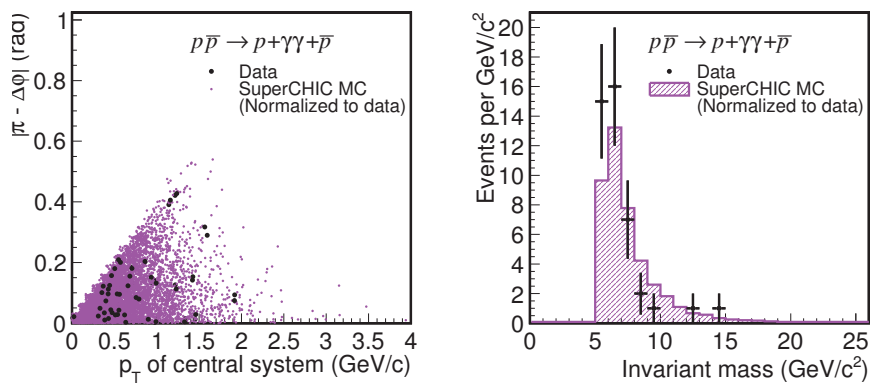

FIGURE 6.39. Kinematical comparison of $\gamma \gamma$ candidates with SUPERCHIC Monte Carlo events. The $p_{T}$ distribution of the photon pairs versus the $|\pi-\Delta \phi|$ distribution (left) and the reconstructed invariant mass distribution of the photon pairs (right).

shows the pseudorapidity $\eta$ of all photons on the left and their azimuthal angle $\phi$ on the right. The kinematical variables of the centrally produced system such as the longitudinal and transverse momenta $p_{z}, p_{t}$, the back-to-backness in form of $|\pi-\Delta \phi|$ as well as the 3D opening angle can be seen in Figures 6.37 and 6.38. Next in Figure 6.39 on the left we compare the transverse momentum of the central pair system with the azimuthal separation angle of the outgoing photons. Finally in Figure 6.39 on the right the invariant mass of the centrally produced photon-pair system is shown.

The comparison shows good agreement between the data and the simulated Monte Carlo sample in terms of distribution shapes. The distribution of the photon $E_{T}$ is as expected much steeper compared to electron-positron control sample which shows up 
as well in the event statistics for different minimum $E_{T}$ selections. All candidates show back-to-back photons. There are two candidates slightly over the expectation around a $|\pi-\Delta \phi|$ of 0.4 . Comparing those with transverse momentum $p_{T}$ of the pair system as done in Figure 6.39 on the left does not raise any concern. The transverse energies of the leading and the second photons are equally balanced and lay close to the diagonal (see Figure 6.35 on the left). To summarize, the comparison of the shapes between the data and the simulated events show good agreement. Kolmogorov-Smirnov tests had been applied for a comprehensive comparison. The quantitative comparison shows a slight difference as we see later in the experimental cross section calculation, but is in good agreement with the theoretical expectations. The theoretical uncertainty factor is not very well known, but is expected to be a factor $\underset{\div 2}{\sim 2}$ (at the time of this measurement).

\section{Backgrounds}

\subsection{Neutral Pion Background}

The $\pi^{0} \pi^{0}$ background to $\gamma \gamma$ is not a priori expected to be large in our mass region $M \gtrsim 5 \mathrm{GeV} / \mathrm{c}^{2}$. It has never been measured; the closest approximation is central exclusive $\pi^{+} \pi^{-}$in $p p$ collisions at the ISR at $\sqrt{s}=63 \mathrm{GeV}$ [100]. The cross section falls steeply with $M(\pi \pi) \sim e^{-2.8 M}$ (purely empirical, with $M$ in $\mathrm{GeV} / \mathrm{c}^{2}$ ) and there were no events above $\sim 3.5 \mathrm{GeV} / \mathrm{c}^{2}$. Note that the cross section for $p+p \rightarrow p+\pi \pi+p$ with both pions in a fixed central region decreases with increasing $\sqrt{s}[\mathbf{4}]$. Theoretically the only prediction for exclusive $\pi \pi$ in our region is from the Durham group (at our request) [2]. We quote: "At first sight it would appear that the cross section for this purely QCD process may be much larger than the $\gamma \gamma$ cross section and so would constitute an appreciable background, but fortunately this is not the case." Unlike the photon, the $\pi^{0}$ has a form factor of $f_{\pi} \sim 132 \mathrm{MeV}[\mathbf{1 3 2}]$ and the hard subprocess is suppressed by a factor $\left(f \pi / E_{\perp}\right)^{4}$. In addition, with forward (small $|t|$ ) outgoing protons, the $J_{z}=0$ selection rule suppresses exclusive $\pi \pi$ production. They conclude: "Therefore we can safely conclude that exclusive $\pi^{0}$ pair production will not constitute a large background to the central exclusive $p p \rightarrow p+\gamma \gamma+p$ process, even before any consideration of the efficiency with which $\pi^{0} \rightarrow \gamma \gamma$ mimics single $\gamma$ production."

At the time of the analysis the $\eta \eta$ production was estimated to be suppressed approximately by a factor of $(m(\pi) / m(\eta))^{2}=0.06$. However it is more complicated; it depends on the $\pi^{0}$ and $\eta$ wave functions, and the exchange in the $t$-channel. Back then we had consulted Khoze and Ryskin of the Durham group about this and recently they have confirmed that a $\eta \eta$ contribution to the $4 \gamma$ state compared to $\pi^{0} \pi^{0}$ should 
be very small [6]. For this reason we concentrate on discussing the exclusive $\pi^{0} \pi^{0}$ background.

In any case, $\pi^{0} \pi^{0}$ or $\eta \eta$ events can only constitute a background if one photon from each decay is not detected. Note that exclusive $p+p \rightarrow p+\gamma \pi^{0}+p$ events are forbidden (by C-parity), therefore we can classify events with two EM showers as either $\gamma \gamma$ or $\pi^{0} \pi^{0}(\eta \eta)$.

There is no standard CDF algorithm to distinguish between $\gamma$ and $\pi^{0}$ at these low energies in the CES. The $\pi^{0}$ decays promptly $\pi^{0} \rightarrow \gamma \gamma$ (see Appendix A4). The two photons have a minimum opening angle $\theta_{\gamma \gamma \text {, min }}=2 \arctan \left(m_{\pi} / p_{\pi}\right)$, which is when the decay is transverse to the direction of flight of the $\pi^{0}$. This is the most probable decay because phase space goes like $\sin \theta^{*} d \theta^{*}$. Thus the decay is flat in $\cos \left(\theta^{*}\right): \frac{d N}{d \cos \left(\theta^{*}\right)}=1 / 2$. The photon energy spectrum is flat within the limiting values $\left(E_{\pi} \pm p_{\pi}\right) / 2$. The probability that a photon hits a detector of a fixed solid angle is the same as the probability that its parent $\pi^{0}$ would have hit the detector had the $\pi^{0}$ not decayed. The minimum opening angle $\Delta \theta_{\min }$ between the two photons from $\pi^{0}$ decay $^{10}$ is $2 \tan ^{-1}(m(\pi) / p(\pi))=3.1^{\circ}$ for $p(\pi)=5 \mathrm{GeV} / c$, well separated in the CES chambers, which have a granularity $<0.5^{\circ}$. The minimum distance between the two photon showers, for a $5 \mathrm{GeV} \pi^{0}$, is then $L \tan \theta_{\gamma \gamma}(\min )=9.9 \mathrm{~cm}($ at $\eta=0)$ to 13.3 $\mathrm{cm}($ at $|\eta|=1.1)$. As it scales like $1 / p\left(\pi^{0}\right)$ the minimum separation is $>20 \mathrm{~cm}$ for $p\left(\pi^{0}\right)=2.5 \mathrm{GeV} / \mathrm{c}$, the lowest momentum considered in this new study. So it is not possible for the two photons from a $\lesssim 8 \mathrm{GeV} \pi^{0}$ to merge and appear as one shower. When the $\pi^{0}$-frame decay angle $\theta^{*}$ decreases with respect to the direction of flight, the rate decreases because of phase space, the angle $\theta_{\gamma \gamma}$ increases (so merging continues to be impossible) and the energy asymmetry $\left(E_{1}-E_{2}\right) /\left(E_{1}+E_{2}\right)$ grows.

The other way a $\pi^{0}$ can fake a direct photon is if one of the decay photons showers early and ranges out before the CES chambers (at $5.9 X_{0}$, including the coil), falls into an inactive region of the detector $(8 \%)$, or leaves a small signal consistent with noise. In Figure 6.40 the measured probability of a photon not having an interaction in material of $6 X_{0}$ radiation length is shown [133]. Photons with $E>1.5 \mathrm{GeV}$ have a $98 \%$ probability of making a shower before reaching the CES detector. Only $2 \%$ is the probability of the shower ranging out or punching through the material without being detected. Photons can be well simulated using, for example, the EGs Monte Carlo program [134], which is embedded in GEANT. The EM shower modeling in EGS is well very established compared to hadron-induced shower modeling.

Although this has not been well simulated in CDF for such low energy showers, it can be obtained from the $e^{+} e^{-}$data, which covers the same $E_{T}$ and $\eta$ regions.

\footnotetext{
${ }^{10} \mathrm{In}$ case of $\eta$ with $p(\eta)=5 \mathrm{GeV} / c$ the minimum opening angle between the two photons is $12.5^{\circ}$.
} 


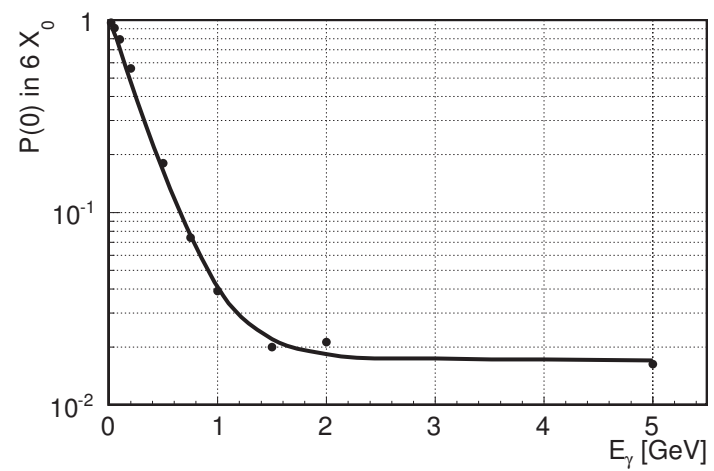

Figure 6.40. Probability of a photon not making a shower in lead at $6 X_{0}$ radiation lengths. Data measured by Sergey Striganov [133].

The strip chambers were not included in the DIFFDIPHOTON2 trigger, so we can count the fraction of EM showers from electrons/positrons (as a function of $E_{T}$ and $\eta$ ) that do not give a shower in the CES. We find that all of the 68 electrons (see Table 6.13) had a shower in the CES, so the range-out probability can be at most a few $\%$. The main difference between electron-initiated and photon-initiated showers is that electrons tend to shower a little earlier, so the fraction of electrons with no shower will be an upper limit on the fraction of photons (from $\pi^{0}$ ) with no showers, faking a single $\gamma$. Other differences that arise before the calorimeter are Bremsstrahlung photons from $e^{ \pm}$, and $\delta$-rays (always negative) from both $\gamma$ and $e^{ \pm}$. Also, photons can convert, $\gamma \rightarrow e^{+} e^{-}$, mostly in the silicon detectors.

4.1.1. Estimate of the Neutral Pion Background. We did not find any $\pi^{0} \pi^{0}$ events with 4 photon showers in our data of $1.11 \mathrm{fb}^{-1}$ integrated luminosity.

In case the two photons from the $\pi^{0}$ decay would be distributed across different calorimeter towers the event would be rejected by the strict exclusivity requirement. If the two photons reach the same calorimeter tower they are reconstructed by default as one EM object at the reconstruction level, thus constitute a background (the standard CDF production software is not able to reconstruct the two photons as separate objects within one calorimeter tower). Fortunately, as discussed already above, the two photons are expected to be well separated within our energy range, and using the CES we were able to reconstruct the number of separate clusters. The custom made software scans the CES chambers for well separated clusters with clear wire and strip hits above the noise level. The wire and strip hits must coincide. 

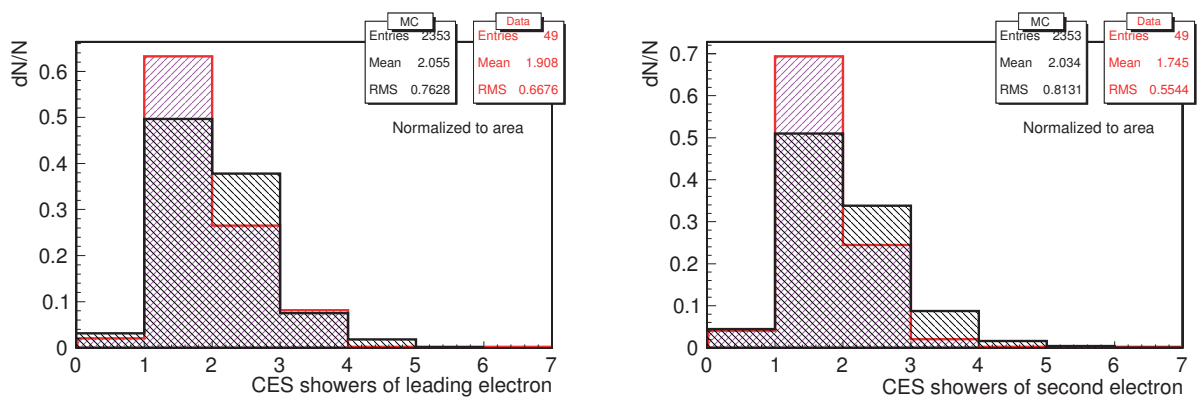

FigurE 6.41. Comparison of the reconstructed CES shower distribution of exclusive electron-positron pair data with the LPair Monte Carlo sample. The leading electron is shown on the left and the second electron on the right.

A study on statistical basis, using electron pair data and Monte Carlo methods, indicated that only a small fraction of our data consists of exclusive $\pi^{0} \pi^{0}$ background events. We compared the distribution of the reconstructed number of CES clusters of the $e^{+} e^{-}$events with the distribution obtained from the LPair Monte Carlo electronpositron pair sample. The comparison is shown in Figure 6.41. The obtained scale factor is utilized to tune the distributions of the number of reconstructed CES showers of the photon and neutral pion Monte Carlo samples. The latter had been produced by shooting $\pi^{0}$ 's randomly in azimuthal angle and in pseudorapidity from $-1<\eta<+1$ using the particle gun feature of the CDF simulation framework. We found that the number of reconstructible CES showers depend on the $E_{T}$ of the neutral pion. This dependency is shown in Figure 6.42 on the left in form of the mean number of reconstructed CES showers over $E_{T}$. In case of the photon sample we found no $E_{T}$ dependency. During the time of the analysis we had no information about the correct slope in $E_{T}$ of the exclusive neutral pions, but concluded that a flat $E_{T}$ distribution would not be too realistic. Consequently we produced $\pi^{0}$ Monte Carlo samples for fixed $E_{T}$ windows of $1 \mathrm{GeV}$ from 2 to $8 \mathrm{GeV}$. We constructed the distribution of the number of CES showers by composition of distributions from the different fixed $E_{T}$ windows (the $E_{T}$ range matched the range of our candidate sample). For the composition we used a $E_{T}$ slope similar to the one from the exclusive Monte Carlo photon sample as an approximation and convoluted it with the acceptance of a $\pi^{0}$ as a true single photon candidate (see Figure 6.42 on the right). This led to a more realistic estimate as we (to repeat) did not know the $E_{T}$ slope of the neutral pions. The systematic uncertainty 

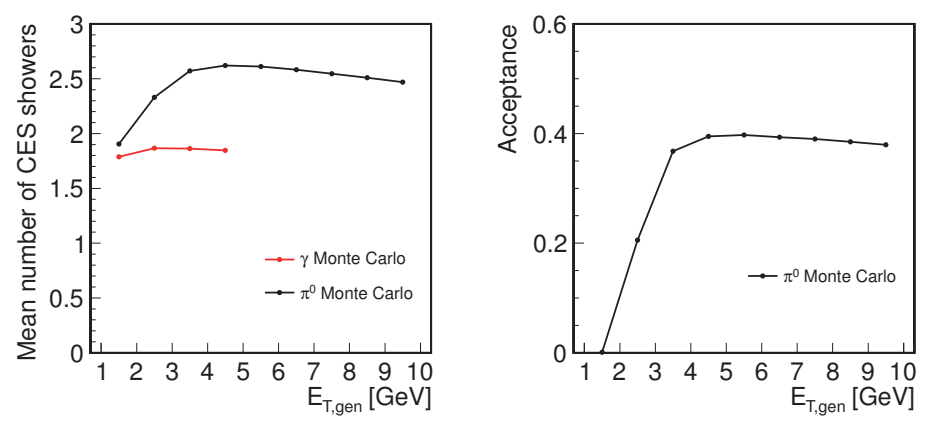

FiguRE 6.42. The mean number of reconstructed separate CES showers over $E_{T}$ for $\pi^{0}$ and photon Monte Carlo samples (left). The acceptance of $\pi^{0}$ 's as fake photons (right). The selection criteria are identical to the photon candidate selection. Note, we studied only the relevant $E_{T}$ range of the candidate sample.

was estimated by varying the slope (approximately exponential) by a factor of two, which has an effect on the shape of the reconstructed CES shower distribution.

Using Pearson's $\chi^{2}$ test we estimated the fraction of $\pi^{0}$ background in our candidate sample. The hypothesis is a combination of the scaled photon and $\pi^{0}$ Monte Carlo CES shower distributions,

$$
p d f_{\text {hypothesis }}^{\mathrm{CES}}=f p d f_{\pi^{0}}^{\mathrm{CES}}+(1-f) p d f_{\gamma}^{\mathrm{CES}}
$$

with $f$ being the fraction of the $\pi^{0}$ background. In Figures 6.43 to 6.45 we see the results for the leading EM object, the second EM object as well as the sum of both EM objects. Clearly we can see that the most likely fraction of $\pi^{0} \pi^{0}$ background is consistent with zero. With a confidence level of $95 \%$ we will have at most a fraction of $34 \%$ in our candidate sample. In our observed number of events we expect at $95 \%$ C.L. maximally 15 exclusive $\pi^{0} \pi^{0}$ background events.

As a sanity check on the quoted $\pi^{0} \pi^{0}$ fraction limit (0.34 at $95 \%$ C.L.) we ran a toy Monte Carlo 10, 000 times, generating distributions of the summed number of CES showers for 43 events. We found that $99.0 \%$ of these pseudo-experiments had a $\pi^{0} \pi^{0}$ fraction $<0.34$. While not a full simulation, this suggests that our quoted $95 \%$ C.L. is conservative.

\subsection{Dissociation Background}

Inelastic production of our processes of interest can be seen as falsely elastic if the fragments of a dissociated (anti)proton are completely unseen by the CDF detector 

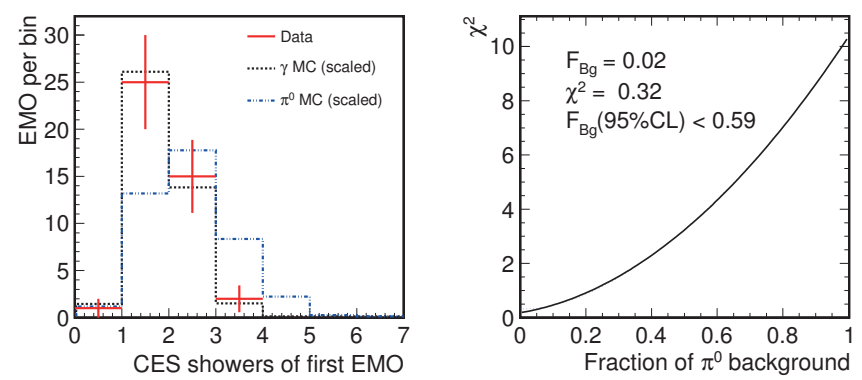

Figure 6.43. Comparison of the reconstructed CES shower distributions of the leading photon candidates with the photon and the $\pi^{0}$ Monte Carlo samples (left). The $\pi^{0}$ background fraction estimate using Pearson's $\chi^{2}$ test on the CES shower distributions (right).
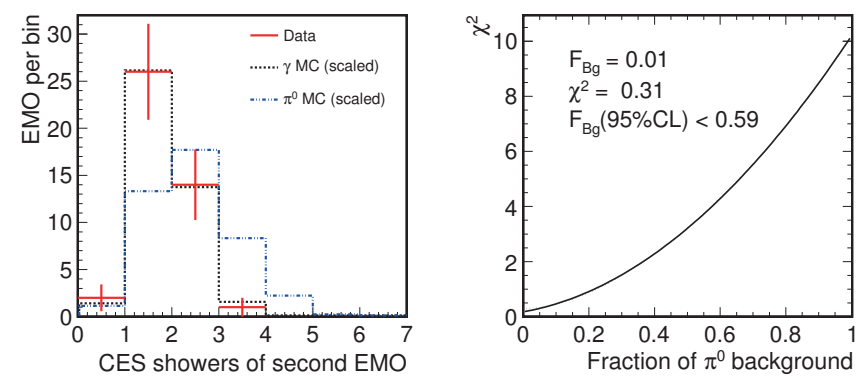

FIGURE 6.44. Comparison of the reconstructed CES shower distributions of the second photon candidates with the photon and the $\pi^{0}$ Monte Carlo samples (left). The $\pi^{0}$ background fraction estimate using Pearson's $\chi^{2}$ test on the CES shower distributions (right).
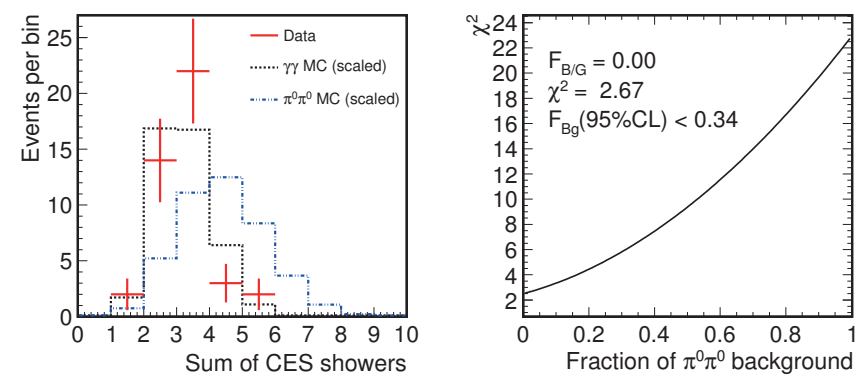

FiguRE 6.45. Comparison of the sum of the reconstructed CES shower distributions of the leading and second photon candidates with the photon and the $\pi^{0}$ Monte Carlo samples (left). The $\pi^{0}$ background fraction estimate using Pearson's $\chi^{2}$ test on the CES shower distributions (right). 


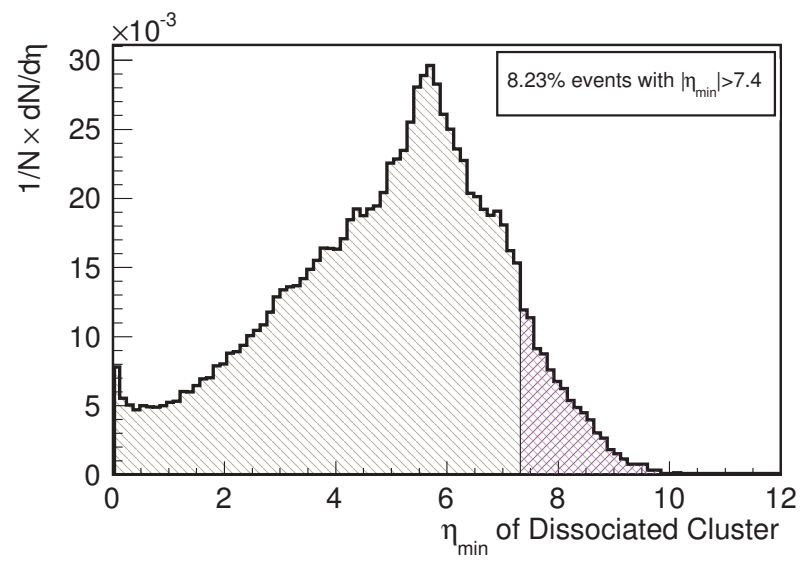

Figure 6.46. Minimum pseudorapidity of the dissociated proton clusters from inelastic production processes. The purple shaded region shows the fraction of dissociated protons unseen by the CDF detector. The CDF forward coverage ends with Beam Shower Counter BSC-3 at $\eta$ of 7.4. This particular example shows the unseen fraction for inelastic exclusive electron pair production with an $E_{T}$ cut of $2.5 \mathrm{GeV}$ for $|\eta|<1$. Here $8.2 \%$ of events with dissociated proton on one side will be unseen.

due to limited forward coverage. The most forward detector in use is the Beam Shower Counter BSC-3 whose coverage ends at $|\eta|$ of 7.4. For a quantitative estimate we use the same method as used in [10]. First the fraction of dissociated protons or antiprotons that are unseen by the CDF detector has to be found. This is done by counting the number of dissociated protons whose fragments have a minimum pseudorapidity of 7.4 that is the forward covarage limit of the BSC-3 compared to all protons generated. Because neither of the Monte Carlo generators for $\gamma \gamma$ and $e^{+} e^{-}$fragment the outgoing proton/antiprotons we needed to interface those with the MinBiasRockefeller Monte Carlo program MBR. This Monte Carlo fragments the protons and enables us to study their distribution. In Figure 6.46 the distribution of the minimum pseudorapidity of the proton/antiproton fragments is shown. The purple shaded area shows the fraction of protons whose fragments have a minimum pseudorapidity of 7.4, in other words, unseen by CDF. We obtain a fraction of 0.082 for $|\eta|>7.4$. For a probability of having an unseen inelastic proton dissociation, the efficiency of the BSC-3 stations for detecting proton fragments has to be known. As we required an upper limit for a rather small background contribution we took the result from the former CDF study [10]. We used the same cuts and the CDF detector configuration had not changed. The detector efficiency of the BSC-3 was 
TABLE 6.17. Exclusive $e^{+} e^{-}$cross sections obtained by LPAIR Monte Carlo simulation.

\begin{tabular}{cccc}
\hline \multicolumn{4}{c}{ Theoretical exclusive $e^{+} e^{-}$cross sections } \\
\hline $\mathrm{E}_{\mathrm{T}}$ cut $[\mathrm{GeV}]$ & Elastic $[\mathrm{pb}]$ & Single dissoc. $[\mathrm{pb}]$ & Double dissoc. $[\mathrm{pb}]$ \\
\hline 2.0 & $5.59 \pm 0.06$ & $3.70 \pm 0.03$ & $2.85 \pm 0.06$ \\
2.5 & $3.25 \pm 0.07$ & $2.28 \pm 0.02$ & $1.89 \pm 0.06$ \\
5.0 & $0.58 \pm 0.01$ & $0.50 \pm 0.01$ & $0.50 \pm 0.01$ \\
\hline
\end{tabular}

TABLE 6.18. Dissociation background of the exclusive electron pair production.

\begin{tabular}{ccc}
\hline & \multicolumn{2}{c}{ Dissociation background $\left(e^{+} e^{-}\right)$} \\
\hline $\mathrm{E}_{\mathrm{T}}$ cut $[\mathrm{GeV}]$ & Fraction & Background $($ events $)$ \\
\hline 2.0 & $0.12 \pm 0.01$ (stat) $\pm 0.03($ sys $)$ & $4.4 \pm 0.4($ stat $) \pm 0.9($ sys $)$ \\
2.5 & $0.12 \pm 0.01$ (stat) $\pm 0.03($ sys $)$ & $3.8 \pm 0.4($ stat $) \pm 0.9($ sys $)$ \\
5.0 & $0.13 \pm 0.02$ (stat) $\pm 0.03($ sys $)$ & $1.3 \pm 0.2($ stat $) \pm 0.3($ sys $)$ \\
\hline
\end{tabular}

$\varepsilon_{\mathrm{BSC}-3}=0.9 \pm 0.1$. This gave us the probability of an unseen proton dissociation of $P_{\text {upd }}=0.09 / \varepsilon_{\mathrm{BSC}-3}=0.10 \pm 0.01$ First we used the $e^{+} e^{-}$"Control Channel" using the LPair Monte Carlo for the inelastic cross section estimate. In Table 6.17 the cross section for elastic and inelastic production of exclusive $e^{+} e^{-}$pairs are listed. Now we computed the cross section for an unseen event with proton dissociation,

$$
\sigma_{\text {upd }}=2 P_{\text {upd }} \sigma_{\text {inel-el }}+p_{\text {upd }}^{2} \sigma_{\text {inel-inel }} \text {. }
$$

Knowing the total process cross section consisting of the elastic and the unseen inelastic part we can estimate the fraction of dissociation background within our candidate sample. In Table 6.18 the fractions of dissociation background for different $E_{T}$ cuts of our candidate selection are shown. The total uncertainty is dominated by the systematic uncertainty from the beam shower counters. The systematic uncertainty is estimated by varying the maximal pseudo-rapidity coverage in $\eta$ of the BSC-3 by \pm 0.2 . As can be seen in Figure 6.47 this changes the fraction of invisible proton dissociations.

In the case of exclusive photon pair production the situation looks a bit different. The present day existing Monte Carlo simulations do not calculate the kinematics for the outgoing (anti)protons. Therefore this study cannot be done for the exclusive photon pairs. However, as stated in the published paper we expect a smaller dissociation background compared to electron pair production [11]. In QCD-mediated processes there are fewer excitation states of the proton at lower masses. The Durham group emphasizes that "the forward proton dissociation is strongly suppressed, and the 


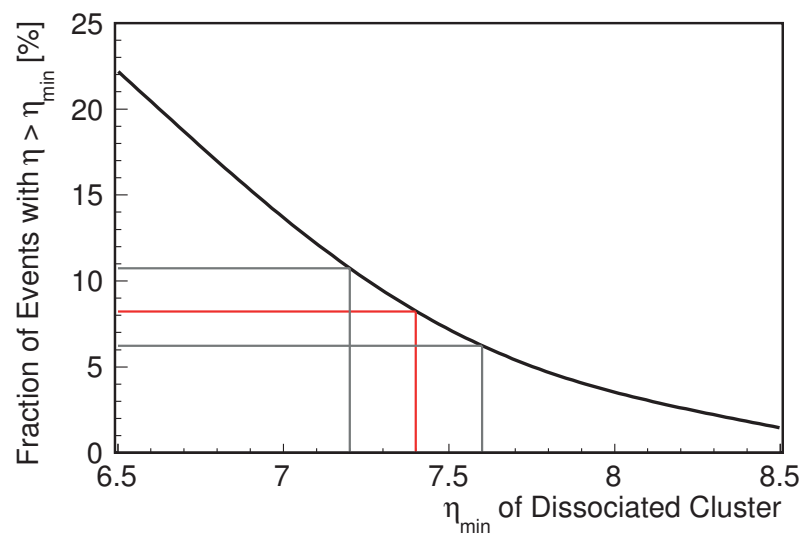

FiguRE 6.47. The fraction (\%) of events with $\eta$ of fragments larger than maximal $\eta$ coverage of the BSC-3 versus the minimum $\eta$ of the fragments. We vary $\eta_{\min }$ by \pm 0.2 to estimate the systematic uncertainty.

TABLE 6.19. Dissociation background estimate of the exclusive photon pair production.

\begin{tabular}{cc}
\hline \multicolumn{2}{c}{ Dissociation background $(\gamma \gamma)$} \\
\hline$E_{T}$ cut $[\mathrm{GeV}]$ & Background (events) \\
\hline 2.0 & $0.32 \pm 0.32($ sys $)$ \\
2.5 & $0.14 \pm 0.14$ (sys) \\
5.0 & $0.007 \pm 0.007$ (sys) \\
\hline
\end{tabular}

admixture of processes with incoming proton dissociation is not expected to exceed $0.1 \%$ [1] " for small masses of the centrally produced system. Durham took our selection rules into account using large rapidity gaps. We took the same approach as in [11] and took this upper limit to estimate the dissociation background for the photon pair study conservatively. The results are shown in Table 6.19.

\subsection{Other Background}

Other possible background for our exclusive processes could have been cosmic rays, and non-exclusive background (including neutral hadrons).

In the case of the $e^{+} e^{-}$study cosmic background has to be taken into account as cosmic rays could fake a back to back electron pair events when going through the vertex region. This would imply a large 3D opening angle $>3.05$ which looking at our 
data sample (Figure 6.30) does not exist. Cosmic rays are much more of an issue with $\mu^{+} \mu^{-}$events. We agree with the result of the previous CDF study [10] that estimated the cosmic background to be negligible which will not change by going to a lower $E_{T}$ threshold.

Non-exclusive backgrounds are inclusive events with some of the particles falling into a detector crack or ranging out before reaching the calorimeter and such faking a 2 EM object event with tracks or no tracks for the electron-pair study or the photon-pair study, respectively. In the electron case we could have events that would fake electron signatures such as narrow jets with a single track.

Neutral hadron background, such as $\gamma \gamma+$ long-lived $K_{L}^{0}, n, \bar{n}$ must be negligible. Their showers would normally be killed by the exclusivity cuts, and the $\gamma \gamma$ kinematics would not be consistent with the exclusive $\gamma \gamma$ process, as it is for the data.

\section{Results and Discussion}

Before discussing the results of the photon pair study we discuss our control channel, namely exclusive $e^{+} e^{-}$production in $p \bar{p}$ collisions. This was previously measured in CDF [10]. The purpose of revisiting was as a check on the quality of the new data and of the chosen cuts, and to give confidence in the analysis methods for the exclusive photon pair search. Except for the fact that electrons leave tracks in the tracking detectors, there is no difference between $e^{+} e^{-}$and $\gamma \gamma$ in this analysis.

\subsection{Cross Section of the Exclusive Electron-Positron Pair Production}

All the measured ingredients for the cross section measurement using Equation (98) are given in Table 6.20.

The total efficiency was calculated by:

$$
\varepsilon_{\mathrm{tot}}=\varepsilon_{\mathrm{trig}}^{2} \cdot \varepsilon_{\mathrm{rec}} \cdot \varepsilon_{\mathrm{id}}^{2} \cdot \varepsilon_{\mathrm{trk}}^{2} \cdot \varepsilon_{\mathrm{norad}} \cdot \varepsilon_{\mathrm{excl}}
$$

with $\varepsilon_{\text {rec }}$ and $\varepsilon_{\text {norad }}$ being on an event-by-event basis and $\varepsilon_{\text {trig }}, \varepsilon_{\text {id }}$ and $\varepsilon_{\text {trk }}$ are for single electrons. The theoretically expected cross section for elastic production of exclusive electron pairs was obtained by the LPAIR Monte Carlo event generator and can be found in Table 6.17. Those cross sections were calculated for $\sqrt{s}=1960 \mathrm{GeV}$ and restricted to the CDF central rapidity region of $-1<\eta<+1$. For an $E_{T}$ cut of $2.5 \mathrm{GeV}$ we expected a cross section of $\sigma_{\mathrm{LPAIR}}^{|\eta|<1, E_{T}>2.5 \mathrm{GeV}}=3.25 \pm 0.07 \mathrm{pb}$. For our signal sample of 34 candidates and a background estimate of 3.8 events we obtained a cross section of:

$$
\sigma_{e^{+} e^{-} \text {exclusive }}^{|\eta|<1, E_{T}>2.5 \mathrm{GeV}}=2.88_{-0.48}^{+0.57}(\text { stat }) \pm 0.63 \text { (syst) } \mathrm{pb} .
$$


TABLE 6.20. Summary of all relevant parameters for the measurement of the exclusive electron-pair cross section for a $E_{\mathrm{T}}$ cut of $2.5 \mathrm{GeV}$.

\begin{tabular}{lccc}
\hline & Value & Stat. error & Syst. error \\
\hline Integrated luminosity $\mathcal{L}_{\text {int }}$ & $1.11 \mathrm{fb}^{-1}$ & \multicolumn{2}{c}{ $\pm 0.7 \mathrm{pb}^{-1}$} \\
$e^{+} e^{-}$events & 34 & & \\
\hline Trigger efficiency & 0.920 & \pm 0.009 & \pm 0.018 \\
Reconstruction efficiency & 0.508 & \pm 0.007 & \pm 0.016 \\
Identification efficiency & 0.912 & \pm 0.017 & \pm 0.013 \\
Tracking efficiency & 0.963 & \multicolumn{2}{c}{0.003} \\
Probability of no radiation & 0.419 & \pm 0.001 & \pm 0.078 \\
Exclusive efficiency & 0.0680 & negligible & 0.004 \\
\hline Dissociation b/g (events) & 3.8 & 0.4 & 0.9 \\
\hline
\end{tabular}

TABLE 6.21. Comparison of theoretical expected and measured cross section (in $\mathrm{pb}$ ) for the exclusive $e+e^{-}$control channel.

\begin{tabular}{ll}
\hline & Exclusive Electron-Pair Production \\
\hline Theoretical & $\sigma_{\mathrm{LPAR}}^{|\eta|<1, E_{T}>2.5 \mathrm{GeV}}=3.25 \pm 0.07 \mathrm{pb}$ \\
Measured & $\sigma_{e^{+} e^{-} \text {exclusive }}^{|\eta|<1, E_{T}>2.5 \mathrm{GeV}}=2.88_{-0.48}^{+0.57}$ (stat) \pm 0.63 (syst) pb \\
\hline
\end{tabular}

The measured cross section is in good agreement with the theoretical (QED) cross section. Both measured and predicted cross sections are also shown in Table 6.21. With this successful result in the "control channel" we proceed to the photon pair measurement with confidence that indeed using the same methods in the search for CEP of diphotons we will have trustworthy results.

\subsection{Cross Section of the Exclusive Photon Pair Production}

Table 6.22 gives all the important factors for the measurement. The theoretical expectations calculated with the SUPERCHIC Monte Carlo event generator for 2 gluon parton density functions (MRST99, MSTW08LO) can be found in Table 6.23. We used the same equations as for the electron measurement, (98) and (117), replacing the acceptance for radiative electrons $\varepsilon_{\text {rad }}$ and the tracking efficiency $\varepsilon_{\text {trk }}$ with the efficiency for non converting photons $\varepsilon_{\text {no conv }}$ which is a per-event factor.

$$
\varepsilon_{\mathrm{tot}}=\varepsilon_{\mathrm{trig}}^{2} \cdot \varepsilon_{\mathrm{rec}} \cdot \varepsilon_{\mathrm{id}}^{2} \cdot \varepsilon_{\mathrm{no} \mathrm{conv}} \cdot \varepsilon_{\mathrm{excl}}
$$

With an $\mathrm{E}_{\mathrm{T} \text {, min }}$ of $2.5 \mathrm{GeV}$ and restricting to the central $\mathrm{CDF}$ detector region of $-1<\eta<1$ we measure a cross section of the elastic exclusive photon-pair production 
TABLE 6.22. Summary of all relevant parameters for the measurement of the exclusive photon-pair cross section for a $E_{\mathrm{T}}$ cut of $2.5 \mathrm{GeV}$.

\begin{tabular}{lccc}
\hline & Value & Stat. error & Syst. error \\
\hline Integrated luminosity $\mathcal{L}_{\text {int }}$ & $1.11 \mathrm{fb}^{-1}$ & & $\pm 0.7 \mathrm{pb}^{-1}$ \\
$\gamma \gamma$ events & 43 & & \\
\hline Trigger efficiency & 0.918 & \pm 0.005 & \pm 0.018 \\
Reconstruction efficiency & 0.553 & \pm 0.005 & \pm 0.029 \\
Identification efficiency & 0.927 & \pm 0.017 & \pm 0.013 \\
Exclusive efficiency & 0.0680 & negligible & 0.004 \\
Probability of no conversions & 0.568 & \pm 0.001 & \pm 0.063 \\
\hline$\pi^{0} \pi^{0}$ b/g (events) & 0 & & $<15$ (95\% C.L.) \\
Dissociation b/g (events) & 0.14 & & 0.14 \\
\hline
\end{tabular}

TABLE 6.23. Theoretical exclusive photon-pair production cross section predictions (in pb) for various cuts using the MRST99 and MSTW08LO partons. The cross sections have been calculated by the SUPERCHIC Monte Carlo generator [2]. Apart from the strong PDF dependence there are other uncertainties in the calculations.

\begin{tabular}{lccc}
\hline & $E_{\mathrm{T}}>2 \mathrm{GeV}$ & $E_{\mathrm{T}}>2.5 \mathrm{GeV}$ & $E_{\mathrm{T}}>5 \mathrm{GeV}$ \\
\hline MRST99 & 0.806 & 0.351 & 0.027 \\
MSTW08LO & 3.694 & 1.421 & 0.078 \\
\hline & $M_{\min }=4 \mathrm{GeV}$ & $M_{\min }=5 \mathrm{GeV}$ & $M_{\min }=10 \mathrm{GeV}$ \\
\hline MRST99 & 1.307 & 0.553 & 0.039 \\
MSTW08LO & 6.413 & 2.366 & 0.120 \\
\hline
\end{tabular}

of:

$$
\sigma_{\gamma \gamma \text { exclusive }}^{|\eta|<1, E_{T}>2.5 \mathrm{GeV}}=2.48_{-0.35}^{+0.40} \text { (stat) }{ }_{-0.51}^{+0.40} \text { (syst) } \mathrm{pb}
$$

This is in good agreement with the theoretical prediction by the Durham group (Table 6.23) taking into account that these predictions have uncertainty factors of 2 to 3 .

We can compute the significance of our measurement by asking for the probability that our background fluctuates up to the observed number of events. We took the estimated total background of $0.14_{-0.14}^{+5.6}$ events, that is the combination of the estimated exclusive $\pi^{0} \pi^{0}$ background and the inelastic $\gamma \gamma$ background. Using the prior-predictive method for Poisson distributed data we obtained a $p$-value of $3.9 \times 10^{-8}$. Expressed in Gaussian terms this corresponds to a probability of having a fluctuation greater than 
TABLE 6.24. Comparison of theoretical expected and the measured cross section (in $\mathrm{pb}$ ) for the exclusive photon-pair production.

\begin{tabular}{ll}
\hline Exclusive Photon-Pair Production \\
\hline Theoretical & $\sigma_{\text {SUPERCHIC }}^{|\eta|<1, E_{T}>2.5 \mathrm{GeV}}=0.35_{\div 2}^{\times 2} \mathrm{pb}($ MRST99) \\
& $\sigma_{\text {SUPERCHIC }}^{|\eta|<1, E_{T}>2.5 \mathrm{GeV}}=1.4_{\div 2}^{\times 2} \mathrm{pb}($ MSTW08LO $)$ \\
& $\sigma_{\text {SUPERCHIC }}^{|\eta|<1, E_{T}>2.5 \mathrm{GeV}}=2.2_{\div 2}^{\times 2} \mathrm{pb}($ CTEQ6L) \\
Measured & $\sigma_{\gamma \gamma \text { exclusive }}^{|\eta|<1, E_{T}>2.5 \mathrm{GeV}}=2.48_{-0.35}^{+0.40}$ (stat) ${ }_{-0.51}^{+0.40}$ (syst) pb \\
\hline
\end{tabular}

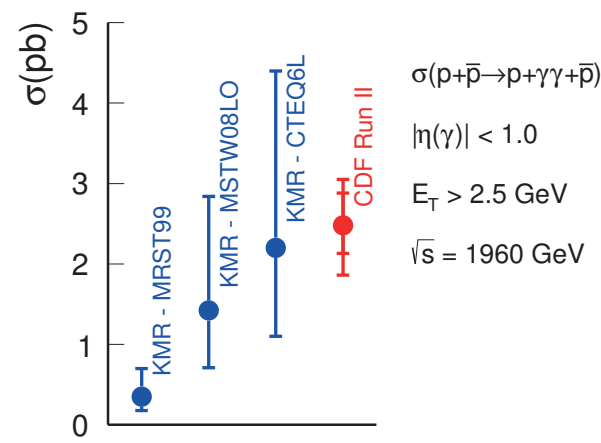

Figure 6.48. Comparison of the measured cross section with theoretical expected (in pb) for the exclusive photon-pair production. The theoretical expectations are computed for 3 different parton density functions (PDF).

$5.4 \sigma$ in the case of a normalized Gaussian distribution. We therefore claim the first observation of exclusive photon-pair production in hadron-hadron collisions with a significance greater than $5 \sigma$.

Comparing the result with the latest theoretical predictions made by the KRYSTHAL collaboration ${ }^{11}$ (summarized in Table 6.24 and Figure 6.48) we can put constraints on the theoretical model $[3]$. To remind the reader, the central exclusive process of producing a diphoton system, a strong interacting process, is seen as a standard candle for this class of reactions. It is a much cleaner process with less theoretical issues compared to the the CEP of $\chi_{c}$ mesons. The constraint of the measurement acts on the non-perturbative part and especially on the un-integrated gluon densities.

\footnotetext{
${ }^{11}$ Collaboration of V.A. Khoze, M.G. Ryskin and L.A. Harland-Lang.
} 
The non-perturbative part includes the additional soft diffractive processes between the incoming hadrons and between the incoming hadrons and the gluons of the central fusion process, described by $S_{\text {eik }}$ and $S_{\text {enh }}$ for the latter. Newer reconsiderations by the KRYSTHAL collaboration leave the soft survival factors for the Tevatron at the previous obtained values by considering our result. However, the authors claim that motivation can be found to lower these values by a factor of $\sim 2$ using latest LHC data $[\mathbf{1 3 5}]$.

Discussing the impact on the gluon densities, the authors of the latest predictions say that "we can in principle use these CEP measurements to shed some light on the gluon PDF in this low- $x$ and low- $Q^{2}$ region, where it is poorly determined". By comparison the agreement between the result and the prediction using MSTW08LO is cleary visible whereas from the result using the MRST99 gluon densities one can spot some discrepancies. Both are leading order, LO PDFs. The uncertainty on the cross section estimates is large as the calculations are proportional to the 4th power of the gluon-densities which themselves contain large uncertainties as said above. In fact higher order contributions in the DGLAP formalism are far from negligible at low- $x$. Next to leading order, NLO PDFs produce smaller predictions as the gluon densities are smaller for low- $x$. The differences between LO and NLO calculations are used as uncertainty factors for the theoretical predictions. 



\section{CHAPTER 7 \\ Conclusion and Outlook}

This thesis presents the first successful observation of the central exlusive production of diphotons at the Tevatron in proton-antiproton collisions of $\sqrt{s}=1.96 \mathrm{TeV}$ using the Run II Collider Detector at Fermilab. Moreover, we claim the first observation of such a process at a hadron collider with a significance of above $5 \sigma$ in Gaussian standards. Confidence was gained by simultaneously measuring the QED central exclusive production of electron-positron pairs using similar techniques. The measured cross section agrees well with our previous results and theoretical predictions.

The measured cross section of the CEP of $\gamma \gamma$ clearly constrains the theoretical models. Within the Durham approach this measurement puts extra constraints on the use of gluon density functions, one source of large uncertainty factors. It clearly favors the use of LO PDFs over NLO calculations.

As the exclusive $\gamma \gamma$ production process is closely related to the exclusive Higgs boson production $p p \rightarrow p+H+p$ at the LHC, it is often called a standard candle process. There was much uncertainty about the possibility of producing a Higgs boson with no hadrons at the LHC. The observation of exclusive diphotons in hadron-hadron collisions shows that exclusive Higgs boson production will happen in case its existence is confirmed, and certainly constrains the cross section.

Recently the CMS collaboration published results on their search for central exclusive diphoton production. They did not observe any candidates and set an upper limit on the production cross section $[\mathbf{1 3 6}]$. Without dedicated low luminosity runs in future to avoid pile-up it might be nearly impossible to find candidates at LHC with the current design of the experiments. In the case of being able to tag the outgoing protons with forward spectrometers it might of course change the situation in the future.

For the time being more details can be retrieved by refining the search for exclusive diphotons at the CDF experiment. In the present data there is still potential for finding more statistics by enlargening the pseudo-rapidity range and/or applying a new exclusive filter. More work is needed to study photon background separation power beyond the central pseudo-rapidity region from $1<|\eta|<\sim 1$.8. Tracking is much worse in this region meaning that electrons could be a substantial background 
for the photons. A newly developed exclusive filter shows already some statistics enhancement, however no final results are yet available.

Recently interest has also grown for measuring cross sections for the production of exclusive neutral meson pairs $\left(\pi^{0}, \eta, \eta^{\prime}\right)[6,137]$. A separate search for CEP of neutral pion pairs using the data recorded with the same trigger setup as used in the diphoton analysis did not reveal any candidates so far.

In the last year of the Tevatron running a new diffractive trigger was installed, requiring two central electromagnetic or hadronic showers with $E_{T}>0.5 \mathrm{GeV}$ and forward gaps using the beam shower counters and the plug calorimeter as a veto. CDF II was able to record 115 million events at $1.96 \mathrm{TeV}$ and 22 million events at $900 \mathrm{GeV}$.

The introduction of the new trigger with the lower threshold for electromagnetic showers in the central region of the CDF detector gives us the opportunity to search for light meson pairs. Using the same analysis framework as for the $\gamma \gamma$ study we will scan the central projective calorimeter for four distinct isolated photon candidates being the main decay products of the neutral mesons pairs, mainly $\pi^{0} \pi^{0}$ or $\eta \eta$. Allowing additionally two or four charged particle tracks, $\eta \eta^{\prime}$ or $\eta^{\prime} \eta^{\prime}$ pairs could be in reach. The $\eta^{\prime}$ meson decays mainly to $\pi^{+} \pi^{-}$plus an additional $\eta$ or $\gamma$. Theorists expect $\eta \eta>\pi^{0} \pi^{0}$ as the $\eta$ is an isoscalar with a larger gluon component. The cross sections for meson pair production including $\eta^{\prime}$ are even more enhanced. Measuring the production of light neutral mesons (i.e. $\eta, \eta^{\prime}$ ) constrains non-perturbative models and opens the door to understand their gluonic structure [6]. The production cross section of these mesons is highly sensitive to their not very well known gluonic content. 


\section{APPENDIX A}

\section{Kinematics}

\section{Mandelstam Variables}

It is useful to introduce some basic kinematics in connection to particle scattering processes. The focus is on a two-body scattering process, $1+2 \rightarrow 3+4$, as shown in Figure A.1 for example. The 4-momentum of each particle is written as $p_{i}=\left(E_{i}, \boldsymbol{p}_{i}\right)$ and $p^{2}=E^{2}-\boldsymbol{p}^{2}$. It is conveniant to use the Lorentz invariant Mandelstam variables

$$
\begin{aligned}
& s=\left(p_{1}+p_{2}\right)^{2} \\
& t=\left(p_{1}-p_{3}\right)^{2} \\
& u=\left(p_{1}-p_{4}\right)^{2} .
\end{aligned}
$$

The sum of the variables are equal to the sum of the particle masses squared,

$$
s+t+u=m_{1}^{2}+m_{2}^{2}+m_{3}^{2}+m_{4}^{2} .
$$

The diagram in Figure A.1 shows three different processes. The process $1+2 \rightarrow 3+4$ is called the $s$-channel process. The $t$-channel processes is found by turning the diagram about $90^{\circ}$ and reversing the kinematics and quantum numbers of particle 2 and 3 , resulting in $1+\overline{3} \rightarrow \overline{2}+4$. If the particles 2 and 4 of the s-channel process are exchanged, including momentum and quantum numbers, one gets the $u$-channel process $1+\overline{4} \rightarrow \overline{2}+3$. Reversing momentum and quantum numbers creates an anti-particle denoted by the bar.

It turns out that only two of the Mandelstam variables are independent. Usually one uses $s$ and $t$. After some arithmetics using energy and three-momenta in the

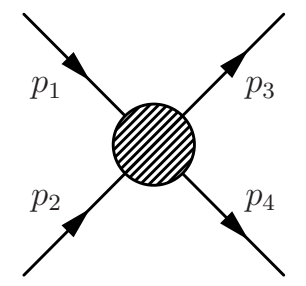

Figure A.1. Diagram for two-particle scattering, $1+2 \rightarrow 3+4$. 


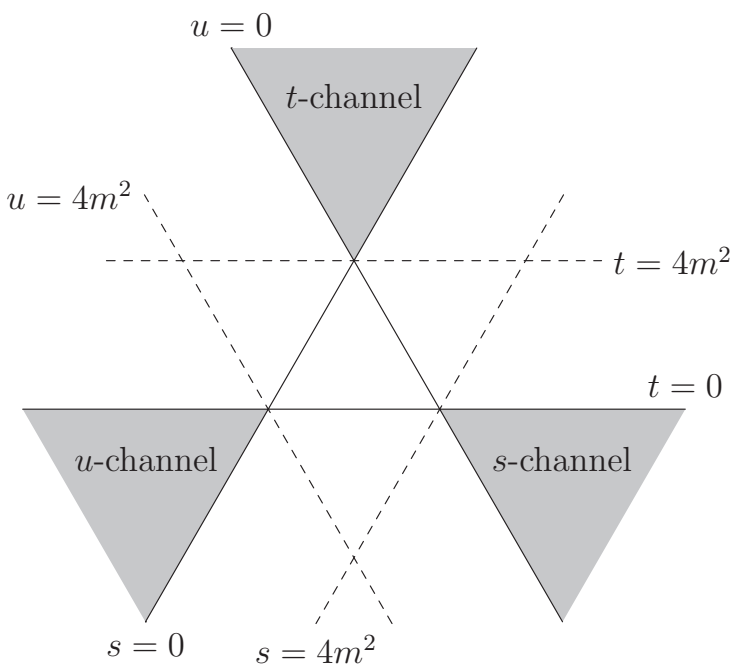

Figure A.2. Mandelstam presentation of the physical regions of the $\mathrm{s}-, \mathrm{t}-$ and $\mathrm{u}$-channels. Note: Here the masses of the scattered particles are assumed to be equal. For non-equal masses the idea is the same but the diagram would not be that symmetric.

center of mass frame and considering equal masses one finds the following boundaries of the physical allowed kinematical regions in the $s$-channel,

$$
\begin{aligned}
& s=4\left(\boldsymbol{p}^{2}+m^{2}\right) \\
& t=-2 \boldsymbol{p}^{2}(1-\cos \theta) \\
& u=-2 \boldsymbol{p}^{2}(1+\cos \theta) .
\end{aligned}
$$

In Figure A.2 the physical allowed kinematical regions of the two-body scattering process are shown. For non-equal masses the expressions especially for $t$ and $u$ are more complicated and so is the Mandelstam plot. The three kinematical regions are not overlapping. The scattering amplitudes, that are function of the Mandelstam variables can be analytically continued to between those different kinematical regions. The possibility to exchange the processes between the different kinematical regions as mentioned above, is known as crossing. 


\section{Diffractive Processes}

Here, some definitions are listed that are frequently used in diffractive low-x physics. Low-x, often named Feynman-x, is defined as

$$
x=\frac{p_{l}}{p}=1-\frac{M_{X}^{2}}{s},
$$

for an center of mass system in an environment with two head on colliding hadrons, where $p_{l}$ is the longitudinal momentum of the outgoing hadron in beam direction. Here $M_{X}^{2}=\left(p_{1}+p_{2}-p_{3}\right)^{2}$ is the squared mass of system $X$ as shown in Figure A.3. The fractional momentum loss $\xi$ is defined as $\xi=1-x$. Related to the longitudinal momentum is the rapidity $y$ that is defined

$$
y=\frac{1}{2} \ln \left(\frac{E+p_{l}}{E-p_{l}}\right) .
$$

In case one deals with approximately massless particles at high energies with $E=|\boldsymbol{p}|$ and using $p_{l}=|\boldsymbol{p}| \cos \theta$ one easily finds

$$
y_{\left.\right|_{m=0}} \approx-\ln \left[\tan \left(\frac{\theta}{2}\right)\right] \equiv \eta
$$

which is now called pseudorapidity $\eta$ where $\theta$ is the polar angle of the outgoing particle.

In case of a single diffraction with one hadron dissociating into a system $X$ and the other hadron surviving the collision one can estimate the rapidity gap as illustrated in Figure A.4. The maximum pseudorapidity value of the system $X$ is approximately

$$
\eta_{X, \max } \approx-\ln \frac{m \sqrt{s}}{M_{X}^{2}} .
$$

The pseudorapidity of the surviving hadron is

$$
\eta_{\text {surv }}=\ln \frac{\sqrt{s}}{m} .
$$

The difference will estimate the rapidity gap,

$$
\Delta \eta \approx \ln \frac{\sqrt{s}}{m}+\ln \frac{m \sqrt{s}}{M_{X}^{2}} \approx \ln \frac{s}{M_{X}^{2}}=-\ln (1-x),
$$

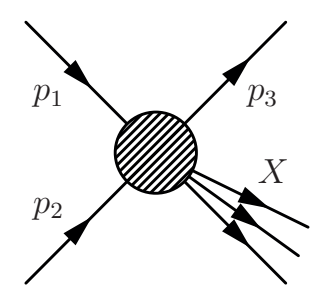

Figure A.3. Diagram for single diffractive dissociation, $1+2 \rightarrow 3+X$. 


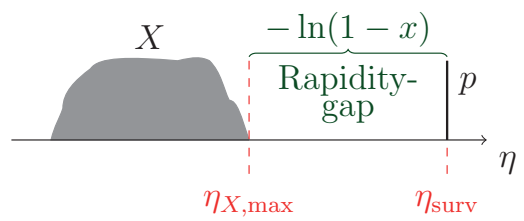

Figure A.4. Kinematics of single diffractive dissociation.

using Equation (124). In a double diffractive process with both hadrons dissociating into two mass systems $M_{X_{1}}, M_{X_{2}}$ one can estimate the rapidity gap with Equation (127),

$$
\Delta \eta=\ln \left(\frac{s_{0} s}{M_{X_{1}}^{2} M_{X_{2}}^{2}}\right),
$$

where $s_{0} \approx 1 \mathrm{GeV}^{2}$.

In central exclusive production the rapidity gap on each side of the central system $X$ is estimated by the fractional momentum loss of the outgoing hadrons given that those can be measured by forward tagging devices,

$$
\Delta \eta_{1,2} \sim \ln \left(\frac{1}{\xi_{1,2}}\right) .
$$

Knowing the kinematics of the outgoing hadrons the mass of the central system can be accurately obtained,

$$
M_{X}=\sqrt{s \xi_{1} \xi_{2}}
$$

\section{Cross Section and Decay Rates}

Transition rates or cross sections are characteristic to scattering processes. The cross section is equivalent to the likelyhood of such a process to happen. In analogy to classical hard-sphere scattering the cross section is the overlap area of the colliding bodies.

A cross section consists of two parts. The amplitude part usually called the matrix element and the phase space part with all kinematical relevant information included. The phase space is directly related to the likelyhood of a process to happen. The smaller the phase space, the more unlikely a process. In case of a process of 2 particles scattering into $n$-particles, the differential cross section can be written as

$$
\begin{aligned}
d \sigma & =\frac{|M(1,2 \rightarrow n)|^{2}}{F} d I \\
& =\frac{|\mathcal{M}(1,2 \rightarrow n)|^{2}}{4\left(\left(p_{1} \cdot p_{2}\right)^{2}-\left(m_{1} m_{2}\right)^{2}\right)} \prod_{i=3}^{n} \frac{d^{4} p_{i}}{(2 \pi)^{3}} \delta\left(p_{i}^{2}-m_{i}^{2}\right)(2 \pi)^{4} \delta^{4}\left(p_{1}+p_{2}-\sum_{i=3}^{n} p_{n}\right),
\end{aligned}
$$


where $M$ is the matrix element of the process, $F$ is the flux factor and $I$ is the phase space term. Suppose one knows already the matrix element $M$ that is the dynamical part calculable for example by perturbative quantum field theories. Using $\delta\left(p^{2}-m^{2}\right)=\delta\left(p^{2}-m^{2}\right) \Theta(E)$ one can simplify the phase space term to

$$
d \sigma=\frac{|\mathcal{M}(1,2 \rightarrow n)|^{2}}{4\left(\left(p_{1} \cdot p_{2}\right)^{2}-\left(m_{1} m_{2}\right)^{2}\right)} \prod_{i=3}^{n} \frac{d^{3} \boldsymbol{p}_{i}}{(2 \pi)^{3} 2 E_{i}}(2 \pi)^{4} \delta^{4}\left(p_{1}+p_{2}-\sum_{i=3}^{n} p_{n}\right) .
$$

The flux factor in the center of mass frame of parallel colliding particles with $\boldsymbol{p}_{\mathbf{1}}=-\boldsymbol{p}_{\mathbf{2}}$ can be written conveniently as,

$$
4\left(\left(p_{1} \cdot p_{2}\right)^{2}-\left(m_{1} m_{2}\right)^{2}\right)=4\left|\boldsymbol{p}_{\mathbf{1}}\right|^{2} s=2 \lambda^{\frac{1}{2}}\left(s, m_{1}^{2}, m_{2}^{2}\right),
$$

where $\lambda(x, y, z)=x^{2}+y^{2}+z^{2}-2 x y-2 x z-2 y z$. In case of an exclusive two-body scattering process, $1+2 \rightarrow 3+4$ in the center of mass frame of two colliding particles, one can simplify the differential cross section formula.

$$
d \sigma=\frac{|\mathcal{M}(1,2 \rightarrow 3,4)|^{2}}{2 \lambda^{\frac{1}{2}}\left(s, m_{1}^{2}, m_{2}^{2}\right)} \frac{d^{3} \boldsymbol{p}_{3}}{(2 \pi)^{3} 2 E_{3}} \frac{d^{3} \boldsymbol{p}_{4}}{(2 \pi)^{3} 2 E_{4}}(2 \pi)^{4} \delta^{4}\left(p_{1}+p_{2}-p_{3}-p_{4}\right)
$$

Now one rewrites the $\delta$-function knowing that $\boldsymbol{p}_{\mathbf{1}}=-\boldsymbol{p}_{\mathbf{2}}$,

$$
\delta^{4}\left(p_{1}+p_{2}-p_{3}-p_{4}\right)=\delta\left(E_{1}+E_{2}-E_{3}-E_{4}\right) \delta^{3}\left(-\boldsymbol{p}_{3}-\boldsymbol{p}_{4}\right)
$$

and integrates over one momentum $\boldsymbol{p}_{\mathbf{4}}$. The Matrix element depends now only on the momentum of one outgoing particle. One would have to integrate over the $\boldsymbol{p}_{3}$ in order to get the cross section. It is convenient to formulate a differential cross section over the solid angle $\Omega$. One can write $d^{3} \boldsymbol{p}_{3}=\left|\boldsymbol{p}_{3}\right|^{2} d\left|\boldsymbol{p}_{3}\right| \sin \vartheta d \vartheta d \phi=\left|\boldsymbol{p}_{3}\right|^{2} d\left|\boldsymbol{p}_{3}\right| d \Omega$. In this case no complete integration is needed and one obtains,

$$
\frac{d \sigma}{d \Omega}=\frac{1}{32 \pi^{2} \lambda^{\frac{1}{2}}\left(s, m_{1}^{2}, m_{2}^{2}\right)} \int_{0}^{\infty}\left|\boldsymbol{p}_{3}\right|^{2} d\left|\boldsymbol{p}_{3}\right| \frac{|\mathcal{M}(1,2 \rightarrow 3,4)|^{2}}{E_{3} E_{4}} \delta\left(E_{1}+E_{2}-E_{3}-E_{4}\right) .
$$

Rewriting the delta function

$$
\delta\left(E_{1}+E_{2}-E_{3}-E_{4}\right)=\delta\left(E_{1}+E_{2}-\sqrt{m_{3}^{2}+\boldsymbol{p}_{3}^{2}}-\sqrt{m_{4}^{2}+\boldsymbol{p}_{3}^{2}}\right)
$$

and assuming high energies, one ends up after the integration over $\left|\boldsymbol{p}_{3}\right|$ with

$$
\frac{d \sigma}{d \Omega}=\frac{|\mathcal{M}(1,2 \rightarrow 3,4)|^{2}}{64 \pi^{2} s},
$$

where $\sqrt{s}=E 1+E 2$ was used.

Particle decays are Poissonian processes, therefore the number of decaying particles follow an exponential function over the time. The decay rate $\Gamma$ is the probability for a particle decay per unit time. The lifetime $\tau$ is the inverse of the decay rate, $\tau=1 / \Gamma$. 
For a number $N$ of one species of particles one has the following expression for the decay rate,

$$
\Gamma=-\frac{N}{d N} d t
$$

The decreasing number of particles necessitates a minus sign. Usually particles decay in several channels, which add to the total decay rate. The total decay rate reads as

$$
\Gamma_{\text {total }}=\sum_{i} \Gamma_{i} .
$$

The ratio between the decay width of one channel to the total decay width is called branching ratio,

$$
B r=\frac{\Gamma_{i}}{\Gamma_{\text {total }}} .
$$

The differential decay rate for particle 1 in its rest frame, decaying into $n-1$ particles, is given by

$$
d \Gamma=\frac{|\mathcal{M}(1,2 \rightarrow n)|^{2}}{2 m_{1}}\left(\prod_{i=2}^{n} \frac{d^{3} \mathbf{p}_{\mathbf{i}}}{(2 \pi)^{3} 2 E_{i}}\right)(2 \pi)^{4} \delta^{4}\left(p_{1}-\sum_{i=2}^{n} p_{i}\right) .
$$

For a general two-body decay into the particles with masses $m_{2}$ and $m_{3}$, Equation (144) simplifies to

$$
d \Gamma=\frac{|\mathbf{p}|}{32 \pi^{2} m_{1}^{2}}|\mathcal{M}|^{2} d \Omega
$$

where

$$
\mathbf{p}=\frac{\lambda^{\frac{1}{2}}\left(m_{1}^{2}, m_{2}^{2}, m_{3}^{3}\right)}{2 m_{1}}
$$

\section{Decay of $\pi^{0}$}

The neutral pion, a light unflavored meson, decays to $\sim 98.8 \%$ into $\gamma \gamma$ and to $\sim 1.2 \%$ into $e^{+} e^{-} \gamma$. In case of the decay into two massless photons, the kinematics can be computed easily. In Figure A.5 the kinematics are illustrated with the pion moving along the $x$-axis and the decay happening within the $x$-y-plane. The 4-momentum vector of the neutral pion is $q_{\pi^{0}}=\left(E_{\pi^{0}}, p_{x_{\pi^{0}}}, 0,0\right)$ and of the photons $q_{\gamma}=\left(E_{\gamma}, p_{x_{\gamma}}, p_{y_{\gamma}}, 0\right)$. In the rest frame of the neutral pion the massless decay products are allways back to back. In the laboratory frame the pion is not at rest therefore one finds an opening angle $\theta_{\gamma \gamma}=\theta+\theta_{r}$ between the products due to the kinematical boost. Here, $\theta$ is the decay angle of one photon with respect to the direction of the moving pion. The decay angle of the other recoiled photon is denoted as $\theta_{r}$. The opening angle is minimal in case the decay is transverse to the direction of the flying pion, $\theta^{*}=90^{\circ}$. The transverse momentum of the photons is half the mass of the pion, $m_{\pi^{0}} / 2$. Because of the transverse decay the transverse momentum stays the same also 


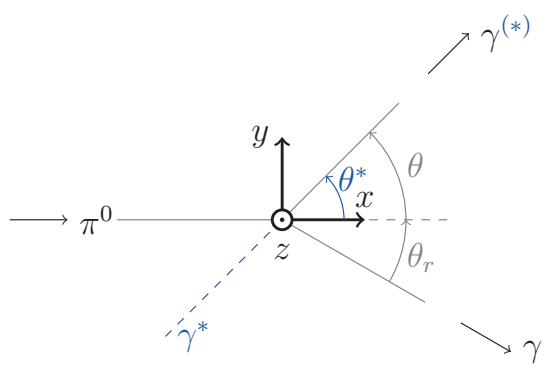

Figure A.5. Kinematics of the neutral pion decay in the laboratory frame (gray) and in the center of mass system, rest frame (blue,*).

in the laboratory frame. The total momentum of one photon follows directly from the pion energy, $E_{\pi^{0}} / 2$. Using trigonometry the minimal opening angle is therefore,

$$
\theta_{\gamma \gamma, \min }=2 \arctan \left(\frac{m_{\pi^{0}}}{E_{\pi^{0}}}\right) .
$$

In the rest frame the decay of the neutral pion is isotropic which gives a flat distribution of the photons with respect to $\cos \theta^{*}$,

$$
\frac{d N}{d \cos \theta^{*}}=\frac{1}{2}
$$

The energy spectrum of the photons in the laboratory frame using above equation is,

$$
\frac{d N}{d E_{\gamma}}=\frac{d \cos \theta^{*}}{2 d E_{\gamma}} .
$$

In the pion rest frame the photon energy $E^{*}$ is half of the pion mass. Also the photon energy is equal to its momentum. The 4-momentum vector can be written as $q_{\gamma}^{*}=m_{\pi^{0}} / 2\left(1, \cos \theta^{*}, \sin \theta^{*}, 0\right)$. The energy of the photon $E_{\gamma}$ in the laboratory frame can be obtained by using the Lorentz transformation,

$$
\left(\begin{array}{c}
E \\
p_{\|}
\end{array}\right)=\left(\begin{array}{cc}
\gamma & \gamma \beta \\
\gamma \beta & \gamma
\end{array}\right)\left(\begin{array}{c}
E^{*} \\
p_{\|}^{*}
\end{array}\right)
$$

where $p_{\|}$is the momentum parallel to the moving direction of the frame. Thus,

$$
E_{\gamma}=m_{\pi^{0}} / 2\left(\gamma+\gamma \beta \cos \theta^{*}\right) .
$$

Putting this into Equation (149) one gets,

$$
\frac{d N}{d E_{\gamma}}=\frac{1}{\gamma \beta m_{\pi^{0}}}=\frac{1}{\left|\boldsymbol{p}_{\pi^{0}}\right|},
$$

which is a flat distribution between $\left(E_{\pi^{0}}-\left|\boldsymbol{p}_{\pi^{0}}\right|\right)$ and $\left(E_{\pi^{0}}+\left|\boldsymbol{p}_{\pi^{0}}\right|\right)$. 



\section{APPENDIX B \\ Event Displays}

1. Typical Exclusive Photon Pairs
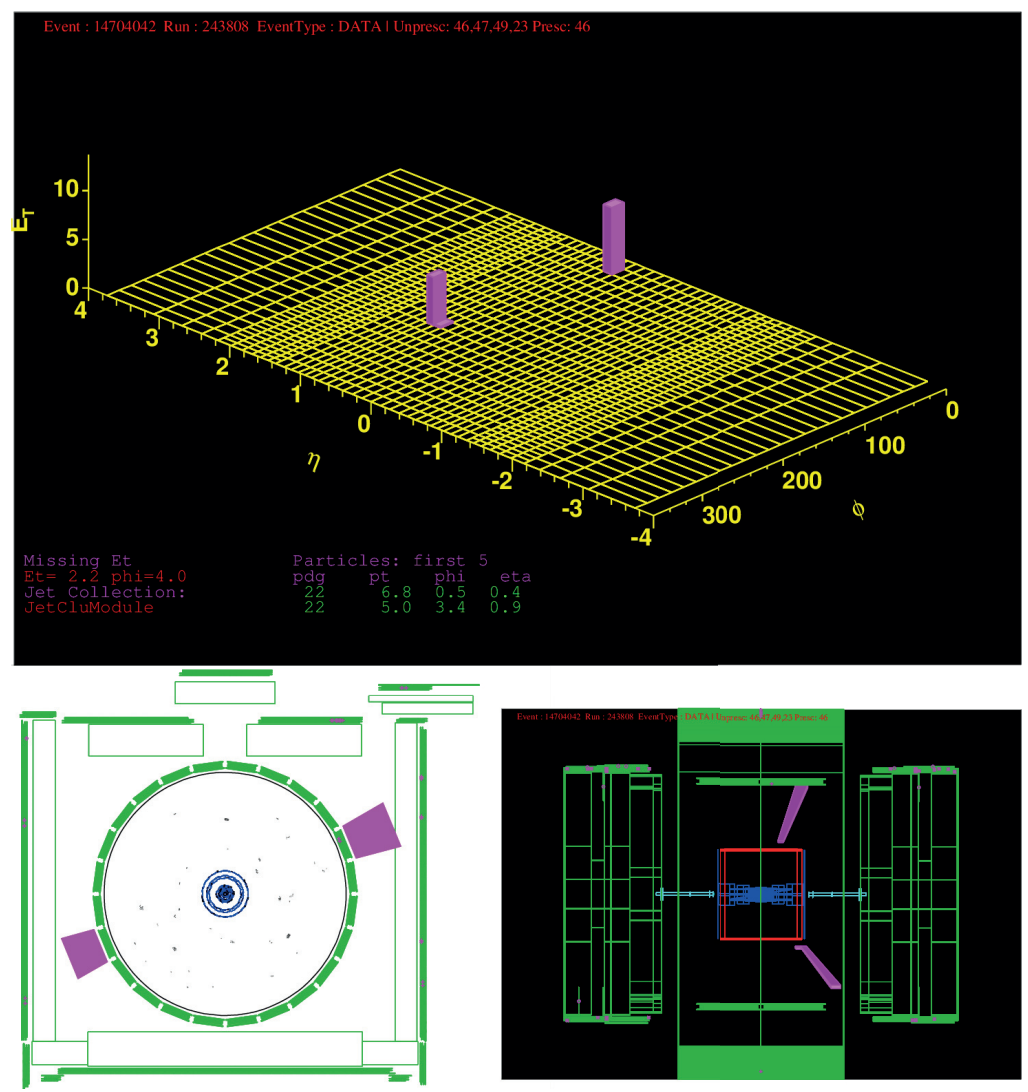

Figure B.1. Exclusive photon pair event from candidate sample with high invariant mass. 

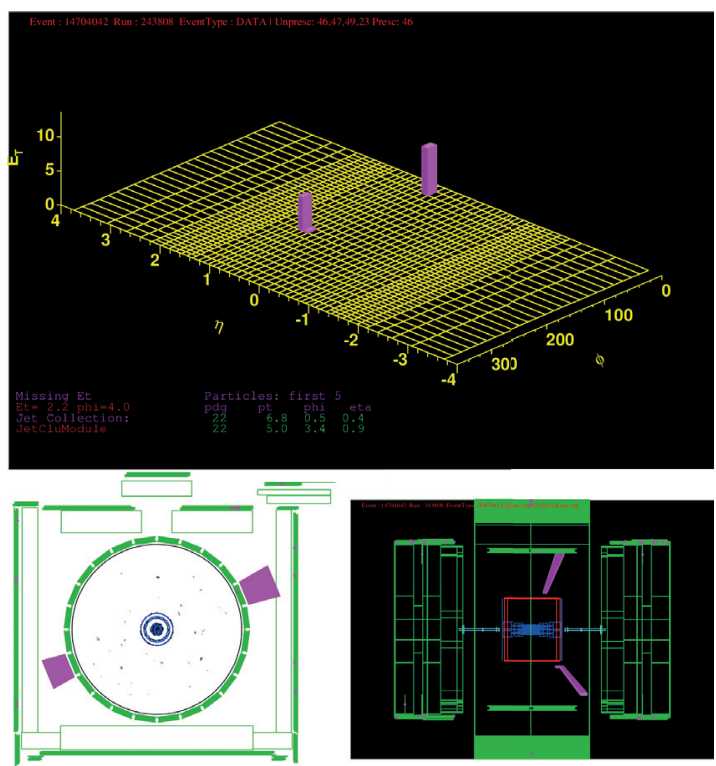

Figure B.2. Exclusive photon pair event from candidate sample with low invariant mass.
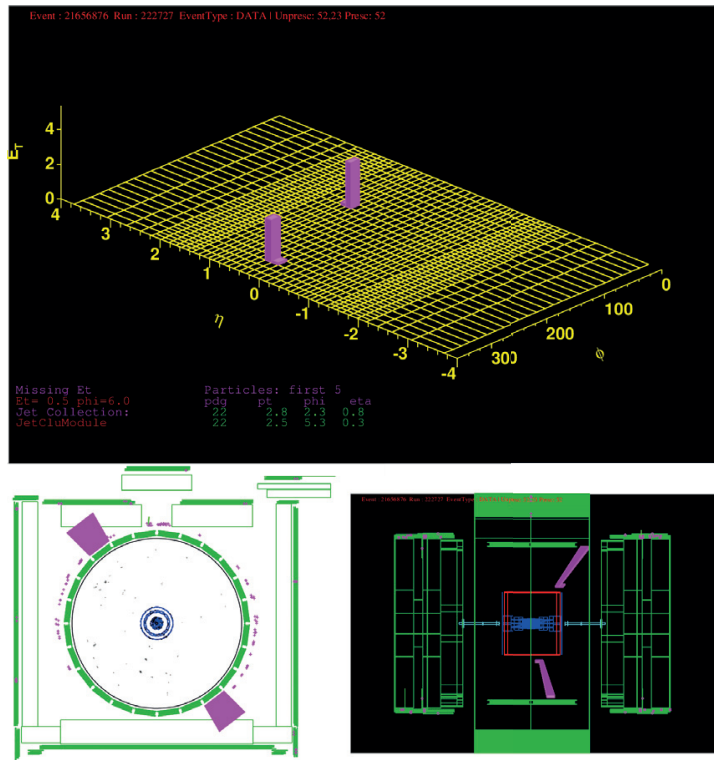

FiguRE B.3. Exclusive photon pair event from candidate sample with low invariant mass. 


\section{Typical Exclusive Electron-Positron Pairs}
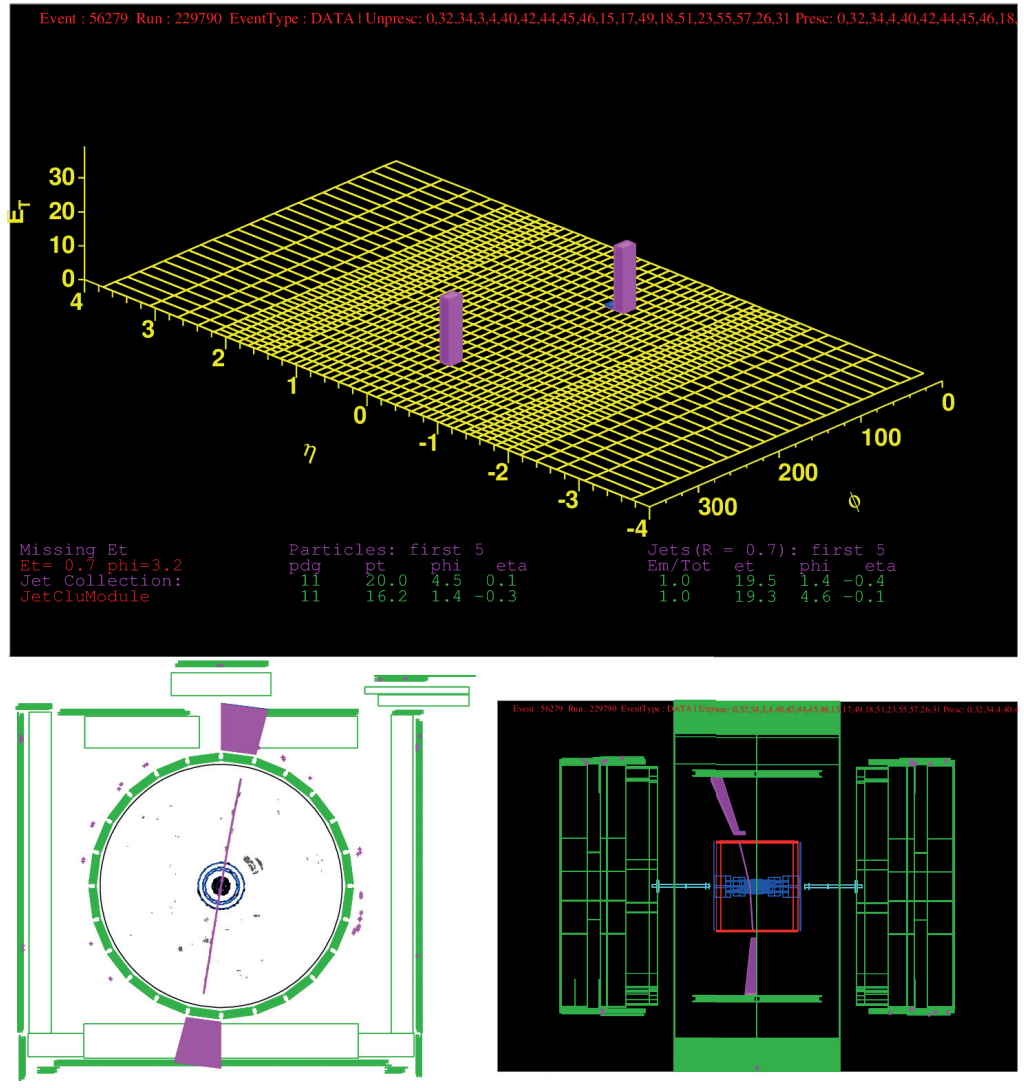

Figure B.4. Exclusive electron-positron pair event from candidate sample with the highest invariant mass. 

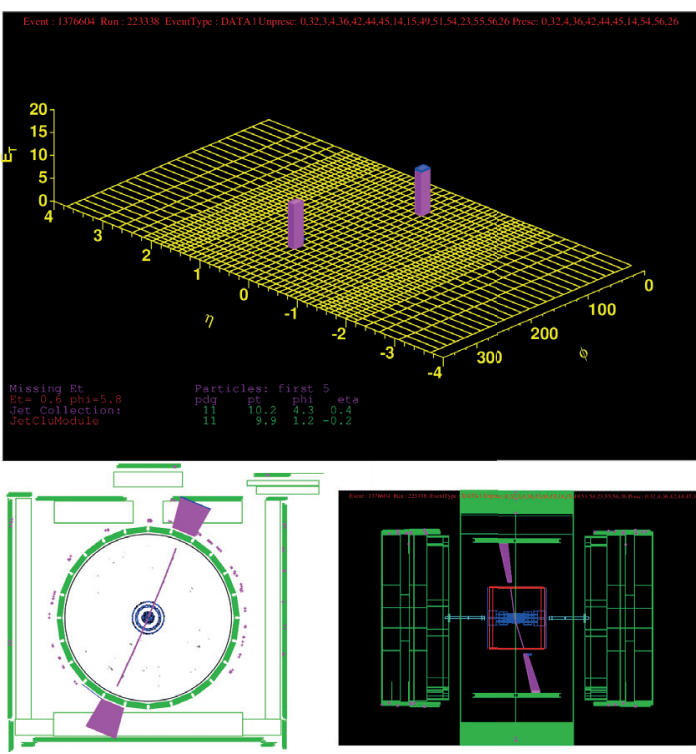

FIGURE B.5. Exclusive electron-positron pair event from candidate sample with high invariant mass.
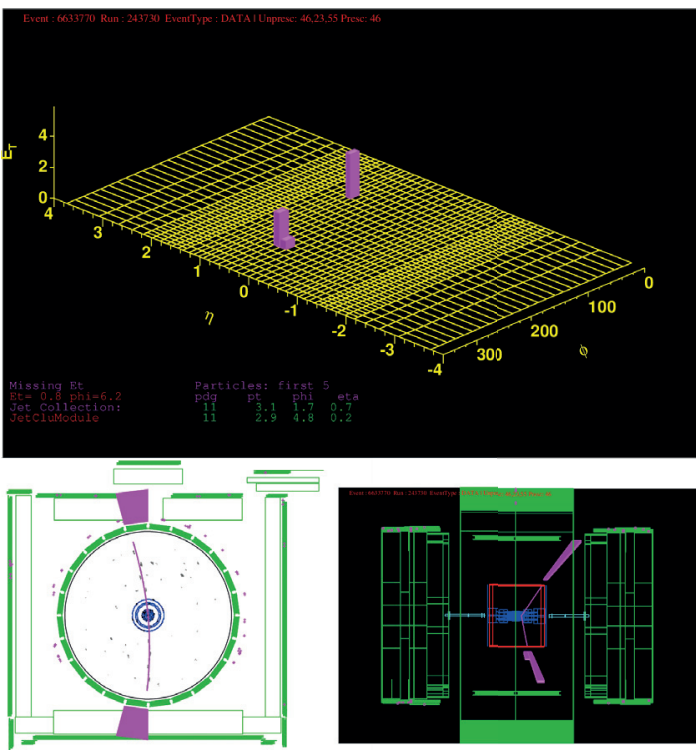

FiguRE B.6. Exclusive electron-positron pair event from candidate sample with low invariant mass. 


\section{Bibliography}

[1] V.A. Khoze, A.D. Martin, M.G. Ryskin and W.J. Stirling, Diffractive $\gamma \gamma$ production at hadron colliders, Eur. Phys. J. C 38, 475 (2005).

[2] L.A. Harland-Lang, V.A. Khoze, M.G. Ryskin, and W.J. Stirling, Standard candle central exclusive processes at the Tevatron and LHC, Eur. Phys. J. C 69, 179 (2010).

[3] L.A. Harland-Lang, V.A. Khoze, M.G. Ryskin and W.J. Stirling, The Phenomenology of Central Exclusive Production at Hadron Colliders, Eur. Phys. J. C 72, 2110 (2012).

[4] M.G. Albrow, T.D. Coughlin and J.R. Forshaw, Central Exclusive Particle Production at High Energy Hadron Colliders, Prog. Part. Nucl. Phys. 65, 149 (2010).

[5] M.G. Albrow et al., A Search for the Higgs boson using very forward tracking detectors with $C D F$, FERMILAB-LOI-2005-01, arXiv:hep-ex/0511057 (2001).

[6] L.A. Harland-Lang, V.A. Khoze, M.G. Ryskin and W.J. Stirling, Central exclusive production as a probe of the gluonic component of the eta' and eta mesons, arXiv:1302.2004 [hep-ph].

[7] V.A. Khoze, A.D. Martin and M.G. Ryskin, The Rapidity gap Higgs signal at LHC, Phys. Lett. B 401, 330 (1997).

[8] V.A. Khoze, A.D. Martin, and M.G. Ryskin, Can the Higgs be seen in rapidity gap events at the Tevatron or the LHC?, Eur. Phys. J. C 14, 525 (2000).

[9] V.A. Khoze, A.D. Martin, and M.G. Ryskin, Prospects for new physics observations in diffractive processes at the LHC and Tevatron, Eur. Phys. J. C 23, 311 (2002), and references therein.

[10] A. Abulencia et al. (CDF Collaboration), Observation of Exclusive Electron-Positron Production in Hadron-Hadron Collisions, Phys. Rev. Lett. 98, 112001 (2007).

[11] T. Aaltonen et al. (CDF Collaboration), Search for exclusive $\gamma \gamma$ production in hadron-hadron collisions, Phys. Rev. Lett. 99, 242002 (2007).

[12] M.E. Peskin and D.V. Schroeder, An Introduction to quantum field theory, Reading, USA: Addison-Wesley (1995).

[13] K. Nakamura et al. (Particle Data Group Collaboration), Review of particle physics, J. Phys. G G 37, 075021 (2010).

[14] T. Aaltonen et al. (CDF Collaboration), Precise measurement of the $W$-boson mass with the CDF II detector, Phys. Rev. Lett. 108, 151803 (2012).

[15] G. Aad et al. (ATLAS Collaboration), Observation of a new particle in the search for the Standard Model Higgs boson with the ATLAS detector at the LHC, Phys. Lett. B 716, 1 (2012).

[16] S. Chatrchyan et al. (CMS Collaboration), Observation of a new boson at a mass of $125 \mathrm{GeV}$ with the CMS experiment at the LHC, Phys. Lett. B 716, 30 (2012).

[17] A.B. Kaidalov, V.A. Khoze, A.D. Martin and M.G. Ryskin, Central exclusive diffractive production as a spin-parity analyser: From Hadrons to Higgs, Eur. Phys. J. C 31, 387 (2003).

[18] S. Bethke, The 2009 World Average of alpha(s), Eur. Phys. J. C 64 (2009) 689.

[19] J.E. Brücken, Cascade Decays of Neutralinos and Charginos at Benchmark Points, HIP Internal Report, HIP-2003-03 (2003).

[20] Yu.A. Gol'fand and E.P. Likhtman, Extension of the Algebra of Poincare Group Generators and Violation of $p$ Invariance, JETP Lett. 13, 323 (1971).

[21] V.P. Akulov and D.V. Volkov, Is the Neutrino a Goldstone Particle?, Phys. Lett. B 46, 109 (1973). 
[22] J. Wess and B. Zumino, Supergauge Transformations in Four-Dimensions, Nucl. Phys. B70, 39 (1974).

[23] C. Gattringer and C.B. Lang, Quantum chromodynamics on the lattice, Lect. Notes Phys. 788, $1(2010)$.

[24] R.K. Ellis, W.J. Stirling and B.R. Webber, QCD and collider physics, Camb. Monogr. Part. Phys. Nucl. Phys. Cosmol. 8, 1 (1996).

[25] J.D. Bjorken, Asymptotic Sum Rules at Infinite Momentum, Phys. Rev. 179, 1547 (1969).

[26] A.D. Martin, W.J. Stirling, R.S. Thorne and G. Watt, Parton distributions for the LHC, Eur. Phys. J. C 63, 189 (2009).

[27] V.N. Gribov, L,N. Lipatov, Deep inelastic e p scattering in perturbation theory, Sov. J. Nucl. Phys. 15, 438 (1972).

[28] G. Altarelli and G. Parisi, Asymptotic Freedom in Parton Language, Nucl. Phys. B126, 298 (1977).

[29] Yu.L. Dokshitzer, Calculation of the Structure Functions for Deep Inelastic Scattering and $e^{+} e^{-}$ Annihilation by Perturbation Theory in Quantum Chromodynamics, Sov. Phys. JETP 46, 641 (1977).

[30] G. Antchev et al., First measurement of the total proton-proton cross section at the LHC energy of $\sqrt{s}=7 \mathrm{TeV}$, Europhys. Lett. 96, 21002 (2011).

[31] M. Froissart, Asymptotic behavior and subtractions in the Mandelstam representation, Phys. Rev. 123, 1053 (1961).

[32] L. Lukaszuk and A. Martin, Absolute upper bounds for pi pi scattering, Nuovo Cim. A 52, 122 (1967).

[33] R. Fiore, L.L. Jenkovszky, R. Orava, E. Predazzi, A. Prokudin and O. Selyugin, Forward Physics at the LHC: Elastic Scattering, Int. J. Mod. Phys. A 24, 2551 (2009).

[34] I. Bautista and J.D. de Deus, The black disk and the dip in the differential elastic cross section at asymptotic energy, Phys. Lett. B 718, 1571 (2013).

[35] M.L. Good and W.D. Walker, Diffraction disssociation of beam particles, Phys. Rev. 120, 1857 (1960).

[36] H.I. Miettinen and J. Pumplin, Diffraction Scattering and the Parton Structure of Hadrons, Phys. Rev. D 18, 1696 (1978).

[37] S. Sapeta, Diffractive production at high energies in the Miettinen-Pumplin model, Phys. Lett. B 597, 352 (2004).

[38] R.P. Feynman, Photon-hadron interactions, Reading 1972, 282p.

[39] V.N. Gribov, Space-time description of hadron interactions at high-energies, Lecture material of the VIII Winter Petersburg NPI School, arXiv:hep-ph/0006158 (English translation) (1973).

[40] J.D. Bjorken, Hadron Final States in Deep Inelastic Processes, Lect. Notes Phys. 56, 93 (1976).

[41] E. Gotsman, E. Levin and U. Maor, N=4 SYM and QCD motivated approach to soft interactions at high energies, Eur. Phys. J. C 71, 1553 (2011).

[42] S. Donnachie, G. Dosch, P.V. Landshoff, and O. Nachtmann, Pomeron Physics and QCD, (Cambridge University Press, Cambridge, U.K., 2002).

[43] A.B. Kaidalov, Diffractive Production Mechanisms, Phys. Rept. 50, 157 (1979).

[44] S.N. Ganguli and D.P. Roy, Regge phenomenology and inclusive reactions, Phys. Rept. 67, 201 (1980).

[45] J.R. Forshaw and D.A. Ross, Quantum Chromodynamics and the Pomeron, (Cambridge University Press, Cambridge, U.K., 1997).

[46] A. Donnachie and P.V. Landshoff, Total cross-sections, Phys. Lett. B 296, 227 (1992).

[47] A.H. Mueller, O(2,1) Analysis Of Single Particle Spectra At High-energy, Phys. Rev. D 2, 2963 (1970).

[48] V.A. Khoze, A.D. Martin and M.G. Ryskin, Soft diffraction and the elastic slope at Tevatron and LHC energies: A MultiPomeron approach, Eur. Phys. J. C 18, 167 (2000). 
[49] A.B. Kaidalov, V.A. Khoze, Y.F. Pirogov and N.L. Ter-Isaakyan, On determination of the triple pomeron coupling from the ISR data, Phys. Lett. B 45, 493 (1973).

[50] A.B. Kaidalov and K.A. Ter-Martirosyan, The pomeron-particle total cross-section and diffractive production of showers at very high energies, Nucl. Phys. B 75, 471 (1974).

[51] Y.I. Azimov, V.A. Khoze, E.M. Levin and M.G. Ryskin, Estimates of the Cross-Sections for Double-Reggeon Processes, Sov. J. Nucl. Phys. 21, 215 (1975) [Yad. Fiz. 21, 413 (1975)].

[52] E.G.S. Luna, V.A. Khoze, A.D. Martin and M.G. Ryskin, Diffractive dissociation re-visited for predictions at the LHC, Eur. Phys. J. C 59, 1 (2009).

[53] F.E. Low, A Model of the Bare Pomeron, Phys. Rev. D 12, 163 (1975).

[54] S. Nussinov, Colored Quark Version of Some Hadronic Puzzles, Phys. Rev. Lett. 34, 1286 (1975).

[55] J.F. Gunion and D.E. Soper, Quark Counting and Hadron Size Effects for Total Cross-Sections, Phys. Rev. D 15, 2617 (1977).

[56] E.A. Kuraev, L.N. Lipatov and V.S. Fadin, Multi - Reggeon Processes in the Yang-Mills Theory, Sov. Phys. JETP 44, 443 (1976) [Zh. Eksp. Teor. Fiz. 71, 840 (1976)].

[57] E.A. Kuraev, L.N. Lipatov and V.S. Fadin, The Pomeranchuk Singularity in Nonabelian Gauge Theories, Sov. Phys. JETP 45, 199 (1977) [Zh. Eksp. Teor. Fiz. 72, 377 (1977)].

[58] L.N. Lipatov, Reggeization of the Vector Meson and the Vacuum Singularity in Nonabelian Gauge Theories, Sov. J. Nucl. Phys. 23, 338 (1976) [Yad. Fiz. 23, 642 (1976)].

[59] I.I. Balitsky and L.N. Lipatov, The Pomeranchuk Singularity in Quantum Chromodynamics, Sov. J. Nucl. Phys. 28, 822 (1978) [Yad. Fiz. 28, 1597 (1978)].

[60] B.L. Ioffe, (ed.), V.S. Fadin, (ed.) and L.N. Lipatov, (ed.), Quantum chromodynamics: Perturbative and nonperturbative aspects, (Cambridge University Press, Cambridge, U.K., 2010).

[61] A.D. Martin, Proton structure, Partons, QCD, DGLAP and beyond, Acta Phys. Polon. B 39, 2025 (2008).

[62] V.A. Khoze, A.D. Martin and M.G. Ryskin, Double diffractive processes in high resolution missing mass experiments at the Tevatron, Eur. Phys. J. C 19, 477 (2001).

[63] V.A. Khoze, A.D. Martin and M.G. Ryskin, Double diffractive processes in high resolution missing mass experiments at the Tevatron, Erratum: Eur. Phys. J. C 20, 599 (2001).

[64] A.B. Kaidalov, V.A. Khoze, A.D. Martin and M.G. Ryskin, Extending the study of the Higgs sector at the LHC by proton tagging, Eur. Phys. J. C 33, 261 (2004).

[65] A.D. Martin and M.G. Ryskin, Unintegrated generalized parton distributions, Phys. Rev. D 64, 094017 (2001).

[66] A.D. Martin, C. Nockles, M.G. Ryskin, A.G. Shuvaev and T. Teubner, Generalised parton distributions at small $x$, Eur. Phys. J. C 63, 57 (2009).

[67] M.A. Kimber, A.D. Martin and M.G. Ryskin, Unintegrated parton distributions, Phys. Rev. D 63, 114027 (2001).

[68] V.A. Khoze, A.D. Martin and M.G. Ryskin, Physics with tagged forward protons at the LHC, Eur. Phys. J. C 24, 581 (2002).

[69] M.G. Ryskin, A.D. Martin and V.A. Khoze, Soft processes at the LHC. II. Soft-hard factorization breaking and gap survival, Eur. Phys. J. C 60, 265 (2009).

[70] L.A. Harland-Lang, V.A. Khoze, M.G. Ryskin and W.J. Stirling, Central exclusive meson pair production in the perturbative regime at hadron colliders, Eur. Phys. J. C 71, 1714 (2011).

[71] V.A. Khoze, A.D. Martin, and M.G. Ryskin, Diffractive Higgs production: Myths and reality, Eur. Phys. J. C 26, 229 (2002) and references therein.

[72] A. Bialas and P.V. Landshoff, Higgs production in $p$ p collisions by double pomeron exchange, Phys. Lett. B 256, 540 (1991).

[73] A. Schafer, O. Nachtmann and R. Schopf, Production of Higgs particles in diffractive hadron hadron collisions, Phys. Lett. B 249, 331 (1990).

[74] T.D. Coughlin and J.R. Forshaw, Central Exclusive Production in QCD, J. High Energy Phys. 01 (2010) 121. 
[75] M.G. Albrow et al. (FP420 R and D Collaboration), The FP420 R\&D Project: Higgs and New Physics with forward protons at the LHC, JINST 4, T10001 (2009).

[76] A.J. Bell et al., Physics and Beam Monitoring with Forward Shower Counters (FSC) in CMS, FERMILAB-PUB-10-718-CMS (2010).

[77] L.A. Harland-Lang, V.A. Khoze, M.G. Ryskin and W.J. Stirling, Latest Results in Central Exclusive Production: A Summary, arXiv:1301.2552 [hep-ph].

[78] B.E. Cox, F.K. Loebinger and A.D. Pilkington, Detecting Higgs bosons in the b $\bar{b}$ decay channel using forward proton tagging at the LHC, JHEP 0710, 090 (2007).

[79] V.M. Budnev, I.F. Ginzburg, G.V. Meledin and V.G. Serbo, The Two photon particle production mechanism. Physical problems. Applications. Equivalent photon approximation, Phys. Rept. 15, 181 (1975).

[80] V.A. Khoze, private communications.

[81] V.A. Khoze, A.D. Martin, R. Orava and M.G. Ryskin, Luminosity monitors at the LHC, Eur. Phys. J. C 19, 313 (2001).

[82] T. Aaltonen et al. (CDF Collaboration), Observation of exclusive charmonium production and $\gamma \gamma \rightarrow \mu^{+} \mu^{-}$in $p \bar{p}$ collisions at $\sqrt{s}=1.96 \mathrm{TeV}$, Phys. Rev. Lett. 102, 242001 (2009).

[83] R. Aaij et al. (LHCb Collaboration), Exclusive $J / \psi$ and $\psi(2 S)$ production in pp collisions at $\sqrt{s}=7 \mathrm{TeV}$, arXiv:1301.7084 [hep-ex].

[84] V.A. Khoze, A.D. Martin and M.G. Ryskin, Photon exchange processes at hadron colliders as a probe of the dynamics of diffraction, Eur. Phys. J. C 24, 459 (2002).

[85] R. Blair et al. (CDF Collaboration), The CDF-II detector: Technical design report, FERMILABDESIGN-1996-01.

[86] D. Acosta et al. (CDF Collaboration), Measurement of the $J / \psi$ meson and $b$-hadron production cross sections in p $\bar{p}$ collisions at $\sqrt{s}=1960$ GeV, Phys. Rev. D 71, 032001 (2005) and references therein.

[87] D. Amidei et al. (CDF Collaboration), The Silicon Vertex Detector of the Collider Detector at Fermilab, Nucl. Instrum. Methods 350, 73 (1994).

[88] F. Abe et al. (CDF Collaboration), Evidence for top quark production in $\bar{p} p$ collisions at $\sqrt{s}=1.8$ TeV, Phys. Rev. D 50, 2966 (1994).

[89] A. Abulencia, et al. (CDF Collaboration), Measurements of inclusive $W$ and $Z$ cross sections in $p \bar{p}$ collisions at $\sqrt{s}=1.96$ TeV, J. Phys. G Nucl. Part. Phys. 34, 2457 (2007).

[90] T. Aaltonen et al. (CDF Collaboration), Diffractive $W$ and $Z$ Production at the Fermilab Tevatron, Phys. Rev. D 82, 112004 (2010).

[91] S. Behari, private communications.

[92] A. Affolder et al. (CDF Collaboration), CDF central outer tracker, Nucl. Instrum. Methods Phys. Res. Sect. A 526, 249 (2004).

[93] L. Balka et al. (CDF Collaboration), The CDF Central Electromagnetic Calorimeter, Nucl. Instrum. Methods A267, 272 (1988).

[94] S. Bertolucci et al. (CDF Collaboration), The CDF Central and Endwall Hadron Calorimeter, Nucl. Instrum. Methods A267, 301 (1988).

[95] M. Albrow et al. (CDF Collaboration), The CDF plug upgrade electromagnetic calorimeter: Test beam results, Nucl. Instrum. Methods, A480, 524 (2002).

[96] S. Lami (CDF Collaboration), The CDF MiniPlug calorimeters, Nucl. Instrum. Meth. A 496, 333 (2003).

[97] D. Acosta et al. (CDF Collaboration), The CDF Cherenkov luminosity monitor, Nucl. Instrum. Meth. A461, 540 (2001).

[98] D. Acosta et al., The performance of the CDF luminosity monitor, Nucl. Instrum. Methods A494, 57 (2002).

[99] L. Baksay et al., Evidence for Double Pomeron Exchange at the CERN ISR, Phys. Lett. B 61, 89 (1976). 
[100] T. Akesson et al. (Axial Field Spectrometer Collaboration), A Search For Glueballs And A Study Of Double Pomeron Exchange At The Cern Intersecting Storage Rings, Nucl. Phys. B 264, 154 (1986).

[101] P. Minkowski and W. Ochs, Identification of the glueballs and the scalar meson nonet of lowest mass, Eur. Phys. J. C 9, 283 (1999).

[102] A. Breakstone et al. (Ames-Bologna-CERN-Dortmund-Heidelberg-Warsaw Collaboration), Production of the $f^{0}$ Meson in the Double Pomeron Exchange Reaction $p p \rightarrow p p \pi^{+} \pi^{-}$at $\sqrt{s}=62 \mathrm{GeV}$, Z. Phys. C 31, 185 (1986).

[103] A. Breakstone et al. (Ames-Bologna-CERN-Dortmund-Heidelberg-Warsaw Collaboration), The Reaction Pomeron-Pomeron $\rightarrow \pi^{+} \pi^{-}$and an unusual production mechanism for the $f_{2}(1270)$, Z. Phys. C 48, 569 (1990).

[104] A. Brandt et al. (UA8 Collaboration), A Study of inclusive double pomeron exchange in $p \bar{p} \rightarrow p X \bar{p}$ at $\sqrt{s}=630-G e V$, Eur. Phys. J. C 25, 361 (2002).

[105] D. Joyce, A. Kernan, M. Lindgren, D. Smith, S.J. Wimpenny, M.G. Albrow, B.H. Denby aTaken from $[\mathbf{1 9}]$.nd G. Grayer, Double pomeron exchange studies in $p$ anti-p interactions at 0.63-TeV, Phys. Rev. D 48, 1943 (1993).

[106] T. Aaltonen et al. (CDF Collaboration), Observation of Exclusive Dijet Production at the Fermilab Tevatron $\bar{p} p$ Collider, Phys. Rev. D 77, 052004 (2008).

[107] J. Monk and A. Pilkington, ExHuME: A Monte Carlo event generator for exclusive diffraction, Comput. Phys. Commun. 175, 232 (2006).

[108] T. Aaltonen et al. (CDF Collaboration), Search for exclusive $Z$ boson production and observation of high mass $p \bar{p} \rightarrow \gamma \gamma \rightarrow p+\ell \ell+\bar{p}$ events in $p \bar{p}$ collisions at $\sqrt{s}=1.96$ TeV, Phys. Rev. Lett. 102, 222002 (2009).

[109] R. Brun and F. Rademakers, ROOT: An object oriented data analysis framework, Nucl. Instrum. Meth. A 389, 81 (1997).

[110] A. Baranovski et al., The SAM-GRID project: Architecture and plan, Nucl. Instrum. Meth. A 502, 423 (2003).

[111] W. Budgett et al., The Good Run List, CDF Internal Note 5613 (2001).

[112] W. Orejudos, COT Calibration Software, CDF Internal Note 5656 (2003).

[113] B. Heinemann, Offline Calibration of the Calorimeter, CDF Internal Note 6406 (2003).

[114] T. Sjostrand, S. Mrenna and P.Z. Skands, PYTHIA 6.4 Physics and Manual, JHEP 0605, 026 (2006).

[115] T. Gleisberg, S. Hoeche, F. Krauss, M. Schonherr, S. Schumann, F. Siegert and J. Winter, Event generation with SHERPA 1.1, JHEP 0902, 007 (2009).

[116] H.L. Lai et al. (CTEQ Collaboration), Global QCD analysis of parton structure of the nucleon: CTEQ5 parton distributions, Eur. Phys. J. C 12, 375 (2000).

[117] L.A. Harland-Lang, V.A. Khoze, M.G. Ryskin and W.J. Stirling, Central exclusive chi(c) meson production at the Tevatron revisited, Eur. Phys. J. C 65, 433 (2010).

[118] The superCHIC code and documentation are available at http://projects.hepforge.org/superchic/.

[119] A.D. Martin, R.G. Roberts, W.J. Stirling and R.S. Thorne, Parton distributions and the LHC: $W$ and $Z$ production, Eur. Phys. J. C 14, 133 (2000).

[120] S.P. Baranov, O. Duenger, H. Shooshtari and J.A.M. Vermaseren, LPAIR: A generator for lepton pair production, Proceedings of Physics at HERA, vol. 3, 1478-1482 (1991).

[121] J.A.M. Vermaseren, Two Photon Processes at Very High-Energies, Nucl. Phys. B 229, 347 (1983).

[122] R. Ciesielski and K. Goulianos, MBR Monte Carlo Simulation in PYTHIA8, arXiv:1205.1446 [hep-ph].

[123] E. Gerchtein and M. Paulini, CDF detector simulation framework and performance, eConf C 0303241, TUMT005 (2003). 
[124] L. Garren, STDheP: A common output format for Monte Carlo events, http://cepa.fnal.gov/psm/stdhep/.

[125] GEANT, detector simulation and simulation tool, CERN Program Library Long Writeup W5013 (1993).

[126] G. Grindhammer, M. Rudowicz and S. Peters, The Fast Simulation Of Electromagnetic And Hadronic Showers, Nucl. Instrum. Meth. A 290, 469 (1990).

[127] R. Veenhof, GARFIELD, recent developments, Nucl. Instrum. Meth. A 419, 726 (1998).

[128] G.E.P. Box, Sampling and Bayes' inference in scientific modelling and robustness [with discussion], J. R. Statist. Soc. A 143, 383 (1980).

[129] L. Demortier, P Values: What They Are and How to Use Them, CDF Internal Note 8662 (2007).

[130] A. Bhatti et al. (CDF Collaboration), Determination of the jet energy scale at the collider detector at Fermilab, Nucl. Instrum. Meth. A 566, 375 (2006).

[131] A. Wyatt, A Search for Exclusive $J / \psi+\gamma$ Production, CDF Internal Note 6646 (2003).

[132] G.R. Farrar and D.R. Jackson, The Pion Form-Factor, Phys. Rev. Lett. 43, 246 (1979).

[133] S. Striganov, private communications.

[134] W.R. Nelson, H. Hirayama and D.W.O. Rogers, The Egs4 Code System, SLAC-0265 (1985).

[135] M.G. Ryskin, A.D. Martin and V.A. Khoze, Proton Opacity in the Light of LHC Diffractive Data, Eur. Phys. J. C 72, 1937 (2012).

[136] S. Chatrchyan et al. (CMS Collaboration), Search for exclusive or semi-exclusive photon pair production and observation of exclusive and semi-exclusive electron pair production in pp collisions at $\sqrt{s}=7 \mathrm{TeV}$, JHEP 1211, 080 (2012).

[137] V.A. Khoze, M.G. Albrow, private communications. 